\title{
WestVirginiaUniversity
}

THE RESEARCH REPOSITORY @ WVU

Graduate Theses, Dissertations, and Problem Reports

2020

\section{Structural Characterization of Emerging Synthetic Drugs}

Jay Tyler Davidson

West Virgina University, jtdavidson@mix.wvu.edu

Follow this and additional works at: https://researchrepository.wvu.edu/etd

Part of the Analytical Chemistry Commons

\section{Recommended Citation}

Davidson, Jay Tyler, "Structural Characterization of Emerging Synthetic Drugs" (2020). Graduate Theses, Dissertations, and Problem Reports. 7584.

https://researchrepository.wvu.edu/etd/7584

This Dissertation is protected by copyright and/or related rights. It has been brought to you by the The Research Repository @ WVU with permission from the rights-holder(s). You are free to use this Dissertation in any way that is permitted by the copyright and related rights legislation that applies to your use. For other uses you must obtain permission from the rights-holder(s) directly, unless additional rights are indicated by a Creative Commons license in the record and/ or on the work itself. This Dissertation has been accepted for inclusion in WVU Graduate Theses, Dissertations, and Problem Reports collection by an authorized administrator of The Research Repository @ WVU.

For more information, please contact researchrepository@mail.wvu.edu. 


\title{
Structural Characterization of Emerging Synthetic Drugs
}

\author{
J. Tyler Davidson
}

Dissertation submitted to the Eberly College of Arts and Sciences at West Virginia University in partial fulfillment of the requirements for the degree of

Doctor of Philosophy in Forensic Science

Glen P. Jackson, Ph.D., Chair

Luis E. Arroyo, Ph.D.

Tatiana Trejos, Ph.D.

Stephen J. Valentine, Ph.D.

Department of Forensic and Investigative Science

Morgantown, West Virginia

2020

Keywords: Structural characterization, Emerging synthetic drugs, Multistage mass spectrometry $\left(\mathrm{MS}^{\mathrm{n}}\right)$, High-resolution mass spectrometry (HRMS), Synthetic cathinones, Fentanyl analogs Copyright 2020 J. Tyler Davidson 


\section{Abstract \\ Structural Characterization of Emerging Synthetic Drugs}

J. Tyler Davidson

The identification of well-characterized seized drugs is performed thousands of times a day in the United States; however, the expanding use of emerging synthetic drugs is creating a growing problem for both toxicological and seized drug analyses. Two of the most rapidly growing areas of emerging synthetic drugs are synthetic cathinones and fentanyl-related compounds (FRCs). In this work we demonstrate the combination of multi-stage mass spectrometry $\left(\mathrm{MS}^{\mathrm{n}}\right)$, accurate mass measurements with high-resolution mass spectrometry (HRMS), and isotopic labeling for the structural characterization of synthetic cathinones and fentanyl analogs. The deliverables of this research include the identification of conserved fragmentation pathways for synthetic cathinones and fentanyl analogs, proposed mechanisms for the formation of characteristic ions through both protonated tandem mass spectrometry (MS/MS) and electron ionization mass spectrometry (EIMS), and a discussion about how to apply the broadened understanding of the fragmentation behavior to the identification of novel synthetic cathinones and fentanyl analogs.

The first major finding about the fragmentation behavior of synthetic cathinones is that the tropylium ion $(\mathrm{m} / \mathrm{z}$ 91), or substituted derivative thereof, forms through different oxygencontaining intermediates that do not contain a formal $\mathrm{C}=\mathrm{O}$ bond but instead contain a phthalanelike core structure. The phthalane-like intermediates were elucidated through gas-phase ion spectroscopy measurements and density functional theory (DFT) calculations. Likewise, the use of stable isotope labeling revealed the unprecedented finding that, during collision-induced dissociation (CID) of $\alpha$-pyrrolidinophenone synthetic cathinones, the $\alpha$-carbon is retained almost exclusively in the tropylium ion and the carbonyl carbon is not retained in the tropylium ion. Isotope labeling also identified competitive pathways for the loss of $\mathrm{CO}$ and ethylene $\left(\mathrm{C}_{2} \mathrm{H}_{4}\right)$ from a primary intermediate ion, which provides support for the direct loss of $\mathrm{CO}$ from the alkyl side chain.

A second major finding was the identification of characteristic protonated MS/MS fragmentation pathways and proposed mechanistic origins for both protonated MS/MS and EI-MS fragmentation for $\alpha$-pyrrolidinophenone and $N$-alkylated synthetic cathinones. For MS/MS spectra of protonated $\alpha$-pyrrolidinophenone synthetic cathinones the dominant fragmentation pathways are through 4-center hydrogen rearrangements to produce pyrrolidine ring cleavage, characteristic iminium ions and diagnostic ions at $m / z, 91$ and $m / z$ 105. For EI mass spectra, radical-directed $\alpha$ cleavages result in dominant iminium ions. In contrast to $\alpha$-pyrrolidinophenone synthetic cathinones, MS/MS of protonated $\mathrm{N}$-alkylated synthetic cathinones provided abundant radical losses from both the $\mathrm{N}$-alkylated and aliphatic side chains, a dominant loss of $\mathrm{H}_{2} \mathrm{O}$ for $2^{\circ}$ amines and the formation of abundant alkylphenones for $3^{\circ}$ amines. These findings help advance our current understanding of the MS/MS analysis of synthetic cathinones, and they help analysts better understand and defend their observations and interpretations in existing and future casework. 
For FRC's, a combination of isotope labeling, HRMS and $\mathrm{MS}^{\mathrm{n}}$ experiments identified a novel isobaric product ion at $m / z 188$, which is elementally distinct from the two previous known isobars at $m / z, 188$ and forms through an intermediate product ion at $m / z 216$ (for fentanyl). These studies also confirmed the pathways through which the three nominal isobars are formed and how substitutions to the aniline ring and amide moieties result in remarkably conserved fragmentation pathways. In contrast, substitutions to the piperidine ring, the $N$-alkyl chain, and the cyclic substituent of FRCs resulted in distinct differences in fragmentation pathways, the abundance of which is related to the identity of the specific substitution. For example, the presence of a hydroxyl group on the $N$-alkyl chain results in the MS/MS spectrum being dominated by the neutral loss of water, whereas the presence of a methyl group favors the formation of the tropylium ion. By understanding the fragmentation behavior of fentanyl and the impact of substitutions to the core fentanyl structure, toxicologists and seized drug analysts will be better prepared to identify emerging FRCs, which are increasingly common and deadly adulterants in the growing opioid epidemic.

The final major contribution from this work was the comparison between in-source CID and beam-type CID experiments of the same synthetic cathinones and FRCs on the same instrument. Whereas the relative abundance of certain fragments were often readily distinguishable between in-source CID and beam-type CID, the fragment $\mathrm{m} / \mathrm{z}$ values and the overall pattern of fragmentation were sufficiently consistent that the spectra from the two different activation methods could serve as proxies for one another. However, because in-source CID involves fragmentation of all precursors from the source region of the mass spectrometer rather than through isolation and fragmentation in the collision cell, caution should be used when analyzing potential mixtures or complex biological samples where strict control of precursor ions present in the source region may not be possible. 


\section{Dedication}

I dedicate this work to my loving parents, Frank Wayne and Glenda Kay Davidson, and my fiancé, Heather Amber Winter. Throughout my educational experience the three of you have always provided the necessary guidance, support, and love. This dissertation is the culmination of an educational journey that began through a desire to provide a future greater than one's own through dedication and sacrifice. I wouldn't be here today without the significant contributions of these three tremendous individuals.

"Sometimes we will die and sometimes we will fly away

Either way you're by my side until my dying days

And if I'm not there and I'm far away

I said, 'Don't be afraid'."

Taxi Cab - Twenty One Pilots 


\section{Acknowledgements}

Over the last five years, Dr. Glen P. Jackson has guided my development not only as a teacher and researcher, but also as an individual. Dr. Jackson's passion for teaching and research have helped to ignite my own passion in these areas. Likewise, Dr. Jackson has spent countless hours sharing his experiences in academia and being transparent about professional development so that I may one day lead my own research group. As an individual, Dr. Jackson has helped me to identify what is most important in my life and how best to find balance in my pursuits. The core values of my teaching philosophy and research direction are a direct reflection of my time with Dr. Jackson.

I would also like to thank Dr. Luis E. Arroyo, Dr. Tatiana Trejos, Dr. Stephen J. Valentine, and Dr. Suzanne Bell for their contributions over the course of my dissertation development. Although Dr. Suzanne Bell was only present for the proposal portion of my dissertation, she was still a contributing member to the successful completion of this work. I am thankful for my committee members' willingness to always be available to provide guidance or assistance throughout the course of my dissertation.

I am also thankful for my fellow lab members in the Jackson Group who have assisted me over the past five years, as well as my colleagues in the Department of Forensic and Investigative Science. I cannot possibly list everyone, but in short, the graduate students include: Dr. Pengfei Li, Dr. Mayara Matos, Ashley Cochran, Korina Menking-Hoggatt, Halle Edwards, Praneeth Mario Menidis, Zachary Sasiene, Caitlyn Wensel, Alia Hacker, and Alex Adeoye and the undergraduates that I mentored for at least an academic year including: Isaac Willis, Sarah Chaffman, and Samantha Mehnert.

Most importantly, I would like to thank my family for their continued love and support throughout my education. My academic journey began at Shippensburg University where I found both my passion for chemistry and my best friend who would accompany me on this journey. Much of my academic success has been a direct result of the opportunities afforded to me by my parents and the encouragement of those around me. I would not be here today without this continued support and encouragement every step along the way. 
Table of Contents

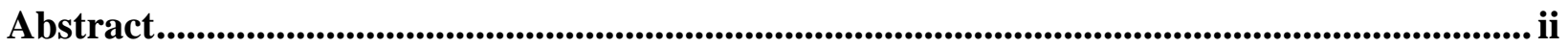

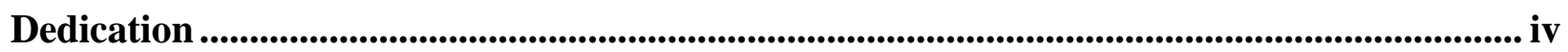

Acknowledgements ................................................................................................................................ V

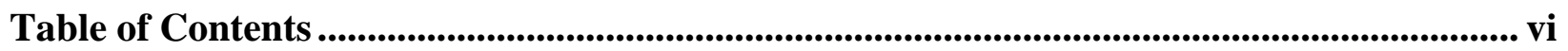

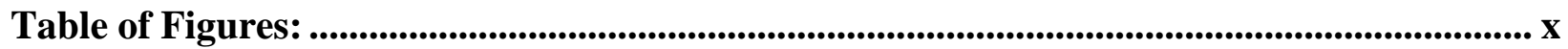

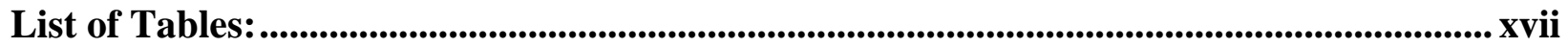

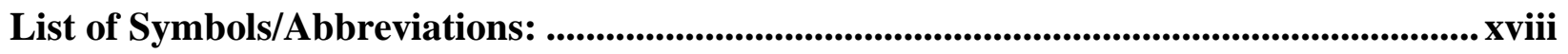

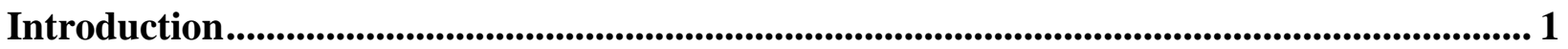

Chapter 1: Identification of novel fragmentation pathways and fragment ion structures in the tandem mass spectra of protonated synthetic cathinones..................................................... 7

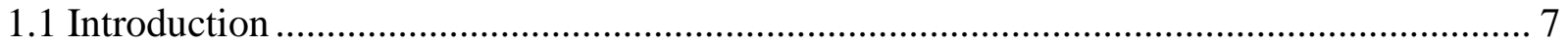

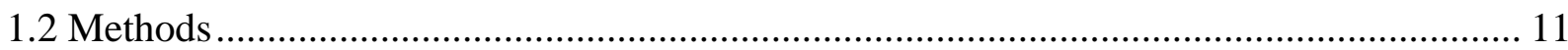

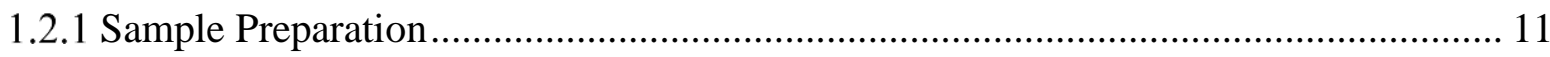

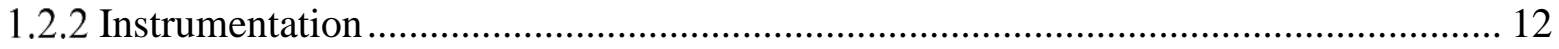

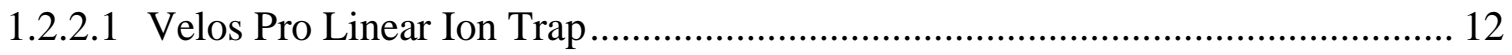

1.2.2.2 Agilent Technologies 6538 UHD Accurate-Mass Quadrupole Time-of-Flight (Q-

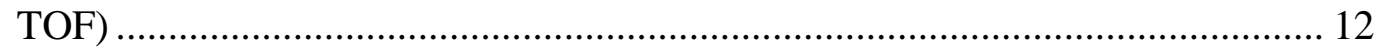

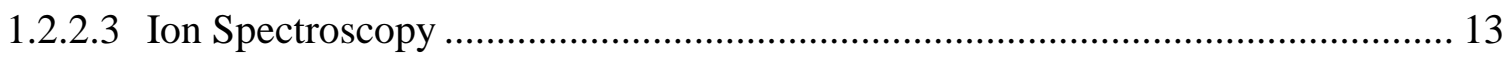

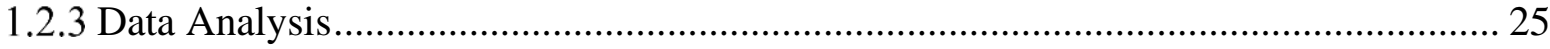

1.2.3.1 Mass Spectral Interpretation and Mechanisms ............................................. 25

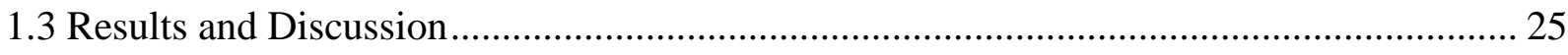

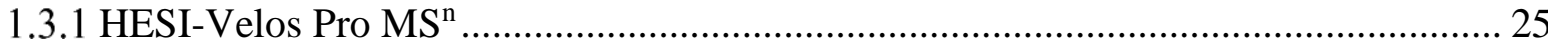

1.3.1 High-Resolution Mass Spectrometry (HRMS) Measurements using ESI-Q-TOF ..... 47

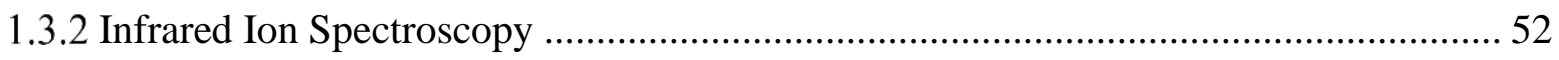

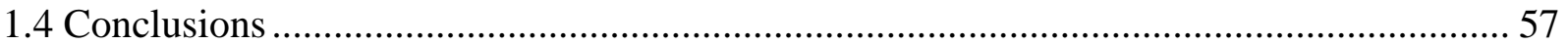

Chapter 2: Fragmentation pathways of $\alpha$-pyrrolidinophenone synthetic cathinones and their application to the identification of emerging synthetic cathinone derivatives.............. 59

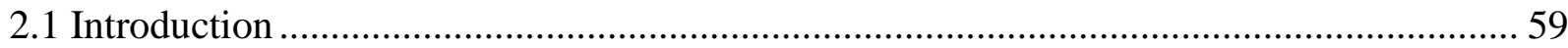




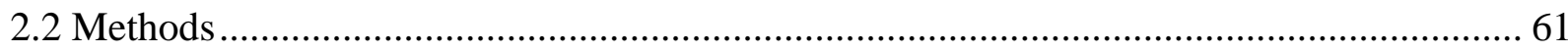

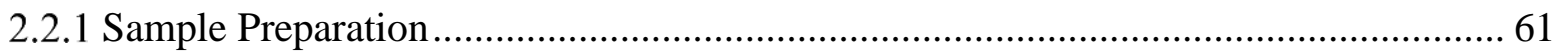

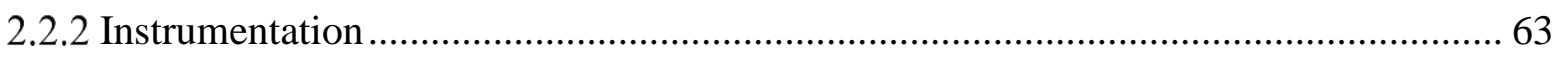

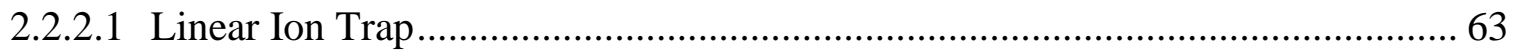

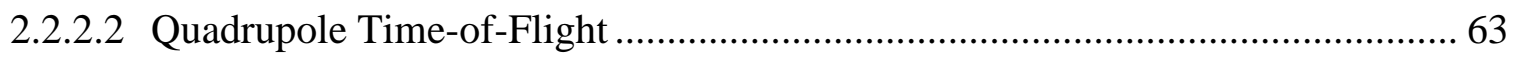

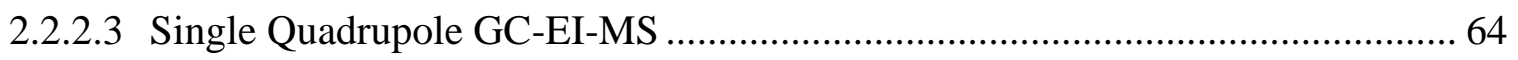

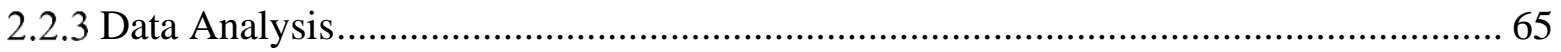

2.2.3.1 Mass Spectral Interpretation and Mechanisms................................................... 65

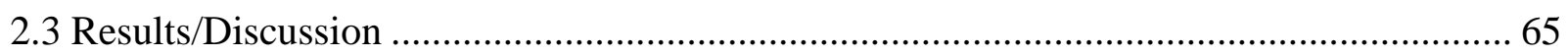

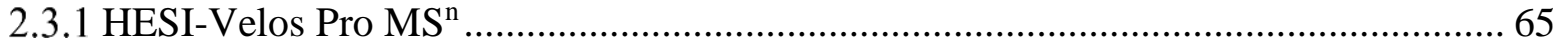

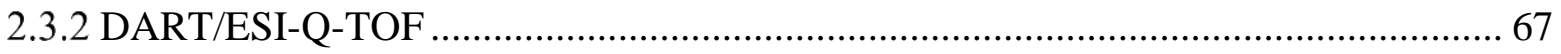

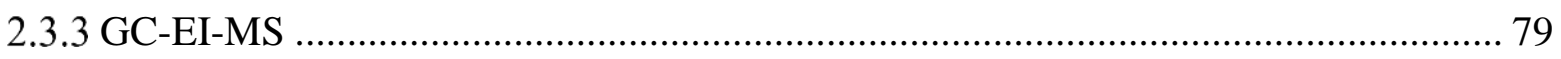

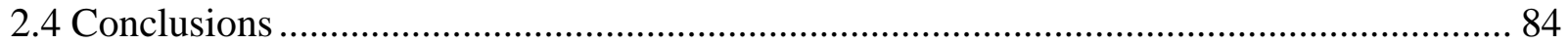

Chapter 3: Fragmentation pathways of odd- and even-electron $N$-alkylated synthetic cathinones ........................................................................................................................................ 86

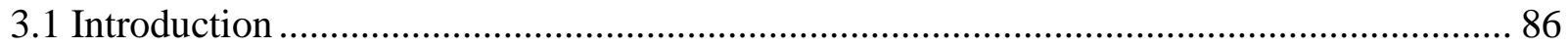

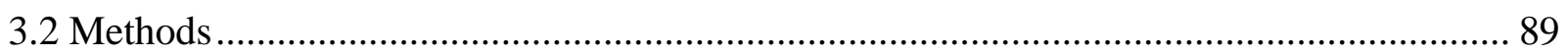

3.2.1 Sample Preparation ................................................................................................. 89

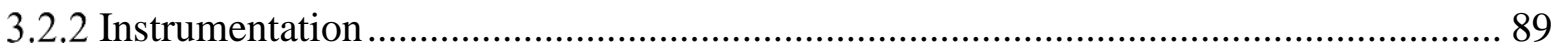

3.2.2.1 Linear Ion Trap....................................................................................... 89

3.2.2.2 Quadrupole Time-of-Flight ............................................................................. 90

3.2.2.3 Single Quadrupole GC-EI-MS ...................................................................... 90

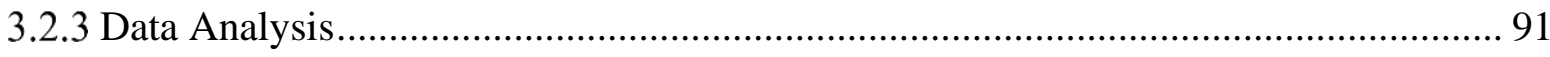

3.2.3.1 Mass Spectral Interpretation and Mechanisms ................................................... 91

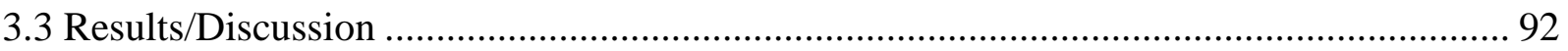

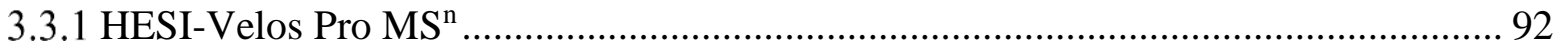

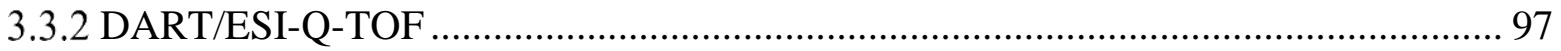

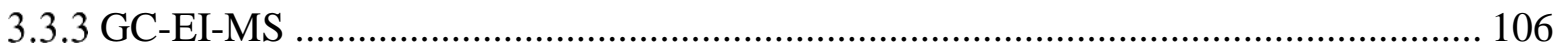

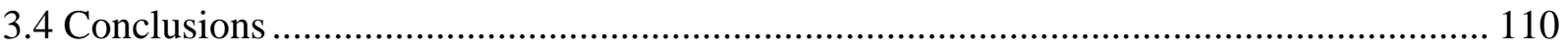

Chapter 4: The characterization of isobaric product ions of fentanyl using multi-stage mass spectrometry, high-resolution mass spectrometry and isotopic labeling................................ 113 


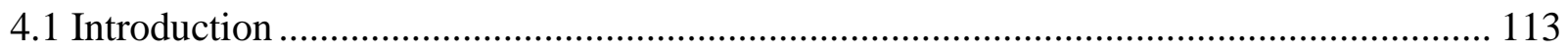

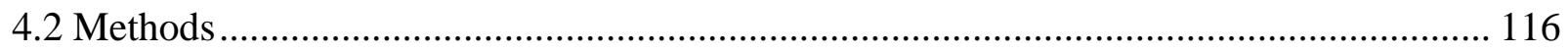

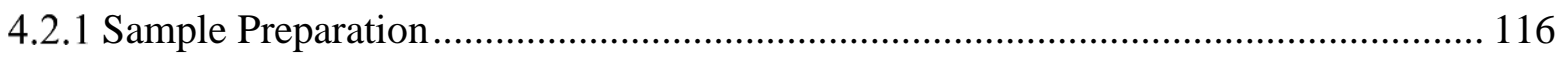

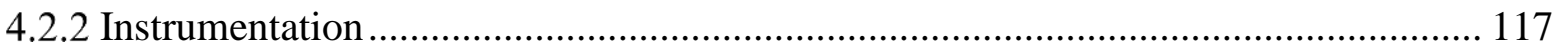

4.2.2.1 Thermo Scientific Velos Pro Linear Ion Trap (LIT) ……………………….... 117

4.2.2.2 Agilent Technologies 6538 UHD Accurate-Mass Quadrupole Time-of-Flight (Q-

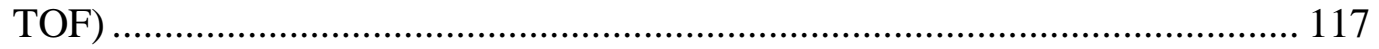

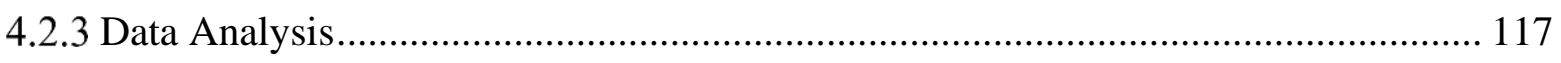

4.2.3.1 Mass Spectral Interpretation and Mechanisms............................................... 118

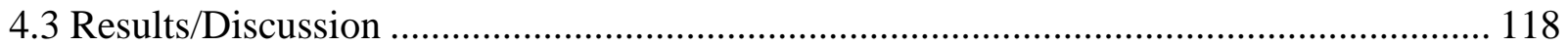

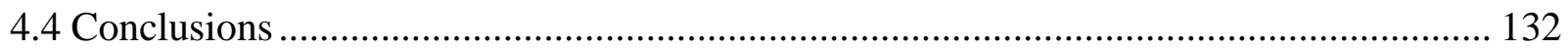

Chapter 5: The influence of chemical modifications on the fragmentation behavior of fentanyl and fentanyl-related compounds in electrospray ionization tandem mass spectrometry ........................................................................................................................................ 134

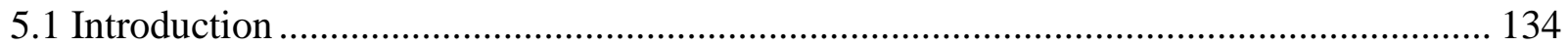

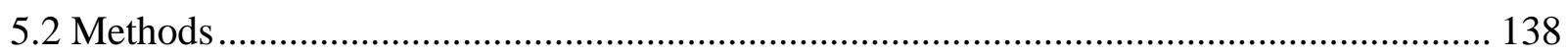

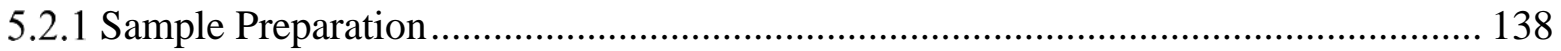

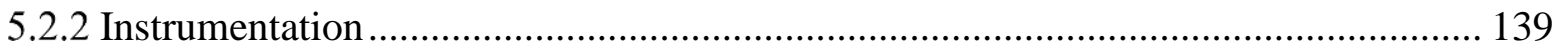

5.2.2.1 Thermo Scientific Velos Pro Linear Ion Trap (LIT) ....................................... 139

5.2.2.2 Agilent Technologies 6538 UHD Accurate-Mass Quadrupole Time-of-Flight (Q-

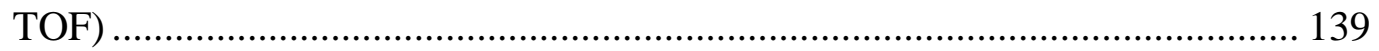

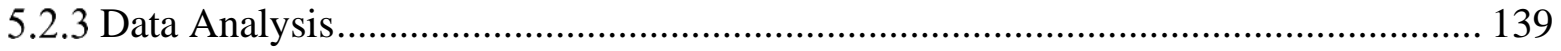

5.2.3.1 Mass Spectral Interpretation and Mechanisms.............................................. 140

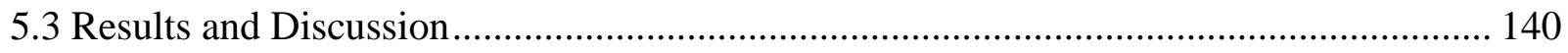

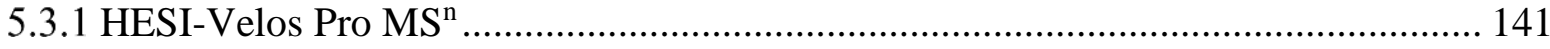

5.3.2 Accurate-Mass with HRMS Q-TOF.................................................................. 147

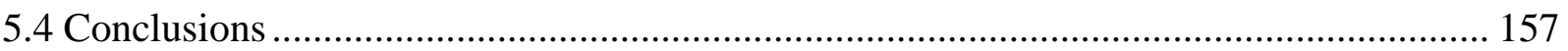

Chapter 6: Comparison of in-source collision-induced dissociation and beam-type collisioninduced dissociation of synthetic cathinones and fentanyl analogs using a high-resolution quadrupole time-of-flight (Q-TOF) mass spectrometer........................................................... 159

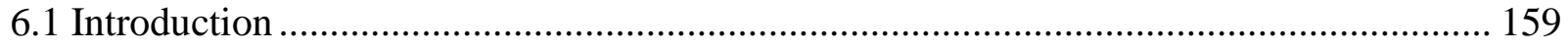

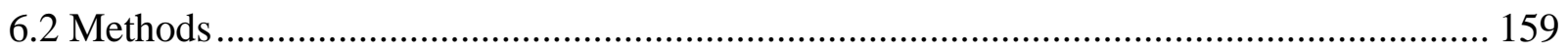




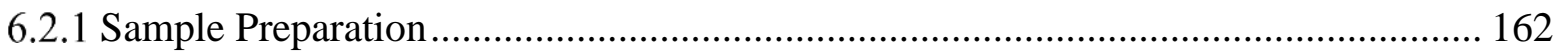

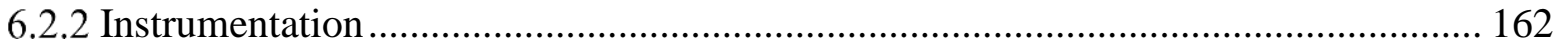

6.2.2.1 Agilent Technologies 6538 UHD Accurate-Mass Quadrupole Time-of-Flight (Q-

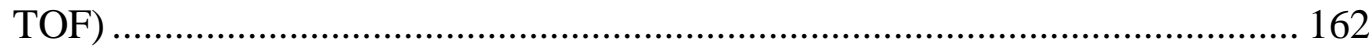

6.2.2.2 Thermo Scientific Velos Pro Linear Ion Trap (LIT) ......................................... 163

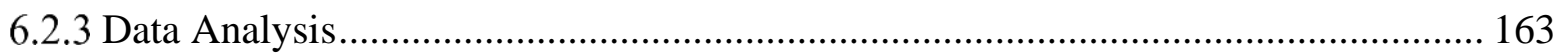

6.3 Results and Discussiom.............................................................................................. 164

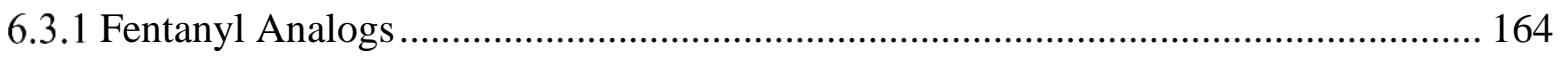

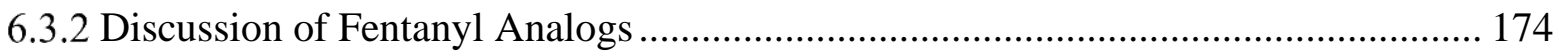

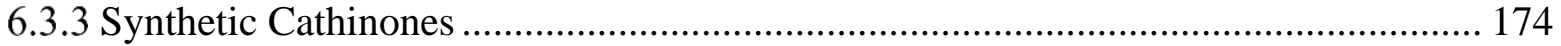

6.3.4 Discussion of Synthetic Cathinones ................................................................... 189

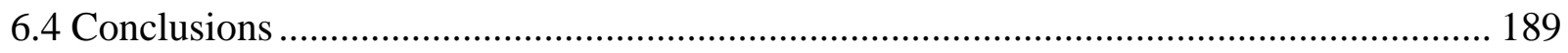

Conclusions and Future Work................................................................................ 192

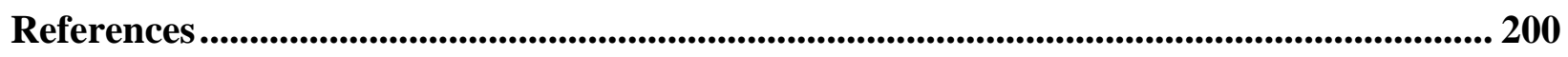

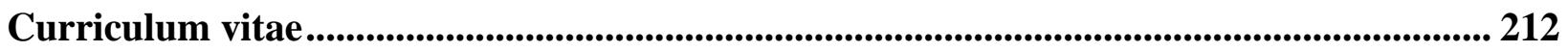




\section{Table of Figures:}

Figure 1.1. Optimized geometries of seven possible isomeric structures of the fragment at $\mathrm{m} / \mathrm{z}$ 119. Relative energies and theoretical IR spectra are calculated at the B3LYP/6-311+G(d,p) level of theory. Energies are reported in $\mathrm{kJ} / \mathrm{mol}$ relative to structure 119a. Calculated IR (blue) are overlapped with the experimental spectrum (orange) of the $\mathrm{m} / \mathrm{z} 119$ fragment.

Figure 1.2. Optimized geometries of nine possible isomeric structures of the fragment at $\mathrm{m} / \mathrm{z}$ 133. Relative energies and theoretical IR spectra are calculated at the B3LYP/6-311+G(d,p) level of theory. Energies are reported in $\mathrm{kJ} / \mathrm{mol}$ relative to structure 133a. Calculated IR (blue) are overlapped with the experimental spectrum (orange) of the $m / z 133$ fragment. 16

Figure 1.3. Tandem mass spectra of $\alpha-\mathrm{PVP}$ : a) $\mathrm{MS}^{2}$ product ion spectrum of the $[\mathrm{M}+\mathrm{H}]^{+}$ molecular ion (35\% NCE); b) $\mathrm{MS}^{3}$ product ion spectrum of the product ion at $\mathrm{m} / z 161$ (30\% NCE) showing the formation of product ions at $m / z$ 143, 133, 119, 105, and 91; c) MS $\mathrm{M}^{4}$ product ion spectrum of the secondary product ion at $\mathrm{m} / z 119$ (30\% NCE) showing the formation of only the tropylium ion at $m / z 91$. Evidence for the phthalane structure shown in panel b) is provided by ion spectroscopy and DFT calculations in section 1.3.2.

Figure 1.4. Tandem mass spectra of ${ }^{13} \mathrm{C}$-carbonyl labeled $\alpha$-PVP: a) $\mathrm{MS}^{2}$ product ion spectrum of the $[\mathrm{M}+\mathrm{H}]^{+}$molecular ion $(35 \% \mathrm{NCE})$; b) $\mathrm{MS}^{3}$ spectrum of the intermediate ion at $\mathrm{m} / z 162$ (30\% NCE); c) $\mathrm{MS}^{4}$ spectrum of the intermediate ion at $\mathrm{m} / z 120$ (30\% NCE) showing the formation of only the secondary product ion at $\mathrm{m} / \mathrm{z}$ 91. Evidence for the phthalane structures in panels a) and b) are provided by ion spectroscopy and DFT calculations in section 1.3.2. ........ 29 Figure 1.5. Tandem mass spectra of ${ }^{18} \mathrm{O}-\alpha-\mathrm{PVP}:$ a) $\mathrm{MS}^{2}$ product ion spectrum of the $[\mathrm{M}+\mathrm{H}]^{+}$ molecular ion (35\% NCE); b) $\mathrm{MS}^{3}$ spectrum of the product ion at $\mathrm{m} / z 163(30 \% \mathrm{NCE})$ showing the formation of product ions at $m / z, 143,135,133,121,107$ and $91 ; \mathrm{c}$ ) $\mathrm{MS}^{4}$ spectrum of the secondary product ion at $\mathrm{m} / \mathrm{z} 121$ (30\% NCE) showing only the formation of tropylium ion at $\mathrm{m} / \mathrm{z}$ 91. Evidence for the phthalane structures in panels a) and b) are provided by ion spectroscopy

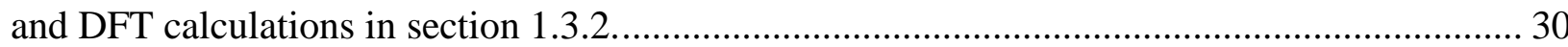

Figure 1.6. Tandem mass spectra of ${ }^{13} \mathrm{C}-\alpha$-carbon $\alpha$-PVP: a) $\mathrm{MS}^{2}$ product ion spectrum of the $[\mathrm{M}+\mathrm{H}]^{+}$molecular ion $(35 \% \mathrm{NCE})$; b) $\mathrm{MS}^{3}$ spectrum of the intermediate ion at $\mathrm{m} / z .162(30 \%$ $\mathrm{NCE})$; c) $\mathrm{MS}^{4}$ spectrum of the intermediate ion at $\mathrm{m} / \mathrm{z} 120$ (30\% NCE) showing the formation of only the product ion at $\mathrm{m} / \mathrm{z}$ 92. Evidence for the phthalane structures in panels a) and b) are provided by ion spectroscopy and DFT calculations in section 1.3.2.

Figure 1.7. Proposed mechanisms for the formation of $\mathrm{m} / \mathrm{z} 134,133$, and 120 product ions from ${ }^{13} \mathrm{C}$-carbonyl carbon- $\alpha$-PVP. The phthalane structures at $\mathrm{m} / \mathrm{z} 120$ and $\mathrm{m} / \mathrm{z} 134$ were confirmed by ion spectroscopy and DFT calculations (see Figure 1.22 and Figure 1.23). 33 Figure 1.8. Proposed mechanisms for the formation of product ions at $\mathrm{m} / \mathrm{z} 91$ and $\mathrm{m} / \mathrm{z} 92$ from the intermediate at $\mathrm{m} / \mathrm{z}, 120$ for $\mathrm{MS}^{4}$ based on ${ }^{13} \mathrm{C}$ isotopic labeling of the carbonyl carbon and the $\alpha$-carbon for $\alpha$-PVP. 35

Figure 1.9. $\mathrm{MS}^{3}$ product ion mass spectra of: a) the product ion at $m / z 134$ (30\% NCE) showing the formation of product ions at $m / z 106,105$, and 92 from ${ }^{13} \mathrm{C}$-carbonyl carbon- $\alpha-\mathrm{PVP}$ and $\mathrm{b}$ ) 
the product ion at $m / z 133(30 \%$ NCE) showing the presence of only the product ions at $\mathrm{m} / z 105$ and $m / z, 91$ from ${ }^{13} \mathrm{C}$-carbonyl carbon- $\alpha$-PVP. 36

Figure 1.10. Proposed mechanisms for the formation of the product ions from the intermediate ions at $m / z, 134$ and $m / z, 133$ as observed from the $\mathrm{MS}^{3}$ analysis for ${ }^{13} \mathrm{C}$-carbonyl carbon- $\alpha$-PVP.

Figure 1.11. $\mathrm{MS}^{3}$ product ion mass spectra of the product ion at $m / z 134$ (30\% NCE) showing the formation of product ions at $m / z, 106,105,92$ and 91 from ${ }^{13} \mathrm{C}-\alpha$-carbon- $\alpha$-PVP. 37

Figure 1.12. Proposed mechanisms for the formation of the product ions at $m / z 106,105,92$, and 91 from the $\mathrm{MS}^{3}$ analysis of the intermediate ion at $m / z .134$ from ${ }^{13} \mathrm{C}$ - $\alpha$-carbon- $\alpha$-PVP. .......... 38

Figure 1.13. Tandem mass spectra of $\alpha-\mathrm{PVP}-\mathrm{d}_{7}$ : a) $\mathrm{MS}^{2}$ product ion spectrum of the $[\mathrm{M}+\mathrm{H}]^{+}$ molecular ion $(35 \% \mathrm{NCE})$; b) $\mathrm{MS}^{3}$ product ion spectrum of the product ion at $\mathrm{m} / \mathrm{z} 168(30 \%$ NCE) showing the formation of secondary product ions at $m / z, 140,122,121,120,94,93$, and 92; c) $\mathrm{MS}^{4}$ product ion spectrum of the intermediate at $\mathrm{m} / z, 140$ (30\% NCE) showing the formation of secondary product ions at $m / z, 92,93$, and 94 .

Figure 1.14. Tandem mass spectra of: a) $\alpha$-PBP (35\% NCE), b) $\alpha$-PPP (30\% NCE), and c) $\alpha-$ PVP-methyl at the $\alpha$-carbon (35\% NCE) demonstrating the presence of the tropylium ion at $\mathrm{m} / z$ 91 for $\alpha$-PBP, absence for $\alpha$-PPP, and essential absence $(<2 \%)$ for $\alpha$-PVP-methyl ( $\alpha$-carbon).. 42 Figure 1.15. Tandem mass spectra of 3,4-MDPV- $\mathrm{d}_{8}$ : a) $\mathrm{MS}^{2}$ product ion spectrum of the $[\mathrm{M}+\mathrm{H}]^{+}$molecular ion (35\% NCE); b) $\mathrm{MS}^{3}$ product ion spectrum of the product ion at $\mathrm{m} / \mathrm{z} 205$ (35\% NCE) showing the formation of secondary product ions at $\mathrm{m} / \mathrm{z} 177,163$ and 135; and c) $\mathrm{MS}^{4}$ product ion spectrum of the product ion at $\mathrm{m} / z 177$ (35\% NCE) showing the formation of the tropylium ion derivative at $\mathrm{m} / \mathrm{z} 135$.

Figure 1.16. Tandem mass spectra of positional isomers highlighting the impact of substituent location as demonstrated by a) 3,4-MDPV (35\% NCE) and b) 2,3-MDPV (35\% NCE) demonstrating the differences in fragmentation based on location of the methylenedioxy substituent.

Figure 1.17. Selected fragmentation pathways and corresponding flux for: a) $\alpha$-PBP, b) $\alpha-P V P$, and c) PV8. The percentages shown at each level of $\mathrm{MS}^{\mathrm{n}}$ provide the ion's abundance relative to the summed ion abundance of the product ion spectrum at that level. For example, $\mathrm{m} / \mathrm{z} 147$ is the base peak (100\% peak height) in the $\mathrm{MS}^{2}$ product ion spectrum of $\alpha$-PBP and $55 \%$ of the summed product ion spectrum. 46

Figure 1.18. Tandem mass spectra of $\alpha$-PVP showing: a) the fragmentation observed on the QTOF mass spectrometer with a $25 \mathrm{eV}$ collision energy and b) a zoomed-in view of product ions at $\mathrm{m} / \mathrm{z} 133.0611$ and $\mathrm{m} / \mathrm{z} 133.0970$ with a $35 \mathrm{eV}$ collision energy.

Figure 1.19. Tandem mass spectra of PV8 $(25 \mathrm{eV})$ showing the conserved nature of the loss of the pyrrolidine moiety with the Q-TOF mass spectrometer and the propensity to form the tropylium ion.

Figure 1.20. Tandem mass spectrum of $3,4-\mathrm{MDPV}$ ( $25 \mathrm{eV}$ collision energy) showing the propensity to form the substituted tropylium ion is conserved in a wide variety of substituted cathinones. 
Figure 1.21. Tandem mass spectra of ${ }^{13} \mathrm{C}$-MPHP ( $25 \mathrm{eV}$ collision energy) showing the dominant abundance of $m / z, 105.0727$ for the $\mathrm{C}_{8} \mathrm{H}_{9}{ }^{+}$ion relative to $m / z, 91.0565$ for the $\mathrm{C}_{7} \mathrm{H}_{7}{ }^{+}$tropylium ion. The structure of the ion at $\mathrm{m} / z, 105$ could equally take the form of a methyl-substituted tropylium ion.

Figure 1.22. Comparison of experimental gas-phase IR ion spectroscopy and DFT theoretical IR spectra for fragments of protonated $\alpha$-PVP: a) proposed 119a structure demonstrating a good alignment between experimental and predicted spectra and b) proposed 119d structure demonstrating a poor alignment between the experimental and predicted spectra. 54 Figure 1.23. Comparison of experimental gas-phase IR ion spectroscopy and DFT calculated IR spectra for fragments of protonated $\alpha$-PVP: a) proposed 133a' structure demonstrating a good alignment between experimental and predicted spectra and b) proposed 133c structure demonstrating a poor alignment between the experimental and predicted spectra. 55

Figure 2.1. Tandem mass spectra of $\alpha$-PVP-d d $_{8}$ a) $\mathrm{MS}^{2}$ product ion spectrum of the $[\mathrm{M}+\mathrm{H}]^{+}$ molecular ion at $m / z 240(35 \% \mathrm{NCE})$; b) $\mathrm{MS}^{3}$ product ion spectrum for the pathway $\mathrm{m} / \mathrm{z}$ $240 \rightarrow 161 \rightarrow$ at $30 \%$ NCE showing the formation of secondary product ions at $m / z, 143,133,119$, 105 and 91 ; c) $\mathrm{MS}^{3}$ product ion spectrum for the pathway $m / z, 240 \rightarrow 134 \rightarrow$ at $30 \%$ NCE showing the formation of product ions at $m / z, 106,105$ and 92 .

Figure 2.2. Tandem mass spectra of 4-MeO- $\alpha$-PVP collected on the same Q-TOF HRMS instrument using a) ESI with a $25 \mathrm{eV}$ collision energy and b) DART ionization with a $25 \mathrm{eV}$ collision energy.

Figure 2.3. Tandem mass spectra of 3,4-MDPV collected on the same Q-TOF HRMS instrument using a) ESI with a $25 \mathrm{eV}$ collision energy and b) DART ionization with a $25 \mathrm{eV}$ collision

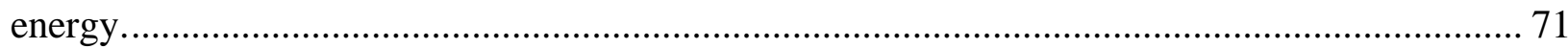

Figure 2.4. Tandem mass spectra of ${ }^{13} \mathrm{C}$-MPHP collected on the same Q-TOF HRMS instrument using a) ESI with a $25 \mathrm{eV}$ collision energy and b) DART ionization with a $25 \mathrm{eV}$ collision

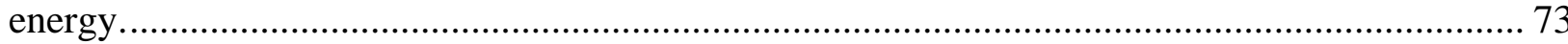

Figure 2.5. Proposed general fragmentation pathways for protonated $\alpha$-pyrrolidinophenone synthetic cathinones undergoing tandem MS. The model compound is $\alpha$-PVP where $\mathrm{X}=\mathrm{H}$ and the $m / z$ values that are specific to $\alpha$-PVP indicated with an asterisk $(*)$. .............................. 75 Figure 2.6. Tandem mass spectra of: a) $\alpha$-PBP (35\% NCE), b) $\alpha$-PVP (35\% NCE), and c) PV8 (35\% NCE) demonstrating the effect of the alkyl chain length on the distribution of product ions. 77

Figure 2.7. Full scan mass spectra of a) ${ }^{13} \mathrm{C}-\alpha$-PPP and b) ${ }^{13} \mathrm{C}-3,4-\mathrm{MDPV}$ collected with GC-EIMS.

Figure 2.8. Full scan mass spectra of ${ }^{13} \mathrm{C}-\mathrm{PV} 8$ collected with GC-EI-MS.............................. 82 Figure 2.9. Proposed general mechanisms for the fragmentation of $\alpha$-pyrrolidinophenone synthetic cathinones with EI-MS (adapted from references [30, 60, 65]). The model compound is $\alpha$-PVP.

Figure 3.1. Product ion mass spectra of pentedrone on the LIT: a) product ion spectrum of the $[\mathrm{M}+\mathrm{H}]^{+}$molecular ion $(30 \% \mathrm{NCE})$; b) product ion spectrum of the product ion at $\mathrm{m} / \mathrm{z} 174(30 \%$ 
NCE) showing the formation of secondary product ions at $m / z, 159,145,132$, and 131; c) product ion spectrum of the primary product ion at $\mathrm{m} / \mathrm{z} 161$ (30\% NCE) showing the characteristic valerophenone ion fragmentation.

Figure 3.2. Product ion mass spectra of pentylone: a) product ion spectrum of the $[\mathrm{M}+\mathrm{H}]^{+}$ molecular ion (30\% NCE); b) product ion spectrum of the product ion at $m / z 218$ (30\% NCE) showing the formation of secondary product ions at $\mathrm{m} / \mathrm{z} 176,175,160$, and 146; c) product ion spectrum of the primary product ion at $\mathrm{m} / z 205$ (30\% NCE) showing the characteristic methylenedioxy valerophenone ion fragmentation. 95

Figure 3.3. Product ion mass spectra of dibutylone- $d_{3}$ on the LIT: a) product ion spectrum of the $[\mathrm{M}+\mathrm{H}]^{+}$molecular ion $(30 \% \mathrm{NCE})$; b) product ion spectrum of the primary product ion at $\mathrm{m} / \mathrm{z}$ 194 (30\% NCE) showing the formation of product ions at $\mathrm{m} / z, 166,164$, and 136; c) product ion spectrum of the primary product ion at $\mathrm{m} / \mathrm{z} 89$ (30\% NCE) showing the loss of both a methyl radical and a deuterated methyl radical.

Figure 3.4. Tandem mass spectra of methcathinone- $d_{3}$ collected on the Q-TOF with both a) ESI and b) DART ionization with a collision energy of $25 \mathrm{eV}$ and skimmer voltage of $65 \mathrm{~V}$. The ESI spectrum was collected with a fragmentor voltage of $175 \mathrm{~V}$, whereas the DART spectrum was collected with a fragmentor voltage of $150 \mathrm{~V}$.

Figure 3.5. Tandem mass spectra of diethylpropion- $d_{10}$ collected on the Q-TOF with both a) ESI and b) DART ionization with a collision energy of $25 \mathrm{eV}$. The ESI spectrum was collected with a fragmentor voltage of $225 \mathrm{~V}$, whereas the DART spectrum was collected with a fragmentor voltage of $150 \mathrm{~V}$, which were both collected with a skimmer voltage of $65 \mathrm{~V}$. 101

Figure 3.6. Tandem mass spectra of eutylone-d5 collected with both a) ESI and b) DART ionization with a collision energy of $25 \mathrm{eV}$. The ESI spectrum was collected with a fragmentor voltage of $225 \mathrm{~V}$, whereas the DART spectrum was collected with a fragmentor voltage of 150 $\mathrm{V}$, which were both collected with a skimmer voltage of $65 \mathrm{~V}$

Figure 3.7. Proposed mechanisms for fragmentation pathways of $\mathrm{N}$-alkylated synthetic cathinones. The model compound is pentedrone (aliphatic group $=\mathrm{CH}_{3}, N$-alkyl group $=\mathrm{CH}_{3}$, $\mathrm{X}=\mathrm{H}$ ) with ions specific pentedrone indicated with an asterisk $(*)$; different functional groups, $\mathrm{X}$, will shift the product ion $\mathrm{m} / \mathrm{z}$ values accordingly.

Figure 3.8. Full scan mass spectra of ${ }^{13} \mathrm{C}$-ethylone (isotopically labeled with on the carbonyl carbon) collected with GC-EI-MS.

Figure 3.9. Full scan mass spectra of $\alpha$-propylaminopentiophenone collected with GC-EI-MS.

Figure 3.10. Full scan mass spectra of ${ }^{13} \mathrm{C}$-benzedrone collected with GC-EI-MS. 109

Figure 3.11. Proposed EI-MS fragmentation mechanisms for the $N$-alkylated class of synthetic cathinones (adapted from $[60,75]$ ). The model compound is pentedrone (aliphatic group $=\mathrm{CH}_{3}$, $N$-alkyl group $=\mathrm{CH}_{3}, \mathrm{X}=\mathrm{H}$ ) with ions specific to pentedrone indicated with an asterisk (*)..... 110 Figure 4.1 [87]. Generic chemical structure of fentanyl highlighting regions of substitutions for fentanyl analogs. 
Figure 4.2. Tandem mass spectrum of protonated fentanyl using CID in a linear ion trap mass spectrometer (35\% NCE).

Figure 4.3. Product ion mass spectra of protonated fentanyl collected under different conditions: a) $\mathrm{MS}^{3}$ product ion spectrum for the transition $\mathrm{m} / \mathrm{z} 337 \rightarrow 281 \rightarrow$ at $35 \% \mathrm{NCE}$, and b) $\mathrm{MS}^{4}$ product ion spectrum for the transition $m / z \quad 337 \rightarrow 281 \rightarrow 188 \rightarrow$ at $33 \%$ NCE.

Figure 4.4. Proposed mechanisms for the formation of different product ions from two isobaric intermediates at $m / z 188$ [97]. Pathway a) leads to the formation of product ions at $m / z 134,160$, and 120, whereas pathway b) leads to the formation of product ions at $m / z, 146,132$, and 105.121 Figure 4.5. Product ion mass spectra of protonated fentanyl collected under different conditions: a) $\mathrm{MS}^{3}$ product ion spectrum for the transition $\mathrm{m} / \mathrm{z} 337 \rightarrow 216 \rightarrow$ at $35 \% \mathrm{NCE}$, and b) $\mathrm{MS}^{4}$ product ion spectrum for the transition $m / z, 337 \rightarrow 216 \rightarrow 188 \rightarrow$ at $25 \%$ NCE. 122

Figure 4.6. Comparison of $\mathrm{MS}^{2}$ of: a) the in-source CID generated intermediate product ion at $\mathrm{m} / \mathrm{z} 216.1300(25 \mathrm{eV})$ and b) the in-source CID generated intermediate product ion at $\mathrm{m} / \mathrm{z}$ $281.2000(25 \mathrm{eV})$ for fentanyl.

Figure 4.7. Proposed mechanisms for the formation of the novel product ion at $\mathrm{m} / \mathrm{z} 188$ from the intermediate product ion at $m / z 216$. 124 Figure 4.8. Product ion mass spectra of protonated fentanyl collected under different conditions: a) $\mathrm{MS}^{3}$ product ion spectrum for the transition $\mathrm{m} / \mathrm{z} 337 \rightarrow 244 \rightarrow$ at $25 \% \mathrm{NCE}$, and b) $\mathrm{MS}^{4}$ product ion spectrum for the transition $\mathrm{m} / \mathrm{z} 337 \rightarrow 244 \rightarrow 188 \rightarrow$ at $25 \% \mathrm{NCE}$. 126

Figure 4.9. Proposed mechanisms for the formation of the product ion at $m / z 188$ from the intermediate product ion at $m / z 244$.

Figure 4.10. Tandem mass spectrum of fentanyl $(25 \mathrm{eV})$ and showing the product ion at $\mathrm{m} / \mathrm{z}$ 190.1249, which supports the proposed $\mathrm{C}_{12} \mathrm{H}_{16} \mathrm{ON}^{+}$elemental formula.

Figure 4.11. Product ion mass spectra of protonated fentanyl- $\mathrm{d}_{5}$ collected under different conditions: a) $\mathrm{MS}^{2}$ product ion spectrum of the precursor at $\mathrm{m} / z 342$ at $35 \% \mathrm{NCE}$, b) $\mathrm{MS}^{3}$ product ion spectrum for the transition $m / z, 337 \rightarrow 286 \rightarrow$ at $25 \% \mathrm{NCE}$, and c) $\mathrm{MS}^{3}$ product ion spectrum for the transition $m / z, 337 \rightarrow 221 \rightarrow$ at $25 \%$ NCE.

Figure 4.12. $\mathrm{MS}^{2}$ of the in-source CID generated intermediate product ion at $\mathrm{m} / \mathrm{z} 221.1700$ (25 $\mathrm{eV}$ ) for fentanyl- $\mathrm{d}_{5}$ highlighting the incorporation the deuterated aniline moiety in the product ions at $m / z 221.1709,193.1407,165.1464$, and 137.1147

Figure 4.13. Product ion mass spectra of protonated 4-ANPP: a) $\mathrm{MS}^{2}$ product ion spectrum of protonated precursor ion at $m / z 281(35 \% \mathrm{NCE})$ and b) $\mathrm{MS}^{3}$ product ion spectrum for the

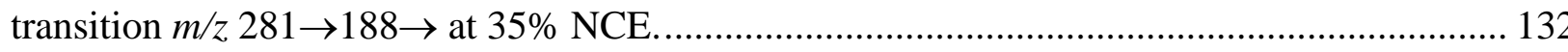

Figure 5.1. Generic chemical structure of fentanyl-related compounds (FRCs).................... 135 Figure 5.2. Tandem mass spectra of ortho-methylfentanyl: a) $\mathrm{MS}^{2}$ product ion spectrum of the $[\mathrm{M}+\mathrm{H}]^{+}$molecular ion $\left.(30 \% \mathrm{NCE}) ; \mathrm{b}\right) \mathrm{MS}^{4}$ product ion spectrum of the product ion at $\mathrm{m} / \mathrm{z} 188$ (35\% NCE) showing the formation of product ions at $\mathrm{m} / \mathrm{z}, 160,146,132$ and 105, among others; c) $\mathrm{MS}^{3}$ product ion spectrum of the primary product ion at $m / z 230$ (30\% NCE) showing the formation of $\mathrm{m} / \mathrm{z}, 202$ and $\mathrm{m} / \mathrm{z} 146$. 
Figure 5.3. Tandem mass spectra of methoxyacetylfentanyl: a) $\mathrm{MS}^{2}$ product ion spectrum of the $[\mathrm{M}+\mathrm{H}]^{+}$molecular ion (30\% NCE); b) $\mathrm{MS}^{3}$ product ion spectrum of the product ion at $\mathrm{m} / z 260$ (30\% NCE) showing the formation of a dominant product ion at $\mathrm{m} / \mathrm{z} 206$; c) $\mathrm{MS}^{3}$ product ion spectrum of the primary product ion at $\mathrm{m} / \mathrm{z}, 232$ (30\% NCE) showing the formation of product ions at $m / z, 204,176,144$, and 132 .

Figure 5.4. Tandem mass spectra of sufentanil-d $\mathrm{d}_{5}$ : a) $\mathrm{MS}^{2}$ product ion spectrum of the $[\mathrm{M}+\mathrm{H}]^{+}$ molecular ion (30\% NCE); b) $\mathrm{MS}^{3}$ product ion spectrum of the product ion at $\mathrm{m} / \mathrm{z} 360(30 \%$ NCE) showing the formation of product ions at $m / z$ 332, 234, 225, 206, 137 and 111, among others; c) $\mathrm{MS}^{3}$ product ion spectrum of the primary product ion at $\mathrm{m} / \mathrm{z} 238$ (30\% NCE) showing the formation of product ions at $\mathrm{m} / z, 206,140$ and 111 ................................................... 145 Figure 5.5. Tandem mass spectra of $\beta$-hydroxythiolfentanyl- $\mathrm{d}_{5}$ : a) $\mathrm{MS}^{2}$ product ion spectrum of the $[\mathrm{M}+\mathrm{H}]^{+}$molecular ion $(30 \% \mathrm{NCE})$; b) $\mathrm{MS}^{3}$ product ion spectrum of the product ion at $\mathrm{m} / \mathrm{z}$ $346(30 \% \mathrm{NCE})$ showing the formation of product ions at $m / z, 286,221,207,192,158$ and 147 , among others; c) $\mathrm{MS}^{3}$ product ion spectrum of the primary product ion at $m / z 250(30 \% \mathrm{NCE})$ showing the formation of product ions at $\mathrm{m} / \mathrm{z} 207$ and $\mathrm{m} / \mathrm{z} 190$, among others.

Figure 5.6. Tandem mass spectrum of para-methoxybutyrylfentanyl collected a $25 \mathrm{eV}$ collision energy, $250 \mathrm{~V}$ fragmentor voltage and $65 \mathrm{~V}$ skimmer voltage. 148

Figure 5.7. Tandem mass spectrum of alfentanil collected with a $25 \mathrm{eV}$ collision energy, $225 \mathrm{~V}$ fragmentor voltage and $65 \mathrm{~V}$ skimmer voltage. 149

Figure 5.8. Tandem mass spectrum of $\alpha$-methylfentanyl collected with a $25 \mathrm{eV}$ collision energy, $250 \mathrm{~V}$ fragmentor voltage and $65 \mathrm{~V}$ skimmer voltage.

Figure 5.9. Tandem mass spectrum of ${ }^{13} \mathrm{C}_{6}$-carfentanil collected with a $25 \mathrm{eV}$ collision energy, $250 \mathrm{~V}$ fragmentor voltage and $65 \mathrm{~V}$ skimmer voltage.

Figure 5.10. Observed primary product ions for FRCs with ESI-MS/MS. The color of an R group indicates that it tends to direct fragmentation down a pathway of the same color. 152

Figure 6.1. Comparison of beam-type CID (top) and in-source CID (middle and bottom) for protonated ortho-methylfentanyl. For all spectra, the skimmer setting was held at $65 \mathrm{~V}$. For beam-type CID, the fragmentor setting was $250 \mathrm{~V}$ with a collision energy of $25 \mathrm{eV}$. For insource CID, the fragmentor settings were $175 \mathrm{~V}$ and $300 \mathrm{~V}$, for b) and c), respectively with the collision energy set to $0 \mathrm{eV}$. 166

Figure 6.2. Comparison of beam-type CID (top) and in-source CID (middle and bottom) for protonated furanylfentanyl. For all spectra, the skimmer setting was held at $65 \mathrm{~V}$. For beam-type CID, the fragmentor setting was $225 \mathrm{~V}$ with a collision energy of $25 \mathrm{eV}$. For in-source CID, the fragmentor settings were $165 \mathrm{~V}$ and $285 \mathrm{~V}$, for b) and c), respectively with the collision energy set to $0 \mathrm{eV}$.

Figure 6.3. Comparison of beam-type CID (top and middle) and in-source CID (bottom) for protonated alfentanil. For all spectra, the skimmer setting was held at $65 \mathrm{~V}$. For beam-type CID, the fragmentor setting was $225 \mathrm{~V}$ with collision energies of $15 \mathrm{eV}$ and $25 \mathrm{eV}$ for panels a) and b), respectively. For in-source CID, the fragmentor setting was $285 \mathrm{~V}$ with a collision energy of $0 \mathrm{eV}$. 
Figure 6.4. Comparison of beam-type CID (top and middle) and in-source CID (bottom)for protonated $\beta$-hydroxythiolfentanyl-d 5 . For all spectra, the skimmer setting was held at $65 \mathrm{~V}$. For beam-type CID, the fragmentor setting was $225 \mathrm{~V}$ with collision energies of $15 \mathrm{eV}$ and $25 \mathrm{eV}$, for a) and b), respectively. For in-source CID, the fragmentor settings was $285 \mathrm{~V}$ with a collision energy of $0 \mathrm{eV}$..... 173

Figure 6.5. Comparison of beam-type CID (top) and in-source CID (middle and bottom) for protonated methcathinone- $\mathrm{d}_{3}$. For all spectra, the skimmer setting was held at $65 \mathrm{~V}$. For beamtype CID, the fragmentor setting was $175 \mathrm{~V}$ with a collision energy of $25 \mathrm{eV}$. For in-source CID, the fragmentor settings were $175 \mathrm{~V}$ and $255 \mathrm{~V}$, for b) and c), respectively with the collision energy set to $0 \mathrm{eV}$. 179

Figure 6.6. Comparison of beam-type CID (top) and in-source CID (middle and bottom) for protonated diethylpropion. For all spectra, the skimmer setting was held at $65 \mathrm{~V}$. For beam-type CID, the fragmentor setting was $225 \mathrm{~V}$ with a collision energy of $25 \mathrm{eV}$. For in-source CID, the fragmentor settings were $225 \mathrm{~V}$ and $285 \mathrm{~V}$, for b) and c), respectively with the collision energy set to $0 \mathrm{eV}$.

Figure 6.7. Comparison of beam-type CID (top and middle) and in-source CID (bottom) for protonated pentylone- $\mathrm{d}_{3}$. For all spectra, the skimmer setting was held at $65 \mathrm{~V}$. For beam-type $\mathrm{CID}$, the fragmentor setting was $175 \mathrm{~V}$ with collision energies of $15 \mathrm{eV}$ and $25 \mathrm{eV}$, for a) and b), respectively. For in-source CID, the fragmentor setting was $255 \mathrm{~V}$ with a collision energy of 0 $\mathrm{eV}$. 185

Figure 6.8. Comparison of beam-type CID (top and middle) and in-source CID (bottom) and) for protonated dibutylone- $\mathrm{d}_{3}$. For all spectra, the skimmer setting was held at $65 \mathrm{~V}$. For beam-type CID, the fragmentor setting was $225 \mathrm{~V}$ with collision energies of $15 \mathrm{eV}$ and $25 \mathrm{eV}$, for a) and b), respectively. For in-source CID, the fragmentor settings was $285 \mathrm{~V}$ with a collision energy of 0 $\mathrm{eV}$ 188 


\section{List of Tables:}

Table 1.1. Cartesian coordinates of each of optimized structures at $m / z, 119$ in Figure 1.1....... 17

Table 1.2. Cartesian coordinates of each of optimized structures at $m / z 133$ in Figure 1.2....... 19

Table 2.1. Protonated precursor and the five most abundant product ions in decreasing order of abundance for 13 of the synthetic cathinones used in this study. Fragment ions are reported for both the LIT and Q-TOF instruments.

Table 5.1. Protonated precursor ion mass-to-charge values and five most abundant product ions in decreasing order of relative abundance for each compound in this study with the LIT and QTOF instruments. 


\section{List of Symbols/Abbreviations:}

${ }^{13} \mathrm{C}-3$,4-MDPV $-{ }^{13} \mathrm{C}-3,4$-methylenedioxypyrovalerone on the carbonyl carbon

${ }^{13} \mathrm{C}$-carbonyl carbon- $\alpha$-PVP $-{ }^{13} \mathrm{C}$ - $\alpha$-pyrrolidinovalerophenone labeled on the carbonyl $(\beta)$ carbon

${ }^{13} \mathrm{C}$-MPHP $-{ }^{13} \mathrm{C}-4$ '-methyl- $\alpha$-pyrrolidinohexanophenone on the carbonyl carbon

${ }^{13} \mathrm{C}$-PV8 - $\alpha$-pyrrolidinoheptanophenone

${ }^{13} \mathrm{C}$ - $\alpha$-carbon- $\alpha$-PVP $-{ }^{13} \mathrm{C}-\alpha$-pyrrolidinovalerophenone labeled on the $\alpha$-carbon

${ }^{13} \mathrm{C}$ - $\alpha$-PPP $-{ }^{13} \mathrm{C}-\alpha$-pyrrolidinopropiophenone on the $\alpha$-carbon

2,3-MDPV - 2,3-methylenedioxypyrovalerone

3,4-MDPBP - 3,4-methylenedioxy- $\alpha$-pyrrolidinobutiophenone

3,4-MDPPP - 3,4-methylenedioxy- $\alpha$-pyrrolidinopropiophenone

3,4-MDPV - 3,4-methylenedioxypyrovalerone

3,4-MDPV- $\mathrm{d}_{8}-3,4$-methylenedioxypyrovalerone- $\mathrm{d}_{8}$ on the pyrrolidine ring

4-ANPP - 4-anilino- $N$-phenethylpiperidine

4-MeO- $\alpha$-PVP - 4-methoxy- $\alpha$-pyrrolidinopentiophenone

CID - Collision-induced dissociation

Da - Dalton

DART - Direct analysis in real time

DART-MS/MS - Direct analysis in real time tandem mass spectrometry

DAT - Dopamine transporter

DEA - Drug Enforcement Administration

DFT - Density functional theory

EI - Electron ionization

EI-MS - Electron ionization mass spectrometry

ESI - Electrospray ionization

FRCs - Fentanyl-related compounds

GC - Gas chromatography

GC-EI-MS - Gas chromatography-electron ionization-mass spectrometry

HESI - Heated-electrospray ionization 
HRMS - High-resolution mass spectrometry

HSS - Hybrid similarity search

IRMPD - Infrared multiple photon dissociation

IT - Ion trap

LC - Liquid chromatography

LC-ESI-MS/MS - Liquid chromatography-electrospray ionization-tandem mass spectrometry

LIT - Linear ion trap

$m / z$ - Mass to charge ratio

MRM - Multiple reaction monitoring

MS/MS - Tandem mass spectrometry

$\mathrm{MS}^{\mathrm{n}}$ - Multi-stage mass spectrometry

NAT - noradrenaline transporter

NCE - Normalized collision energy

NFLIS - National Forensic Laboratory Information System

NIST - National Institute of Standards and Technology

nLC - Nano-liquid chromatography

NPP - $N$-phenethyl-4-piperidone

NPS - Novel psychoactive substance

PV8 - $\alpha$-pyrrolidinoheptanophenone

Q-TOF - Quadrupole time-of-flight

SID - Surface-induced dissociation

TOF - Time-of-flight

$\alpha$-PBP - $\alpha$-pyrrolidinobutiophenone

$\alpha$-PPP - $\alpha$-pyrrolidinopropiophenone

$\alpha$-PVP - $\alpha$-pyrrolidinovalerophenone

$\alpha-P V P-d_{7}-\alpha-$ pyrrolidinovalerophenone- $\mathrm{d}_{7}$ labeled on the alkyl chain

$\alpha$-PVP- $\mathrm{d}_{8}-\alpha$-pyrrolidinovalerophenone- $\mathrm{d}_{8}$ labeled on the pyrrolidine ring $\alpha$-PVP-methyl group - $\alpha$-methyl-pyrrolidinovalerophenone 


\section{Introduction}

The identification of well-characterized seized drugs is performed thousands of times a day in the United States; however, the expanding use of emerging synthetic drugs is creating a growing problem for both toxicological and seized drug analyses. Not only are the effects and side-effects of most emerging synthetic drugs not known, but the clandestine suppliers continually tweak the chemical structures to keep one step ahead of the law. When forensic chemists and toxicologists attempt to identify these emerging synthetic drugs, the compounds of interest are often absent from the labs' mass spectral database of known substances. As a result, the identities of these emerging synthetic drugs may remain a mystery until an expert in mass spectral interpretation can appropriately interpret the analytical results.

Two of the most rapidly growing areas of emerging synthetic drugs are synthetic cathinones and fentanyl analogs. Synthetic cathinones are phenylalkylamine derivatives, closely related to amphetamines that produce stimulant-like pharmacological effects. In comparison, fentanyl and fentanyl analogs are synthetic opioids that have emerged as some of the deadliest compounds in the growing opioid epidemic in the United States. These two classes of emerging synthetic compounds present different, but equally important problems for their structural characterization. If information about the mass spectral interpretation rules and patterns were more-readily available to toxicologists and seized drug analysts, then analysts would be better equipped to elucidate the identity of emerging synthetic drugs.

To enhance our current understanding of the fragmentation behavior of synthetic cathinones and fentanyl analogs, this work demonstrates the use of multi-stage mass spectrometry $\left(\mathrm{MS}^{\mathrm{n}}\right)$, accurate mass measurements with high-resolution mass spectrometry (HRMS), and isotopic labeling for the structural characterization of these two classes of emerging synthetic drugs. $\mathrm{MS}^{\mathrm{n}}$ 
helped determine the relationships between product ions along different fragmentation pathways. Accurate mass measurements with HRMS enabled the determination of the elemental formulas of each ion. Finally, the use of isotopic labeling enabled the tracking of specific regions of the molecule through the different fragmentation pathways and gave insight into the different structures and rearrangement mechanisms.

The deliverables of this research include the identification of conserved fragmentation pathways for synthetic cathinones and fentanyl analogs, proposed mechanisms for the formation of characteristic ions in both protonated tandem mass spectrometry (MS/MS) and electron ionization mass spectrometry (EI-MS), and discussion about how to apply this new understanding to the identification of novel synthetic cathinones and fentanyl analogs. Chapters 1-3 focus on the mass spectral characterization of synthetic cathinones, Chapters 4-5 focus on fentanyl and it's analogs and Chapter 6 combines both synthetic cathinones and fentanyl analogs.

Chapter 1 describes an investigation into the tandem mass spectrometry of the $\alpha$ pyrrolidinophenone class of synthetic cathinones. Specifically, isotope labeling, ion spectroscopy, density functional theory (DFT) calculations, HRMS and $\mathrm{MS}^{\mathrm{n}}$ analysis of a total of $21 \alpha$ pyrrolidinophenones provided insight into the fragmentation mechanisms that lead to the formation of the tropylium ion at $\mathrm{m} / \mathrm{z} 91$ or substituted tropylium ion such as at $\mathrm{m} / \mathrm{z} 135$ for methylenedioxy-substituted synthetic cathinones. The tropylium ion $(\mathrm{m} / \mathrm{z}, 91)$, or substituted derivative thereof, is among the most abundant product ions in the tandem mass spectra of protonated $\alpha$-pyrrolidinophenone synthetic cathinones, yet the mechanism of formation has not been described and remains a mystery. The absence of a mechanism stems from the difficulty in explaining the elemental composition of the tropylium ion $\left(\mathrm{C}_{7} \mathrm{H}_{7}+{ }^{+}\right)$from the carbon-skeleton of the precursors, which possess the elemental composition $\mathrm{C}_{7} \mathrm{H}_{5} \mathrm{O}$ - on the first seven carbon atoms. Most 
existing mechanisms to form the tropylium ion require a methyl or methylene group adjacent to the phenyl moiety. Our work demonstrates proposed fragmentation mechanisms to explain this phenomenon, which are supported through the use of $\mathrm{MS}^{\mathrm{n}}$, HRMS, isotopic labeling, DFT calculations and gas-phase ion spectroscopy, the latter of which were conducted by our collaborators at Northern Illinois University in the US and at Radboud University in the Netherlands. Chapter 1 has been published in Forensic Chemistry.

Chapter 2 describes the characterization of the $\alpha$-pyrrolidinophenone class of synthetic cathinones using gas chromatography-electron ionization-mass spectrometry (GC-EI-MS), electrospray ionization-tandem mass spectrometry (ESI-MS/MS) and direct analysis in real timetandem mass spectrometry (DART-MS/MS). GC-EI-MS and ESI-MS/MS are prominent techniques in the field of seized drug analysis and toxicology, whereas DART-MS/MS is a developing ambient ionization-based technique designed for increased sample throughput. DARTMS/MS is more prominent in federal laboratories as a fast screening technique. The key structural feature of the $\alpha$-pyrrolidinophenone class of synthetic cathinones is the presence of a pyrrolidine ring on the amine nitrogen of the generic structure. Through this work we establish characteristic protonated tandem mass spectrometry fragmentation pathways and propose mechanistic origins of the EI-MS fragmentation observed for this class of synthetic cathinones. We also provide examples of how this knowledge can be applied to the identification of novel $\alpha$-pyrrolidinophenone synthetic cathinones. Chapter 2 has been accepted for publication in the International Journal of Mass Spectrometry.

Chapter 3 is a continuation of the methodologies applied in Chapter 2; however, the focus of Chapter 3 is the $N$-alkylated class of synthetic cathinones. The $N$-alkylated class of synthetic cathinones is characterized by the presence of one or two alkyl side chains on the amine moiety of 
the generic phenylalkylamine structure to make secondary $\left(2^{\circ}\right)$ or tertiary $\left(3^{\circ}\right)$ amines, respectively. The compounds analyzed represent common substitutions to the core $N$-alkylated synthetic cathinone structure, including various numbers and lengths of $N$-alkyl and aromatic substitutions. The main outcomes of this project are the identification of characteristic protonated MS/MS fragmentation pathways and the generation of proposed mechanistic origins for both the observed protonated MS/MS fragmentation and EI-MS fragmentation. For example, we show that $2^{\circ}$ amines typically have a base peak corresponding to the loss of water, whereas $3^{\circ}$ amines favor the formation of alkylphenones. Also, in contrast to $\alpha$-pyrrolidinophenone synthetic cathinones, which rarely form odd-electron product ions, $N$-alkylated synthetic cathinones readily form radical cations from the even-electron $[\mathrm{M}+\mathrm{H}]^{+}$precursor. These results broaden the current knowledge about the fragmentation behavior of $\mathrm{N}$-alkylated synthetic cathinones. Chapter 3 has been accepted for publication in the International Journal of Mass Spectrometry.

Chapters 4-6 cover the second major branch of this work, which focusses on the mass-spectral characterization of fentanyl and fentanyl analogs. Chapter 4 centers on the composition of the product ions observed at nominal $\mathrm{m} / \mathrm{z}, 188$ for fentanyl and its main synthetic precursor, 4-anilino$\mathrm{N}$-phenethylpiperidine (4-ANPP). The significance of the product ion at nominal $\mathrm{m} / \mathrm{z} 188$ is that it is often used for both qualitative and quantitative determinations due to its dominant abundance in the tandem mass spectra of fentanyl and many fentanyl-related compounds (FRCs). We demonstrate the presence of at least three isobaric fentanyl product ions at nominal $\mathrm{m} / \mathrm{z} 188$, including a novel isobaric product ion that forms through a cross-ring cleavage of the piperidine ring. We also confirmed two previously described isobaric product ions and described their mechanisms of formation through the loss of methylketene $\left(\mathrm{C}_{3} \mathrm{H}_{4} \mathrm{O}\right)$ followed by aniline $\left(\mathrm{C}_{6} \mathrm{H}_{7} \mathrm{~N}\right)$. Chapter 4 has been published in Drug Testing and Analysis. 
Chapter 5 is an extension of the work from Chapter 4, wherein a series of FRCs that are substituted at five common regions of substitution to the core fentanyl structure were analyzed. The goal was to broaden our knowledge about the impact of substitutions on the core fentanyl structure to the tandem mass spectra. The regions of substitution include the aniline ring $\left(\mathrm{R}_{1}\right)$, the amide moiety $\left(\mathrm{R}_{2}\right)$, the piperidine ring $\left(\mathrm{R}_{3}\right)$, the $N$-alkyl chain $\left(\mathrm{R}_{4}\right)$ and the cyclic substituent $\left(\mathrm{R}_{5}\right)$, which is typically a phenyl, thiol or tetrazole. The main finding from this project was that, relative to fentanyl, substitutions on $\mathbf{R}_{1}$ and $\mathbf{R}_{2}$ cause simple and predictable changes in the $m / z$ values for peaks that contain substitutions different from fentanyl. For example, a methyl group on the aniline ring simply shifts all peaks that contain the aniline ring by $14 \mathrm{Da}$. In contrast, modifications to $\mathrm{R}_{3}$ or $\mathrm{R}_{4}$ can cause dramatic alterations to the entire tandem mass spectra. For example, a hydroxyl group on the $N$-alkyl chain $\left(\mathrm{R}_{4}\right)$ causes the MS/MS spectrum to be dominated by the neutral loss of water, but the presence of a methyl group favors the formation of the tropylium ion. These trends and mechanisms help inform practitioners about the relationship between the observed MS/MS spectra and substitutions to the core fentanyl structure, and hopefully places analysts in a better position to either defend their current casework or identify novel FRCs in future casework. Chapter 5 has been published in Drug Testing and Analysis.

Chapter 6 describes the comparison between in-source CID and beam-type CID, as performed on a quadrupole time-of-flight (Q-TOF) mass spectrometer, for a series of previously characterized synthetic cathinones and FRCs. As the name implies, in-source CID involves the fragmentation within the differentially pumped region of the ion source and is accomplished without isolation of the precursor ions. For this reason, in-source CID is usually thought of as pseudo-tandem mass spectrometry. In contrast, beam-type CID involves the isolation of precursor ions in one quadrupole and fragmentation of target precursors ion in the collision cell of the mass 
spectrometer. Throughout the literature in-source CID is used to provide structurally informative product ions with single-stage HRMS instruments as a means of pseudo-tandem mass spectrometry. Our work shows that whereas it is possible to generate structurally diagnostic spectra that are typically very similar to beam-type tandem mass spectra, caution should be used when interpreting in-source CID spectra because there is no isolation step to ensure that the observed product ions only originate from a particular precursor. Chapter 6 has been submitted to Rapid Communications in Mass Spectrometry. 


\section{Chapter 1: Identification of novel fragmentation pathways and fragment ion structures in the tandem mass spectra of protonated synthetic cathinones}

Reproduced in part with permission from J.T. Davidson, E.L. Piacentino, Z.J. Sasiene, Y. Abiedalla, J. DeRuiter, C.R. Clark, G. Berden, J. Oomens, V. Ryzhov, G.P. Jackson, Forensic

Chemistry, DOI: 10.1016/j.forc.2020.100245.

\subsection{Introduction}

Synthetic cathinones are members of a larger class of novel psychoactive substances (NPS) commonly referred to as "designer drugs" or "legal highs" [1]. They are phenylalkylamine derivatives, closely related to amphetamines, which produce stimulant-like pharmacological effects. These effects drive the recreational use of synthetic cathinones, which are often marketed as "not for human consumption" or "bath salts" to avoid legislative restrictions [1-4]. Cathinones are analogs of the natural psychoactive chemical cathinone, which is present in the leaves of the Catha edulis plant, commonly known as khat. This plant is native to the Horn of Africa and the Southwest Arabian Peninsula. Traditionally, khat leaves have been chewed for their stimulant-like effects and used in religious ceremonies such as funerals and weddings [1]. As trade routes expanded, knowledge about the stimulant-like properties of khat leaves extended to Europe and the Western world [ $[\underline{5}, \underline{6}]$.

The first synthetic cathinones to appear on the market in the early twentieth century were originally designed for therapeutic purposes, but recreational use has taken over in the last decade $[\underline{6}, 7]$. Synthetic cathinones are sold in the form of white or yellow amorphous or crystalline powder or in capsules. The quantities usually range from $50 \mathrm{mg}$ to $500 \mathrm{mg}$ packages and the price varies between $\$ 25$ - $\$ 50$ per $50 \mathrm{mg}[\underline{4}, \underline{8}, \underline{9}$. Common brand names include Bloom, Blue Silk, Ivory Wave, Purple Wave, and Vanilla Sky [1, 4]. 
Once synthetic cathinones started to flood the market, countries began to enact legislative restrictions. By 2011 several synthetic cathinones were provisionally scheduled under Schedule I of the United States Controlled Substances Act $[7,10]$. However, the regulation of synthetic cathinones is complicated by the sheer diversity of chemical modifications that are continuously adopted to avoid the regulations imposed on existing analogs [1]. Due to the lag in regulations behind the drugs currently available on the market it is imperative to recognize the characteristic fragmentation of synthetic cathinones and understand the fragmentation pathways through which mass spectra are generated.

Four common families of synthetic cathinones can be identified based on the location of substitution to the core synthetic cathinone structure. The first family of synthetic cathinones are analogs that are $N$-alkylated at the amine moiety, some of which contain ring substituents $[1,11]$. These substances were primarily derived for their therapeutic properties, such as antidepressants. A second family of synthetic cathinones is the pyrrolidinophenone-like family, which are characterized by a pyrrolidinyl substitution at the amine moiety [12]. Another family of synthetic cathinones involves methylation at the $\alpha$-carbon adjacent to the amine nitrogen in the generic synthetic cathinone structure. The last family of synthetic cathinones has both the 3,4methylenedioxy ring substitution and the $N$-pyrrolidinyl moiety [7]. One common variation for all families of synthetic cathinones is varying lengths of the alkyl chains branching from the $\alpha$-carbon.

Whereas many laboratories and research articles conduct routine mass spectrometric analysis of synthetic cathinones, the underlying fragmentation mechanisms that lead to the observed fragment ions are rarely described or understood. However, analysts recognize that structural similarities of synthetic cathinones tend to provide mass-spectral similarities, which greatly assists in the interpretation of spectra of novel synthetic cathinones. 
The seized drug community typically employs gas chromatography-electron ionization-mass spectrometry (GC-EI-MS) to identify unknowns whereas the toxicological community often employs liquid chromatography with electrospray ionization and tandem mass spectrometry (LCESI-MS/MS). Due to differences in the ionization mechanisms, EI primarily produces oddelectron ions and ESI primarily produces even-electron ions. The differences in electron parity and energy deposition typically results in major differences in the fragment mass spectra, as has been demonstrated for a variety of synthetic cathinones [13-18]. For example, Sauer et al. [19] and Abiedalla et al. [20] report the absence of ions at $m / z 91$ and $m / z 135$ for $\alpha$-PVP and 3,4-MDPV (see Methods Section for full names) under EI-MS conditions, but Hasegawa et al. [21] and Fornal [16] report the presence of both ions under ESI-MS/MS conditions.

The analysis of synthetic cathinones with ESI-MS/MS reveals the tropylium ion $(\mathrm{m} / \mathrm{z}$ 91) or methylenedioxy analog $(\mathrm{m} / \mathrm{z} 135)$ as one of the most abundant ions in the protonated tandem mass spectra of many synthetic cathinones [22-25]. Despite the importance of the tropylium ion or methylenedioxy-analog ion in the product ion spectra of synthetic cathinones, previous attempts to explain the mechanistic origin of these important diagnostic ions have been inadequate. As examples, in the first report on the fragmentation of protonated PV8, Swortwood et al. do not address the mechanism of formation of the tropylium ion [26]. In a previous work, Ibanez et al. propose an unsupported 'shift' of the carbonyl group to explain the equivalent ion at $\mathrm{m} / \mathrm{z} 135$ for 3,4-MDPV [27]. Similarly, Pozo et al., Fabregat-Safont et al., and Qian et al. propose mechanisms that involve the loss of $\mathrm{CO}$ directly from the aliphatic chain during the fragmentation of synthetic cathinones without the use of isotopic labeling to support these conclusions [28-30]. Our work now supports these proposed losses of CO. 
In all the above cases, the mechanism(s) are either absent, ambiguous or unsupported with experimental data. A similar problem with the existing knowledge of synthetic cathinone fragmentation is exemplified by accurate mass studies involving methcathinone and ethcathinone fragmentation $[31, \underline{32}$. Bijlsma et al. showed an unexpected product ion at $\mathrm{m} / \mathrm{z} 105.0740$, which must have the elemental composition $\mathrm{C}_{8} \mathrm{H}_{9}{ }^{+}$[31]. The ion is unexpected because its occurrence requires multiple rearrangements. However, the article did not describe the use of isotope labeling or $\mathrm{MS}^{\mathrm{n}}$ experiments, so the mechanism of formation of the $\mathrm{C}_{8} \mathrm{H}_{9}{ }^{+}$fragment remains unclear. The observation of both $\mathrm{m} / \mathrm{z}, 105.0334$ and $\mathrm{m} / \mathrm{z} 105.0697$ from ethcathinone also indicates extensive covalent rearrangements that remain unexplained [32].

These articles demonstrate that there is a significant lack of understanding in the fragmentation behavior of synthetic cathinones with ESI-MS/MS. To better defend the observations of existing casework, to better understand the current observations, and to better predict the fragmentation patterns of future synthetic cathinones, this project examines the fragmentation behavior of synthetic cathinones generated via ESI and analyzed with both ion trap (IT) and quadrupole timeof-flight (Q-TOF) mass spectrometers. IT mass spectrometers are typically nominal mass instruments, but they have the capability to perform multiple stages of mass spectrometry. In contrast, Q-TOF mass spectrometers are high-resolution instruments that provide accurate mass measurements. The combination of multi-stage mass spectrometry $\left(\mathrm{MS}^{\mathrm{n}}\right)$, accurate mass measurements with high-resolution mass spectrometry (HRMS), isotopic labeling and infrared ion spectroscopy allows for the confirmation of intermediate product ions along the proposed fragmentation pathways and provides support for our proposed mechanisms. The identification of a novel fragmentation pathway(s) for the generation of the tropylium ion or methylenedioxy- 
analog ion provides a more coherent framework of understanding for the identification of future synthetic cathinone analogs.

\subsection{Methods}

\subsubsection{Sample Preparation}

This study involved the analysis of 11 synthetic cathinones that were purchased through Cayman Chemical (Ann Arbor, MI, USA) and 11 isotopically labeled or non-commercially available synthetic cathinones synthesized in-house at Auburn University. The synthetic cathinones purchased through Cayman Chemical were: $\alpha$-pyrrolidinopropiophenone $(\alpha$-PPP), $\alpha$ pyrrolidinobutiophenone $\quad(\alpha-\mathrm{PBP}), \quad \alpha$-pyrrolidinovalerophenone $\quad(\alpha$-PVP), $\quad \alpha-$ pyrrolidinoheptanophenone (PV8), 4-methoxy- $\alpha$-pyrrolidinopentiophenone (4-MeO- $\alpha$-PVP), 3',4'-trimethylene- $\alpha$-pyrrolidinovalerophenone, 3,4-methylenedioxy- $\alpha$-pyrrolidinopropiophenone (3,4-MDPPP), 3,4-methylenedioxy- $\alpha$-pyrrolidinobutiophenone $\quad$ (3,4-MDPBP), 3,4methylenedioxypyrovalerone (3,4-MDPV), 3,4-methylenedioxypyrovalerone- $\mathrm{d}_{8} \quad$ on $\quad$ the pyrrolidine ring (3,4-MDPV- $\left.\mathrm{d}_{8}\right)$, and 2,3-methylenedioxypyrovalerone (2,3-MDPV). The synthetic cathinone samples synthesized at Auburn University were: ${ }^{13} \mathrm{C}-\alpha-$ pyrrolidinovalerophenone labeled on the carbonyl carbon $\left({ }^{13} \mathrm{C}\right.$-carbonyl carbon- $\left.\alpha-\mathrm{PVP}\right),{ }^{13} \mathrm{C}-\alpha-$ pyrrolidinovalerophenone labeled on the $\alpha$-carbon $\quad\left({ }^{13} \mathrm{C}-\alpha\right.$-carbon- $\left.\alpha-\mathrm{PVP}\right), \quad{ }^{18} \mathrm{O}-\alpha-$ pyrrolidinovalerophenone $\left({ }^{18} \mathrm{O}-\alpha\right.$-PVP), $\alpha$-pyrrolidinovalerophenone- $\mathrm{d}_{7}$ labeled on the alkyl chain ( $\alpha$-PVP- $\left.\mathrm{d}_{7}\right), \alpha$-pyrrolidinovalerophenone- $\mathrm{d}_{8}$ labeled on the pyrrolidine ring $\left(\alpha-\mathrm{PVP}-\mathrm{d}_{8}\right), \alpha-$ methylpyrrolidinovalerophenone ( $\alpha$-PVP-methyl group), ${ }^{13} \mathrm{C}$ - $\alpha$-pyrrolidinoheptanophenone labeled on the carbonyl carbon $\left({ }^{13} \mathrm{C}\right.$-PV8), ${ }^{13} \mathrm{C}$ - $\alpha$-pyrrolidinopropiophenone on the $\alpha$-carbon $\left({ }^{13} \mathrm{C}\right.$ - $\alpha$-PPP $)$, ${ }^{13} \mathrm{C}-4$ '-methyl- $\alpha$-pyrrolidinohexanophenone on the carbonyl carbon $\left({ }^{13} \mathrm{C}-\mathrm{MPHP}\right),{ }^{13} \mathrm{C}-3,4-$ methylenedioxypyrovalerone on the carbonyl carbon $\left({ }^{13} \mathrm{C}-3,4-\mathrm{MDPV}\right)$, and ${ }^{13} \mathrm{C}-\mathrm{Naphyrone}$ on the 
carbonyl carbon. Before the synthetic samples were shipped to West Virginia University, a full characterization using NMR and GC-EI-MS was performed at Auburn University to confirm the correct labeling in acceptable purity. All samples were analyzed at a concentration of approximately $100 \mathrm{ppm}$. The non-deuterated samples were dissolved in a solution of 49\% HPLC grade methanol, $49 \%$ distilled water and $2 \%$ acetic acid. Deuterated samples were dissolved in HPLC grade methanol only to prevent back exchange. The HPLC-grade methanol was supplied by Fisher Scientific (Palo Alto, CA, USA) and the acetic acid was supplied by Acros Organics (Palo Alto, CA, USA).

\subsubsection{Instrumentation}

\subsubsection{Velos Pro Linear Ion Trap}

A Thermo Scientific Velos Pro Linear Ion Trap (LIT) mass spectrometer was operated with heated-electrospray ionization (HESI). The HESI source was operated at $50{ }^{\circ} \mathrm{C}$ with a spray voltage of $4,000 \mathrm{~V}$. The nitrogen sheath gas was operated at 8 arbitrary units with a nitrogen auxiliary gas flow of 5 arbitrary units. The mass spectrometer capillary temperature was $275^{\circ} \mathrm{C}$. The scan range and normalized collision energy (NCE) were different for each compound and are labeled with each mass spectrum. Ultra-pure helium was used as the bath gas purchased through Matheson TRIGAS (Fairmont, WV, USA).

\subsubsection{Agilent Technologies 6538 UHD Accurate-Mass Quadrupole Time-of-Flight (Q-TOF)}

An Agilent Technologies 6538 UHD Accurate-Mass Quadrupole Time-of-flight (Q-TOF) mass spectrometer was operated with a dual ESI source at a spray voltage of 3,500 V. The nitrogen gas was set to $300{ }^{\circ} \mathrm{C}$ with a drying gas flow of $5 \mathrm{~L} / \mathrm{min}$ and a nebulizer flow of $30 \mathrm{psig}$. The MS fragmentor and skimmer voltages were operated at $225 \mathrm{~V}$ and $65 \mathrm{~V}$, respectively. The scan range and collision energy were different for each compound and are labeled in each mass spectrum. An 
isolation width of 1.3 Da was used for all samples. Ultra-pure nitrogen was used for the collision gas purchased through Matheson TRIGAS (Fairmont, WV, USA).

\subsubsection{Ion Spectroscopy}

Gas-phase infrared ion spectroscopy experiments were performed at the FELIX laboratory in Nijmegen, Netherlands using an electrospray ionization source on a Bruker Amazon ion trap mass spectrometer, modified to provide optical access to the trapped ions [33]. The flow rate of the $\alpha$ PVP sample to the source was $120 \mu \mathrm{L} / \mathrm{hr}$ with a spray voltage of $-4500 \mathrm{~V}$ and $\mathrm{N}_{2}$ nebulizer gas was used. Precursor ions at $\mathrm{m} / z 119$ and $\mathrm{m} / z 133$ were generated through ESI-MS/MS of $\alpha$-PVP (fragmentation of $[\mathrm{M}+\mathrm{H}]^{+}$at $\mathrm{m} / \mathrm{z} 232$ ), isolated in the ion trap and irradiated with 10 infrared laser pulses from the free electron laser (FEL) (repetition rate $10 \mathrm{~Hz}$, pulse energies between 80 and 200 $\mathrm{mJ})$, which was tuned over the frequency range $1000-1850 \mathrm{~cm}^{-1}$. The recorded mass spectra were used to determine the infrared multiple photon dissociation (IRMPD) yield at each wavelength, which is defined as the ratio of the summed product ion intensities divided by the total ion intensity. After measuring the intensities of the precursor and fragment ions at a given wavelength of irradiation, the IR frequency was changed in steps of $3 \mathrm{~cm}^{-1}$. For each IR frequency, new packets of ions were loaded into the ion trap and irradiated. The intensities of the precursor and product ions were the average of five replicate mass spectra per IR step. The whole process continued across the fingerprint spectral region (1000- $1850 \mathrm{~cm}^{-1}$ ). IRMPD spectra were linearly corrected for variations in laser power as a function of IR frequency. The experimental gas-phase IRMPD spectra could then be compared to the density functional theory (DFT) calculated spectra.

The lowest-energy geometry was calculated at the B3LYP/6-311+G(d,p) level of theory using Gaussian 09 [34] for several isomeric structures of each of the two intermediate product ions at $\mathrm{m} / \mathrm{z} 119$ (Figure 1.1) and m/z 133 (Figure 1.2). The Cartesian coordinates for the optimized 
isomeric structures for the product ions at $\mathrm{m} / \mathrm{z}, 119$ and $\mathrm{m} / \mathrm{z}, 133$ are presented in Table $\mathbf{1 . 1}$ and Table 1.2, respectively. The vibrational frequencies calculated at this level of theory provided thermodynamic corrections to the raw energies and the theoretical vibrational spectra of each of the species. Comparison of the calculated and the IRMPD spectra, along with the relative energies between isomers, provided justification for the assignment of the most probable isomer of each of the species. 
$119 a$
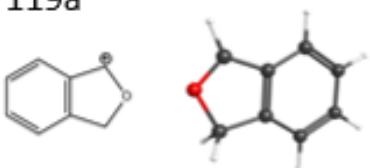

0.0
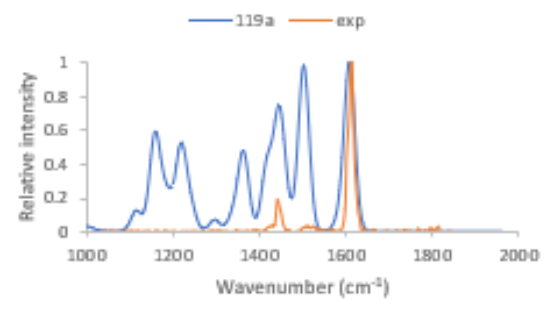

$119 \mathrm{c}$
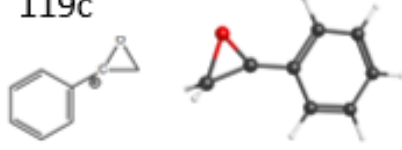

$-119 c-\exp$
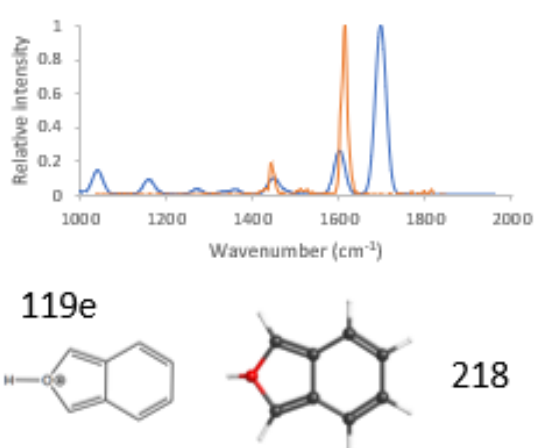

$-119 e-\exp$

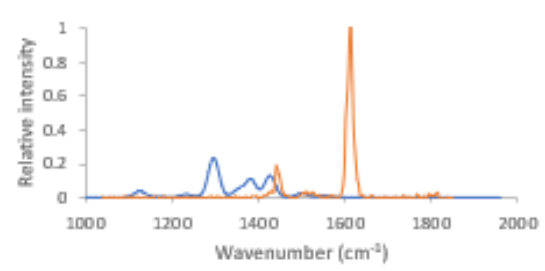

218

$119 \mathrm{~g}$
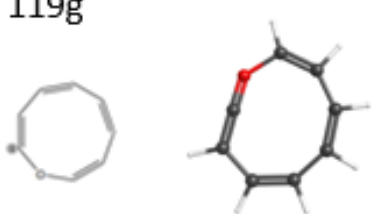

$119 \mathrm{~b}$
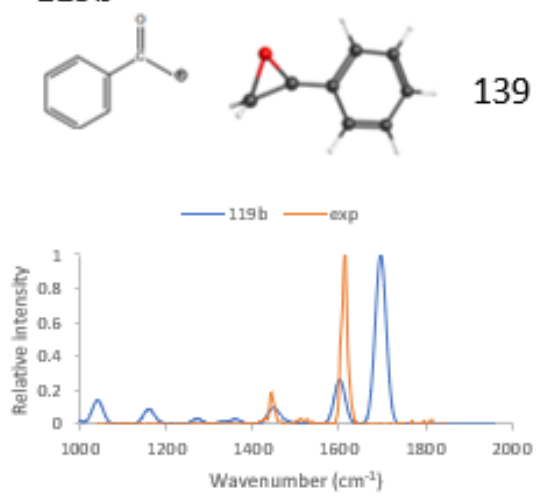

$119 d$
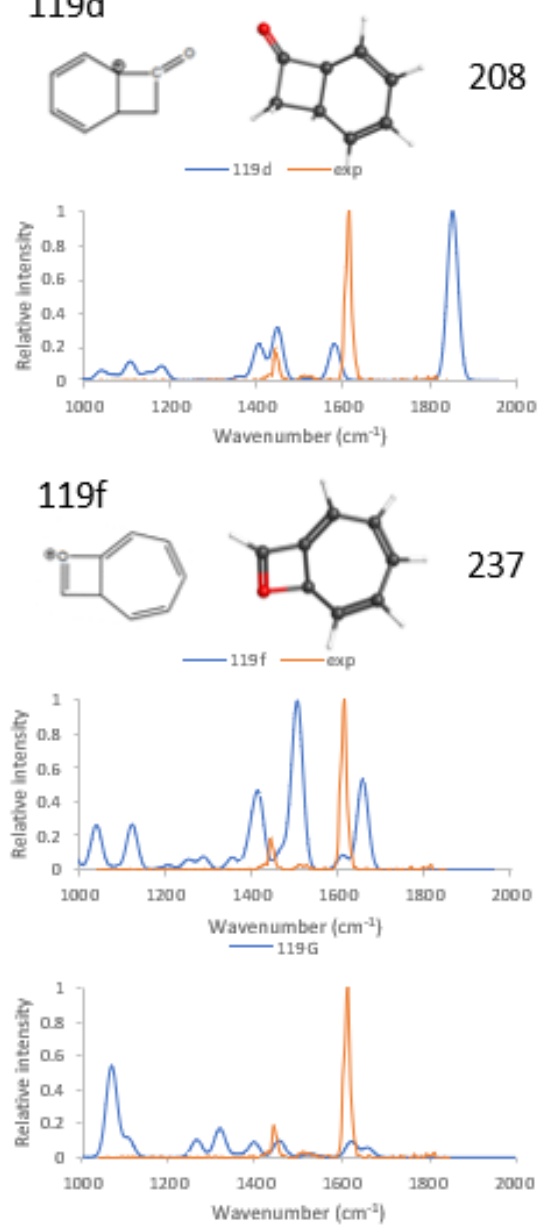

Figure 1.1. Optimized geometries of seven possible isomeric structures of the fragment at $\mathrm{m} / \mathrm{z}$ 119. Relative energies and theoretical IR spectra are calculated at the B3LYP/6-311+G(d,p) level of theory. Energies are reported in $\mathrm{kJ} / \mathrm{mol}$ relative to structure 119a. Calculated IR (blue) are overlapped with the experimental spectrum (orange) of the $m / z 119$ fragment. 
$133 a$

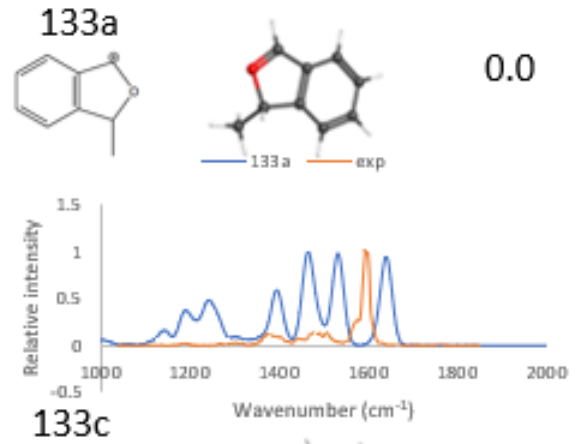

$133 c$
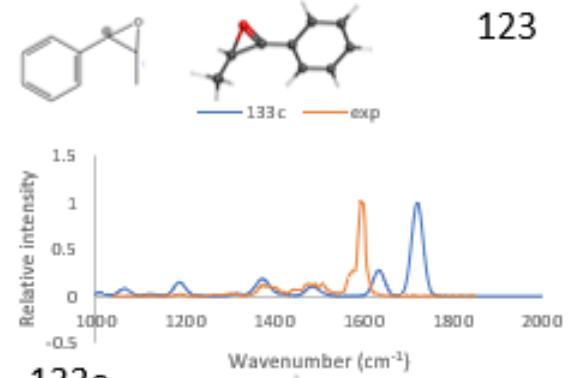

$133 \mathrm{e}$
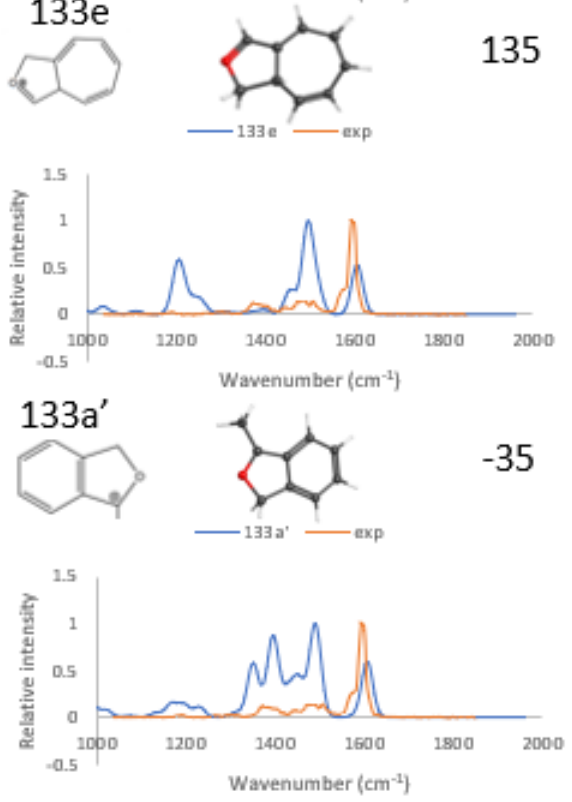

$133 \mathrm{~g}$

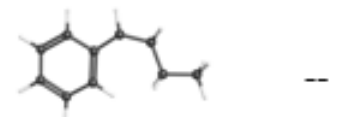

$133 b$
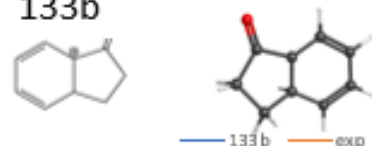

116

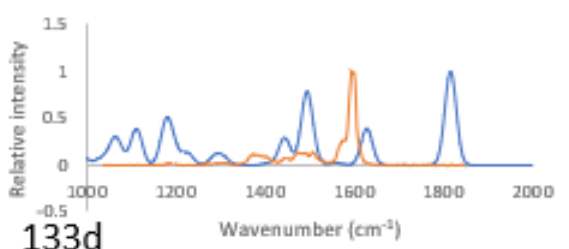

$133 d$
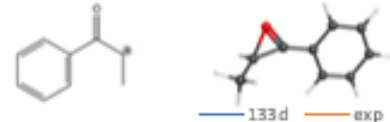

123

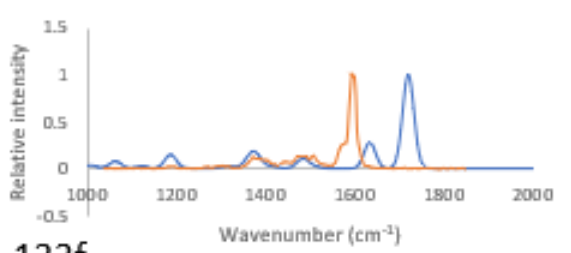

$133 f$

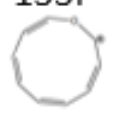

479

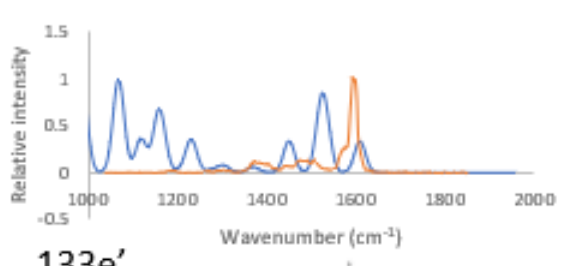

$133 e^{\prime}$
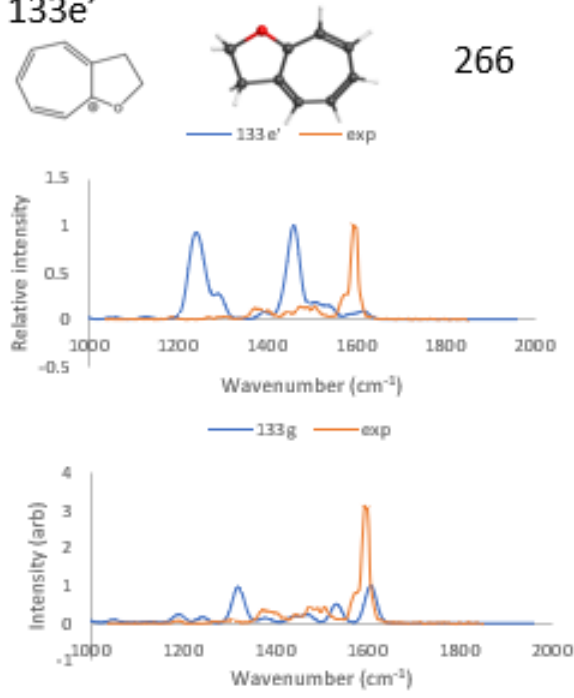

Figure 1.2. Optimized geometries of nine possible isomeric structures of the fragment at $\mathrm{m} / \mathrm{z}$ 133. Relative energies and theoretical IR spectra are calculated at the B3LYP/6-311+G(d,p) level of theory. Energies are reported in $\mathrm{kJ} / \mathrm{mol}$ relative to structure 133a. Calculated IR (blue) are overlapped with the experimental spectrum (orange) of the $\mathrm{m} / \mathrm{z} 133$ fragment. 
Table 1.1. Cartesian coordinates of each of optimized structures at $m / z 119$ in Figure 1.1. 


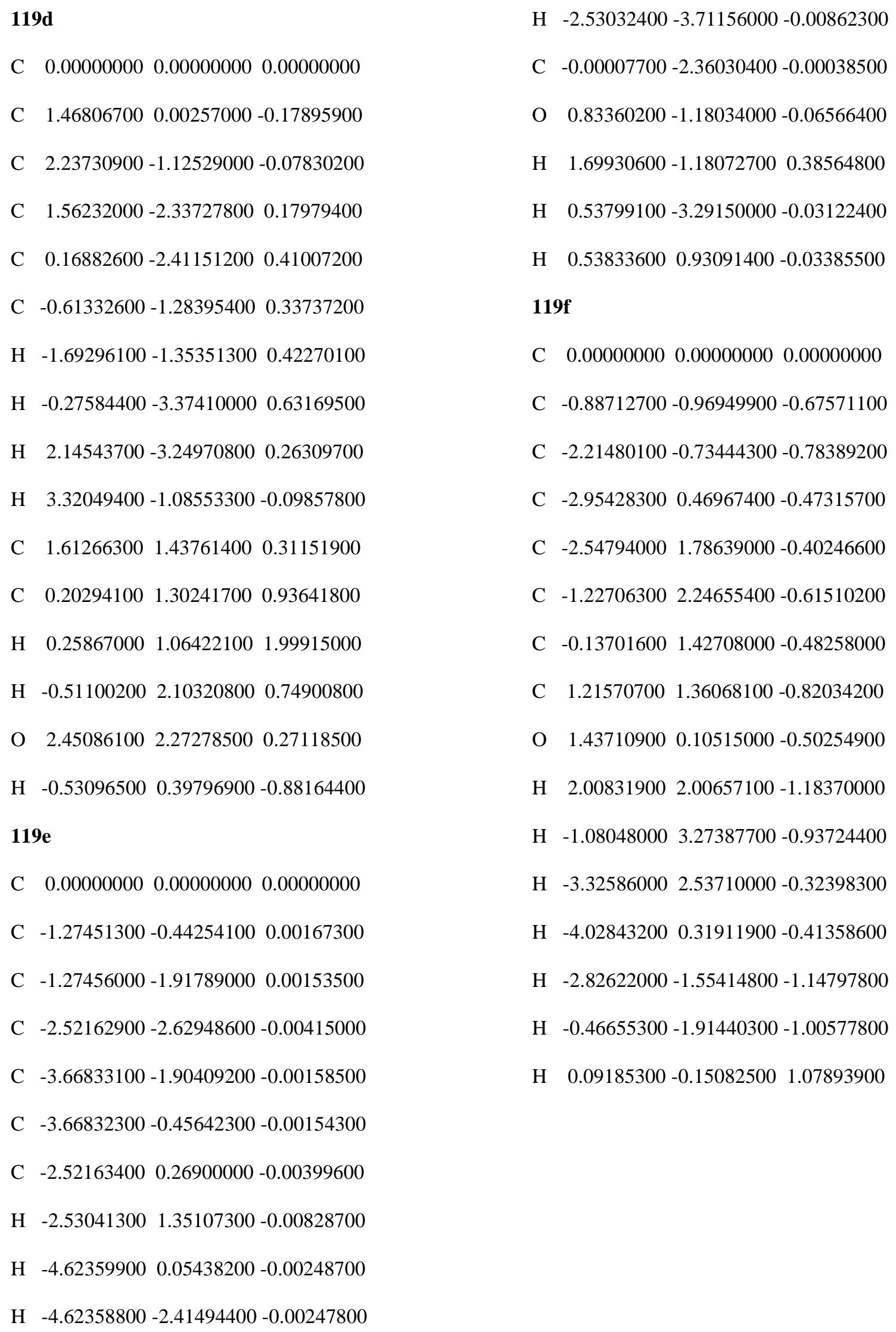


Table 1.2. Cartesian coordinates of each of optimized structures at $m / z 133$ in Figure 1.2. 


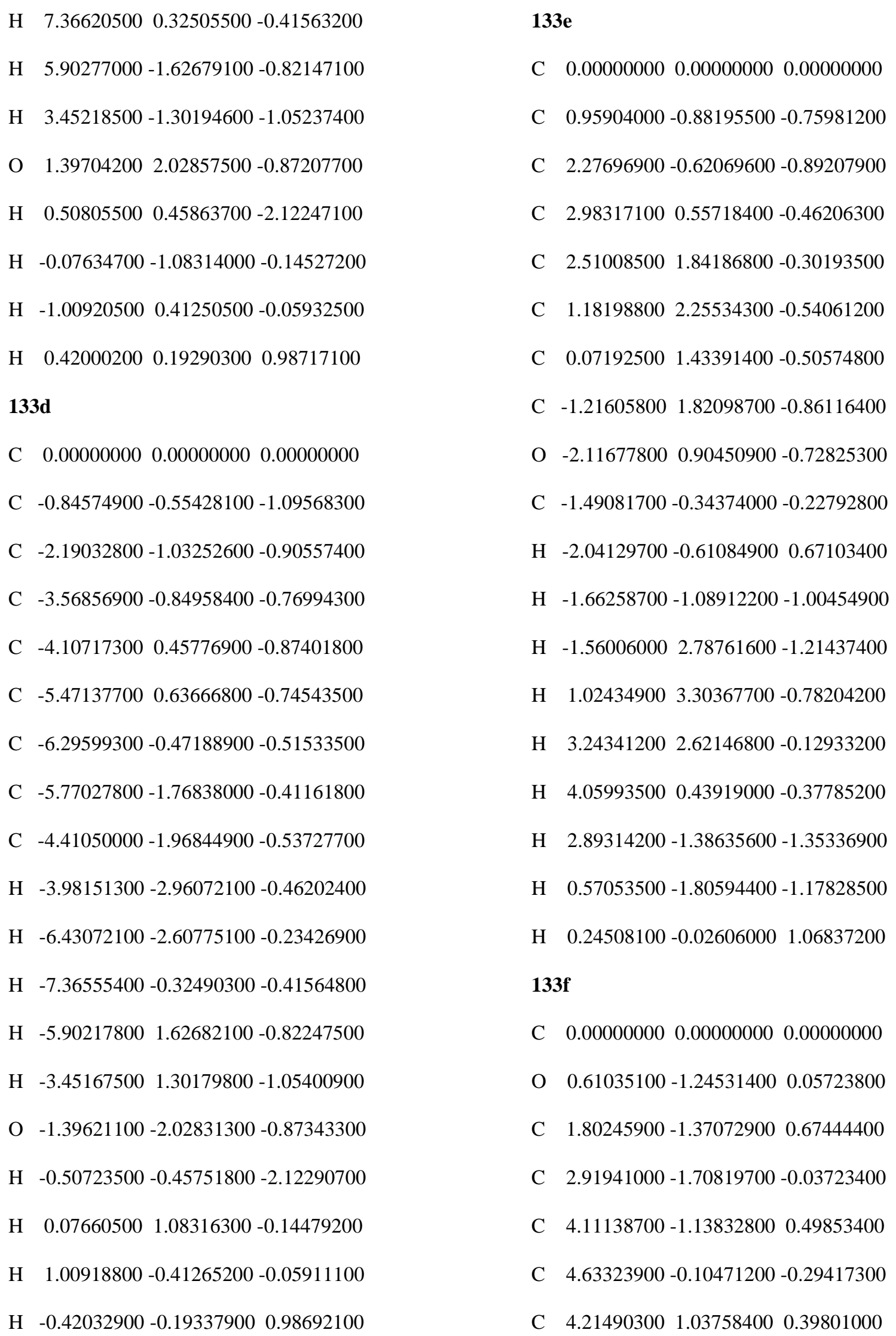

\section{3e}

C $\quad 0.00000000 \quad 0.00000000 \quad 0.00000000$

C $\quad 0.95904000-0.88195500-0.75981200$

C $2.27696900-0.62069600-0.89207900$

C $2.98317100 \quad 0.55718400-0.46206300$

C $\quad 2.51008500 \quad 1.84186800-0.30193500$

C $\quad 1.181988002 .25534300-0.54061200$

C $\quad 0.07192500 \quad 1.43391400-0.50574800$

C $\quad-1.21605800 \quad 1.82098700-0.86116400$

O $\quad-2.11677800 \quad 0.90450900-0.72825300$

C $-1.49081700-0.34374000-0.22792800$

H $\quad-2.04129700-0.61084900 \quad 0.67103400$

H $\quad-1.66258700-1.08912200-1.00454900$

H $\quad-1.56006000 \quad 2.78761600-1.21437400$

H $\quad 1.024349003 .30367700-0.78204200$

H $3.243412002 .62146800-0.12933200$

H $\quad 4.05993500 \quad 0.43919000-0.37785200$

H $2.89314200-1.38635600-1.35336900$

H $\quad 0.57053500-1.80594400-1.17828500$

H $\quad 0.24508100-0.02606000 \quad 1.06837200$

$133 f$

C $\quad 0.00000000 \quad 0.00000000 \quad 0.00000000$

O $\quad 0.61035100-1.24531400 \quad 0.05723800$

C $\quad 1.80245900-1.37072900 \quad 0.67444400$

C $2.91941000-1.70819700-0.03723400$

C $\quad 4.11138700-1.13832800 \quad 0.49853400$

C $4.63323900-0.10471200-0.29417300$

C $\quad 4.21490300 \quad 1.03758400 \quad 0.39801000$ 


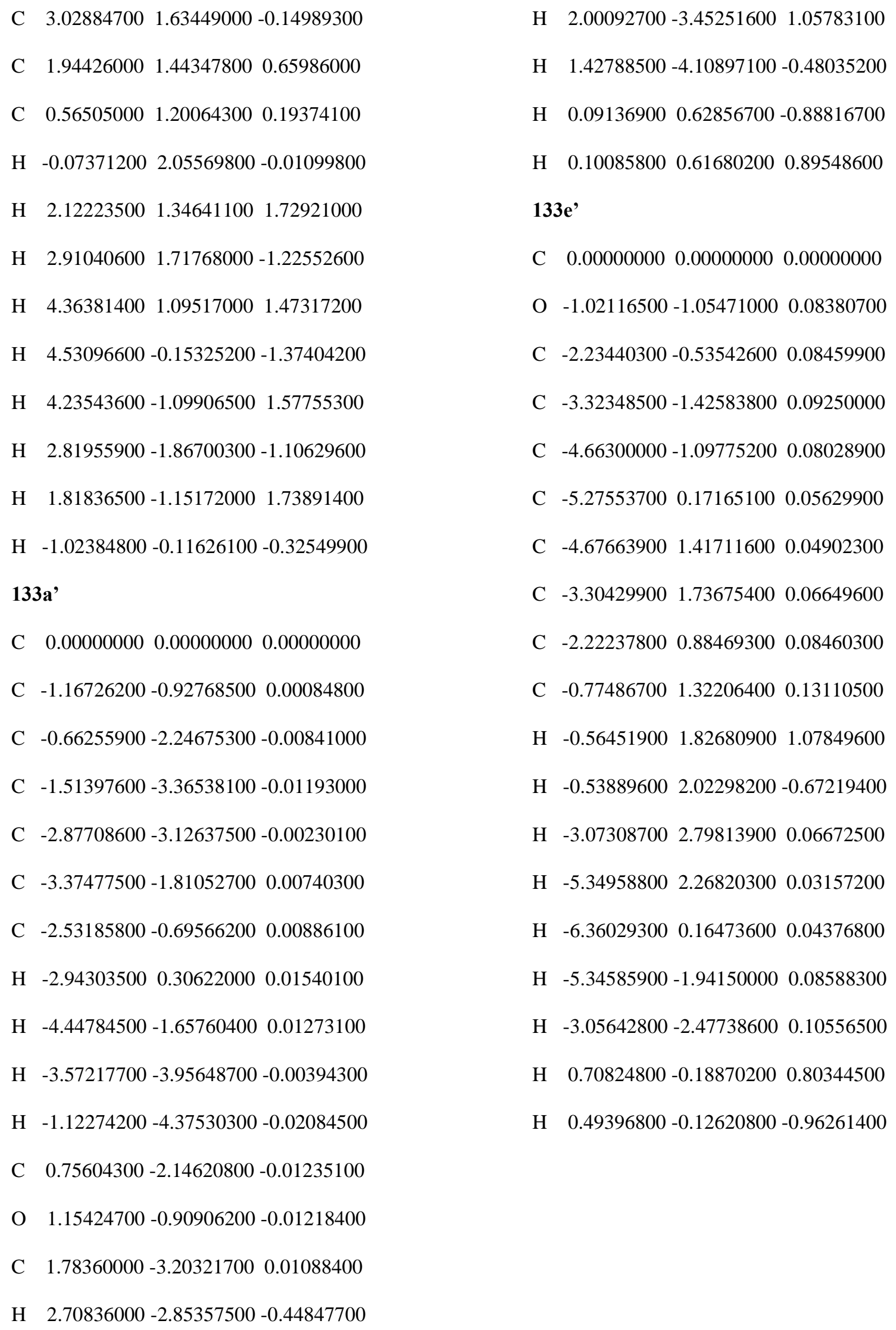




\section{3a}

$\begin{array}{llll}\text { C } & 0.00000000 & 0.00000000 & 0.00000000\end{array}$

C $-1.48676700-0.024759000 .17268700$

C $\quad-1.93158700 \quad 1.30027100 \quad 0.40346300$

C $\quad-3.28903600 \quad 1.61348700 \quad 0.60698100$

C $\quad-4.18900800 \quad 0.56420400 \quad 0.57233400$

C $\quad-3.74574800-0.75222000 \quad 0.34275100$

C $-2.39695400-1.06563100 \quad 0.14034500$

H $-2.09262200-2.08993000-0.03593300$

H $\quad-4.47737900-1.55194500 \quad 0.32254400$

$\begin{array}{llll}\mathrm{H} & -5.24500100 & 0.74963600 & 0.72275300\end{array}$

H $\quad-3.61245600 \quad 2.63230000 \quad 0.78256000$

C $\quad-0.77942500 \quad 2.10720900 \quad 0.37954900$

$\begin{array}{llll}\mathrm{O} & 0.30899600 & 1.45736000 & 0.16571600\end{array}$

H $\quad-0.68629200 \quad 3.18075200 \quad 0.51326500$

C $\quad 0.84098400-0.79093200 \quad 0.98833700$

H $\quad 1.90090200-0.59959600 \quad 0.81933400$

H $\quad 0.58271300-0.538171002 .01775100$

H $\quad 0.65490700-1.85586700 \quad 0.83202800$

H $\quad 0.29934100-0.20733900-1.03180400$

\section{3b}

C $\quad 0.00000000 \quad 0.00000000 \quad 0.00000000$

C $\quad 0.38282900-1.40359100 \quad 0.16356600$

C $-0.53485400-2.40542900 \quad 0.33112500$

C $-1.89015200-2.03541300 \quad 0.44457600$

C $-2.32528400-0.69254700 \quad 0.39329300$

C $\quad-1.41640200 \quad 0.31908600 \quad 0.20042900$

H $\quad-1.74136600 \quad 1.35114200 \quad 0.11261100$
H $\quad-3.37934300-0.47181300 \quad 0.50921900$

H $\quad-2.63167800-2.81185500 \quad 0.60740200$

H $\quad-0.22224600-3.43774000 \quad 0.44219300$

C $\quad 1.89194100-1.49461400 \quad 0.26781200$

C $\quad 2.41592700-0.06437700 \quad 0.35122700$

C $\quad \begin{array}{llll}1.18379400 & 0.81860300 & 0.63759900\end{array}$

$\mathrm{H} \quad 1.25354400 \quad 1.81851100 \quad 0.21308700$

H $\quad 1.00579100 \quad 0.911196001 .71032600$

H $\quad 2.87952800 \quad 0.18515500-0.61035700$

H $\quad 3.19622700 \quad 0.014201001 .10932900$

O $\quad 2.50176000-2.52781800 \quad 0.30314500$

H $\quad 0.11237300 \quad 0.18513700-1.09997700$

\section{3c}

C $\quad 0.00000000 \quad 0.00000000 \quad 0.00000000$

C $\quad 0.84621700 \quad 0.55467600-1.09505700$

C $\quad 2.19084600 \quad 1.03253000-0.90432200$

C $3.56907200 \quad 0.84950400-0.76875500$

C $\quad 4.10769700-0.45784400-0.87270000$

C $\quad 5.47194300-0.63664100-0.74450700$

C $\quad 6.29659900 \quad 0.47199500-0.51493500$

C $\quad 5.77087400 \quad 1.76848200-0.41125000$

C $4.411049001 .96844100-0.53645000$

H $3.981990002 .96068800-0.46127100$

H $\quad 6.431344002 .60791400-0.23428800$

H $7.36620500 \quad 0.32505500-0.41563200$

H $\quad 5.90277000-1.62679100-0.82147100$

H $\quad 3.45218500-1.30194600-1.05237400$

O $\quad 1.397042002 .02857500-0.87207700$ 

H $\quad 0.50805500 \quad 0.45863700-2.12247100$
C $\quad 2.98317100 \quad 0.55718400-0.46206300$
H $\quad-0.07634700-1.08314000-0.14527200$
C $\quad 2.51008500 \quad 1.84186800-0.30193500$
H $\quad-1.00920500 \quad 0.41250500-0.05932500$
C $\quad 1.18198800 \quad 2.25534300-0.54061200$
H $\quad 0.42000200 \quad 0.19290300 \quad 0.98717100$
C $\quad 0.07192500 \quad 1.43391400-0.50574800$
133d
C $\quad-1.21605800 \quad 1.82098700-0.86116400$
C $\quad 0.00000000 \quad 0.00000000 \quad 0.00000000$
O $\quad-2.11677800 \quad 0.90450900-0.72825300$
C $-0.84574900-0.55428100-1.09568300$
C $\quad-1.49081700-0.34374000-0.22792800$
C $-2.19032800-1.03252600-0.90557400$
H $\quad-2.04129700-0.61084900 \quad 0.67103400$
C $\quad-3.56856900-0.84958400-0.76994300$
H $\quad-1.66258700-1.08912200-1.00454900$
C $\quad-4.10717300 \quad 0.45776900-0.87401800$
H $\quad-1.56006000 \quad 2.78761600-1.21437400$
C $\quad-5.47137700 \quad 0.63666800-0.74543500$
H $\quad 1.024349003 .30367700-0.78204200$
C $-6.29599300-0.47188900-0.51533500$
H $\quad 3.24341200 \quad 2.62146800-0.12933200$
C $\quad-5.77027800-1.76838000-0.41161800$
H $\quad 4.05993500 \quad 0.43919000-0.37785200$
C $-4.41050000-1.96844900-0.53727700$
H $2.89314200-1.38635600-1.35336900$
H $\quad-3.98151300-2.96072100-0.46202400$
H $\quad 0.57053500-1.80594400-1.17828500$
H $\quad-6.43072100-2.60775100-0.23426900$
H $\quad 0.24508100-0.02606000 \quad 1.06837200$
H $\quad-7.36555400-0.32490300-0.41564800$
133f
H $\quad-5.90217800 \quad 1.62682100-0.82247500$
C $\quad 0.00000000 \quad 0.00000000 \quad 0.00000000$
H $\quad-3.45167500 \quad 1.30179800-1.05400900$
O $\quad 0.61035100-1.24531400 \quad 0.05723800$
O $-1.39621100-2.02831300-0.87343300$
C $\quad 1.80245900-1.37072900 \quad 0.67444400$
H $\quad-0.50723500-0.45751800-2.12290700$
C $2.91941000-1.70819700-0.03723400$
H $\quad 0.07660500 \quad 1.08316300-0.14479200$
C $\quad 4.11138700-1.13832800 \quad 0.49853400$
H $\quad 1.00918800-0.41265200-0.05911100$
C $4.63323900-0.10471200-0.29417300$
H $\quad-0.42032900-0.19337900 \quad 0.98692100$
C $\quad 4.21490300 \quad 1.03758400 \quad 0.39801000$

\section{3e}
C $\quad 3.02884700 \quad 1.63449000-0.14989300$
C $\quad 0.00000000 \quad 0.00000000 \quad 0.00000000$
C $\quad 1.94426000 \quad 1.44347800 \quad 0.65986000$
C $0.95904000-0.88195500-0.75981200$
C $\quad 0.56505000 \quad 1.20064300 \quad 0.19374100$
C $\quad 2.27696900-0.62069600-0.89207900$
H $\quad-0.07371200 \quad 2.05569800-0.01099800$ 


\begin{tabular}{|c|c|c|c|c|c|}
\hline $\mathrm{H}$ & $2.12223500 \quad 1.34641100 \quad 1.72921000$ & $\mathrm{H}$ & 2.00092700 & -3.45251600 & 1.05783100 \\
\hline H & $2.91040600 \quad 1.71768000-1.22552600$ & $\mathrm{H}$ & 1.42788500 & -4.10897100 & -0.48035200 \\
\hline $\mathrm{H}$ & $\begin{array}{llll}4.36381400 & 1.09517000 & 1.47317200\end{array}$ & $\mathrm{H}$ & 0.09136900 & 0.62856700 & -0.88816700 \\
\hline H & $4.53096600-0.15325200-1.37404200$ & $\mathrm{H}$ & 0.10085800 & 0.61680200 & 0.89548600 \\
\hline H & $4.23543600-1.099065001 .57755300$ & \multicolumn{4}{|c|}{$133 \mathrm{e}^{\prime}$} \\
\hline H & $2.81955900-1.86700300-1.10629600$ & $\mathrm{C}$ & 0.00000000 & 0.00000000 & 0.00000000 \\
\hline H & $1.81836500-1.15172000 \quad 1.73891400$ & $\mathrm{O}$ & -1.02116500 & -1.05471000 & 0.08380700 \\
\hline $\mathrm{H}$ & $-1.02384800-0.11626100-0.32549900$ & $\mathrm{C}$ & -2.23440300 & -0.53542600 & 0.08459900 \\
\hline & 3a' & $\mathrm{C}$ & -3.32348500 & -1.42583800 & 0.09250000 \\
\hline C & $0.00000000 \quad 0.00000000 \quad 0.00000000$ & $\mathrm{C}$ & -4.66300000 & -1.09775200 & 0.08028900 \\
\hline E & 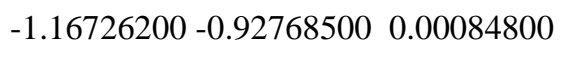 & $\mathrm{C}$ & -5.27553700 & 0.17165100 & 0.05629900 \\
\hline c & $-0.66255900-2.24675300-0.00841000$ & $\mathrm{C}$ & -4.67663900 & 1.41711600 & 0.04902300 \\
\hline F & $-1.51397600-3.36538100-0.01193000$ & $\mathrm{C}$ & -3.30429900 & 1.73675400 & 0.06649600 \\
\hline $\mathrm{C}$ & $-2.87708600-3.12637500-0.00230100$ & $\mathrm{C}$ & -2.22237800 & 0.88469300 & 0.08460300 \\
\hline $\mathrm{C}$ & $-3.37477500-1.810527000 .00740300$ & $\mathrm{C}$ & -0.77486700 & 1.32206400 & 0.13110500 \\
\hline c & 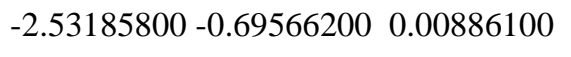 & $\mathrm{H}$ & -0.56451900 & 1.82680900 & 1.07849600 \\
\hline $\mathrm{H}$ & $\begin{array}{llll}-2.94303500 & 0.30622000 & 0.01540100\end{array}$ & $\mathrm{H}$ & -0.53889600 & 2.02298200 & -0.67219400 \\
\hline $\mathrm{H}$ & $-4.44784500-1.657604000 .01273100$ & $\mathrm{H}$ & -3.07308700 & 2.79813900 & 0.06672500 \\
\hline $\mathrm{H}$ & $-3.57217700-3.95648700-0.00394300$ & $\mathrm{H}$ & -5.34958800 & 2.26820300 & 0.03157200 \\
\hline $\mathrm{H}$ & $-1.12274200-4.37530300-0.02084500$ & $\mathrm{H}$ & -6.36029300 & 0.16473600 & 0.04376800 \\
\hline C & $0.75604300-2.14620800-0.01235100$ & $\mathrm{H}$ & -5.34585900 & -1.94150000 & 0.08588300 \\
\hline o & $1.15424700-0.90906200-0.01218400$ & $\mathrm{H}$ & -3.05642800 & -2.47738600 & 0.10556500 \\
\hline C & $1.78360000-3.20321700 \quad 0.01088400$ & $\mathrm{H}$ & 0.70824800 & -0.18870200 & 0.80344500 \\
\hline & $2.70836000-2.85357500-0.44847700$ & $\mathrm{H}$ & 0.49396800 & -0.12620800 & -0.96261400 \\
\hline
\end{tabular}




\subsubsection{Data Analysis}

Xcalibur 2.0.0.48 software was used for the data analysis on the Velos Pro and Mass Hunter Qualitative Analysis B.05.00 was used for the Agilent Q-TOF data analysis. Microsoft Excel version 14 (Microsoft, Redmond, WA, USA) and ChemDraw 16.0 (PerkinElmer, Waltham, MA, USA) were used for mass spectral plots and mass spectral fragmentation mechanisms, respectively.

\subsubsection{Mass Spectral Interpretation and Mechanisms}

The proposed fragmentation mechanisms in the following section are based on $\mathrm{MS}^{\mathrm{n}}$ analyses, rational electron pushing mechanisms and the expected lowest energy pathways [35]. Whereas the identification of the exact hydrogen(s) in a specific rearrangement is not always possible in this study, deuterium labeling was often able to exclude the involvement of certain hydrogen atoms. The use of $\mathrm{MS}^{\mathrm{n}}$ permits the structural determination of all the intermediates along a fragmentation pathway, so even when the exact structure of an intermediate is not known, it is still possible to generate a deeper understanding of the precursors and products of a certain intermediate than the present status. Odd-electron product ions formed from even-electron (i.e. protonated) precursor ions of synthetic cathinones have been reported before by Fornal [36, 37], but none of the $\alpha-$ pyrrolidinophenone synthetic cathinones analyzed in this study provided a significant abundance of odd-electron product ions, which is consistent with previous literature [36].

\subsection{Results and Discussion}

\subsubsection{HESI-Velos Pro $M^{n}$}

Since the seminal publication by Rylander et al. in 1956 [38], the propensity of aromatic compounds to form energetically favored tropylium fragments has been studied extensively in EIMS spectra. The original article recognized the thermodynamic and tautomeric benefits of 
rearrangement of the benzylium ion $\left(\mathrm{C}_{7} \mathrm{H}_{7}{ }^{+}, m / z\right.$ 91) to the tropylium ion, which is a constitutional isomer. In the 1970s, McLafferty et al. focused on the formation of tropylium ions from a variety of alkyl-substituted benzenes under EI-MS conditions [여, $\underline{40]}$ McLafferty and coworkers showed that whereas benzylium and tropylium ions are often equally favored at threshold fragmentation energies, the tropylium ion is favored by a factor of at least 2:1 at EI energies around $70 \mathrm{eV}[\underline{39}$, 40].

Lifshitz et al. also demonstrated that the tropylium ion is more stable than several other isomers and noticeably lower in energy than the benzylium ion, which explains the preference for the ring expansion of benzylium ions to the tropylium ion structure [41]. More recently, Hayward et al. have described the formation of the tropylium ion with surface-induced dissociation (SID) [42]. Hayward et al. showed that the ring expansion from the benzylium ion to the tropylium ion was exothermic and often involves the incorporation of alkyl substituents. Specifically, the reaction of neutralized benzene with sputtered $\mathrm{C}_{3} \mathrm{H}_{5}{ }^{+}$followed by the loss of ethene was the most likely route for tropylium ion formation [42]. Using a variety of theoretical calculations, several groups have shown that the activation barrier from the benzylium ion to the tropylium ion is in the range of 1.4$3.4 \mathrm{eV}$ and that the tropylium ion is thermodynamically more stable than the benzylium ion by approximately $0.37 \mathrm{eV}$ [43-49]. Although the mechanism(s) from the benzylium ion to tropylium ion is therefore very well documented, mechanisms to form the tropylium ion from aromatic ketones - like alkylphenones and cathinones — have not been adequately described. We therefore conducted various experiments to rationalize the significant rearrangements that are required to produce the tropylium ion from a variety of synthetic cathinones.

Figure 1.3 shows the $\mathrm{MS}^{\mathrm{n}}$ fragmentation of $\alpha$-PVP with the major structural fragments embedded. Isolation and fragmentation of the precursor ion $[\mathrm{M}+\mathrm{H}]^{+}$at $m / z 232$ results in the 
primary product ions at $\mathrm{m} / \mathrm{z}, 214,189,161$, and 154 (Figure 1.3a). The base peak of this spectrum is observed at $\mathrm{m} / \mathrm{z} 161$, which is formed through the loss of the pyrrolidine ring from the precursor ion. Figure 1.3b shows the product ions produced from the isolation and fragmentation of the primary product ion at $m / 2161$. The main product ions are observed at $m / 2,143,133,119,105$, and 91.
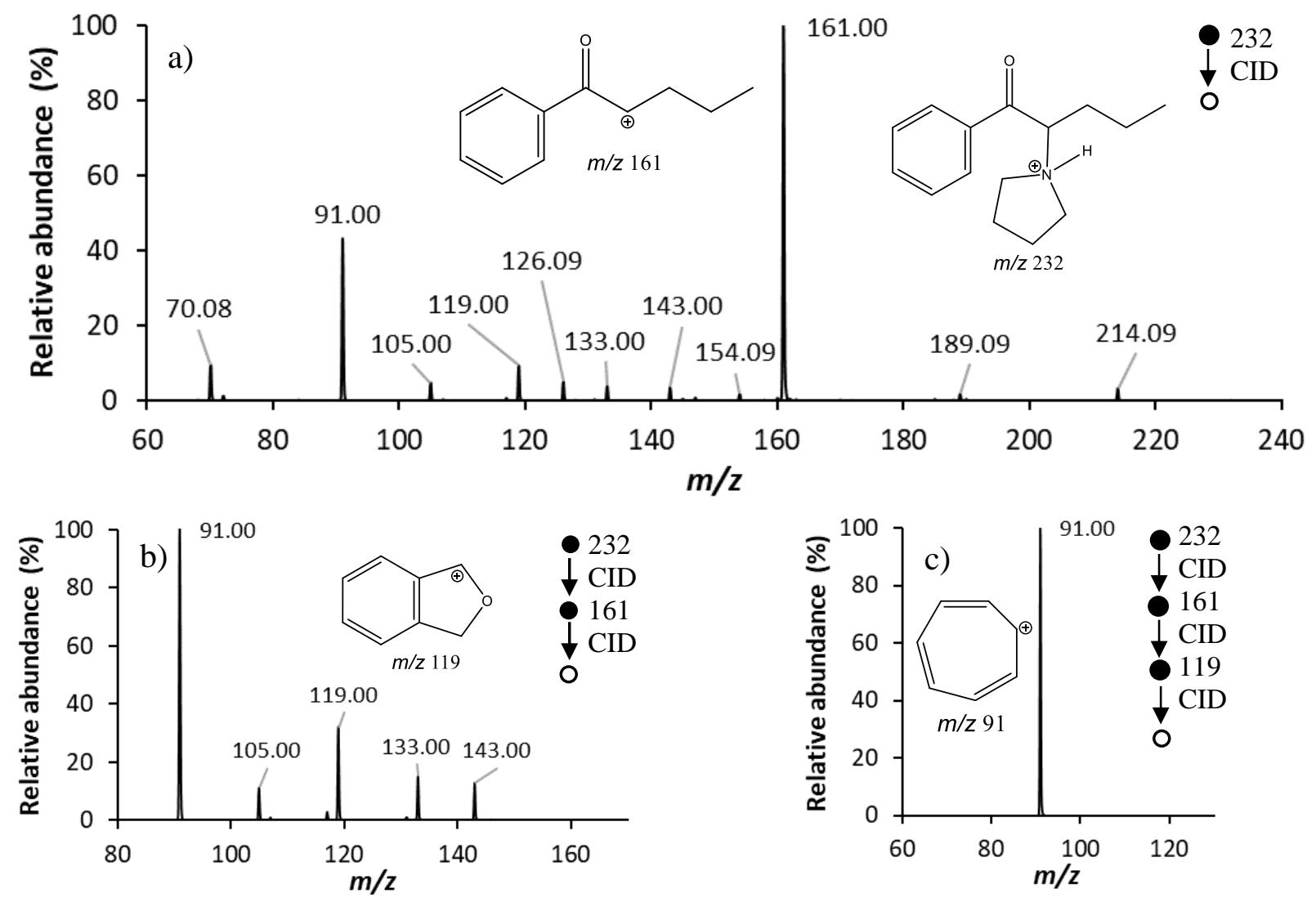

Figure 1.3. Tandem mass spectra of $\alpha-\mathrm{PVP}$ : a) $\mathrm{MS}^{2}$ product ion spectrum of the $[\mathrm{M}+\mathrm{H}]^{+}$ molecular ion (35\% NCE); b) $\mathrm{MS}^{3}$ product ion spectrum of the product ion at $\mathrm{m} / \mathrm{z} 161$ (30\% NCE) showing the formation of product ions at $\mathrm{m} / \mathrm{z}, 143,133,119,105$, and 91 ; c) $\mathrm{MS}^{4}$ product ion spectrum of the secondary product ion at $m / z 119$ (30\% NCE) showing the formation of only the tropylium ion at $m / z$ 91. Evidence for the phthalane structure shown in panel b) is provided by ion spectroscopy and DFT calculations in section 1.3.2.

Based on the $\mathrm{MS}^{\mathrm{n}}$ analysis of $\mathrm{m} / \mathrm{z} 161$, the secondary product ions at $\mathrm{m} / \mathrm{z} 143,133$, and 119 are formed through the loss of $\mathrm{H}_{2} \mathrm{O}$, ethylene or $\mathrm{CO}$, and propylene, respectively. Figure 1.3c shows 
the isolation and fragmentation of the secondary product ion at $m / z 119$, which results in the exclusive formation of the tropylium ion at $\mathrm{m} / \mathrm{z}$, 1 , which can only occur through the loss of CO from the intermediate at $m / z, 119$.

Figure 1.4 shows the $\mathrm{MS}^{\mathrm{n}}$ fragmentation of $\alpha$-PVP that has a ${ }^{13} \mathrm{C}$ label on the carbonyl carbon. The structures of major fragments are also embedded in Figure 1.4. Evidence for the phthalane structure shown in Figure 1.4b is provided by ion spectroscopy and DFT calculations in section 1.3.2. Isolation and fragmentation of the isotope-labeled precursor ion $[\mathrm{M}+\mathrm{H}]^{+}$at $\mathrm{m} / \mathrm{z} 233$ (Figure 1.4a) results in a variety of ions, including $m / z, 162,133,120,106$, and 91 . Following the same logic as Figure 1.3, the structure at $\mathrm{m} / \mathrm{z} 162$ corresponds to the loss of the pyrrolidine ring from the precursor. The product ions formed through the isolation and fragmentation of $m / z \quad 162$ (Figure 1.4b) include $m / z, 144,134,133,120,106,105$, and 91 . Based on $\mathrm{MS}^{\mathrm{n}}$ analyses, the ${ }^{13} \mathrm{C}$-labeled carbonyl carbon is not incorporated into the tropylium ion and must be lost as neutral ${ }^{13} \mathrm{CO}$ (Figure 1.4c). 

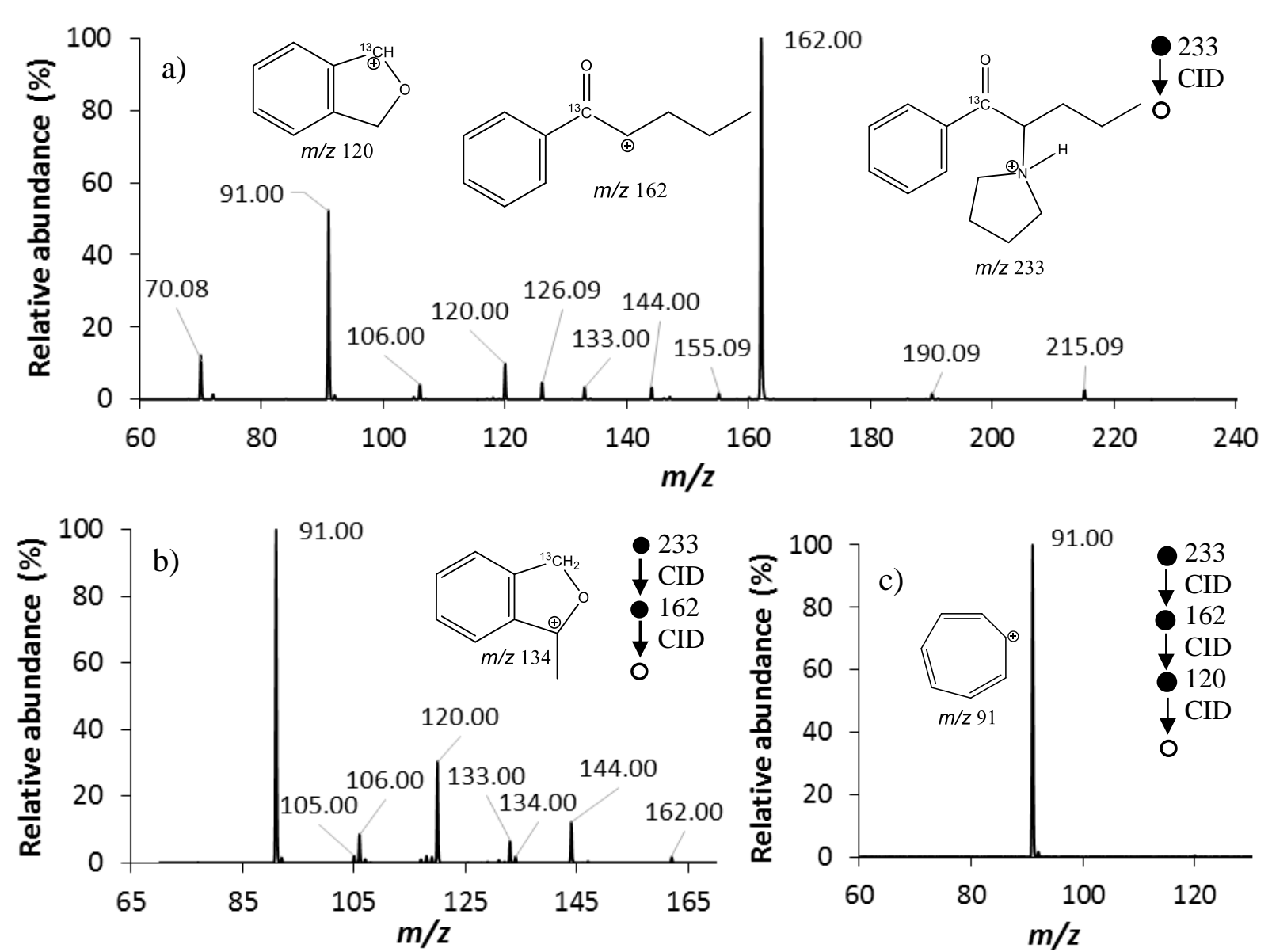

Figure 1.4. Tandem mass spectra of ${ }^{13} \mathrm{C}$-carbonyl labeled $\alpha$-PVP: a) $\mathrm{MS}^{2}$ product ion spectrum of the $[\mathrm{M}+\mathrm{H}]^{+}$molecular ion (35\% NCE); b) $\mathrm{MS}^{3}$ spectrum of the intermediate ion at $\mathrm{m} / z .162$ (30\% NCE); c) $\mathrm{MS}^{4}$ spectrum of the intermediate ion at $\mathrm{m} / z 120$ (30\% NCE) showing the formation of only the secondary product ion at $\mathrm{m} / \mathrm{z}$ 91. Evidence for the phthalane structures in panels a) and b) are provided by ion spectroscopy and DFT calculations in section 1.3.2.

The observation of the intermediate at $m / z 133$ in the $\mathrm{MS}^{2}$ and $\mathrm{MS}^{3}$ spectra of Figure 1.4a and Figure 1.4b, respectively, is particularly interesting. Although the loss of $\mathrm{CO}$ directly from the alkyl chain had been proposed by Pozo et al. [28], Fabregat-Safont et al. [29] and Qian et al. [30], this pathway had not been verified until the current use of isotopic labeling.

The $\mathrm{MS}^{\mathrm{n}}$ fragmentation of the precursor ion $[\mathrm{M}+\mathrm{H}]^{+}$at $m / z 234$ for ${ }^{18} \mathrm{O}-\alpha-\mathrm{PVP}$ results in the formation of primary product ions at $m / z, 214,191,163$, and 156 (Figure 1.5a). The primary product ions, except for $\mathrm{m} / \mathrm{z} 214$, are all shifted by two Daltons (Da) relative to the same product 
ions for $\alpha$-PVP, which appear at $\mathrm{m} / \mathrm{z} 189,161$, and 154 . This observed $2 \mathrm{Da}$ shift indicates these fragments must include the ${ }^{18} \mathrm{O}$-labeled oxygen. In contrast, the primary product ion at $\mathrm{m} / \mathrm{z} 214$ must not contain the ${ }^{18} \mathrm{O}$ label. The product at $\mathrm{m} / \mathrm{z} 214$ can therefore only be explained by the loss of $\mathrm{H}_{2}{ }^{18} \mathrm{O}$ from the precursor.
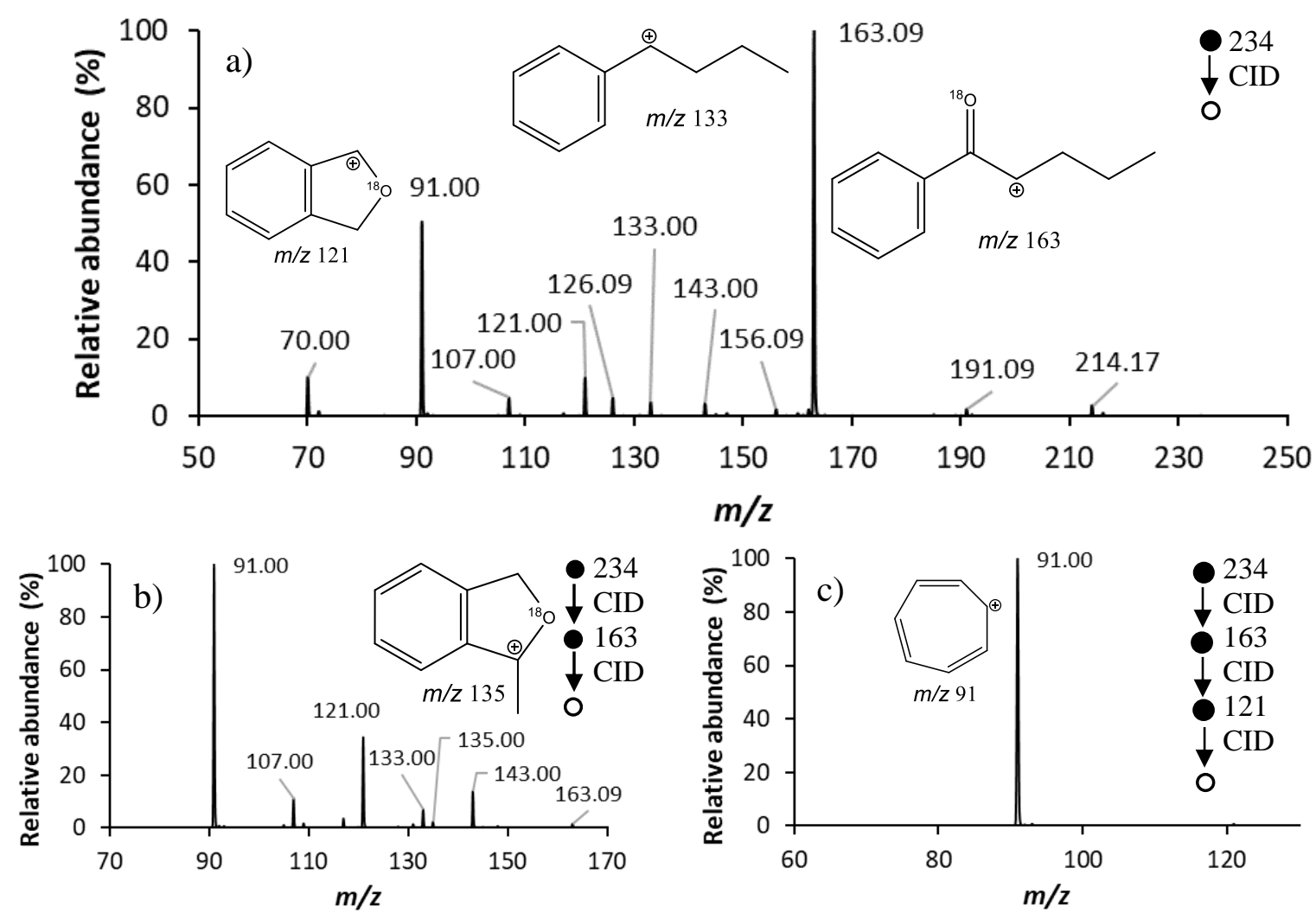

Figure 1.5. Tandem mass spectra of ${ }^{18} \mathrm{O}-\alpha-\mathrm{PVP}$ : a) $\mathrm{MS}^{2}$ product ion spectrum of the $[\mathrm{M}+\mathrm{H}]^{+}$ molecular ion (35\% NCE); b) $\mathrm{MS}^{3}$ spectrum of the product ion at $\mathrm{m} / z 163$ (30\% NCE) showing the formation of product ions at $\mathrm{m} / \mathrm{z}, 143,135,133,121,107$ and 91 ; c) $\mathrm{MS}^{4}$ spectrum of the secondary product ion at $\mathrm{m} / z 121$ (30\% NCE) showing only the formation of tropylium ion at $\mathrm{m} / \mathrm{z}$ 91. Evidence for the phthalane structures in panels a) and b) are provided by ion spectroscopy and DFT calculations in section 1.3.2.

Figure 1.5b shows that the product ion spectrum from the isolation and fragmentation of the primary product ion at $\mathrm{m} / \mathrm{z} 163$ results in product ions at $\mathrm{m} / \mathrm{z} 143,135,133,121,107$, and 91 . The ions at $m / z, 135,121$, and 107 must include the ${ }^{18} \mathrm{O}$ oxygen, whereas the ions at $\mathrm{m} / \mathrm{z} 143,133$, and 91 must not contain the ${ }^{18} \mathrm{O}$ oxygen. A particularly interesting observation is the distribution of the 
secondary product ions at $\mathrm{m} / \mathrm{z} 135$ and $\mathrm{m} / \mathrm{z} 133$, which highlights competing pathways through the loss of ethylene $\left(\mathrm{C}_{2} \mathrm{H}_{4}\right)$ and $\mathrm{C}^{18} \mathrm{O}$ for the loss of 28 or $30 \mathrm{Da}$, respectively, from the primary product ion at $m / z 161$ for $\alpha$-PVP (Figure 1.3b). Isolation and fragmentation of the ${ }^{18} \mathrm{O}$-containing secondary product ion at $m / z 121$ results in only the tropylium ion at $m / z 91$, again consistent with the loss of $\mathrm{CO}$ from the precursor with the elemental composition $\mathrm{C}_{8} \mathrm{H}_{7}{ }^{18} \mathrm{O}^{+}$at $\mathrm{m} / \mathrm{z} 121$ (Figure $1.5 c)$.

Figure 1.6 contains the $\mathrm{MS}^{\mathrm{n}}$ fragmentation of $\alpha-\mathrm{PVP}$ that has a ${ }^{13} \mathrm{C}$ label on the $\alpha$-carbon. Fragmentation of the isotope-labeled precursor ion $[\mathrm{M}+\mathrm{H}]^{+}$at $\mathrm{m} / z 233$ (Figure 1.6a) results in product ions at $m / z 162,144,134,127,120,105$, and 92 . These product ions are entirely consistent with the product ions observed in Figure 1.4a with the ${ }^{13} \mathrm{C}$ label on the carbonyl carbon instead of the $\alpha$-carbon. Figure 1.6b shows the product ions formed through the isolation and fragmentation of the intermediate at $m / z 162$, which includes $m / z$ 144, 134, 120, and 92. Fragmentation of the intermediate ion at $\mathrm{m} / \mathrm{z} 120$ is almost devoid of signal at $\mathrm{m} / \mathrm{z}, 91$ for the all ${ }^{12} \mathrm{C}$-isomer (Figure 1.6c), which indicates that the $\alpha$-carbon is retained and the carbonyl carbon is not. 

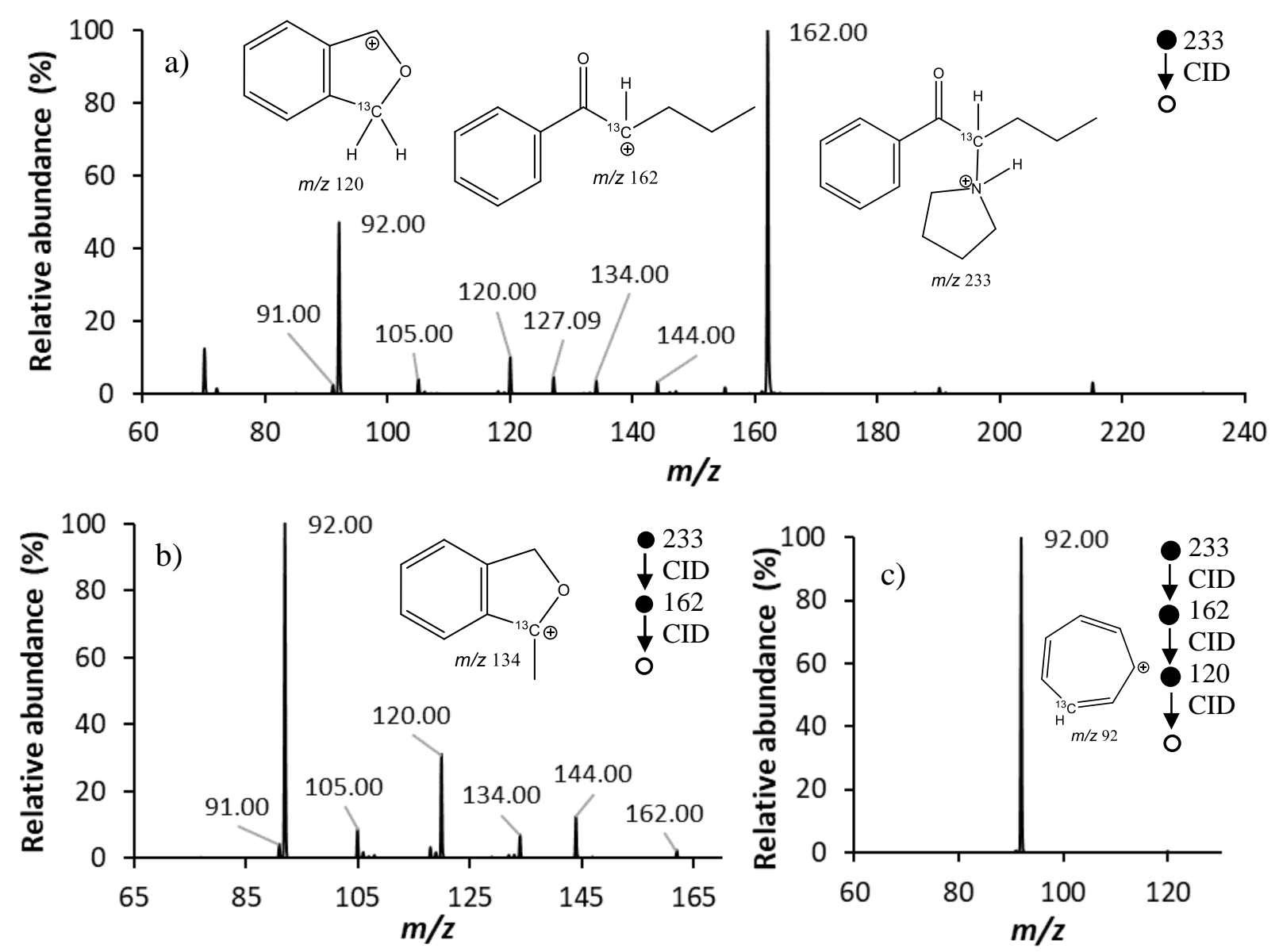

Figure 1.6. Tandem mass spectra of ${ }^{13} \mathrm{C}-\alpha$-carbon $\alpha$-PVP: a) $\mathrm{MS}^{2}$ product ion spectrum of the $[\mathrm{M}+\mathrm{H}]^{+}$molecular ion (35\% NCE); b) $\mathrm{MS}^{3}$ spectrum of the intermediate ion at $\mathrm{m} / \mathrm{z} 162(30 \%$ $\mathrm{NCE}$ ); c) $\mathrm{MS}^{4}$ spectrum of the intermediate ion at $m / z 120$ (30\% NCE) showing the formation of only the product ion at $\mathrm{m} / \mathrm{z}$ 92. Evidence for the phthalane structures in panels a) and b) are provided by ion spectroscopy and DFT calculations in section 1.3.2.

Based on the isotope labeling and $\mathrm{MS}^{\mathrm{n}}$ results, Figure 1.7 shows the proposed fragmentation mechanisms for the generation of the product ions at $m / z 134,133$, and 120 from collisional activation of the protonated molecular ion of ${ }^{13} \mathrm{C}$-carbonyl- $\alpha$-PVP at $m / z 233$. After the loss of the pyrrolidine moiety from the precursor, the ion at $m / z 162$ follows two primary pathways. The first is the loss of ${ }^{13} \mathrm{CO}$ directly from the alkyl chain, which results in the formation of the intermediate at $m / z 133$ (green pathway). The other dominant pathway for the intermediate at $m / z, 162$ is through the formation of an epoxide, which stabilizes the charge on a tertiary carbocation. The epoxide can 
fragment through a variety of charge-remote mechanisms including a 4-center elimination of ethylene to give the product at $\mathrm{m} / \mathrm{z} 134$ (red pathway) and through a different 4-center elimination of propylene to give the product at $m / z 120$ (blue pathway). For the precursor labeled with ${ }^{13} \mathrm{C}$ on the carbonyl carbon, the loss of ${ }^{13} \mathrm{CO}(29 \mathrm{Da})$ and the loss of $\mathrm{C}_{2} \mathrm{H}_{4}(28 \mathrm{Da})$ are readily distinguished. In contrast, the unlabeled precursor provides losses of ${ }^{12} \mathrm{CO}(28 \mathrm{Da})$ and $\mathrm{C}_{2} \mathrm{H}_{4}(28$ Da) have the same nominal mass and are indistinguishable on unit-mass-resolution instruments.

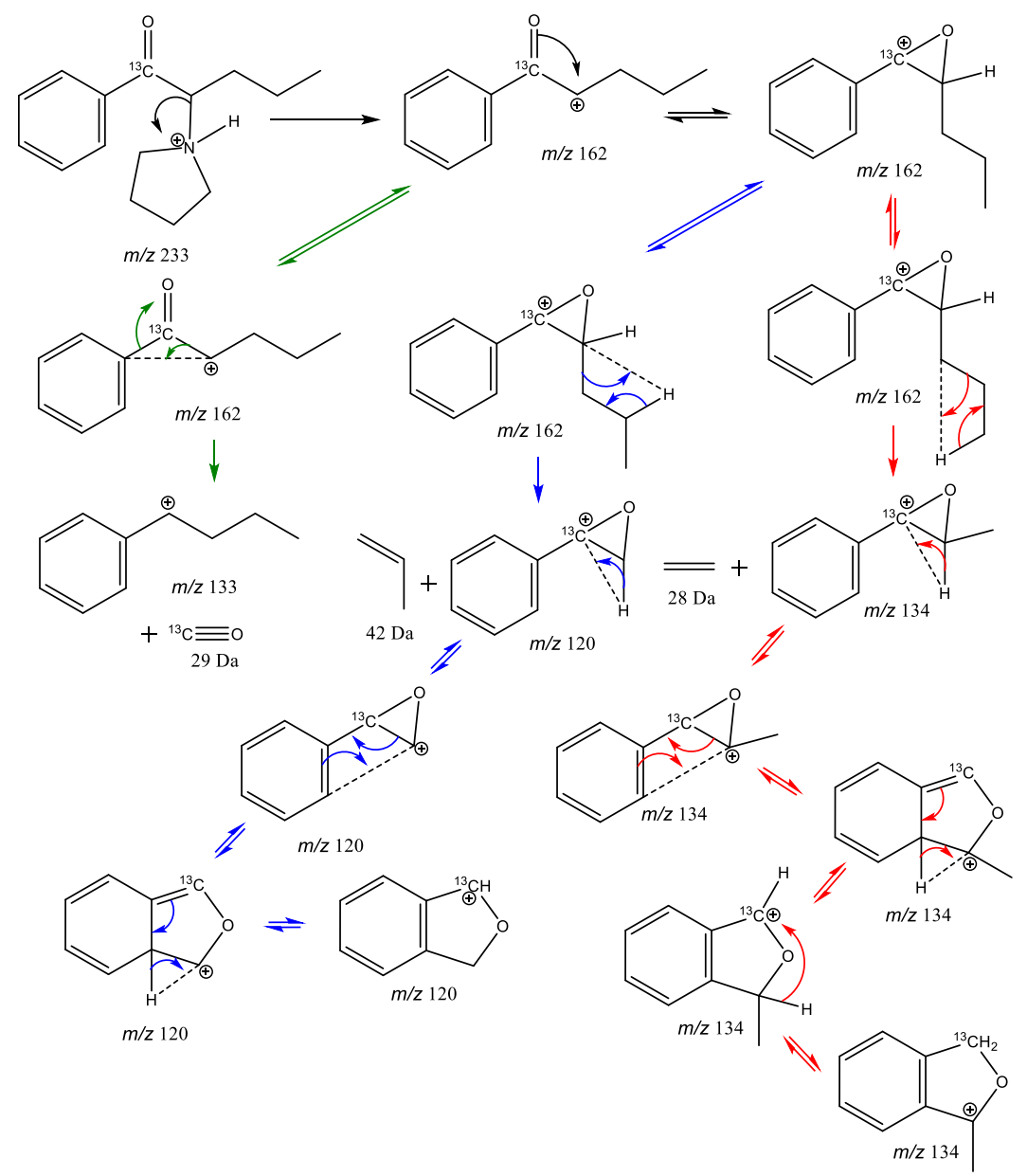

Figure 1.7. Proposed mechanisms for the formation of $\mathrm{m} / \mathrm{z} 134,133$, and 120 product ions from ${ }^{13} \mathrm{C}$-carbonyl carbon- $\alpha$-PVP. The phthalane structures at $\mathrm{m} / \mathrm{z} 120$ and $\mathrm{m} / \mathrm{z} 134$ were confirmed by ion spectroscopy and DFT calculations (see Figure 1.22 and Figure 1.23).

According to DFT calculations, there is no energy barrier to form the epoxide at $\mathrm{m} / \mathrm{z} 162$ in the top right of Figure 1.7 from the secondary carbocation in the top center of Figure 1.7. The epoxide 
carbon distal to the ring can then undergo nucleophilic attack by $\pi$-electrons from the aromatic ring - after or during the loss of an ethylene or propylene neutral loss of 28 or $42 \mathrm{Da}$, respectively - to provide the phthalane core for the product ions at $m / z 134$ and $m / z ~ 120$, respectively. DFT calculations show that the phthalane structure (bottom right structure in Figure 1.7) is thermodynamically the most stable isomer compared to seven alternative isomeric structures. However, energy barriers associated with these isomer interconversions have not been studied. These mechanisms help explain both the presence of the secondary product ions at $\mathrm{m} / \mathrm{z}$ 134 and $\mathrm{m} / \mathrm{z}$ 133, and they are consistent with the ion spectroscopy results for the most probable structures (see section 1.3.2). The competing pathways between the loss of the non $-{ }^{13} \mathrm{C}$-labled ethylene neutral (28 Da) and the ${ }^{13} \mathrm{C}$-labled CO neutral (29 Da) explains the presence of both $\mathrm{m} / \mathrm{z}$ 134 and $m / z 133$ in the product ion spectrum of ${ }^{13} \mathrm{C}$-carbonyl carbon- $\alpha$-PVP.

Figure 1.8 shows two possible mechanisms for the formation of the product ions at $\mathrm{m} / \mathrm{z} 91$ and $\mathrm{m} / \mathrm{z}, 92$ from the intermediate at $\mathrm{m} / \mathrm{z}$ 120. The conversion energetics of the last few steps-from the benzylium ion to the tropylium ion-have been described in detail by Vala et al. [49]. These pathways are based on the ${ }^{13} \mathrm{C}$ isotopic labeling of both the carbonyl carbon and $\alpha$-carbon, and they help explain the observations that the $\alpha$-carbon is incorporated into the tropylium ion through the loss of neutral CO containing the carbonyl carbon. According to the acquired spectra, the alkyl hydrogens must have a sufficiently high barrier for rearrangement to prevent the phthalane structures at the top of Figure 1.8 from interconverting. The two fragments shown at the top of Figure 1.8 are therefore distinct and not in equilibrium. 


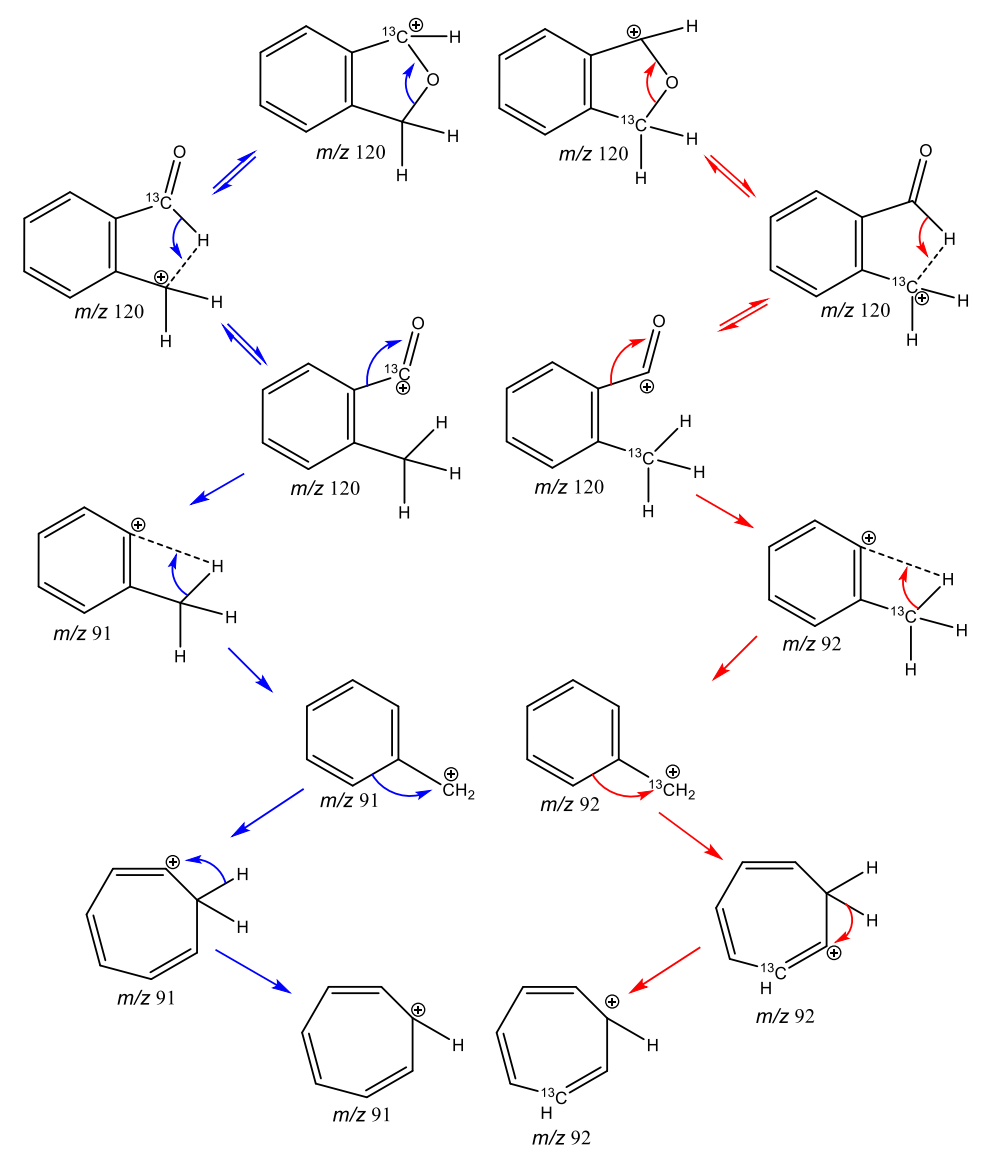

Figure 1.8. Proposed mechanisms for the formation of product ions at $m / z 91$ and $m / z 92$ from the intermediate at $m / z 120$ for $\mathrm{MS}^{4}$ based on ${ }^{13} \mathrm{C}$ isotopic labeling of the carbonyl carbon and the $\alpha$-carbon for $\alpha$-PVP.

Figure 1.9a shows the $\mathrm{MS}^{3}$ product ion spectrum from the intermediate product ion at $\mathrm{m} / \mathrm{z} 134$ for ${ }^{13} \mathrm{C}$-carbonyl carbon- $\alpha$-PVP. This spectrum highlights the formation of product ions at $m / z$ 106,105 , and 92 . The corresponding $\mathrm{MS}^{3}$ product ion spectrum for the intermediate product ion at $m / z 133$ for ${ }^{13} \mathrm{C}$-carbonyl carbon- $\alpha-\mathrm{PVP}$ is shown in Figure 1.9b. Figure 1.9b demonstrates the formation of product ions at only $\mathrm{m} / \mathrm{z} 105$ and $\mathrm{m} / \mathrm{z}$ 91. Figure 1.10 shows the proposed mechanisms for the formation of the $\mathrm{m} / \mathrm{z} 106,105,92$, and 91 product ions from the intermediate product ions at $m / z 134$ and $m / z 133$ for ${ }^{13} \mathrm{C}$-carbonyl carbon- $\alpha$-PVP. Figure 1.11 shows the $\mathrm{MS}^{3}$ fragmentation of the intermediate product ion at $m / z 134$ from ${ }^{13} \mathrm{C}-\alpha$-carbon- $\alpha$-PVP and Figure 1.12 demonstrates the proposed mechanisms to explain the product ions at $m / z$ 106, 105, 92, and 91 . 

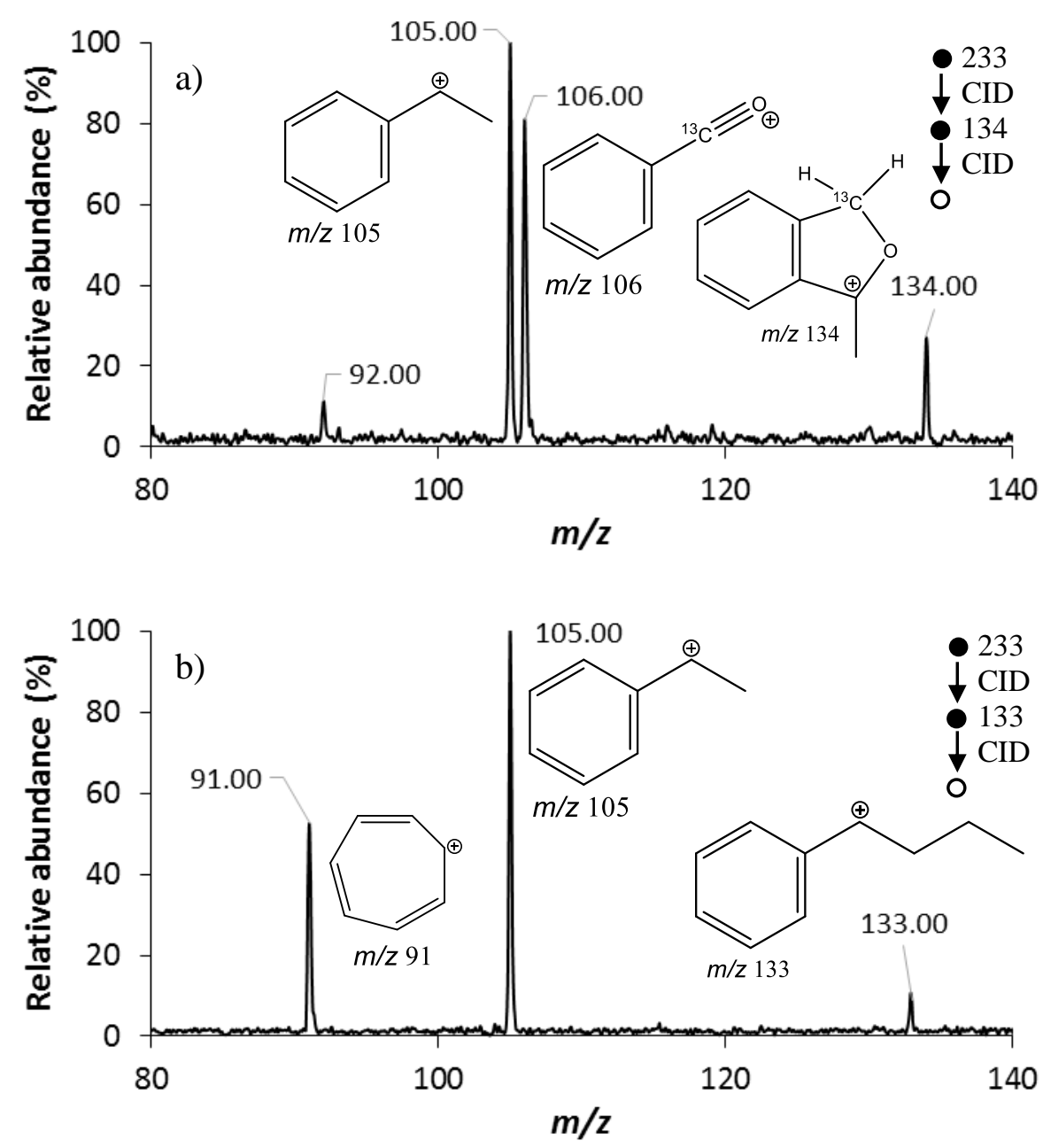

Figure 1.9. $\mathrm{MS}^{3}$ product ion mass spectra of: a) the product ion at $\mathrm{m} / \mathrm{z} 134$ (30\% NCE) showing the formation of product ions at $\mathrm{m} / \mathrm{z} 106,105$, and 92 from ${ }^{13} \mathrm{C}$-carbonyl carbon- $\alpha$-PVP and $\mathrm{b}$ ) the product ion at $m / z 133(30 \% \mathrm{NCE})$ showing the presence of only the product ions at $\mathrm{m} / z 105$ and $m / z, 91$ from ${ }^{13} \mathrm{C}$-carbonyl carbon- $\alpha$-PVP. 


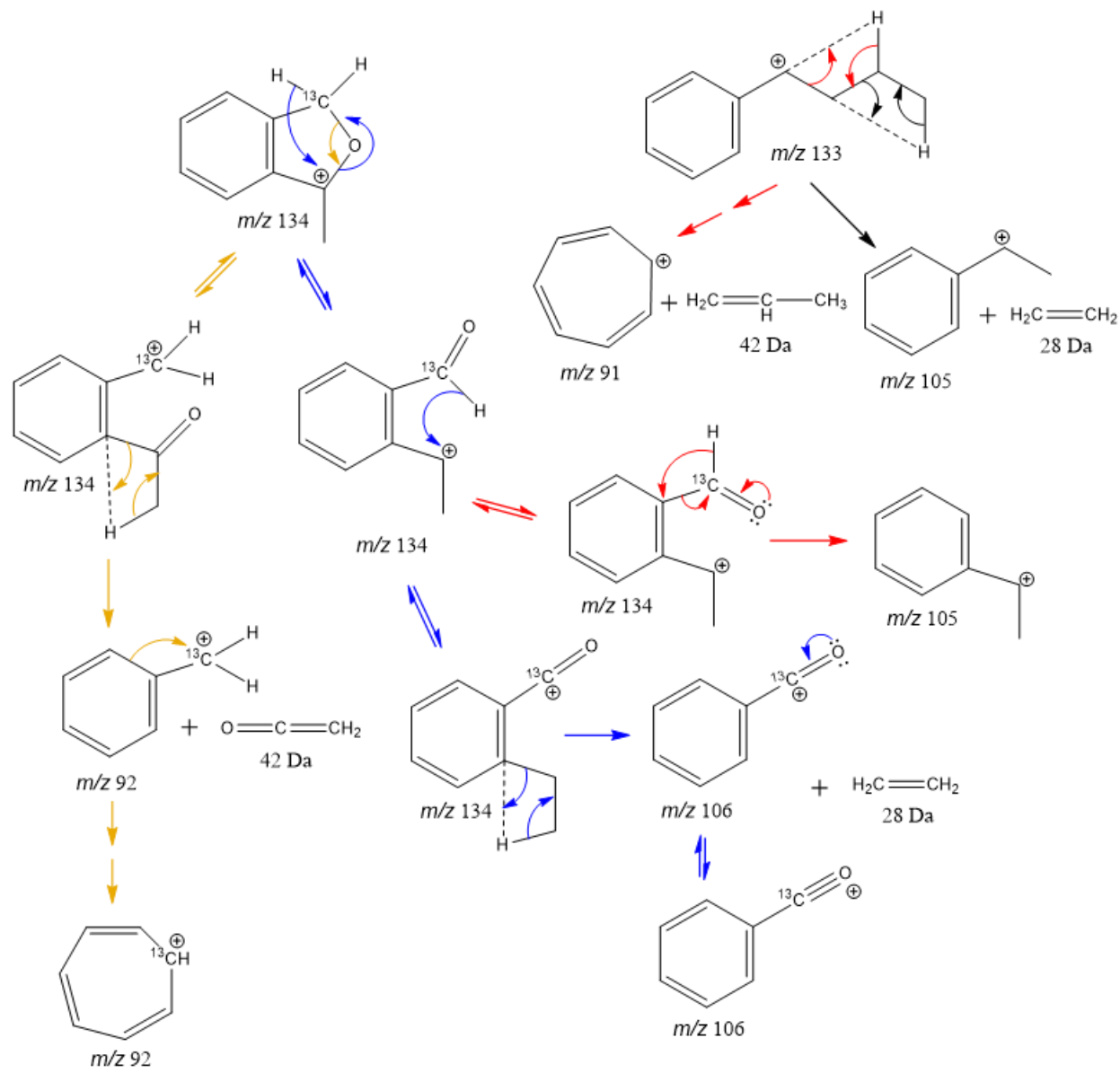

Figure 1.10. Proposed mechanisms for the formation of the product ions from the intermediate ions at $m / z, 134$ and $m / z, 133$ as observed from the $\mathrm{MS}^{3}$ analysis for ${ }^{13} \mathrm{C}$-carbonyl carbon- $\alpha-\mathrm{PVP}$.

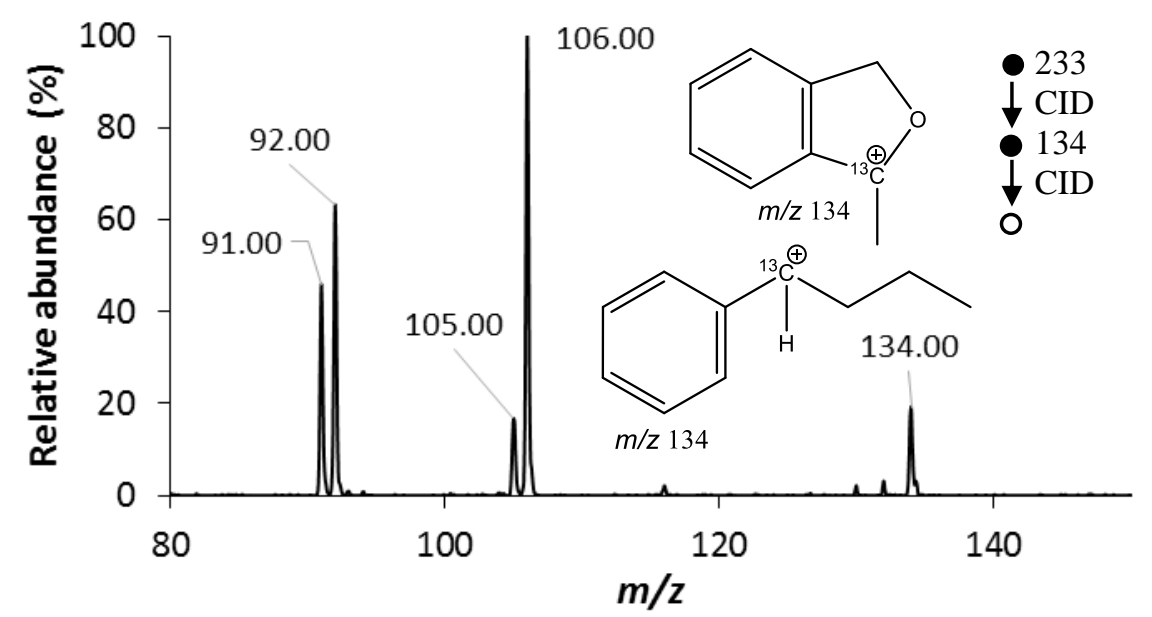

Figure 1.11. $\mathrm{MS}^{3}$ product ion mass spectra of the product ion at $\mathrm{m} / z$ 134 (30\% NCE) showing the formation of product ions at $m / z 106,105,92$ and 91 from ${ }^{13} \mathrm{C}-\alpha$-carbon- $\alpha$-PVP. 


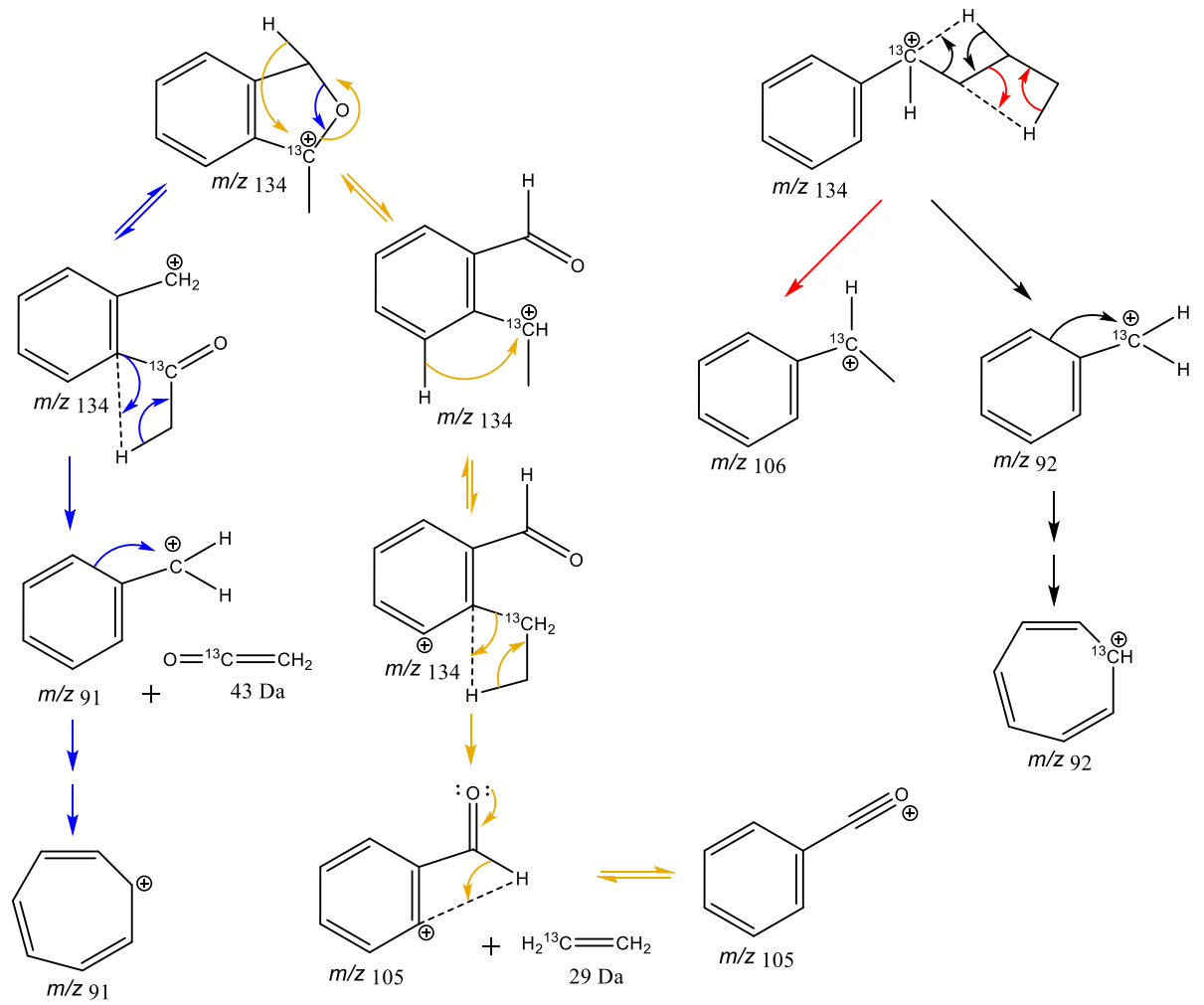

Figure 1.12. Proposed mechanisms for the formation of the product ions at $\mathrm{m} / \mathrm{z}, 106,105,92$, and 91 from the $\mathrm{MS}^{3}$ analysis of the intermediate ion at $m / z 134$ from ${ }^{13} \mathrm{C}-\alpha$-carbon- $\alpha$-PVP.

The analysis of $\alpha-\mathrm{PVP}-\mathrm{d}_{7}$, which is perdeuterated along the alkyl chain, provides additional support for the proposed mechanisms shown in Figure 1.7 and Figure 1.8. When $\alpha$-PVP is perdeuterated along the alkyl chain, the $[\mathrm{M}+\mathrm{H}]^{+}$precursor ion is observed at $\mathrm{m} / z 2239$, and fragmentation of the $\mathrm{d}_{7}$ precursor results in abundant fragments at $\mathrm{m} / \mathrm{z}, 168,140,120,93$ and 92 , among others (Figure 1.13a). Isolation and fragmentation of the intermediate at $\mathrm{m} / \mathrm{z} 168$ (Figure 1.13b) shows an interesting distribution of fragments around $\mathrm{m} / \mathrm{z}, 92$ and $\mathrm{m} / \mathrm{z}, 120$. The formation of the ion at $m / z 92$ must occur through the incorporation of a single deuterium into the tropylium ion, whereas the formation of ions at $\mathrm{m} / z 93$ and $\mathrm{m} / z 94$ must involve the incorporation of two and three deuteriums, respectively, into the tropylium ion. This same pattern of deuterium inclusion is observed for the intermediates at $\mathrm{m} / \mathrm{z}, 120,121$, and 122. The mechanims described in Figure 1.7 
and Figure 1.8 are consistent with the experimental observations regarding scrambling along the alkyl chain $(\mathrm{m} / \mathrm{z} 93$ and $\mathrm{m} / \mathrm{z} 121)$ and the aromatic ring $(\mathrm{m} / \mathrm{z} 94$ and $\mathrm{m} / \mathrm{z} 122)$.

Based on the results for the fragmentation of $\alpha$-PVP labeled with ${ }^{18} \mathrm{O}$, one would expect $\alpha$ PVP- $\mathrm{d}_{7}$ to lose either ${ }^{12} \mathrm{CO}(28 \mathrm{Da})$ or $\mathrm{C}_{2} \mathrm{D}_{4}(32 \mathrm{Da})$ from the intermediate at $\mathrm{m} / z 168$ to provide product ions at $\mathrm{m} / \mathrm{z} 140$ and $\mathrm{m} / \mathrm{z} 136$, respectively. Although both are observed, only the intermediate product ion a $\mathrm{m} / \mathrm{z}, 140$ is readily observed in the full-scale plot. These observations are consistent with both the $\mathrm{C}_{9} \mathrm{H}_{9} \mathrm{O}^{+}$and $\mathrm{C}_{10} \mathrm{H}_{13}{ }^{+}$intermediate product ions in unlabeled $\alpha$-PVP, which are discussed later in the high-resolution mass spectrometry section.

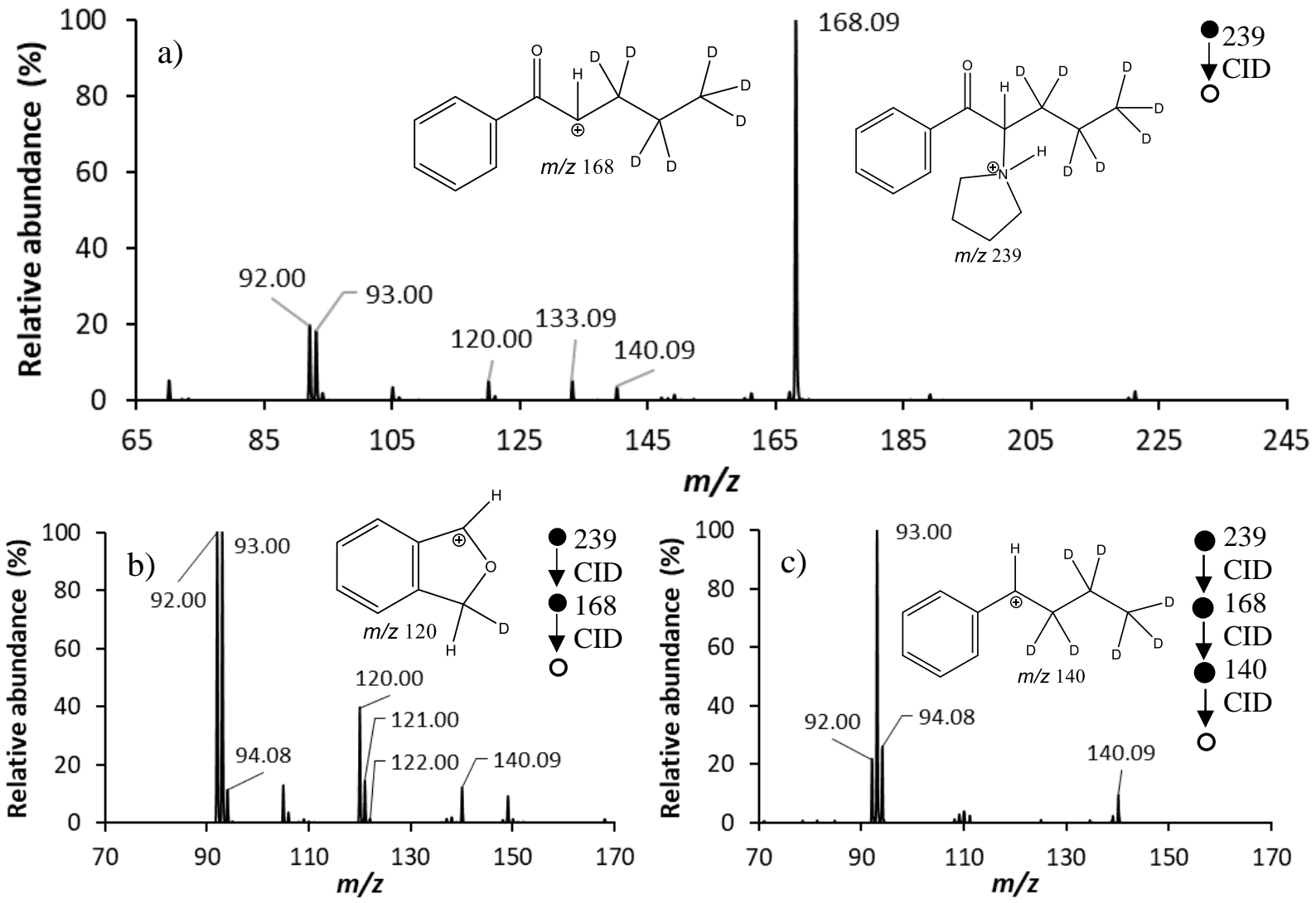

Figure 1.13. Tandem mass spectra of $\alpha-P V P-d_{7}$ : a) $\mathrm{MS}^{2}$ product ion spectrum of the $[\mathrm{M}+\mathrm{H}]^{+}$ molecular ion $(35 \% \mathrm{NCE})$; b) $\mathrm{MS}^{3}$ product ion spectrum of the product ion at $\mathrm{m} / \mathrm{z} 168(30 \%$ NCE) showing the formation of secondary product ions at $m / z, 140,122,121,120,94,93$, and 92; c) $\mathrm{MS}^{4}$ product ion spectrum of the intermediate at $\mathrm{m} / z, 140$ (30\% NCE) showing the formation of secondary product ions at $\mathrm{m} / \mathrm{z}$ 92, 93, and 94 . 
ESI-MS/MS analysis of other cathinone structures showed that the alkyl chain length has a direct impact on the formation of the tropylium ion and associated intermediate product ions. As the alkyl chain length increases, additional intermediates are possible, and they also contribute to the formation of the tropylium ion. For example, PV8 has two additional methylene groups relative to $\alpha$-PVP. The $[\mathrm{M}+\mathrm{H}]^{+}$precursor of PV8 is observed at $m / z 260$, and the major product ions appear at $m / z 189,147,133,119$ and 91 . Consistent with the other pyrrolidine-containing cathinones, the structure of the intermediate product ion at $\mathrm{m} / \mathrm{z} 189$ corresponds to the preferred loss of the pyrrolidine ring from the precursor. The structure of the intermediate product ion at $\mathrm{m} / \mathrm{z} 119$ is presumably the same as in Figure 1.3, which likely has the same phthalane structure as shown in Figure 1.7 and Figure 1.8. The mass of the intermediate product ion at $m / 2,147$ suggests that it corresponds to the fragment at $\mathrm{m} / \mathrm{z} 133$ (described in Figure 1.4, Figure 1.5, Figure 1.7) with an additional methylene group $\left(\mathrm{CH}_{2}\right)$. Secondary fragmentation of the intermediate at $\mathrm{m} / \mathrm{z} \quad 147$ provides secondary product ions at $m / z 119$ and $\mathrm{m} / \mathrm{z} 91$.

Product ion spectra change more dramatically when the alkyl chain length is decreased relative to $\alpha$-PVP. For example, Figure 1.14 shows ESI-MS/MS spectra of cathinones with both shorter and branched alkyl chains and their ability to prevent the formation of the tropylium ion. Our results show that when the alkyl chain attached to the aromatic ring is at least four carbons long (including the carbonyl cabon), the tropylium ion is observed, which is consistent with previous results $[\underline{18}, \underline{22}, \underline{37}, \underline{50]}$. However, as a general rule, when the alkyl chain is shorter than four carbon atoms, the formation of the tropylium ion is severely inhibited. These observations are explained by the need for a sufficient number of carbons on the alkyl appendage to enable both sterically favorable rearrangements and a good leaving group for the phthalane ring to form, as is the case for $\alpha-P V P$ in Figure 1.7. Finally, Figure 1.14 shows that even when the alkyl chain contains four 
carbon atoms, the incorporation of a methyl group on the $\alpha$-carbon also quenches the mechanism for tropylium ion formation. Quenching of the tropylium ion formation is an expected outcome of the mechanism shown in Figure 1.7 because the additional methyl group on the $\alpha$-carbon both stabilizes the charge on the $\alpha$-carbon and provides steric hinderance to the nucleophilic attack by the $\pi$-electrons from the aromatic ring. 

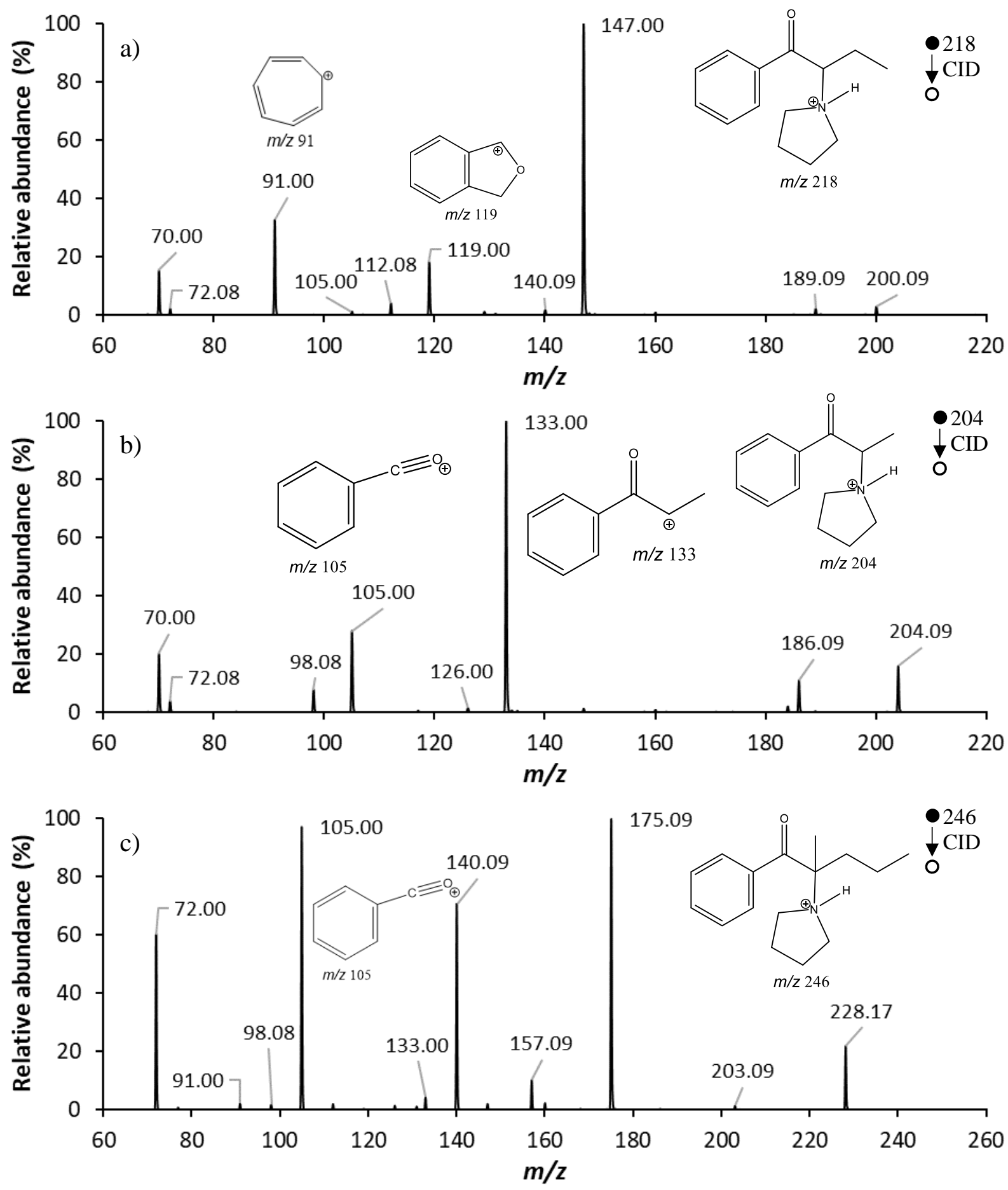

Figure 1.14. Tandem mass spectra of: a) $\alpha$-PBP (35\% NCE), b) $\alpha$-PPP (30\% NCE), and c) $\alpha$-PVPmethyl at the $\alpha$-carbon (35\% NCE) demonstrating the presence of the tropylium ion at $\mathrm{m} / z$, 91 for $\alpha$-PBP, absence for $\alpha$-PPP, and essential absence (<2\%) for $\alpha$-PVP-methyl ( $\alpha$-carbon). 
Figure 1.15 shows the ESI-MS/MS spectrum of protonated 3,4-MDPV-d $\mathrm{d}_{8}$. The spectrum indicates that the deuterium atoms on the pyrrolidine ring are not incorporated into the benzene ring during the skeletal rearrangement and instead remain on the pyrrolidine moiety. The absence of deuterium scrambling is confirmed with the product ion at $m / z 134$, which is an $8 \mathrm{Da}$ mass increase relative to the 1-butylidenepyrrolidin-1-ium product ion at $m / z 126$ observed for nondeuterated 3,4-MDPV (Figure 1.15a). When the $[\mathrm{M}+\mathrm{H}]^{+}$precursor of 3,4-MDPV-d 8 at $m / z, 284$ is isolated and fragmented, the base peak is the secondary product ion at $m / z 205$ (Figure 1.15a). This ion is a 44 Da mass increase relative to the secondary product ion at $m / z, 161$ of $\alpha$-PVP, which corresponds to the additional mass of the methylenedioxy substituent, as expected.

$\mathrm{MS}^{3}$ fragmentation of the base peak at $m / z 205$ for 3,4-MDPV-d produces secondary product ions at both $m / z 177$ and $m / z$ 163, which both represent a 44 Da mass increase relative to the corresponding non-methylenedioxy substitutions observed for $\alpha$-PVP (Figure 1.15b). These fragments also support the conserved nature of the proposed fragmentation mechanisms that are apparently unperturbed by modifications on the aromatic ring. The product ion at $\mathrm{m} / \mathrm{z} 175$ forms through the loss of formaldehyde $\left(\mathrm{CH}_{2} \mathrm{O}\right)$, which comes from the methylenedioxy substituent and explains why this equivalent fragment is not observed for $\alpha$-PVP. Figure 1.15c shows the isolation and fragmentation of the intermediate product ion at $m / z 177$ for 3,4-MDPV- $\mathrm{d}_{8}$, which highlights the loss of both propylene (42 Da) and formaldehyde (30 Da). Again, the formaldehyde loss from 3,4-MDPV comes from the methylenedioxy substituent, which is not present for $\alpha$-PVP. 

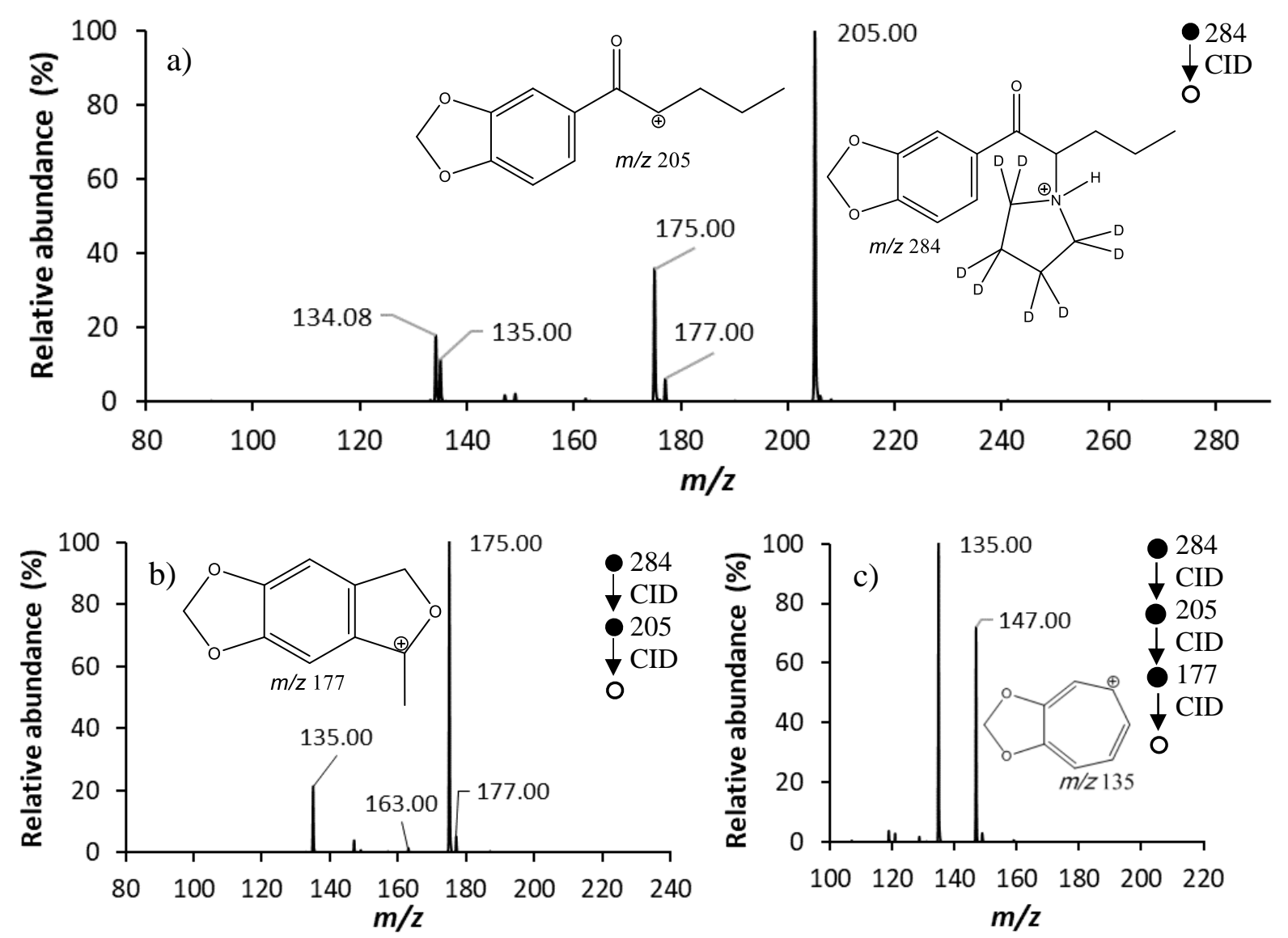

Figure 1.15. Tandem mass spectra of $3,4-\mathrm{MDPV}-\mathrm{d}_{8}$ : a) $\mathrm{MS}^{2}$ product ion spectrum of the $[\mathrm{M}+\mathrm{H}]^{+}$molecular ion $(35 \% \mathrm{NCE})$; b) $\mathrm{MS}^{3}$ product ion spectrum of the product ion at $\mathrm{m} / \mathrm{z} 205$ (35\% NCE) showing the formation of secondary product ions at $\mathrm{m} / \mathrm{z} 177,163$ and 135 ; and c) $\mathrm{MS}^{4}$ product ion spectrum of the product ion at $\mathrm{m} / z 177$ (35\% NCE) showing the formation of the tropylium ion derivative at $\mathrm{m} / \mathrm{z} 135$.

Figure 1.16 compares the tandem mass spectra of the $[\mathrm{M}+\mathrm{H}]^{+}$precursor ion for 3,4-MDPV and the $[\mathrm{M}+\mathrm{H}]^{+}$precursor ion for 2,3-MDPV, both of which are observed at $m / z 276$. In both cases, the major fragments leading to the formation of the substituted tropylium ions are observed at $\mathrm{m} / \mathrm{z}$ 205, 177, 163 and 135. However, the isomers have different fragment ion abundances at $\mathrm{m} / \mathrm{z} 205$ and $m / z$ 135. When the methylenedioxy substituent is in the 3,4-positon, the intermediate product ion at $m / z 205$ is the base peak of the tandem mass spectrum and the product ion at $\mathrm{m} / \mathrm{z} 135$ is present at $\sim 10 \%$ abundance. In contrast, when the methylenedioxy substituent is in the 2,3position, the intermediate product ion at $m / z 205$ is only about $40 \%$ of the base peak and the product 
ion at $m / z 135$ is about $70 \%$ of the base peak. This behavior highlights two trends about the position of the methylenedioxy substituent: 1) the formation of the tropylium ion is favored for the 2,3position, and 2) the loss of formaldehyde from the intermediate product ion $\mathrm{m} / \mathrm{z} 205$ is favored for the 2,3-positon.
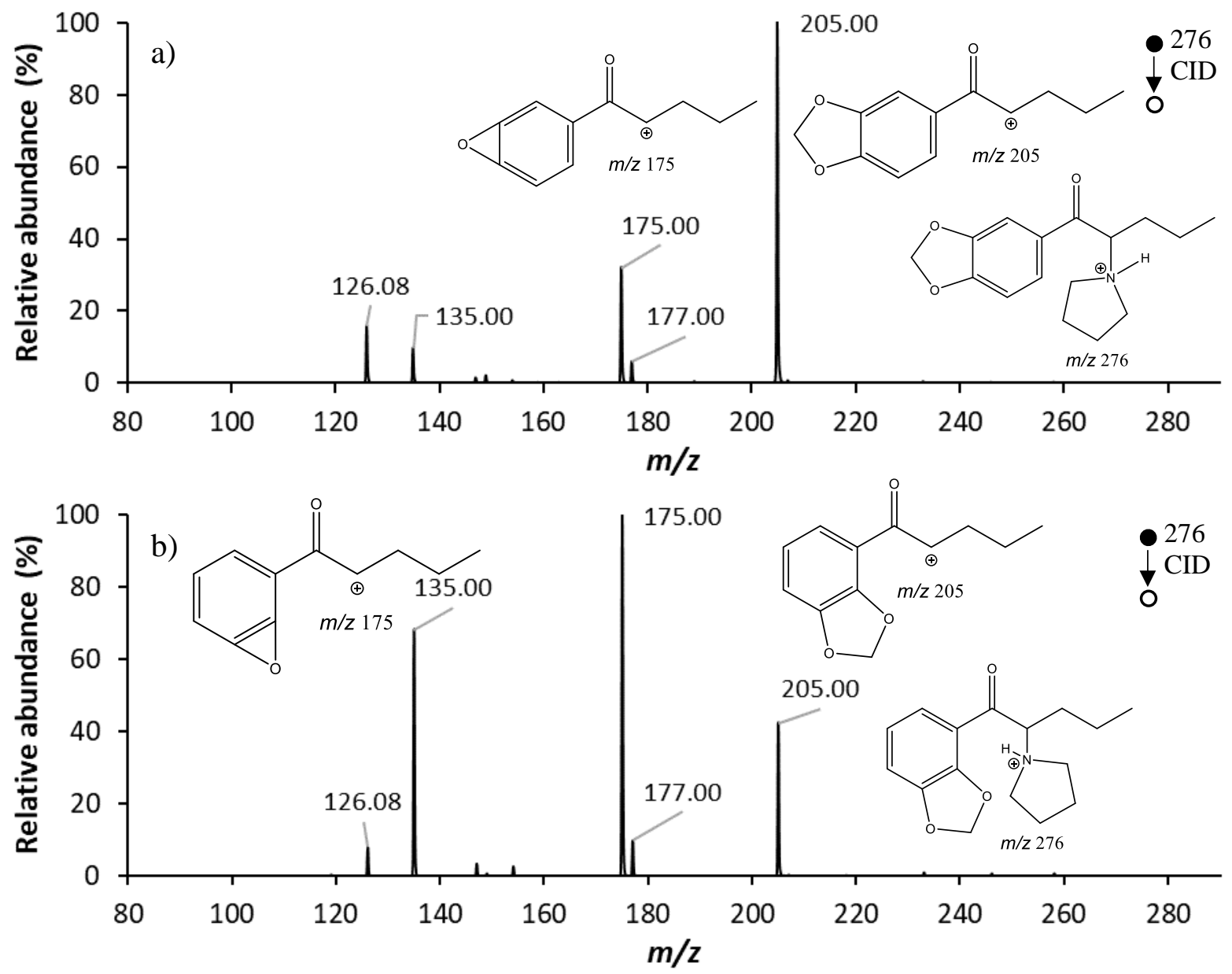

Figure 1.16. Tandem mass spectra of positional isomers highlighting the impact of substituent location as demonstrated by a) 3,4-MDPV (35\% NCE) and b) 2,3-MDPV (35\% NCE) demonstrating the differences in fragmentation based on location of the methylenedioxy substituent.

Figure 1.17 shows the pathways from the $[\mathrm{M}+\mathrm{H}]^{+}$precursor to the final tropylium product ion along each major pathway for $\alpha$-PBP, $\alpha$-PVP, and PV8. The flux is expressed as a percentage of the total ion spectrum. As discussed previously, when the alkyl chain length increases there are 
additional intermediate product ions that feed into the tropylium ion pathway. Likewise, the conversion rate from precursor to intermediate changes as a function of possible pathways, where $\alpha$-PBP contains only two intermediates and PV8 contains four intermediates along the tropylium ion pathways. The length of the alkyl chain has a direct effect on the conversion rates; the intermediate product ion at $m / z, 133$ for $\alpha$-PVP having a $79 \%$ conversion to the tropylium product ion at $m / z$ 91, whereas the intermediate product ion at $\mathrm{m} / z, 133$ from PV8 has only a $1 \%$ conversion rate to the tropylium product ion at $\mathrm{m} / \mathrm{z}$ 91. Figure $\mathbf{1 . 1 7}$ also shows that the conversion rate for the intermediate product ion at $\mathrm{m} / \mathrm{z} 119$ to the tropylium product ion at $\mathrm{m} / \mathrm{z} 91$ is $\sim 100 \%$ for the three unsubstituted cathinones.

a)<smiles>CCC(C(=O)c1ccccc1)N1CCCC1</smiles>

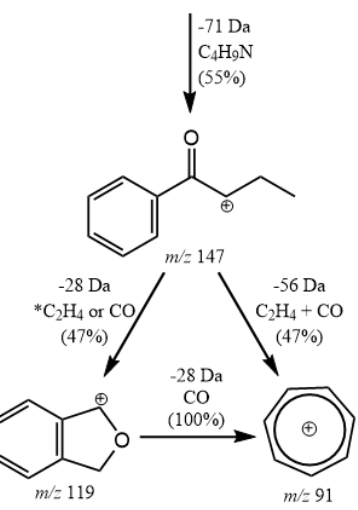

*Proposed structure drawn b)

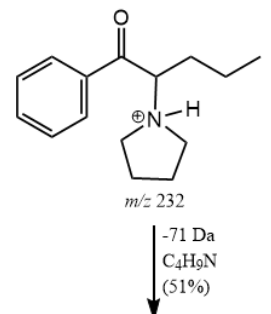

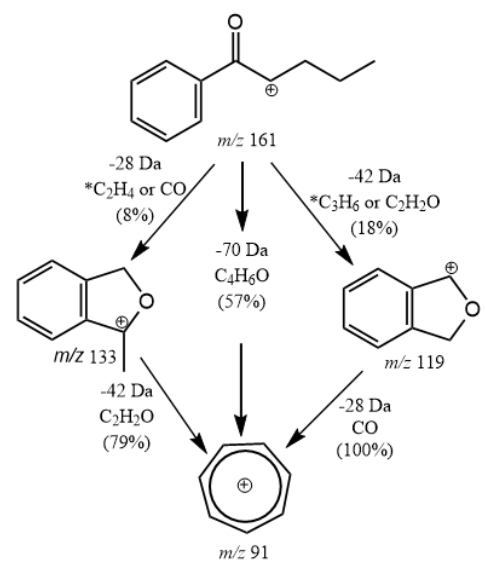

$m / 291$ c)
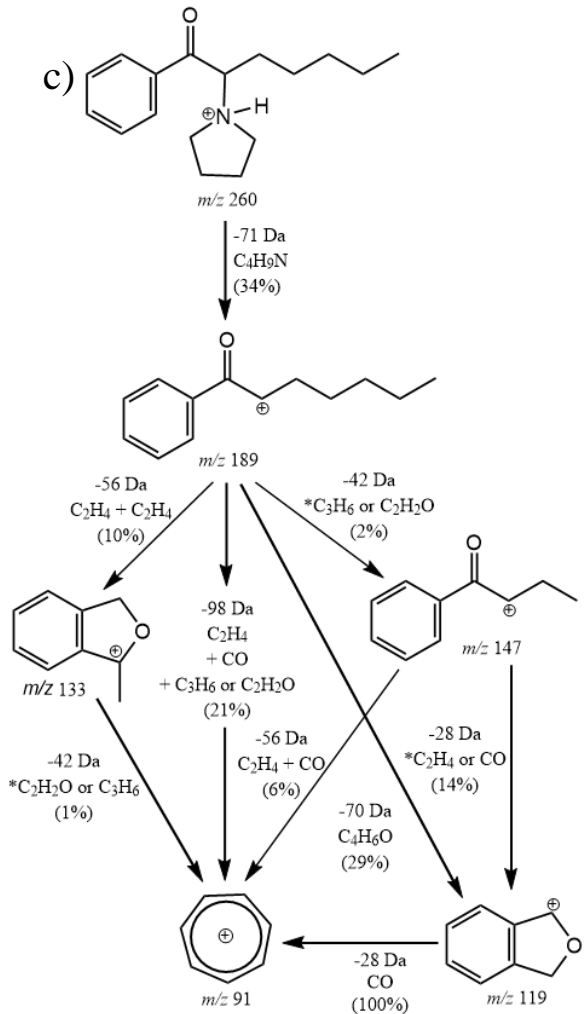

Figure 1.17. Selected fragmentation pathways and corresponding flux for: a) $\alpha-P B P, b) \alpha-P V P$, and c) PV8. The percentages shown at each level of $\mathrm{MS}^{\mathrm{n}}$ provide the ion's abundance relative to the summed ion abundance of the product ion spectrum at that level. For example, $m / z, 147$ is the base peak (100\% peak height) in the $\mathrm{MS}^{2}$ product ion spectrum of $\alpha$-PBP and $55 \%$ of the summed product ion spectrum. 


\subsubsection{High-Resolution Mass Spectrometry (HRMS) Measurements using ESI-Q-TOF}

Whereas the IT mass spectrometer offered the capability to perform multiple stages of mass spectrometry $\left(\mathrm{MS}^{\mathrm{n}}\right)$, HRMS allowed for accurate mass measurements from the tandem mass spectra. Accurate mass measurements are useful because they provide unique elemental compositions, with our typical instrument uncertainty on the order of $10 \mathrm{ppm}$ or about $3 \mathrm{mDa}$. The ability to identify the elemental composition of a fragment becomes particularly important when there are multiple ways to explain a neutral loss, such as $\mathrm{CO}$ and $\mathrm{C}_{2} \mathrm{H}_{4}$, which both have the nominal mass of $28 \mathrm{Da}$. Figure 1.18a shows the high resolution tandem mass spectra of $\alpha$-PVP with the major structural fragments embedded.
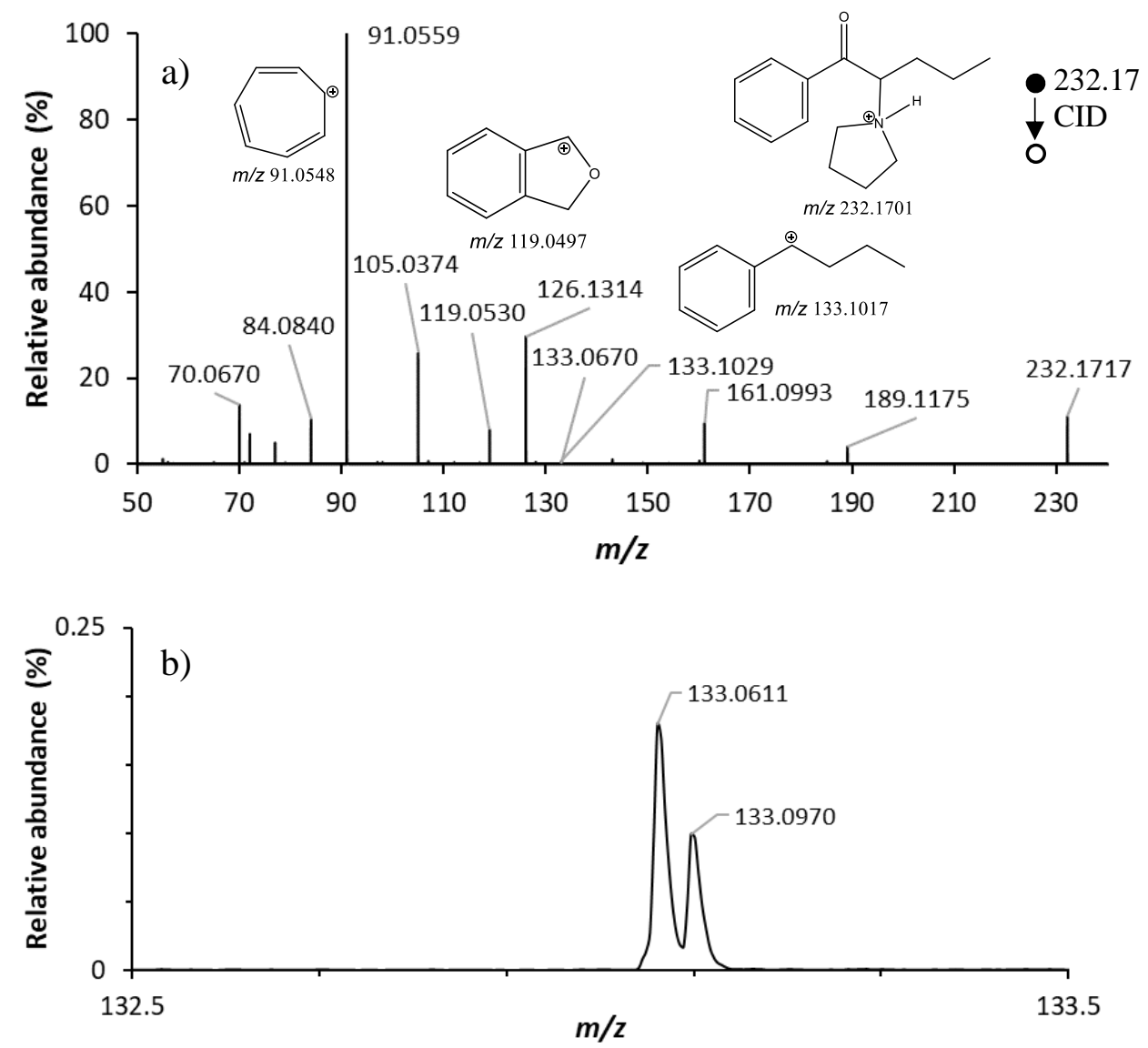

Figure 1.18. Tandem mass spectra of $\alpha$-PVP showing: a) the fragmentation observed on the QTOF mass spectrometer with a $25 \mathrm{eV}$ collision energy and b) a zoomed-in view of product ions at $\mathrm{m} / \mathrm{z} 133.0611$ and $\mathrm{m} / \mathrm{z} 133.0970$ with a $35 \mathrm{eV}$ collision energy. 
The HRMS tandem mass spectrum of $\alpha$-PVP allows for the determination of the elemental formula of four important ions. The first is the tropylium ion with an accurate mass of $\mathrm{m} / \mathrm{z} 91.0559$, which is about $12 \mathrm{ppm}$ from the exact mass of $\mathrm{C}_{7} \mathrm{H}_{7}^{+}$. The second important ion is the proposed phthalane structure with an accurate mass of $\mathrm{m} / \mathrm{z}$ 119.0530, which deviates $28 \mathrm{ppm}$ from the exact mass for $\mathrm{C}_{8} \mathrm{H}_{7} \mathrm{O}^{+}$. Finally, the HRMS measurements of the two product ions with a nominal mass of $\mathrm{m} / \mathrm{z} 133$ in Figure 1.18a had elemental compositions of both $\mathrm{C}_{9} \mathrm{H}_{9} \mathrm{O}^{+}$(measured at $\mathrm{m} / \mathrm{z}$ 133.0670; expected at $\mathrm{m} / \mathrm{z}, 133.0653 ; 13 \mathrm{ppm}$ error) and $\mathrm{C}_{10} \mathrm{H}_{13}{ }^{+}$(measured at $\mathrm{m} / 2$ 133.1029; expected at $m / z$ 133.1017; 9 ppm error). Although CID in the HRMS instrument tends to favor the formation of fragments at smaller $\mathrm{m} / \mathrm{z}$ values than in the lower-energy IT instrument, the lowabundance $\mathrm{C}_{10} \mathrm{H}_{13}{ }^{+}$product ion was slightly less abundant than the $\mathrm{C}_{9} \mathrm{H}_{9} \mathrm{O}^{+}$product ion under 35 eV CID conditions (Figure 1.18b). The combination of the ${ }^{18} \mathrm{O}-\alpha-\mathrm{PVP}$ IT results (Figure 1.5) and the HRMS results provide unequivocal evidence for the competing pathways between the loss of $\mathrm{CO}$ and ethylene for the formation of the product ion with a nominal mass of $m / z 133$.

Figure 1.19 shows the tandem mass spectra of PV8 with the major structural fragments embedded. Although the alkyl chain is two carbons longer for PV8 than $\alpha-\mathrm{PVP}$, the same core tropylium ion fragmentation ions are observed for both $\alpha$-PVP and PV8. Based on the HRMS accurate mass measurements, the error between the accurate mass measurements and exact masses is on the order of those discussed in Figure 1.18. The additional intermediate at $\mathrm{m} / \mathrm{z} 147.0838$ is hardly above the noise level at the conditions used for this experiment; however, the accurate mass measurement is consistent with the elemental formula $\mathrm{C}_{10} \mathrm{H}_{11} \mathrm{O}^{+}$. 


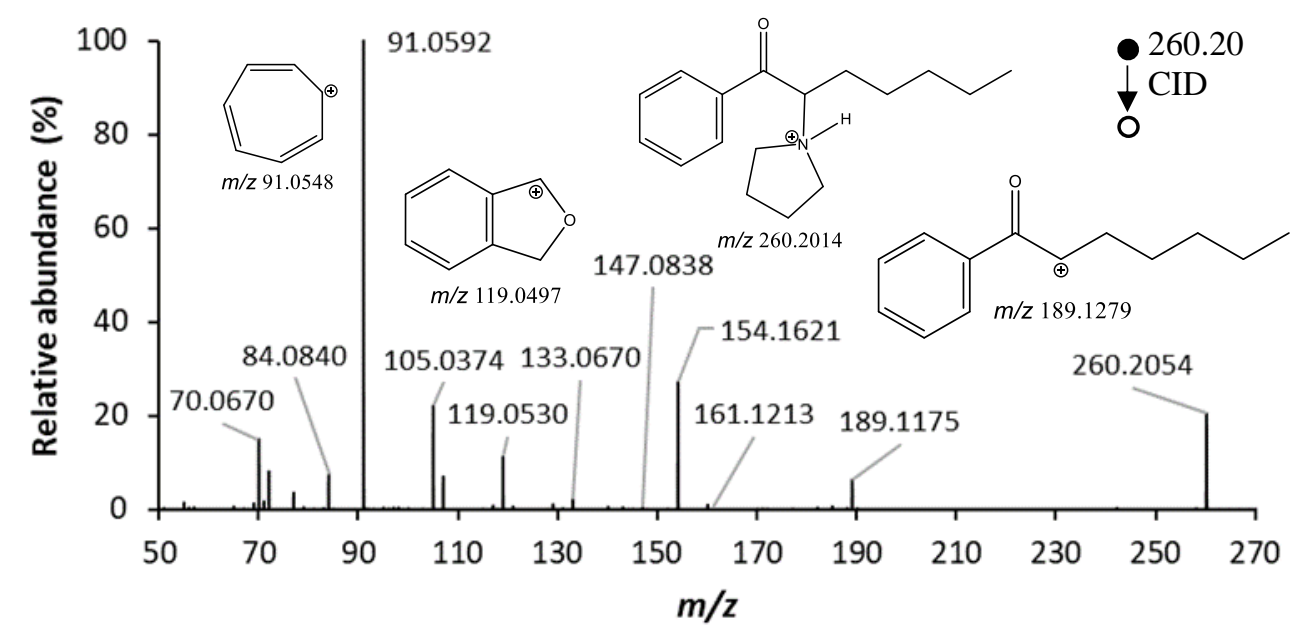

Figure 1.19. Tandem mass spectra of PV8 $(25 \mathrm{eV})$ showing the conserved nature of the loss of the pyrrolidine moiety with the Q-TOF mass spectrometer and the propensity to form the tropylium ion.

Figure 1.20 contains an additional example of the tandem mass spectra generated with the QTOF mass spectrometer for 3,4-MDPV.

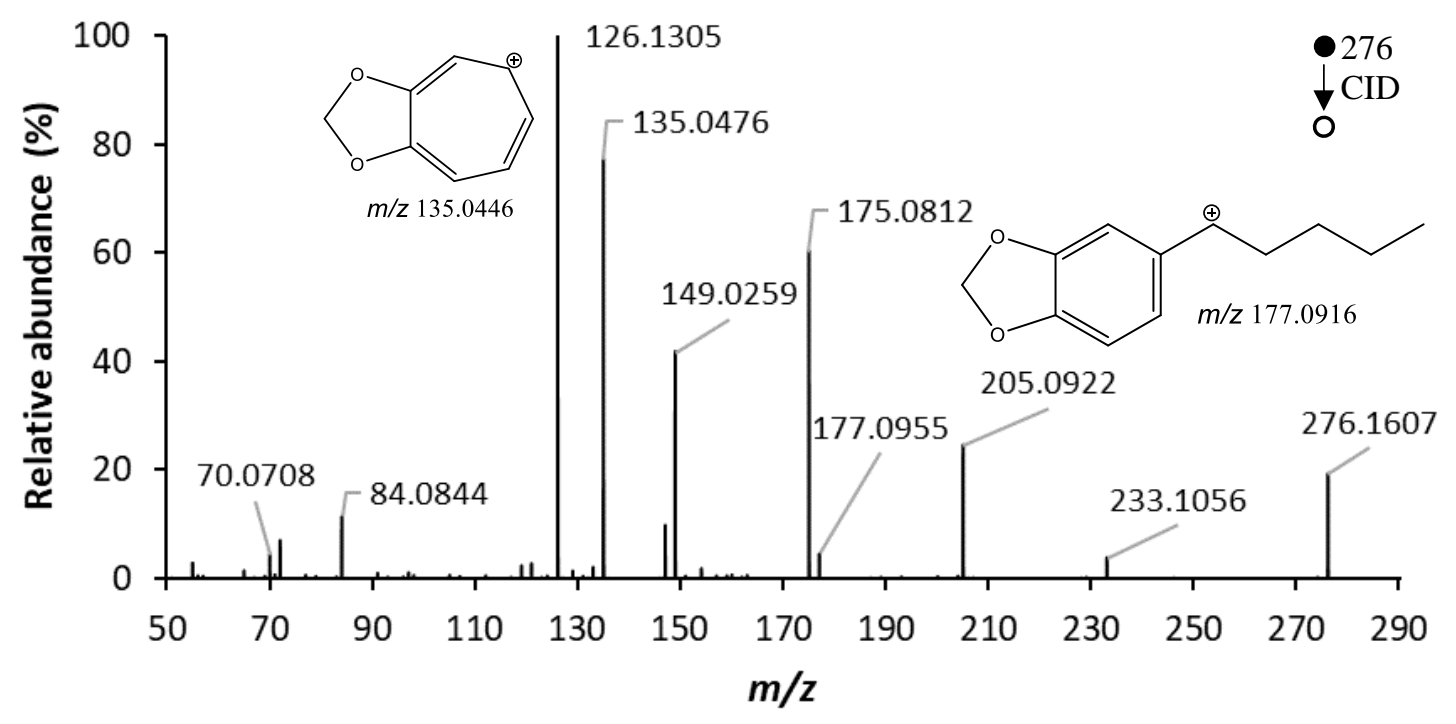

Figure 1.20. Tandem mass spectrum of 3,4-MDPV ( $25 \mathrm{eV}$ collision energy) showing the propensity to form the substituted tropylium ion is conserved in a wide variety of substituted cathinones.

Based on the accurate mass measurements from the Q-TOF mass spectrometer and the $\mathrm{MS}^{\mathrm{n}}$ fragmentation from the IT mass spectrometer, the following generalized trends can be made about 
the fragmentation behavior of $\alpha$-pyrrolidinophenone synthetic cathinones along the tropylium ion or substituted-tropylium ion pathways. The loss of the pyrrolidine moiety always forms the first intermediate ion along this pathway, as shown by the product ion at $m / z 189$ for PV8. From this intermediate, there are competing pathways for the loss of $\mathrm{CO}$ and ethylene with the loss of $\mathrm{CO}$ favored for all compounds, observed at $m / z 161$ (more visible on the IT instrument than the QTOF instrument). Additionally, any compound with a chain length of at least four carbons can form intermediates through the loss of $28 \mathrm{Da}$ (i.e. product ion at $\mathrm{m} / \mathrm{z} 133$ for PV8) and $42 \mathrm{Da}$ with the loss of $42 \mathrm{Da}$ always corresponding with the loss of propylene (i.e. product ion at $\mathrm{m} / \mathrm{z} 147$ for PV8). The intermediate formed through the loss of propylene feeds directly into the tropylium ion pathway through the loss of $28 \mathrm{Da}$ (ethylene) in all cases studied, as shown by the product ion at $\mathrm{m} / \mathrm{z} 119$ for all cathinones. Finally, the intermediate ion formed through the loss of propylene forms the tropylium ion at $m / z 91$ through the loss of $\mathrm{CO}$ for all cathinones.

The differences in the mass spectra generated with IT and Q-TOF mass spectrometers are beyond the focus of the current project. However, in brief, the IT mass spectrometer favors the formation of lower energy and higher mass intermediate ions relative to the Q-TOF mass spectrometer, which included some higher-energy pathways like the loss of alkyl radicals to form odd-electron product ions. These spectral differences stem from the well-known differences in the collision energy, number of collisions, and activation time scales between the IT and Q-TOF mass spectrometers. The IT fragmentation process involves trapping CID (very slow activation, i.e. 10$100 \mathrm{~ms}$ ) through hundreds of collisions with the bath gas, whereas the Q-TOF fragmentation occurs through low-energy (slow activation, i.e. 0.5-1 ms) beam-type collisions (i.e. 10-100) as the analyte passes through the collision cell $[\underline{51}, \underline{52}]$. 
In general, the mass spectra collected with both the IT and Q-TOF mass spectrometers were sufficiently similar that they are cross-comparable and the fragmentation pathways were conserved across all synthetic cathinones analyzed with one notable exception. The fragmentation behavior of ${ }^{13} \mathrm{C}$-carbonyl carbon-4'-methyl- $\alpha$-PHP (MPHP) was somewhat unique. The $\mathrm{MS}^{2}$ spectrum of ${ }^{13} \mathrm{C}-\mathrm{MPHP}$ is different than the fragmentation described above, even though the chemical structure meets all the requirements for the rearrangements involved in the formation of the tropylium ion. Figure 1.21 shows the tandem mass spectrum of ${ }^{13} \mathrm{C}-\mathrm{MPHP}$, which is dominated by the product ion at $m / z$ 105.0727. This fragment must have the elemental composition $\mathrm{C}_{8} \mathrm{H}_{9}{ }^{+}$and must form after the loss of ${ }^{13} \mathrm{CO}$ directly from the alkyl chain. The reason for this deviation in fragmentation behavior is probably that the methyl substitution on the aromatic ring favors the formation of the product ion at $m / z 105.0727$ rather than the tropylium product ion at $m / z 91.0565$. The impact of this information is that it allows for a quick and easy manner for the detection of a methylsubstitution to the aromatic ring for substituted synthetic cathinones, which will be dominated by the product ion at $m / z 105$ instead of the product ion at $\mathrm{m} / \mathrm{z}, 91$. The ratio of $\mathrm{m} / \mathrm{z} .105$ to $\mathrm{m} / \mathrm{z} 91$ is readily detectable, even with mass spectrometers with unit mass resolution. 


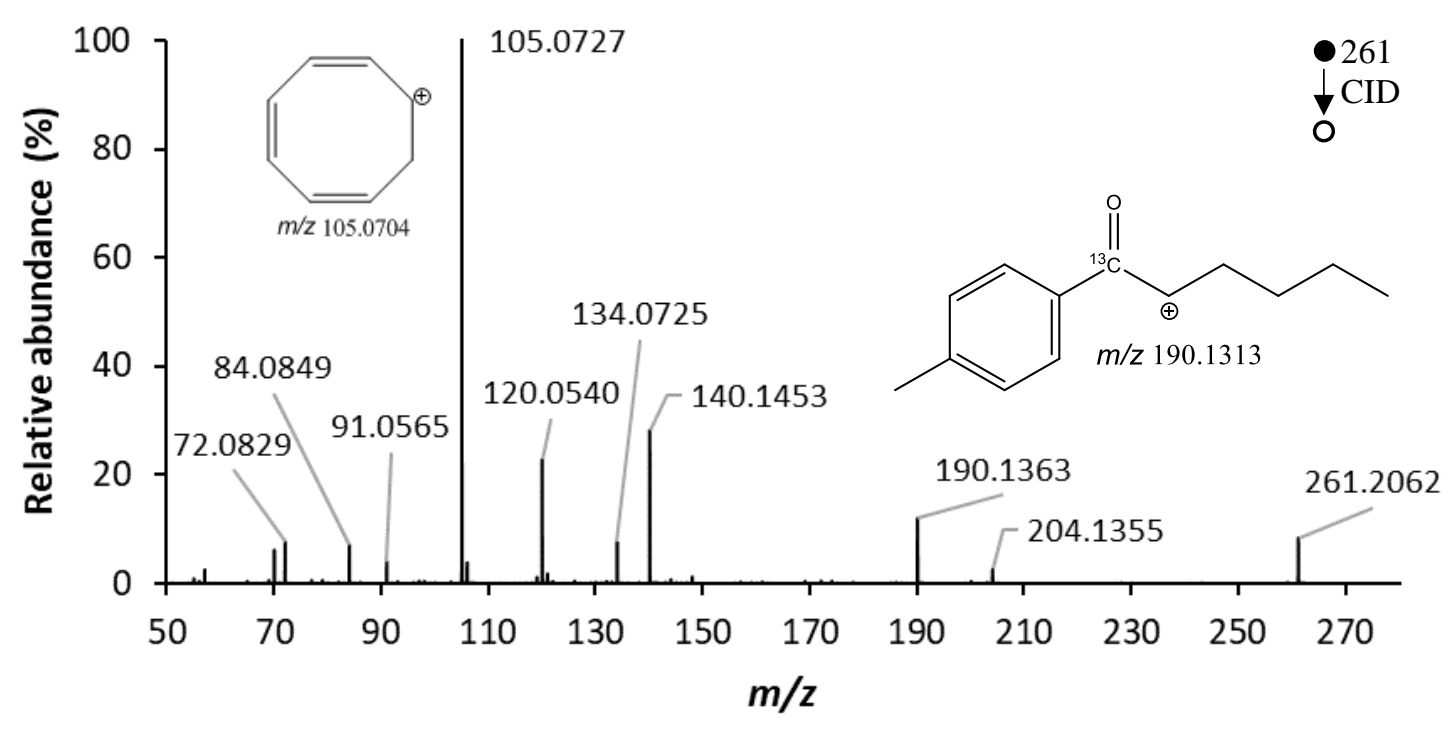

Figure 1.21. Tandem mass spectra of ${ }^{13} \mathrm{C}-\mathrm{MPHP}(25 \mathrm{eV}$ collision energy) showing the dominant abundance of $\mathrm{m} / z 105.0727$ for the $\mathrm{C}_{8} \mathrm{H}_{9}{ }^{+}$ion relative to $\mathrm{m} / z 91.0565$ for the $\mathrm{C}_{7} \mathrm{H}_{7}{ }^{+}$tropylium ion. The structure of the ion at $\mathrm{m} / z 105$ could equally take the form of a methyl-substituted tropylium ion.

\subsubsection{Infrared Ion Spectroscopy}

The combination of the $\mathrm{MS}^{\mathrm{n}}$ and accurate mass measurement results determined the relationship between each intermediate product ion of interest and the tropylium ion, but also the elemental formula of those intermediates. However, neither of these techniques allow for the determination of the exact arrangement of the atoms present in each intermediate. To help answer the question of constitutional arrangement, infrared ion spectroscopy was employed to characterize the intermediates at $\mathrm{m} / \mathrm{z} 119$ and $\mathrm{m} / \mathrm{z} 133$ for $\alpha$-PVP.

Figure 1.22 shows a comparison between the experimentally observed gas-phase IR spectra and the theoretically calculated DFT spectra for two proposed structures for the intermediate product ion at $m / z$ 119. Based on being the lowest-energy isomer from eight isomeric structures evaluated (Table 1.1), and the similarity in wavenumber between the experimental and theoretical calculation, the structure labeled 119a was identified as the most likely structure for the 
intermediate product ion at $\mathrm{m} / \mathrm{z} 119$. We note here that the IR-induced dissociation was energetically demanding, requiring 10 FEL pulses at high IR laser pulse energy, probably as a consequence of the compact nature of the interrogated structures. We suspect these high thresholds in combination with non-linearities in the multiple-photon excitation process lead to a skewed frequency dependence of the fragment yield, reducing the observed intensities in the IRMPD spectra towards lower photon energies.

The structure $119 \mathrm{~d}$ is an example of a poor fit both in terms of relative energy of formation $(208 \mathrm{~kJ} / \mathrm{mol}$ relative to structure 119a) and alignment of the experimental and theoretical spectra. The principal feature of the calculated spectra of $119 \mathrm{~d}$ at $\sim 1850 \mathrm{~cm}^{-1}$ can be assigned to the stretching of the carbonyl bond. The highly coupled stretch of the $C \alpha-C \beta$ bonds is responsible for both the peaks at $\sim 1600 \mathrm{~cm}^{-1}$ and $1400 \mathrm{~cm}^{-1}$. Based on the experimental spectra, we know that the intermediate product ion at $\mathrm{m} / \mathrm{z} 119$ cannot contain a carbonyl because of the absence of the characteristic feature at $1850 \mathrm{~cm}^{-1}$, which is well beyond the experimentally observed peak at 1600 $\mathrm{cm}^{-1}$. The additional peaks in the measured spectrum between $\sim 1100-1500 \mathrm{~cm}^{-1}$ derive from C-H wagging of $\mathrm{CH}_{2}$ hydrogens. The absence of the feature at the carboxylic absorption wavelength and the good match of the peak at $\sim 1600 \mathrm{~cm}^{-1}$, assigned to a highly-coupled C-C stretching of aromatic carbon bonds, along with the most favorable thermodynamics, make isomer 119a the most probable structure of the $m / z 119$ fragment. 

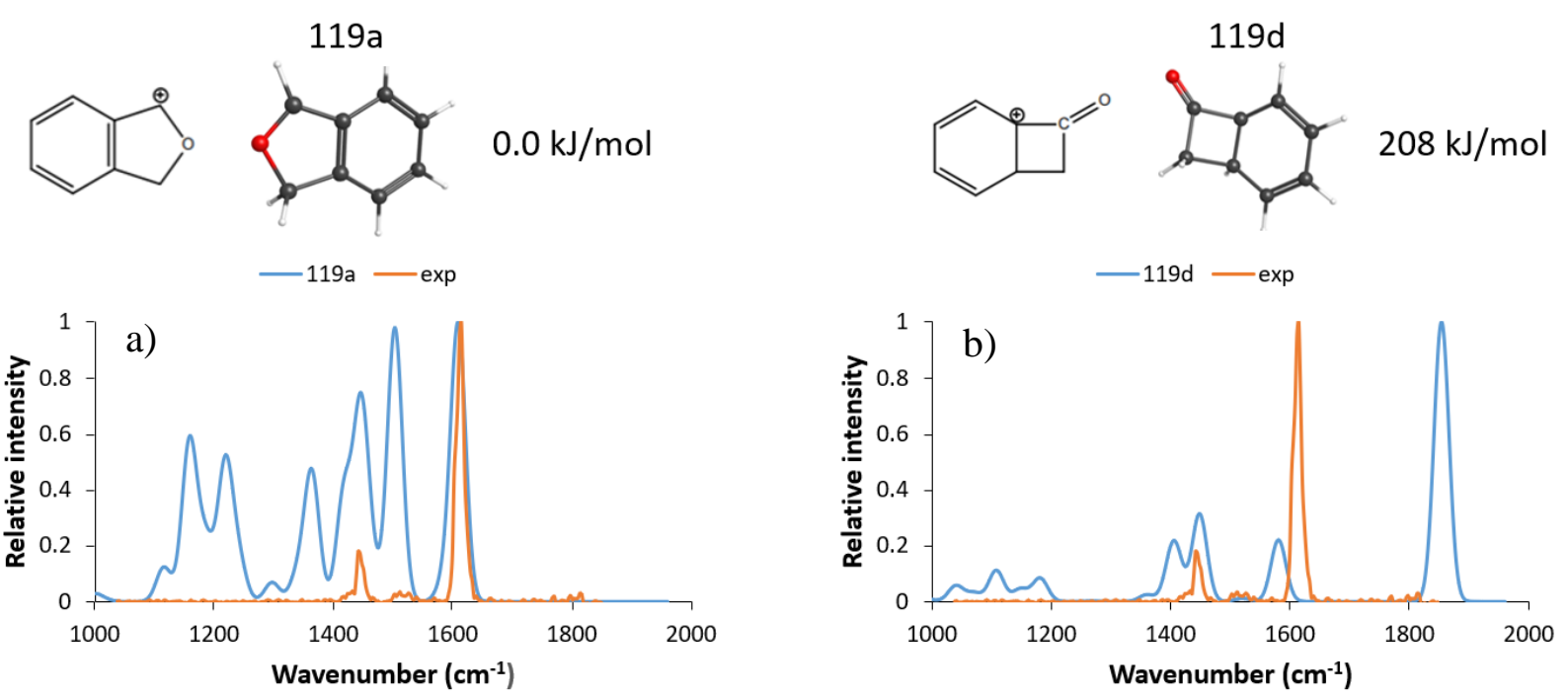

Figure 1.22. Comparison of experimental gas-phase IR ion spectroscopy and DFT theoretical IR spectra for fragments of protonated $\alpha$-PVP: a) proposed 119a structure demonstrating a good alignment between experimental and predicted spectra and b) proposed 119d structure demonstrating a poor alignment between the experimental and predicted spectra.

Figure 1.23 shows the analogous comparison for the intermediate product ion at $\mathrm{m} / \mathrm{z} .133$. The 133a' structure was identified as the most likely structure for the intermediate product ion at $\mathrm{m} / \mathrm{z}$ 133 based on the relative energy $(35 \mathrm{~kJ} / \mathrm{mol}$ more stable than the next isomer Table 1.2) and similarity between the experimental and theoretical spectra. The experimental band at $\sim 1600 \mathrm{~cm}^{-1}$ matches well with the theoretical absorption frequency for a highly-coupled $\mathrm{C}-\mathrm{C}$ stretching mode involving all aromatic carbons and $\mathrm{C}$ (aromatic) $-\mathrm{C} \alpha$ bonds. The experimental absorption at $\sim 1500$ $\mathrm{cm}^{-1}$ is matched by $\mathrm{C} \alpha-\mathrm{C} \beta$ and $\mathrm{C} \alpha-\mathrm{O}$ stretching coupled to aromatic $\mathrm{C}-\mathrm{H}$ wagging. The absorption at $\sim 1400 \mathrm{~cm}^{-1}$ is where $\mathrm{CH}_{2}$ and $\mathrm{CH}_{3}$ scissoring motions are predicted to absorb. In comparison, structure $133 \mathrm{c}$ gives a poorer fit because of the high relative energy $(123 \mathrm{~kJ} / \mathrm{mol})$ and the significant differences between the experimental and theoretical spectra. For instance, the main predicted band, the $\mathrm{C}=\mathrm{O}$ stretch coupled to adjacent $\mathrm{C}-\mathrm{C}$ stretching modes, should appear near $1700 \mathrm{~cm}^{-1}$, but is absent in the experimental IRMPD spectrum. Thus, the IR spectra indicate that neither an epoxide nor a carbonyl functional group are abundant in the ions at $m / z 119$ or $m / z 133$. 
The gas-phase IR spectra in Figure 1.23 indicates the presence of two structures in the IR spectra. The structure 133a' has both the best spectral match and the lowest energy configuration and is likely the dominant structure. However, the structure $133 \mathrm{~g}$, which lacks an oxygen, cannot be ruled out and is also likely to be present. The $\mathrm{MS}^{\mathrm{n}}$ and accurate mass data indicate the loss of the $\mathrm{CO}$ is favored over the loss of ethylene $\left(\mathrm{C}_{2} \mathrm{H}_{4}\right)$ for $\alpha$-PVP. However, the accurate mass data indicates that both pathways co-exist, so both structures are likely to be present in the gas-phase ion spectroscopy analysis. Due to the isobaric nature of these compounds, the isolation step before IR irradiation of the ions is unable to separate the intermediates $\mathrm{C}_{9} \mathrm{H}_{9} \mathrm{O}^{+}$and $\mathrm{C}_{10} \mathrm{H}_{13}{ }^{+}$that are both observed at nominal $\mathrm{m} / \mathrm{z} 133$.

133a'
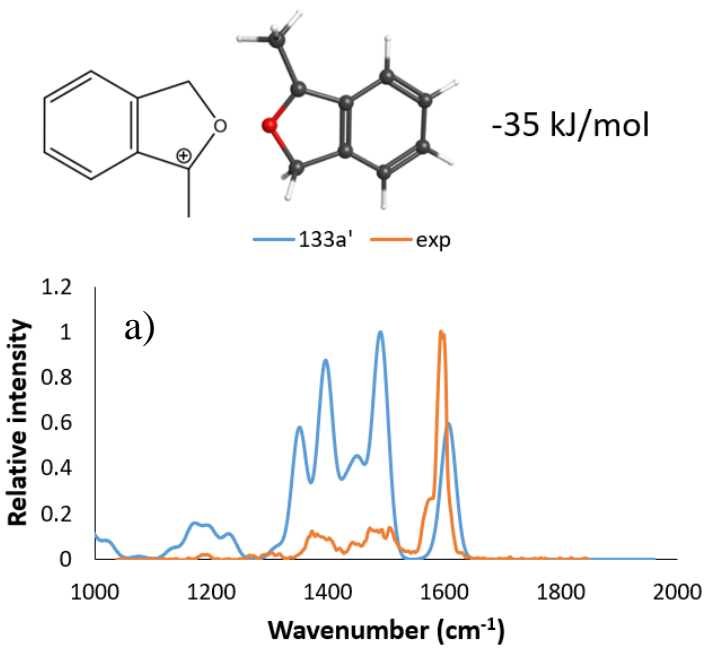

$133 c$
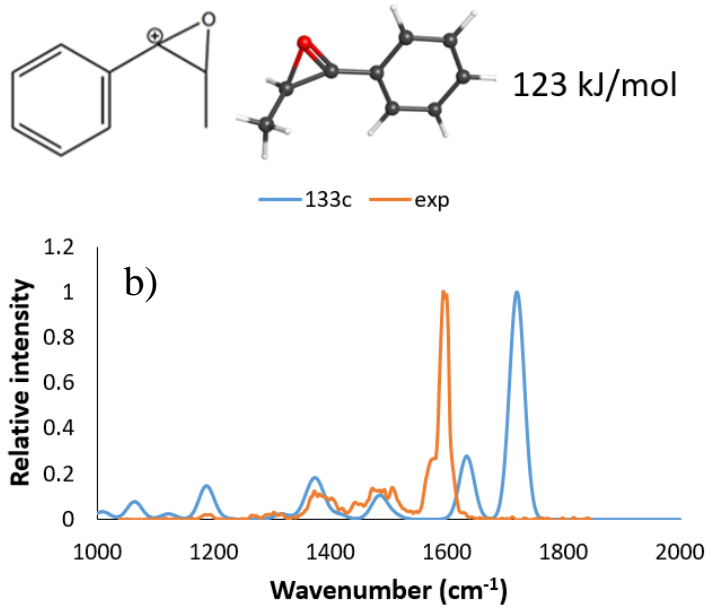

Figure 1.23. Comparison of experimental gas-phase IR ion spectroscopy and DFT calculated IR spectra for fragments of protonated $\alpha$-PVP: a) proposed 133a' structure demonstrating a good alignment between experimental and predicted spectra and b) proposed $133 \mathrm{c}$ structure demonstrating a poor alignment between the experimental and predicted spectra.

The various experiments described above enable the generation of a general set of rules to describe the fragmentation patterns and the formation of substituted tropylium ions in the tandem mass spectra of substituted cathinones. For example, the base peak in the tandem mass spectra of most $\alpha$-pyrrolidine-containing cathinones stems from the neutral loss of the pyrrolidine moiety. 
The only exception in our studies was 2,3-MDPV. The second common feature in the fragmentation behavior is the competition between the loss of $\mathrm{CO}$ and ethylene $\left(\mathrm{C}_{2} \mathrm{H}_{4}\right)$ from this base peak, which both have a nominal mass of $28 \mathrm{Da}$. Of the two losses, the loss of CO is typically dominant.

Another experimentally observed aspect of the fragmentation behavior is that competing fragmentation pathways lead to the formation of the tropylium ion via various neutral losses from the alkyl chain. As far as we have established, the fragmentation pathways seem to go through one of two phthalane structures shown in Figure 1.7; the only difference between the two is whether or not there is a methyl substitution on the saturated phthalane ring. The use of ${ }^{13} \mathrm{C}$ isotopic labeling on both the carbonyl carbon and $\alpha$-carbon demonstrate that the $\alpha$-carbon from the $m / z, 120$ intermediate ion is almost exclusively incorporated into the tropylium ion and the carbonyl carbon is quantitatively lost as neutral $\mathrm{CO}$, which, to our knowledge, has not been demonstrated in literature before.

There are several requirements for the proposed tropylium ion rearrangements to occur. First, the alkyl chain must be at least four carbon atoms long (including the carbonyl carbon) to enable the necessary rearrangements to occur. Second, there must be a reasonable leaving group available prior to ring expansion. Third, the $\alpha$-carbon must not be substituted; in the case of methyl substitution on the $\alpha$-carbon, for example, the methyl group poses a barrier to nucleophilic attack and the bicyclic phthalane intermediate cannot form. A final trend in tropylium ion behavior is that the abundance of the tropylium ion tends to increase with increasing alkyl chain lengths. Presumably, longer alkyl chains provide additional pathways towards the tropylium ion and larger alkyl chains provide more stable leaving groups. 
Regarding other trends in fragmentation behavior, the mechanisms described in Figure 1.7 remain conserved even with additional substituents. For example, the intermediate products ions of 3,4-MDPV show a similar distribution to $\alpha$-PVP, but shifted by the expected $44 \mathrm{Da}$ of the methylenedioxy substituent. Similarly, when a methyl group is present on the aromatic ring, such as with MPHP, the substituted tropylium ion at $m / z, 105\left(\mathrm{C}_{8} \mathrm{H}_{9}{ }^{+}\right)$is more abundant than the native/unsubstituted tropylium ion at $\mathrm{m} / \mathrm{z} 91$. However, for cathinones with different substituents

on the aromatic ring, the favorability of a specific pathway can be influenced by the position of the substituent, as demonstrated for 3,4-MDPV versus 2,3-MDPV. Finally, whereas the proposed mechanisms in Figure 1.7 do not pinpoint the exact hydrogens involved in the hydride shifts, the product ions at $m / z, 134$ and $m / z, 92$ of deuterated 3,4-MDPV-d8 specifically exclude the hydrogens on the pyrrolidine ring. In this class of cathinones, the pyrrolidine neutral is lost before any hydrogen rearrangements occur.

\subsection{Conclusions}

This manuscript describes the use of $\mathrm{MS}^{\mathrm{n}}$ on an IT mass spectrometer, accurate mass measurements with HRMS, stable isotope labeling, and gas-phase infrared ion spectroscopy to elucidate the mechanism of formation of the tropylium ion in the tandem mass spectra of various $\alpha$-pyrrolidinophenone synthetic cathinones. The identification of the proposed carbon backbone rearrangements for the generation of the tropylium ion or substituted tropylium ion analogs with ESI-MS/MS provides the forensic science community with new information about the fragmentation behavior of protonated synthetic cathinones. The conserved nature of the proposed mechanisms offers an additional tool for the identification of emerging synthetic cathinones using tandem mass spectra. Finally, the ability to defend the observations of existing casework and better predict the fragmentation patterns of future synthetic cathinones provides analysts with increased 
confidence in their interpretations and provides a stronger scientific foundation for their opinions in court. 


\section{Chapter 2: Fragmentation pathways of $\alpha$-pyrrolidinophenone synthetic cathinones and their application to the identification of emerging synthetic cathinone derivatives}

Reproduced in part with permission from J.T. Davidson, Z.J. Sasiene, Y. Abiedalla, J. DeRuiter, C.R. Clark, G.P. Jackson, International Journal of Mass Spectrometry, DOI:

10.1016/j.jjms.2020.116343.

\subsection{Introduction}

Synthetic cathinones are phenylalkylamine derivatives designed to mimic the effects of the natural chemical cathinone, the psychoactive component of the Catha edulis plant, commonly referred to as khat. [1]. Because of their stimulant-like pharmacological effects, cathinones belong to a larger class of drugs known as novel psychoactive substances (NPS). Synthetic cathinones are often marketed as "not for human consumption" or "bath salts" to avoid legislative restrictions that have been imposed to decrease the sale and distribution of these compounds $[\underline{2}, \underline{3}]$. Unfortunately, these labels also deceive users into believing the substances are safe, which has resulted in numerous intoxication-related deaths [53]. Reported symptoms of synthetic cathinone abuse include euphoria, hallucinations, psychosis, paranoia, agitation, violent behavior, tachycardia, acidosis, seizures and even death [53, 54]. In 2011, the Drug Enforcement Administration (DEA) recognized a growing trend in synthetic cathinone abuse and provisionally scheduled mephedrone, methylone, and 3,4-methylenedioxypyrovalerone (3,4-MDPV) as Schedule I controlled substances [55]. However, regulation of synthetic cathinones is difficult because the synthesis of new analogs only requires minor modifications to the generic chemical structure. The structural modifications allow the new analogs to avoid legal regulations, but potentially lead to more harmful substances entering the illicit drug market [1]. 
Whereas synthetic cathinones have become widely distributed designer drugs, the $\alpha$ pyrrolidinophenone derivatives stand out as one of the most abused designer drugs [7]. This class of compounds includes $\alpha$-pyrrolidinopentiophenone ( $\alpha$-PVP), 3,4-methylenedioxypyrovalerone (3,4-MDPV), $\alpha$-pyrrolidinoheptanophenone (PV8), $\alpha$-pyrrolidinopropiophenone ( $\alpha$-PPP), among others [56]. The first reported seizure of these $\alpha$-pyrrolidinophenone derivatives was in Germany in 1996 [57]. The key structural element of $\alpha$-pyrrolidinophenone derivatives is the pyrrolidine ring substitution to the generic synthetic cathinone structure [56]. The main types of $\alpha-$ pyrrolidinophenone derivatives are side chain extensions and substitution on the aromatic ring (methoxy and methylenedioxy are the most common) [56].

Synthetic cathinones are inhibitors of monoamine transporters such as the dopamine transporter (DAT) and the noradrenaline transporter (NAT), which is consistent with other stimulant compounds such as amphetamines. However, $\alpha$-pyrrolidinophenone derivatives are stronger inhibitors of these systems in comparison to non-pyrrolidinophenone synthetic cathinone derivatives increasing the effectiveness of these compounds [58]. In comparison to amphetamines, synthetic cathinones generally struggle to cross the blood-brain barrier, but the increased lipophilicity of the pyrrolidine substituent allows $\alpha$-pyrrolidinophenone derivatives to more easily cross the blood-brain barrier than other cathinones [56, 59].

Due to the widespread analysis of $\alpha$-pyrrolidinophenone synthetic cathinones, there is a need to understand the fragmentation behavior under different ionization and fragmentation conditions. The analysis of seized drugs typically employs gas chromatography-electron ionization-mass spectrometry (GC-EI-MS) to identify unknowns, whereas the toxicological community often employs liquid chromatography with electrospray ionization and tandem mass spectrometry (LCESI-MS/MS). A third form of ionization that is prevalent in national laboratories is direct analysis 
in real time (DART). Whereas DART produces both odd-electron and even-electron ions based on the experimental conditions, the fragment ion spectra from EI (odd-electron) and ESI (evenelectron) are known to differ, which has been documented for a variety of synthetic cathinones $[\underline{13-17}, \underline{19-21}, \underline{60]}$.

The fragmentation behavior of $\alpha$-pyrrolidinophenone synthetic cathinones has been reported throughout literature; however, rarely are the underlying fragmentation mechanisms that lead to the observed fragment ions discussed or understood [14, 16, 20, 26-29]. Instead, the mechanism(s) are either absent, vague, or improbable. For example, when valid mechanisms have been proposed, the lack of isotopic labeling and lack of multi-stage mass spectrometry $\left(\mathrm{MS}^{\mathrm{n}}\right)$ limit the certainty associated with the proposed mechanisms [30]. Recently, we demonstrated the first use of isotopic labeling, multi-stage mass spectrometry $\left(\mathrm{MS}^{\mathrm{n}}\right)$, accurate mass measurements with high-resolution mass spectrometry (HRMS) and ion spectroscopy to explain the formation of the tropylium ion $(m / z 91)$ and its substituted derivatives for $\alpha$-pyrrolidinophenone synthetic cathinones analyzed with ESI-MS/MS [61]. Here, we extend the study to a wider variety of $\alpha$-pyrrolidinophenone synthetic cathinone structures and include fragmentation pathways accessed through radical and even-electron pathways. The generation of synthetic cathinone fragmentation pathways will assist with the mass spectral interpretation and identification of future synthetic cathinone derivatives.

\subsection{Methods}

\subsubsection{Sample Preparation}

This study involved the analysis of $22 \alpha$-pyrrolidinophenone synthetic cathinones that were either purchased through Cayman Chemical (Ann Arbor, MI, USA) or synthesized in-house at Auburn University. The $11 \alpha$-pyrrolidinophenone synthetic cathinones that were purchased through Cayman Chemical were: $\alpha$-pyrrolidinopropiophenone $\quad(\alpha-P P P), \quad \alpha-$ 
pyrrolidinobutiophenone $\quad(\alpha-\mathrm{PBP}), \quad \alpha$-pyrrolidinovalerophenone $\quad(\alpha-\mathrm{PVP}), \quad \alpha-$ pyrrolidinoheptanophenone (PV8), 4-methoxy- $\alpha$-pyrrolidinopentiophenone (4-MeO- $\alpha$-PVP), 3',4'-trimethylene- $\alpha$-pyrrolidinovalerophenone, 3,4-methylenedioxy- $\alpha$-pyrrolidinopropiophenone (3,4-MDPPP), 3,4-methylenedioxy- $\alpha$-pyrrolidinobutiophenone $\quad$ (3,4-MDPBP), 3,4methylenedioxypyrovalerone $\quad(3,4-\mathrm{MDPV}), \quad 3,4$-methylenedioxypyrovalerone- $\mathrm{d}_{8} \quad$ on the pyrrolidine ring (3,4-MDPV- $\left.\mathrm{d}_{8}\right)$, and 2,3-methylenedioxypyrovalerone (2,3-MDPV). The 11 synthetic cathinone samples synthesized at Auburn University were: ${ }^{13} \mathrm{C}-\alpha-$ pyrrolidinovalerophenone labeled on the carbonyl carbon $\left({ }^{13} \mathrm{C}\right.$-carbonyl carbon- $\alpha$-PVP), ${ }^{13} \mathrm{C}-\alpha-$ pyrrolidinovalerophenone labeled on the $\alpha$-carbon $\quad\left({ }^{13} \mathrm{C}-\alpha\right.$-carbon- $\left.\alpha-\mathrm{PVP}\right), \quad{ }^{18} \mathrm{O}-\alpha-$ pyrrolidinovalerophenone $\left({ }^{18} \mathrm{O}-\alpha\right.$-PVP $), \alpha$-pyrrolidinovalerophenone- $\mathrm{d}_{7}$ labeled on the alkyl chain ( $\alpha$-PVP- $\left.\mathrm{d}_{7}\right), \alpha$-pyrrolidinovalerophenone- $\mathrm{d}_{8}$ labeled on the pyrrolidine ring $\left(\alpha-\mathrm{PVP}-\mathrm{d}_{8}\right), \alpha$-methylpyrrolidinovalerophenone ( $\alpha$-PVP-methyl group), ${ }^{13} \mathrm{C}$ - $\alpha$-pyrrolidinoheptanophenone labeled on the carbonyl carbon $\left({ }^{13} \mathrm{C}\right.$-carbonyl carbon-PV8), ${ }^{13} \mathrm{C}-\alpha$-pyrrolidinopropiophenone on the $\alpha$-carbon $\left({ }^{13} \mathrm{C}-\alpha\right.$-carbon- $\alpha$-PPP), ${ }^{13} \mathrm{C}-4^{\prime}$-methyl- $\alpha$-pyrrolidinohexanophenone on the carbonyl carbon $\left({ }^{13} \mathrm{C}\right.$ carbonyl carbon-MPHP), ${ }^{13} \mathrm{C}-3,4$-methylenedioxypyrovalerone on the carbonyl carbon $\left({ }^{13} \mathrm{C}\right.$ carbonyl carbon-3,4-MDPV), and ${ }^{13} \mathrm{C}-\mathrm{Naphyrone}$ on the carbonyl carbon. A full characterization of the synthetic samples was performed with GC-EI-MS and nuclear magnetic resonance spectroscopy (NMR) at Auburn University to confirm the correct labeling and acceptable purity prior to shipment to West Virginia University. All samples were analyzed at a concentration of approximately $100 \mathrm{ppm}$. The samples analyzed by GC-EI-MS were dissolved in HPLC grade methanol from Fisher Scientific (Palo Alto, CA, USA). All non-deuterated tandem MS samples analyzed on the Velos Pro linear ion trap (LIT) and the Accurate-Mass quadrupole time-of-flight mass spectrometer (Q-TOF) were dissolved in a solution of 49\% HPLC grade methanol, 49\% 
distilled water and $2 \%$ acetic acid. The acetic acid was supplied by Acros Organics (Palo Alto, CA, USA). The deuterated $\alpha$-pyrrolidinophenone synthetic cathinones were dissolved in HPLC grade methanol to minimize H/D exchange.

\subsubsection{Instrumentation}

\subsubsection{Linear Ion Trap}

The Thermo Scientific Velos Pro linear ion trap (LIT) mass spectrometer was mounted with a heated-electrospray ionization (HESI) source. The HESI source was operated at $50{ }^{\circ} \mathrm{C}$ with a spray voltage of $4,000 \mathrm{~V}$. The nitrogen sheath gas was operated at 8 arbitrary units with a nitrogen auxiliary gas flow of 5 arbitrary units. The mass spectrometer capillary temperature was $275^{\circ} \mathrm{C}$. The scan range and normalized collision energies (NCE), which were optimized for each compound are labeled with each mass spectrum. The \% NCEs are sequential in that the provided $\% \mathrm{NCE}$ at the $\mathrm{MS}^{2}$ level is used to reach the $\mathrm{MS}^{3}$ level prior to the $\% \mathrm{NCE}$ used for fragmentation at the $\mathrm{MS}^{3}$ level.Ultra-high purity helium was used as the bath gas purchased through Matheson TRIGAS (Fairmont, WV, USA).

\subsubsection{Quadrupole Time-of-Flight}

An Agilent Technologies 6538 UHD Accurate-Mass quadrupole time-of-flight (Q-TOF) mass spectrometer was operated with both dual ESI and DART ionization sources. The DART-100 source was mounted with a Vapur® interface (IonSense, Saugus, MA, USA). The DART ion source was operated with helium reagent gas at $300{ }^{\circ} \mathrm{C}$ with a flow rate of $3.0 \mathrm{~L} / \mathrm{min}$, a grid voltage of $400 \mathrm{~V}$ and a needle voltage of 3,500 V. The ESI source was operated with a spray voltage of $3,500 \mathrm{~V}$, nitrogen drying gas at a $300{ }^{\circ} \mathrm{C}$ and a flow rate of $5 \mathrm{~L} / \mathrm{min}$. The nebulizer flow was 30 psig. All ESI samples were collected with direct injection at a flow rate of $10 \mu \mathrm{L} / \mathrm{min}$. The MS fragmentor and skimmer voltages were held at $225 \mathrm{~V}$ and $65 \mathrm{~V}$, respectively for the ESI data 
collection and $150 \mathrm{~V}$ and $25 \mathrm{~V}$, respectively for the DART data collection. The DART data collection used lower fragmentor and skimmer voltages per the recommendations of IonSense for optimum performance. For both ion sources, the fragmentor voltage and skimmer voltage relationship was optimized to maximize the $[\mathrm{M}+\mathrm{H}]^{+}$protonated precursor, with disregard to insource fragmentation due to the isolation of the protonated precursor prior to collisional activation. The scan range and collision energies were optimized for each compound of interest and are labeled with each mass spectrum. An isolation width of 1.3 Da was used for all samples. The ultrahigh purity nitrogen used for the collision gas and the ultra-high purity helium used for the DART gas were purchased through Matheson TRIGAS (Fairmont, WV, USA).

The DART samples were prepared through the deposition of $5 \mu \mathrm{L}$ of drug standard onto the closed end of $1.5 \mathrm{~mm}$ x $1.8 \mathrm{~mm}$ x $90 \mathrm{~mm}$ Pyrex® glass capillaries purchased from Corning Life Sciences (Corning, New York, USA) and allowed to completely dry before analysis. DART acquisition consisted of approximately $30 \mathrm{~s}$ of background collection, about $5 \mathrm{~s}$ of sample introduction, and then the analysis of a blank capillary to account for capillary-specific background. The total length of analysis was less than $90 \mathrm{~s}$ per sample.

\subsubsection{Single Quadrupole GC-EI-MS}

An Agilent Technologies 7890 GC-5977 MS with an HP-5 [(5\% phenyl)-methylpolysiloxane] $30 \mathrm{~m}$ x $250 \mu \mathrm{m}$ x $0.25 \mu \mathrm{m}$ column by Agilent J\&W Columns was used for the GC-EI-MS analyses. The GC-EI-MS parameters were as follows: injection volume was $1 \mu \mathrm{L}$; injection temperature was $250{ }^{\circ} \mathrm{C}$; split ratio was $20: 1$. The initial oven temperature was $80{ }^{\circ} \mathrm{C}$ ( 1 min hold), which was ramped to $280{ }^{\circ} \mathrm{C}$ at $15^{\circ} \mathrm{C} / \mathrm{min}$, then held for $2 \mathrm{~min}$. The carrier gas (helium) flow rate was set to $1 \mathrm{~mL} / \mathrm{min}$ and the transfer line temperature was set to $280{ }^{\circ} \mathrm{C}$. The ultra-high purity helium gas was purchased from Matheson TRIGAS (Fairmont, WV, USA). The mass spectrometer was 
scanned from $\mathrm{m} / \mathrm{z}, 50-500$ at a scan rate of 1,500 Da/sec after a solvent delay of $2 \mathrm{~min}$. The source and quadrupole temperatures were $250{ }^{\circ} \mathrm{C}$ and $200{ }^{\circ} \mathrm{C}$, respectively.

\subsubsection{Data Analysis}

Xcalibur 2.0.0.48 software was used for the data analysis on the Velos Pro. ChemStation version C.01.01 was used for the Agilent GC-EI-MS data analysis and MassHunter Qualitative Analysis B.05.00 was used for the Agilent Q-TOF data analysis. Microsoft Excel version 14 (Microsoft, Redmond, WA, USA) and ChemDraw 16.0 (PerkinElmer, Waltham, MA, USA) were used for mass spectral plots and mass spectral fragmentation mechanisms.

\subsubsection{Mass Spectral Interpretation and Mechanisms}

The proposed fragmentation pathways follow the expected lowest energy structures and are based on rational electron pushing mechanisms commonly used for the interpretation of protonated tandem MS and EI-MS data [35]. The use of isotopic labels, $\mathrm{MS}^{\mathrm{n}}$ and HRMS allows for the structural determination of all intermediates along the proposed fragmentation pathways. Deuterium labeling is not always able to identify the specific deuterium atoms and hydrogen atoms involved in tandem MS rearrangements, but such labeling usually offers some insight into the general fragmentation behavior. Finally, the observation of protonated precursor ions (evenelectron) forming odd-electron product ions was observed along minor abundance pathways, which is consistent with previous reports from Fornal [흐, $\underline{37]}$.

\subsection{Results/Discussion}

\subsubsection{HESI-Velos Pro MS}

Our previous work with $\alpha$-pyrrolidinophenone synthetic cathinones involved the identification that the tropylium ion $(\mathrm{m} / \mathrm{z}$ 91) or substituted derivative ions form through different oxygencontaining intermediates that exclusively retain the $\alpha$-carbon with the corresponding loss of the 
carbonyl carbon as neutral $\mathrm{CO}$ [61]. Through this project we also discovered several fragmentation behaviors of the $\alpha$-pyrrolidinophenone class of synthetic cathinones. First, we identified competitive pathways for the loss of $\mathrm{CO}$ and ethylene $\left(\mathrm{C}_{2} \mathrm{H}_{4}\right)$ from the base peak of the tandem mass spectrum. Second, we determined that the base peak in the tandem mass spectrum is primarily formed through the loss of the neutral pyrrolidine molecule. Third, we identified that the alkyl chain length has a direct impact on not only the tropylium ion formation, but also the associated intermediate product ions. Finally, we demonstrated that the $\alpha$-pyrrolidinophenone synthetic cathinone fragmentation pathways remain conserved when accounting for additional substituents.

Figure 2.1a shows the $\mathrm{MS}^{2}$ analysis of protonated $\alpha-\mathrm{PVP}-\mathrm{d}_{8}$, which is deuterated around the pyrrolidine ring. The product ion spectrum indicates that the deuterium labels remain on the cleaved 1-butylidenepyrrolidin-1-ium fragment observed at $\mathrm{m} / \mathrm{z} 134$ and that no H/D scrambling occurs prior to fragmentation. The lone exception to this observation is the presence of the primary product ion at $\mathrm{m} / \mathrm{z} 221$, which must arise through the loss of $\mathrm{HDO}$ instead of $\mathrm{H}_{2} \mathrm{O}$, the latter of which occurs to a slightly lesser extent at $m / 2$ 222. As expected, the $\mathrm{MS}^{3}$ product ion spectrum for the pathway $m / z, 240 \rightarrow 161 \rightarrow($ Figure 2.1b) is consistent with structures proposed for the analysis of $\alpha$-PVP [61], with the $m / z 161$ intermediate product ion formed through the loss of the pyrrolidine moiety. Figure 2.1c shows the $\mathrm{MS}^{3}$ spectrum for the pathway $m / z, 240 \rightarrow 134 \rightarrow$, which results in secondary product ions at $\mathrm{m} / \mathrm{z}, 106,105$ and 92 . The intermediate at $\mathrm{m} / \mathrm{z}, 134$ likely has the structure of 1-butylidenepyrrolidin-1-ium and fragments through the loss of ethylene, an ethyl radical $\left({ }^{\circ} \mathrm{C}_{2} \mathrm{H}_{5}\right.$ ), and propylene to form the product ions at $\mathrm{m} / \mathrm{z}, 106,105$, and 92 , respectively. 

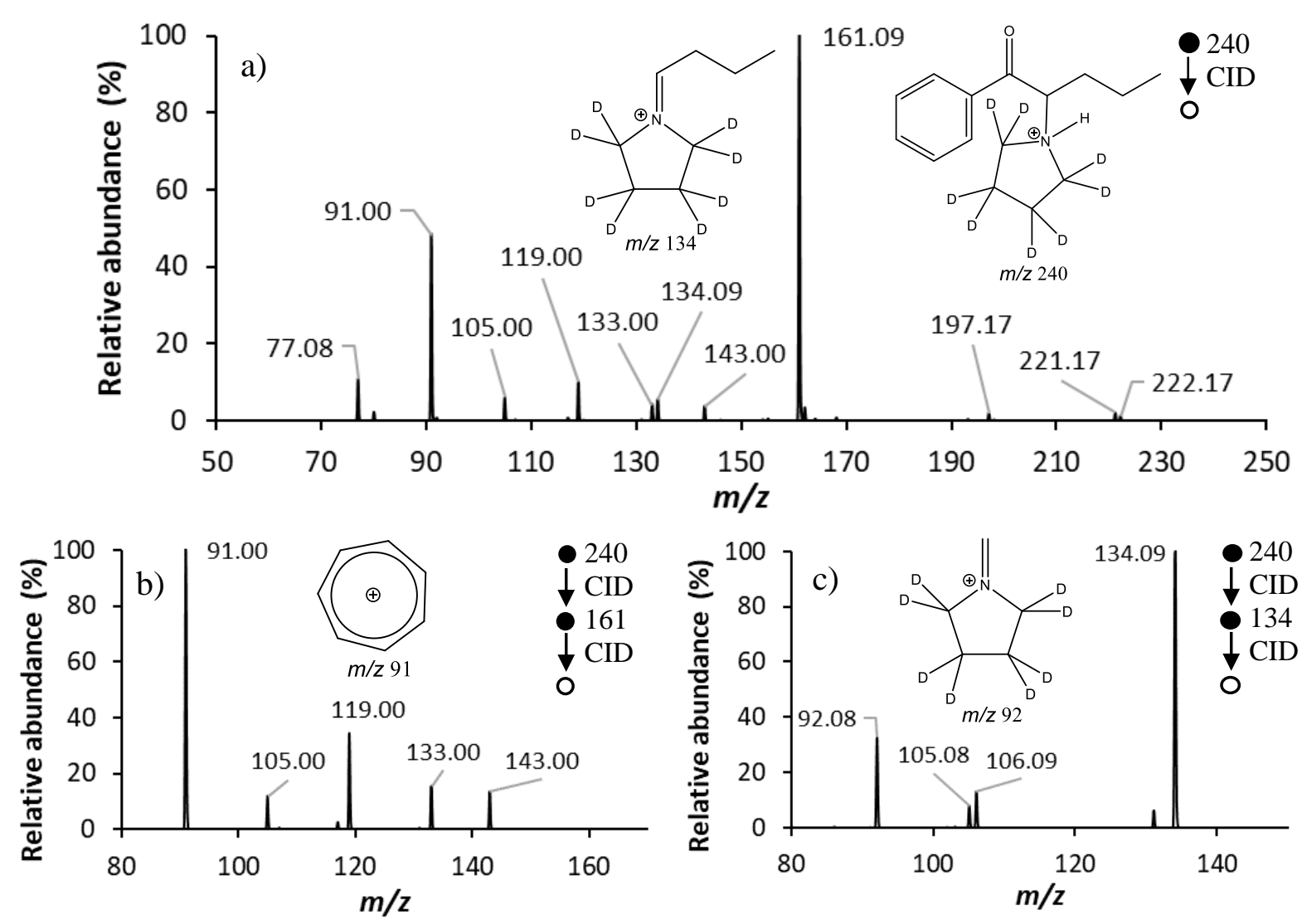

Figure 2.1. Tandem mass spectra of $\alpha-P V P-d_{8}$ : a) $\mathrm{MS}^{2}$ product ion spectrum of the $[\mathrm{M}+\mathrm{H}]^{+}$ molecular ion at $\mathrm{m} / \mathrm{z} 240(35 \% \mathrm{NCE})$; b) $\mathrm{MS}^{3}$ product ion spectrum for the pathway $\mathrm{m} / \mathrm{z}$ $240 \rightarrow 161 \rightarrow$ at $30 \% \mathrm{NCE}$ showing the formation of secondary product ions at $\mathrm{m} / \mathrm{z} 143,133$, 119,105 and 91 ; c) $\mathrm{MS}^{3}$ product ion spectrum for the pathway $m / z 240 \rightarrow 134 \rightarrow$ at $30 \% \mathrm{NCE}$ showing the formation of product ions at $\mathrm{m} / \mathrm{z}, 106,105$ and 92.

\subsubsection{DART/ESI-Q-TOF}

Figure 2.2 compares $\mathrm{MS}^{2}$ analysis of $4-\mathrm{MeO}-\alpha-\mathrm{PVP}$ collected using the ESI and DART ionization sources on the same HRMS instrument. The main benefit of the HRMS instrument is the ability to determine the elemental formula of the different fragment ions. The ESI and DART mass spectra are very similar in the position and abundance of fragments, and ESI shows primary product ions at $m / z 219.1282$ (expected at $\mathrm{m} / z, 219.1259$ for $\mathrm{C}_{13} \mathrm{H}_{17} \mathrm{O}_{2} \mathrm{~N}^{+\bullet} ; 10 \mathrm{ppm}$ error), $\mathrm{m} / z$ 191.1102 (expected at $m / z 191.1072$ for $\mathrm{C}_{12} \mathrm{H}_{15} \mathrm{O}_{2}{ }^{+} ; 16$ ppm error) and $m / z$ 154.1249 (expected at $m / z 154.1231$ for $\mathrm{C}_{9} \mathrm{H}_{16} \mathrm{ON}^{+} ; 12$ ppm error). Secondary product ions at $m / z, 135.0471$ (expected at $m / z$ 135.0446 $\mathrm{C}_{8} \mathrm{H}_{7} \mathrm{O}_{2}{ }^{+} ; 19$ ppm error) and $m / z, 126.1302$ (expected at $m / z, 126.1282$ for $\mathrm{C}_{8} \mathrm{H}_{16} \mathrm{~N}^{+}$; 
16 ppm error) are observed for ESI, and they are formed via the stepwise loss of $\mathrm{C}_{4} \mathrm{H}_{8}$ and CO from their primary product ions at $m / z, 191.1102\left(\mathrm{C}_{12} \mathrm{H}_{15} \mathrm{O}_{2}{ }^{+}\right)$and $m / z$ 154.1249 $\left(\mathrm{C}_{9} \mathrm{H}_{16} \mathrm{ON}^{+}\right)$, respectively. The tertiary product ion at $m / z, 121.0677$ (expected at $m / z, 121.0653$ for $\mathrm{C}_{8} \mathrm{H}_{9} \mathrm{O}^{+} ; 20$ ppm error) is the base peak in the ESI-generated mass spectrum. The fragment at $\mathrm{m} / \mathrm{z} 121.0677$ $\left(\mathrm{C}_{8} \mathrm{H}_{9} \mathrm{O}^{+}\right)$in the ESI-generated mass spectrum arises from the loss of $\mathrm{CO}$ from the secondary product ion at $m / z 149.0632$ (expected at $m / z, 149.0602$ for $\mathrm{C}_{9} \mathrm{H}_{9} \mathrm{O}_{2}{ }^{+} ; 20$ ppm error) and the loss of propylene from the secondary product ion at $m / z 163.1168$ (expected at $m / z \quad 163.1122$ for $\mathrm{C}_{11} \mathrm{H}_{15} \mathrm{O}^{+} ; 28$ ppm error). In all cases, the mass accuracy of both the ESI and DART mass spectra are on the order of $10 \mathrm{ppm}$ from the exact masses for the proposed elemental formulas, which provides a high degree of confidence in the elemental composition of the proposed product ion structures. 

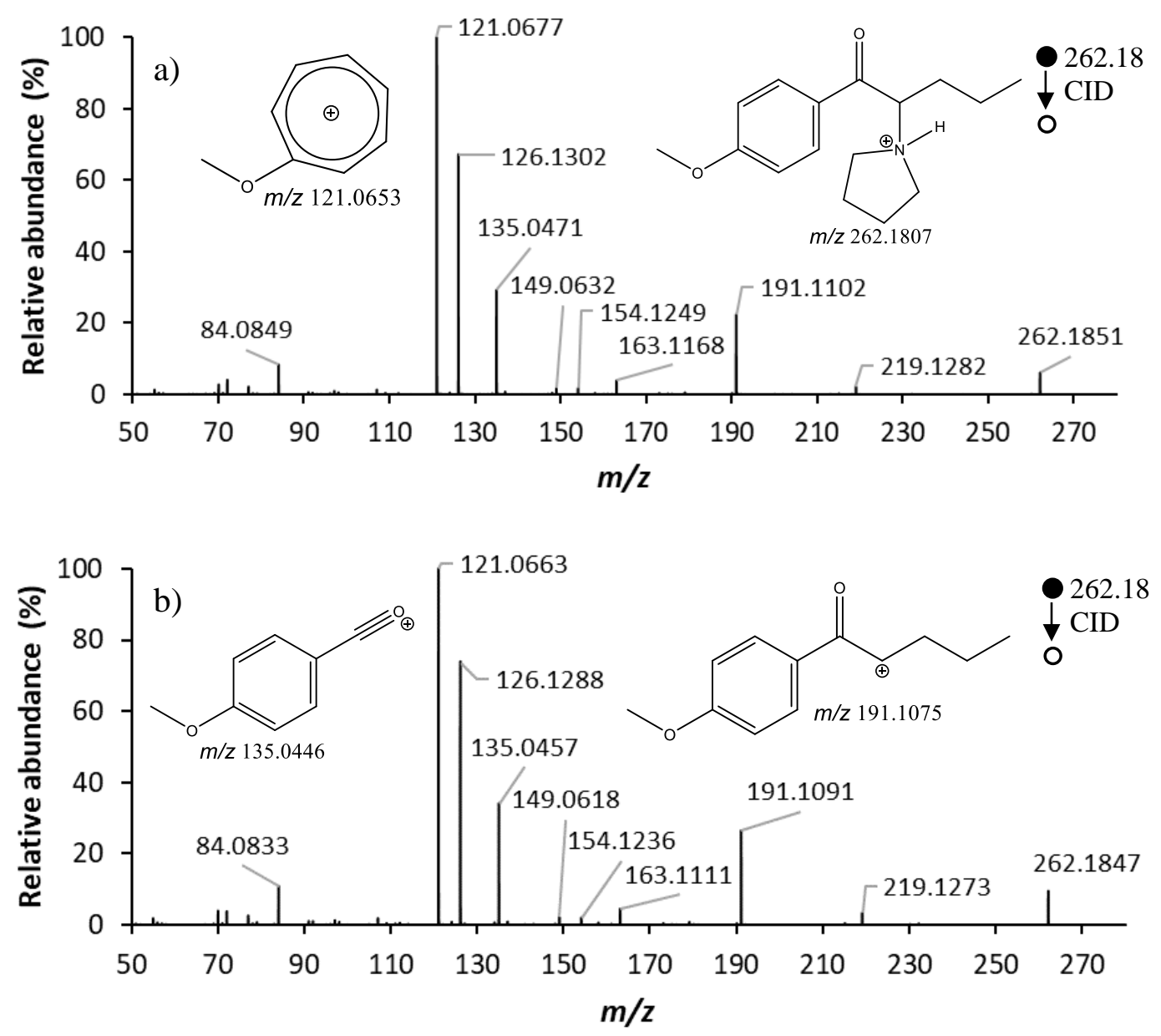

Figure 2.2. Tandem mass spectra of 4-MeO- $\alpha$-PVP collected on the same Q-TOF HRMS instrument using a) ESI with a $25 \mathrm{eV}$ collision energy and b) DART ionization with a $25 \mathrm{eV}$ collision energy.

Figure 2.3 highlights the similarities between the $\mathrm{MS}^{2}$ spectra from ESI and DART ionization of protonated 3,4-MDPV at $\mathrm{m} / 2,276.1607$ (expected at $\mathrm{m} / z 276.1599$ for $\mathrm{C}_{16} \mathrm{H}_{22} \mathrm{NO}_{3}{ }^{+} ; 3$ ppm error) for the ESI-generated mass spectrum. The most abundant ions appear at $\mathrm{m} / z 126.1305$ (expected at $m / z 126.1282$ for $\mathrm{C}_{8} \mathrm{H}_{16} \mathrm{~N}^{+} ; 18$ ppm error) and $m / z 135.0476$ (expected at $m / z 135.0446$ for $\mathrm{C}_{8} \mathrm{H}_{7} \mathrm{O}_{2}{ }^{+} ; 22 \mathrm{ppm}$ error) for the ESI-generated mass spectrum, which correspond to the 1butylidenepyrrolidin-1-ium ion and methylenedioxy-substituted tropylium ion, respectively. Due to the methylenedioxy substituent on the aromatic ring moiety, an additional dominant pathway is observed through the loss of formaldehyde $\left(\mathrm{CH}_{2} \mathrm{O}\right)$ from fragments containing the methylenedioxy 
group. Ions corresponding to the loss of formaldehyde are observed at $\mathrm{m} / \mathrm{z} 175.0812$ (expected at $m / z 175.0759$ for $\mathrm{C}_{11} \mathrm{H}_{11} \mathrm{O}_{2}+30$ ppm error) and $m / z 147.0821$ (expected at $m / z 147.0809$ for $\mathrm{C}_{10} \mathrm{H}_{11} \mathrm{O}^{+} ; 8$ ppm error) for the ESI-generated mass spectrum, which are both 30 Da less than their predecessor ions at $\mathrm{m} / \mathrm{z} 205.0922$ (expected at $\mathrm{m} / \mathrm{z} 205.0864$ for $\mathrm{C}_{12} \mathrm{H}_{13} \mathrm{O}_{3}{ }^{+} ; 28$ ppm error) and $m / z 177.0955$ (expected at $\mathrm{m} / z 177.0915$ for $\mathrm{C}_{11} \mathrm{H}_{13} \mathrm{O}_{2}{ }^{+} ; 23 \mathrm{ppm}$ error), respectively. Generally, the accurate mass measurements were on the same order of magnitude from the exact masses of the proposed elemental formula as those in Figure 2.2; however, there was less agreement in the accurate mass measurements between the ESI and DART data likely due to differences in the recency of the tune prior to each analysis. 

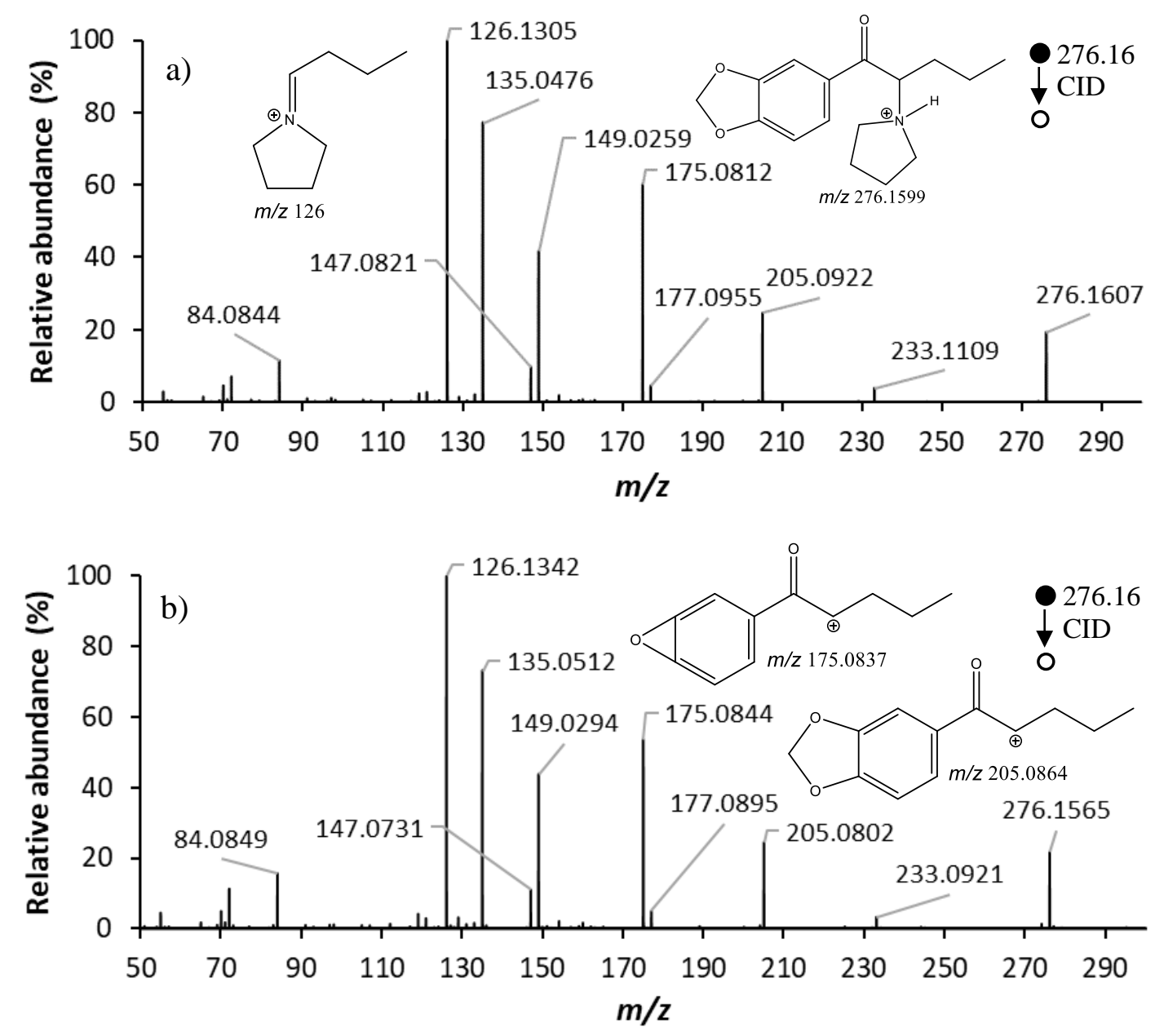

Figure 2.3. Tandem mass spectra of 3,4-MDPV collected on the same Q-TOF HRMS instrument using a) ESI with a $25 \mathrm{eV}$ collision energy and b) DART ionization with a $25 \mathrm{eV}$ collision energy.

Figure 2.4 compares the MS ${ }^{2}$ spectra of protonated adducts of ${ }^{13} \mathrm{C}$-MPHP formed by ESI and DART ionization. The labeled ${ }^{13} \mathrm{C}$ is on the carbonyl carbon in both cases. The spectra have the major structural fragments embedded. These spectra demonstrate that both ion sources produce similar mass spectra with the base peak observed at $\mathrm{m} / z 105.0727$ (expected at $\mathrm{m} / \mathrm{z} 105.0704$ for $\mathrm{C}_{8} \mathrm{H}_{9}{ }^{+} ; 22$ ppm error) for the ESI-generated mass spectrum. The formation of this ion is unexpected in that the isotopically labeled carbonyl adjacent to the aromatic ring moiety must be lost prior to ring expansion, which has been previously demonstrated as a possible fragmentation mechanism of $\alpha$-pyrrolidinophenone synthetic cathinones[61]. The secondary product ions 
at $m / z 204.1355$ (expected at $m / z \quad 204.1338$ for $\quad \mathrm{C}_{12}{ }^{13} \mathrm{CH}_{17} \mathrm{ON}^{+*} ; 8$ ppm error), $m / z$ 190.1315 (expected at $m / z, 190.1307$ for $\mathrm{C}_{12}{ }^{13} \mathrm{CH}_{17} \mathrm{O}^{+} ; 4$ ppm error), and $\mathrm{m} / \mathrm{z}, 140.14535$ (expected at $m / z 140.1439$ for $\mathrm{C}_{9} \mathrm{H}_{18} \mathrm{~N}^{+} ; 10$ ppm error) for the ESI-generated mass spectrum originate through the loss of a butyl radical $\left({ }^{\circ} \mathrm{C}_{4} \mathrm{H}_{9}\right)$, loss of the pyrrolidine molecule, and the generation of the 1pentylidenepyrrolidin-1-ium ion, respectively. The tertiary product ions at $\mathrm{m} / \mathrm{z} 134.0725$ (expected at $m / z 134.0681$ for $\mathrm{C}_{8}{ }^{13} \mathrm{CH}_{9} \mathrm{O}^{+} ; 33 \mathrm{ppm}$ error) and $\mathrm{m} / z, 120.0540$ (expected at $\mathrm{m} / z .120 .0524$ for $\mathrm{C}_{7}{ }^{13} \mathrm{CH}_{7} \mathrm{O}^{+} ; 13 \mathrm{ppm}$ error) for the ESI -generated mass spectrum form through the loss of butylene and stepwise loss of $\mathrm{C}_{5} \mathrm{H}_{10}$ from the primary product ion at $m / z 190.1315\left(\mathrm{C}_{12}{ }^{13} \mathrm{CH}_{17} \mathrm{O}^{+}\right)$. All accurate mass measurements are on the order of $10 \mathrm{ppm}$ different than the exact masses for the proposed elemental formulas, which was relatively consistent throughout the Q-TOF dataset. 

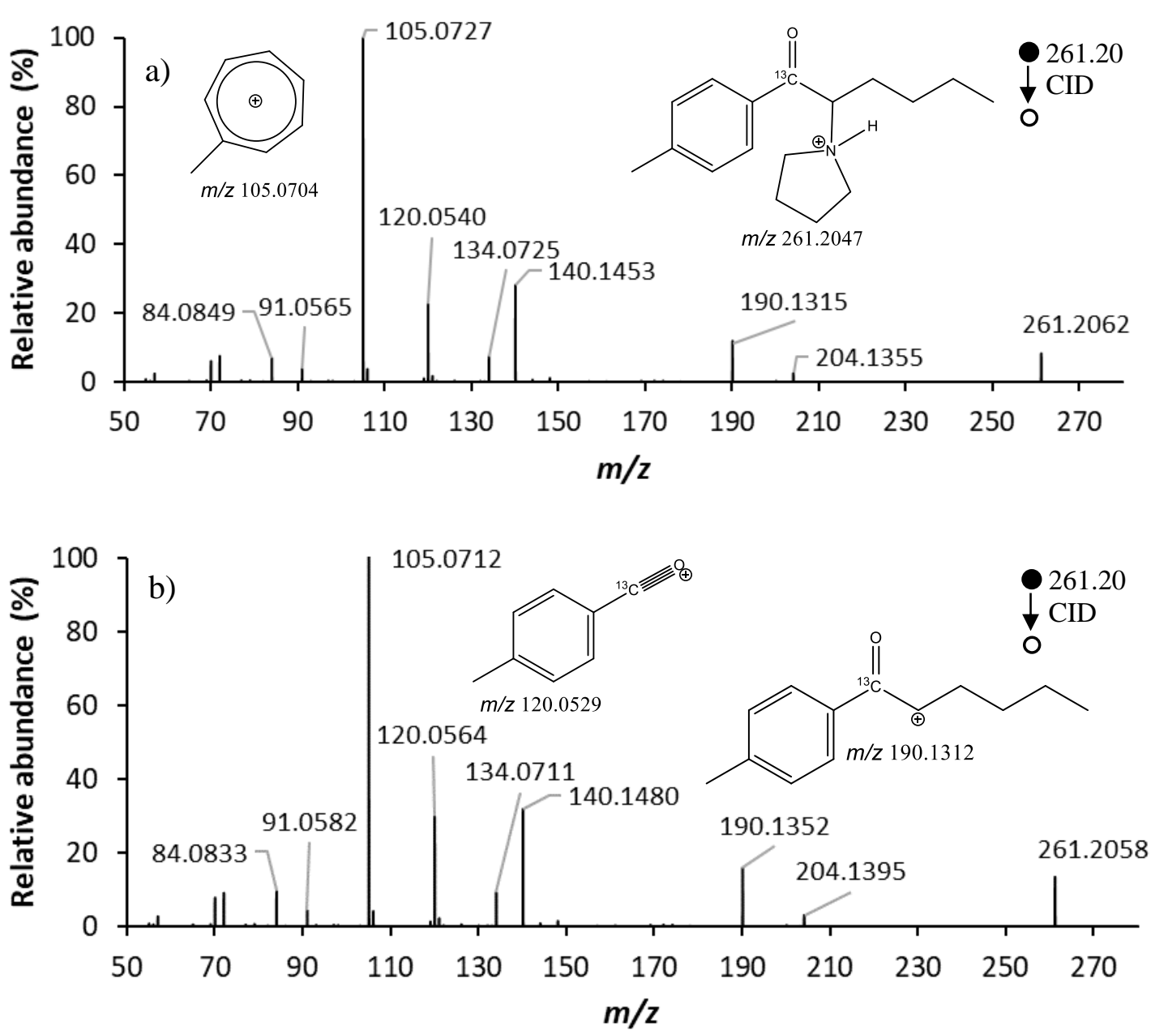

Figure 2.4. Tandem mass spectra of ${ }^{13} \mathrm{C}-\mathrm{MPHP}$ collected on the same Q-TOF HRMS instrument using a) ESI with a $25 \mathrm{eV}$ collision energy and b) DART ionization with a $25 \mathrm{eV}$ collision energy.

Figure 2.5 demonstrates the proposed general pathways for the fragmentation of $\alpha$ pyrrolidinophenone synthetic cathinones under protonated tandem MS conditions, where X represents substitution to the aromatic ring moiety and $\mathrm{C}_{n} \mathrm{H}_{2 n+1}$ represents varying alkyl chain lengths. The ${ }^{13} \mathrm{C},{ }^{18} \mathrm{O}$, and deuterated labeling are not shown in these general pathways but were used to support the proposed pathways. The proposed fragmentation pathways are based on data collected with both the IT and Q-TOF mass spectrometers, which are known to have differences in their fragmentation energy deposition rates. The fragmentation process with an IT mass 
spectrometer is considered very slow activation that occurs through 100s of collisions with the bath gas. In comparison, the Q-TOF fragmentation arises through low-energy, beam-type activation, involving 10s of collisions as the analyte passes through the collision cell [ $[\underline{51}, \underline{52]}$. However, in general, the mass spectra collected with both the IT and Q-TOF mass spectrometers behaved as described below in Figure 2.5 with the favorability of each fragmentation pathway observed under LIT conditions indicated by the size and color of the corresponding arrow. 


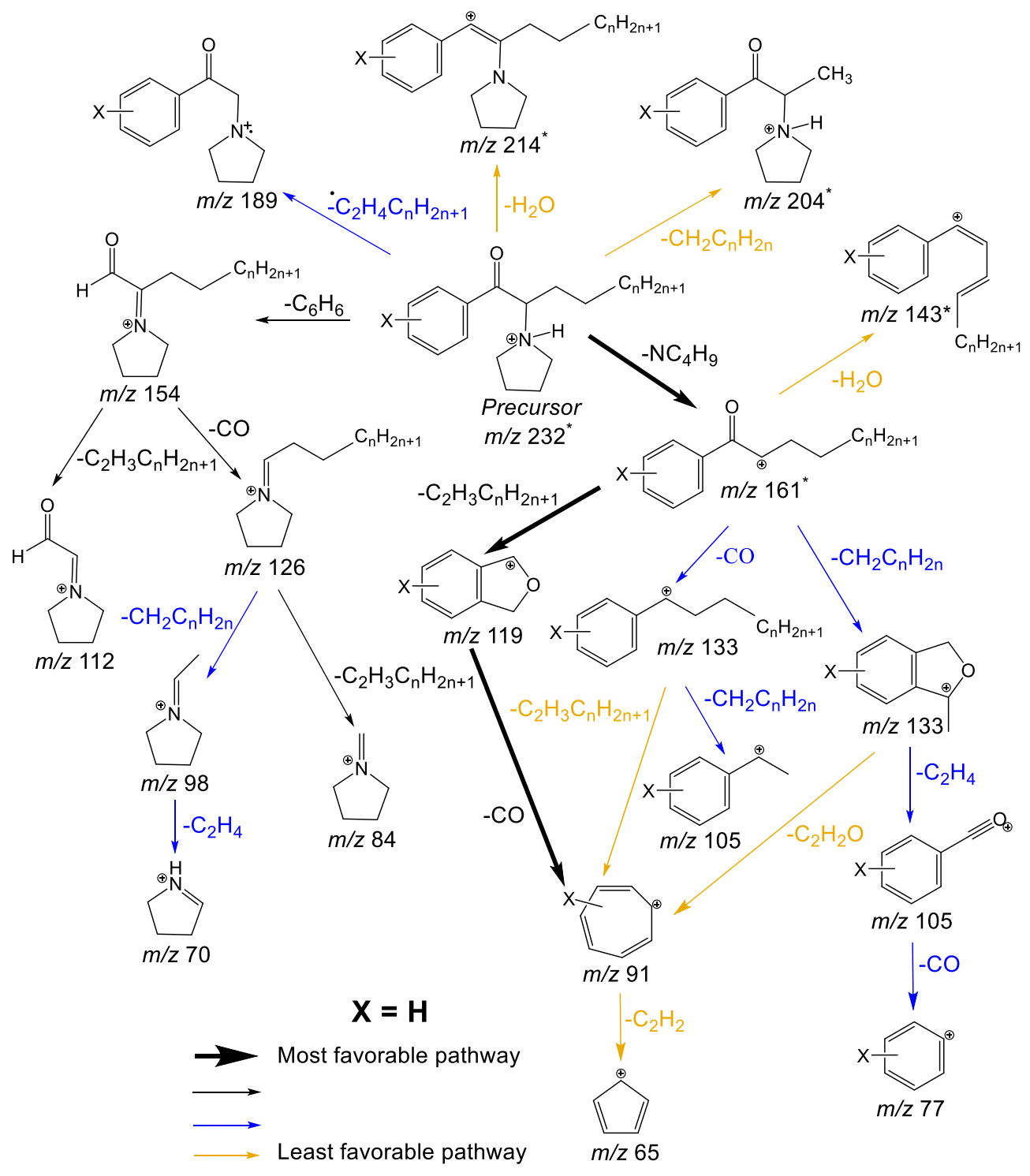

Figure 2.5. Proposed general fragmentation pathways for protonated $\alpha$-pyrrolidinophenone synthetic cathinones undergoing tandem MS. The model compound is $\alpha$-PVP where $X=H$ and the $m / z$ values that are specific to $\alpha$-PVP indicated with an asterisk (*).

Based on the analysis of $21 \alpha$-pyrrolidinophenone synthetic cathinones using isotope labeling, $\mathrm{MS}^{\mathrm{n}}$ and HRMS, the following general trends are observed. Isolation and fragmentation of the $[\mathrm{M}+\mathrm{H}]^{+}$precursor ion typically results in primary product ions through the loss of $\mathrm{CH}_{2} \mathrm{C}_{\mathrm{n}} \mathrm{H}_{2 \mathrm{n}}, \mathrm{H}_{2} \mathrm{O}$, ${ }^{\cdot} \mathrm{C}_{2} \mathrm{H}_{4} \mathrm{C}_{\mathrm{n}} \mathrm{H}_{\mathrm{n} 2+1}, \mathrm{C}_{6} \mathrm{H}_{6}$, and $\mathrm{NC}_{4} \mathrm{H}_{9}$. Of these primary product ions, the loss of $\mathrm{NC}_{4} \mathrm{H}_{9}$ (pyrrolidine molecule) is dominant, and the loss of $\mathrm{C}_{n} \mathrm{H}_{2 n}$ and $\mathrm{H}_{2} \mathrm{O}$ are the least prevalent fragmentation 
pathways. The two primary product ions that produce abundant consecutive product ions are the ions at $\mathrm{m} / \mathrm{z} 161$, from the loss of the pyrrolidine, and $\mathrm{m} / \mathrm{z} 154$, from the loss of the aromatic ring, in Figure 2.5. The fragmentation pathway through the intermediate at $m / z 154$ results in secondary product ions through the loss of CO (i.e. $m / z$ 126) and propylene (i.e. $m / z$ 112), which can then form tertiary and quaternary product ions. The fragmentation pathway through the intermediate at $m / z, 161$ in Figure 2.5 continues through three abundant secondary product ions at $m / z, 143,133$, and 119. The secondary product ions at $\mathrm{m} / \mathrm{z}, 143$ and $\mathrm{m} / \mathrm{z}, 119$ form through the loss of $\mathrm{H}_{2} \mathrm{O}$ and propylene, respectively. However, secondary product ions at $m / z 133$ form via competing pathways through the loss of $\mathrm{CO}(28 \mathrm{Da})$ and ethylene (28 Da), as demonstrated previously [61]. The secondary product ions at $m / z, 133$ in Figure 2.5 fragment into tertiary product ions at $m / z 105$ and $\mathrm{m} / \mathrm{z}$ 91. The secondary product ion at $\mathrm{m} / \mathrm{z} 119$ also fragment into the characteristic tropylium ion at $m / z$ 91, which helps explain the significant presence of the tropylium ion in $\alpha$ pyrrolidinophenone synthetic cathinones that contain at least four carbon atoms in the alkyl chain [61]. Figure 2.6 demonstrates the formation of the tropylium ion from $\alpha$-PBP, $\alpha$-PVP, and PV8 and the effect of alkyl chain lengths on the distribution of product ions observed in the protonated tandem mass spectra. 

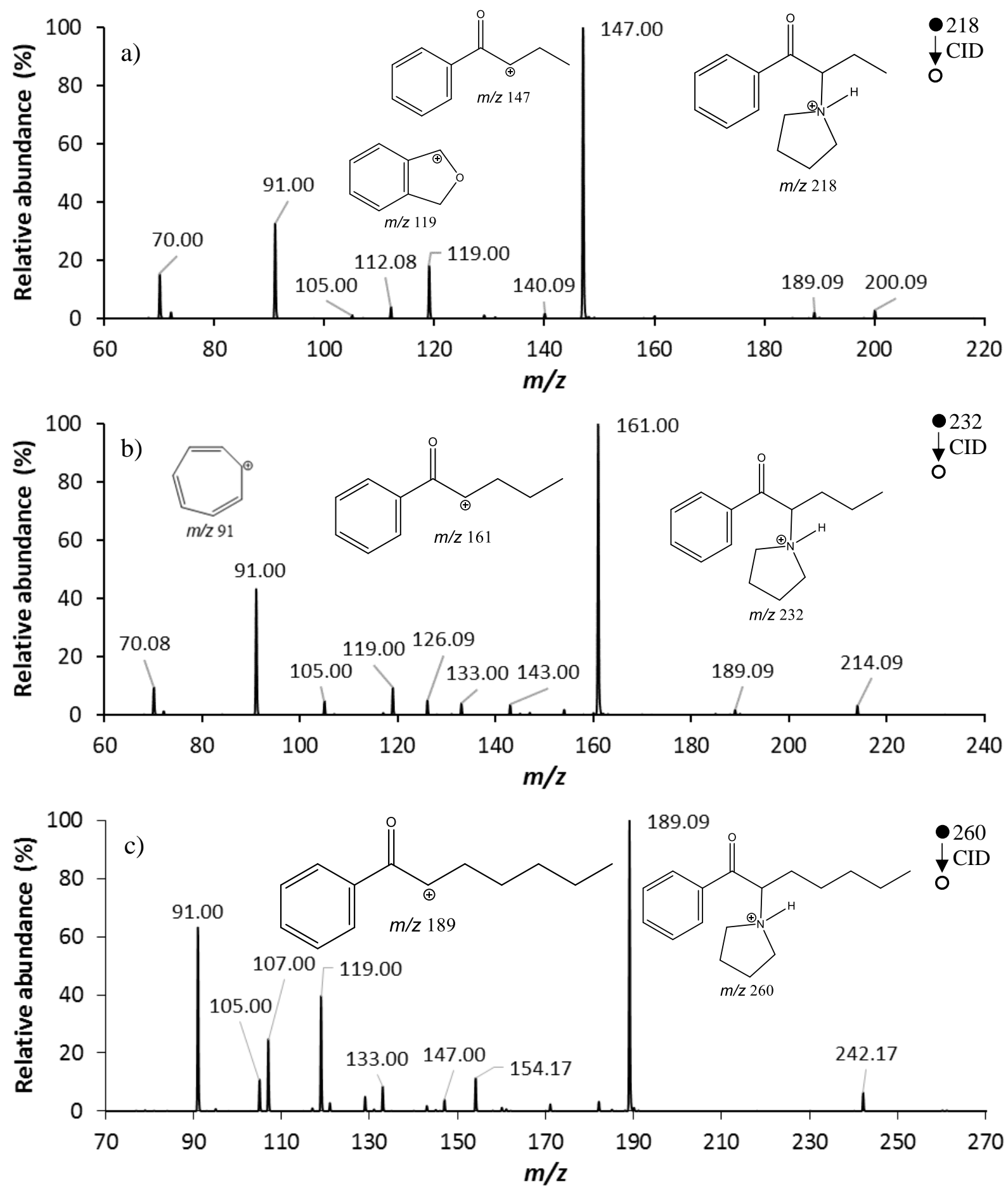

Figure 2.6. Tandem mass spectra of: a) $\alpha$-PBP (35\% NCE), b) $\alpha$-PVP (35\% NCE), and c) PV8 (35\% NCE) demonstrating the effect of the alkyl chain length on the distribution of product ions.

The biggest impact of the identification of the conserved fragmentation pathways described in Figure 2.5 is the application of this information to the identification of emerging synthetic 
cathinones. If a questioned seized drug provides a tandem mass spectrum with an abundant neutral loss of $71 \mathrm{Da}$ from the $[\mathrm{M}+\mathrm{H}]^{+}$precursor, as well as additional peaks consistent with neutral losses of $\mathrm{C}_{6} \mathrm{H}_{6},{ }^{\cdot} \mathrm{C}_{2} \mathrm{H}_{4} \mathrm{C}_{\mathrm{n}} \mathrm{H}_{\mathrm{n} 2+1}, \mathrm{H}_{2} \mathrm{O}$ and $\mathrm{CH}_{2} \mathrm{C}_{\mathrm{n}} \mathrm{H}_{2 \mathrm{n}}$, then the spectrum is consistent will all of the $\alpha-$ pyrrolidinophenone synthetic cathinones in this study. The presence of secondary fragmentation from any of the aforementioned peaks provides additional confidence in the identification of an $\alpha$ pyrrolidinophenone synthetic cathinone.

Additionally, the ability to identify substitution to the core $\alpha$-pyrrolidinophenone synthetic cathinone structure through shifts in the mass axis provides an additional tool for the identification of $\alpha$-pyrrolidinophenone synthetic cathinones. Specifically, the location of the substitution can be identified based on which peaks diverge from the proposed fragmentation pathways in Figure 2.5.

Table 2.1 shows the $[\mathrm{M}+\mathrm{H}]^{+}$protonated precursor and the five most abundant product ions for $13 \alpha$-pyrrolidinophenone synthetic cathinones from this study. The five most abundant fragments are listed in order of decreasing abundance with all comparisons made at a set collision energy within the LIT and Q-TOF data due to the dependence of product ion abundances on the applied collision energy. These 13 standards include 11 non-isotopically labeled and two deuterated $\alpha$ pyrrolidinophenone synthetic cathinones. The product ions in Table 2.1 highlight both the frequency of occurrence for the product ions described in Figure 2.5, and the similarity in fragment ion abundances between the IT and Q-TOF instruments. With the exception of the loss of formaldehyde from methylenedioxy-containing compounds, and the additional methyl group for $\alpha$-PVP-methyl, the only product ion in Table 2.1 that is not described by Figure 2.5 is $m / z, 107$ for PV8 (Figure 2.6c). The proposed elemental formula for the product ion at nominal $\mathrm{m} / \mathrm{z} 107$ is $\mathrm{C}_{7} \mathrm{H}_{7} \mathrm{O}^{+}$, which has a theoretical exact mass of $\mathrm{m} / 2$ 107.0496, about $5 \mathrm{ppm}$ from the measured accurate mass of $m / z 107.0490$ based on Q-TOF data. 
Table 2.1. Protonated precursor and the five most abundant product ions in decreasing order of abundance for 13 of the synthetic cathinones used in this study. Fragment ions are reported for both the LIT and Q-TOF instruments.

\begin{tabular}{|c|c|c|c|}
\hline Compound & {$[\mathbf{M}+\mathbf{H}]^{+}$} & $\begin{array}{c}\text { LIT product ions }(m / z) \\
@ 30 \% \text { NCE }\end{array}$ & $\begin{array}{l}\text { Q-TOF product ions } \\
(m / z) @ 25 \mathrm{eV}\end{array}$ \\
\hline$\alpha-P P P$ & $m / z 204$ & $133,105,70,186,98$ & $\begin{array}{c}105.07,98.09,133.06, \\
70.06,77.03\end{array}$ \\
\hline$\alpha-P B P$ & $m / z 218$ & $147,91,119,70,112$ & $\begin{array}{c}91.05,112.11,105.07 \\
70.06,161.09 \\
\end{array}$ \\
\hline$\alpha-P V P$ & $m / z 232$ & $161,91,70,119,126$ & $\begin{array}{c}91.05,126.12,105.03 \\
70.06,161.09 \\
\end{array}$ \\
\hline$\alpha$-PVP-methyl & $m / z 246$ & $175,105,140,72,228$ & $\begin{array}{c}105.07,72.08,77.04 \\
140.14,98.09 \\
\end{array}$ \\
\hline PV8 & $m / z 260$ & $189,91,119,107,154$ & $\begin{array}{c}91.05,154.16,105.03, \\
70.06,119.04 \\
\end{array}$ \\
\hline 4-MeO- $\alpha-\mathrm{PVP}$ & $m / z 262$ & $191,126,121,163,135$ & $\begin{array}{c}121.06,126.12,135.04, \\
191.10,84.08\end{array}$ \\
\hline 3',4'-trimethylene- $\alpha$-PVP & $m / z 272$ & $201,131,126,145,173$ & $\begin{array}{c}131.08,201.12,126.12, \\
145.06,84.08\end{array}$ \\
\hline 3,4-MDPPP & $m / z 248$ & $177,147,98,149,230$ & $\begin{array}{c}98.09,147.04,149.06 \\
177.05,119.05 \\
\end{array}$ \\
\hline 3,4-MDPBP & $m / z 262$ & $191,161,112,163,149$ & $\begin{array}{c}112.11,161.06,149.02, \\
191.07,163.07\end{array}$ \\
\hline 3,4-MDPV & $m / z 276$ & $205,175,126,135,177$ & $\begin{array}{c}126.12,135.04,175.07, \\
149.02,205.08\end{array}$ \\
\hline 2,3-MDPV & $m / z 276$ & $175,135,205,177,126$ & $\begin{array}{c}135.04,175.07,126.12, \\
149.02,70.06\end{array}$ \\
\hline 3,4-MDPV-d 8 & $m / z 284$ & $205,175,134,135,177$ & $\begin{array}{c}134.17,135.04,149.02, \\
175.07,92.13\end{array}$ \\
\hline$\alpha-P V P-d_{8}$ & $m / z 240$ & $161,91,77,119,134$ & $\begin{array}{c}91.05,134.17,105.03 \\
161.09,77.11\end{array}$ \\
\hline
\end{tabular}

\subsubsection{GC-EI-MS}

Thermal degradation was observed as shouldering or a split peak consistent with previous literature [62-64]; however, the formation of the 2,3-enamine degradation product was always insignificant relative to the abundance of the non-degraded parent compound. The GC-EI-MS data demonstrates that the proposed carbon backbone rearrangements observed for the protonated tandem MS data are insignificant for all compounds analyzed by EI. EI is a hard ionization source, which causes extensive fragmentation with well-established mechanisms, such as radical-directed 
cleavage to form the benzoylium ion at $\mathrm{m} / \mathrm{z}$ 105. The mechanisms of fragmentation of EI-MS are both radical-directed and charge-directed, in contrast to the charge-remote 4-center eliminations that dominate the tandem mass spectra of protonated precursor ions. For example, Figure 2.7a shows the GC-EI-MS spectrum of ${ }^{13} \mathrm{C}-\alpha$-PPP isotopically labeled with a ${ }^{13} \mathrm{C}$ on the $\alpha$-carbon. The major structural fragments are embedded. The spectrum has been truncated due to the lack of high mass ions, such as the molecular ion, which is often missing with EI-MS of synthetic cathinones [15]. The presence of the benzoylium ion at $\mathrm{m} / \mathrm{z} 105$, phenylium ion at $\mathrm{m} / \mathrm{z} 77$ and the dominant 1 ethylidenepyrrolidin-1-ium ion at $m / z 99$ (accounting for ${ }^{13} \mathrm{C}$ ) are all consistent with previous literature on the EI-MS fragmentation of $\alpha$-pyrrolidinophenone synthetic cathinones $[\underline{12}, \underline{13}, \underline{15}$, $\underline{30}, \underline{32}, \underline{65]}$.

Figure 2.7b shows the truncated GC-EI-MS results for the analysis of ${ }^{13} \mathrm{C}-3,4-\mathrm{MDPV}$ labeled with a ${ }^{13} \mathrm{C}$ on the carbonyl carbon. The major structural fragments are embedded. Even with the additional methylenedioxy substitution, the 1-butylidenepyrrolidin-1-ium ion at $\mathrm{m} / \mathrm{z} 126$ is the base peak of this spectrum. However, the methylenedioxy substitution does shift the phenylium and benzoylium ions by 44 Da to the observed peaks at $m / z, 121$ and $m / z, 150$, respectively. The product ion at $m / z 150$ accounts for the $1 \mathrm{Da}$ shift for the ${ }^{13} \mathrm{C}$ present in the substituted benzoylium ion. 

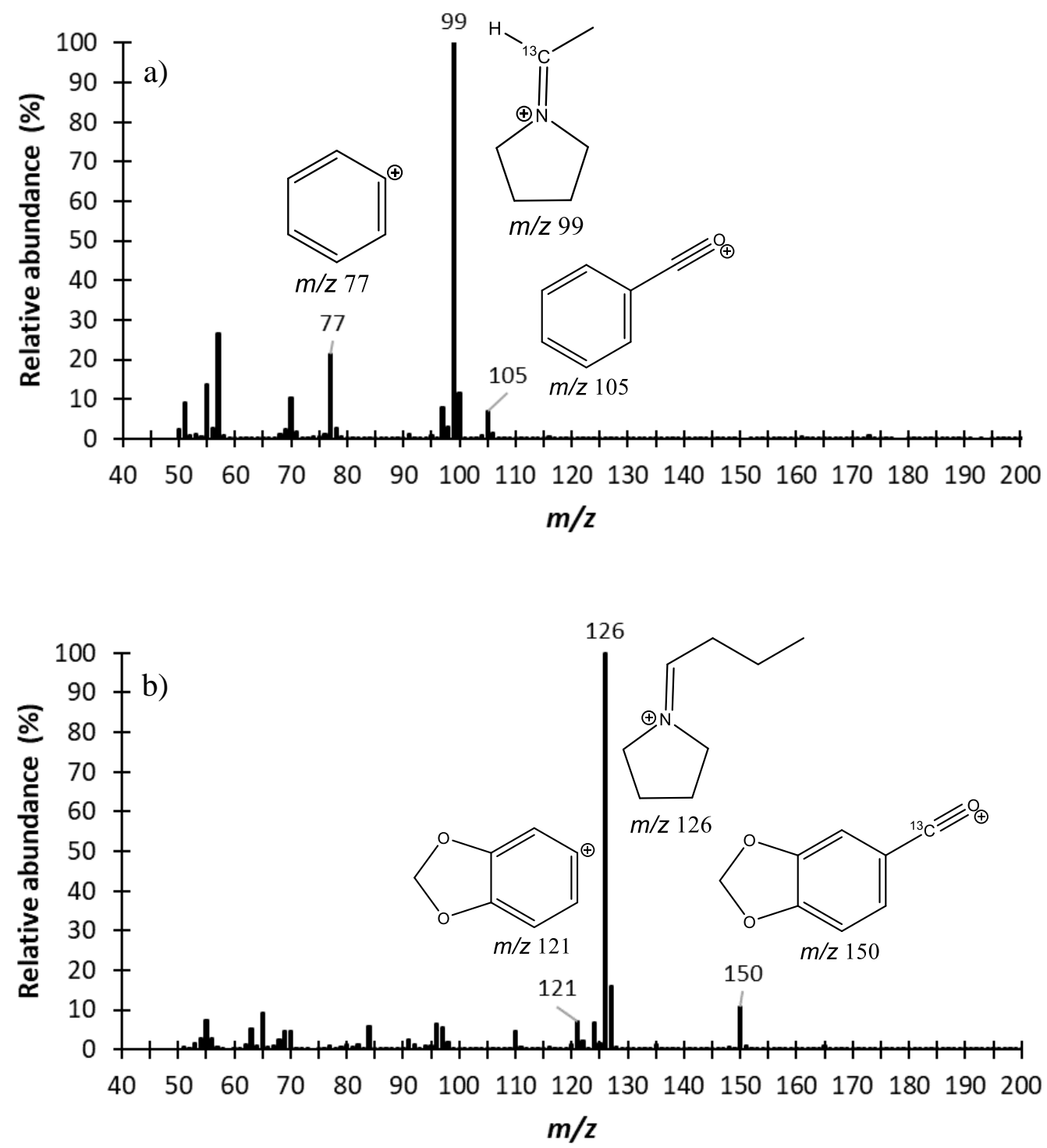

Figure 2.7. Full scan mass spectra of a) ${ }^{13} \mathrm{C}-\alpha$-PPP and b) ${ }^{13} \mathrm{C}-3,4-\mathrm{MDPV}$ collected with GC-EIMS.

The truncated GC-EI-MS results for the analysis of ${ }^{13} \mathrm{C}-\mathrm{PV} 8$ labeled with a ${ }^{13} \mathrm{C}$ on the carbonyl carbon are shown in Figure 2.8 with the major structural fragments embedded. The base peak of this spectrum is the 1-hexylidenepyrrolidin-1-ium ion at $\mathrm{m} / \mathrm{z} 154$, which, as expected, does not contain the ${ }^{13} \mathrm{C}$ from the carbonyl carbon. Other peaks of significant abundance are the benzoylium ion at $m / z 106$ (accounting for ${ }^{13} \mathrm{C}$ ) and the phenylium ion at $\mathrm{m} / \mathrm{z}$ 77. The conserved fragmentation 
pathways through the acylium and iminium ions hold true across the series of substitutions analyzed during this study.

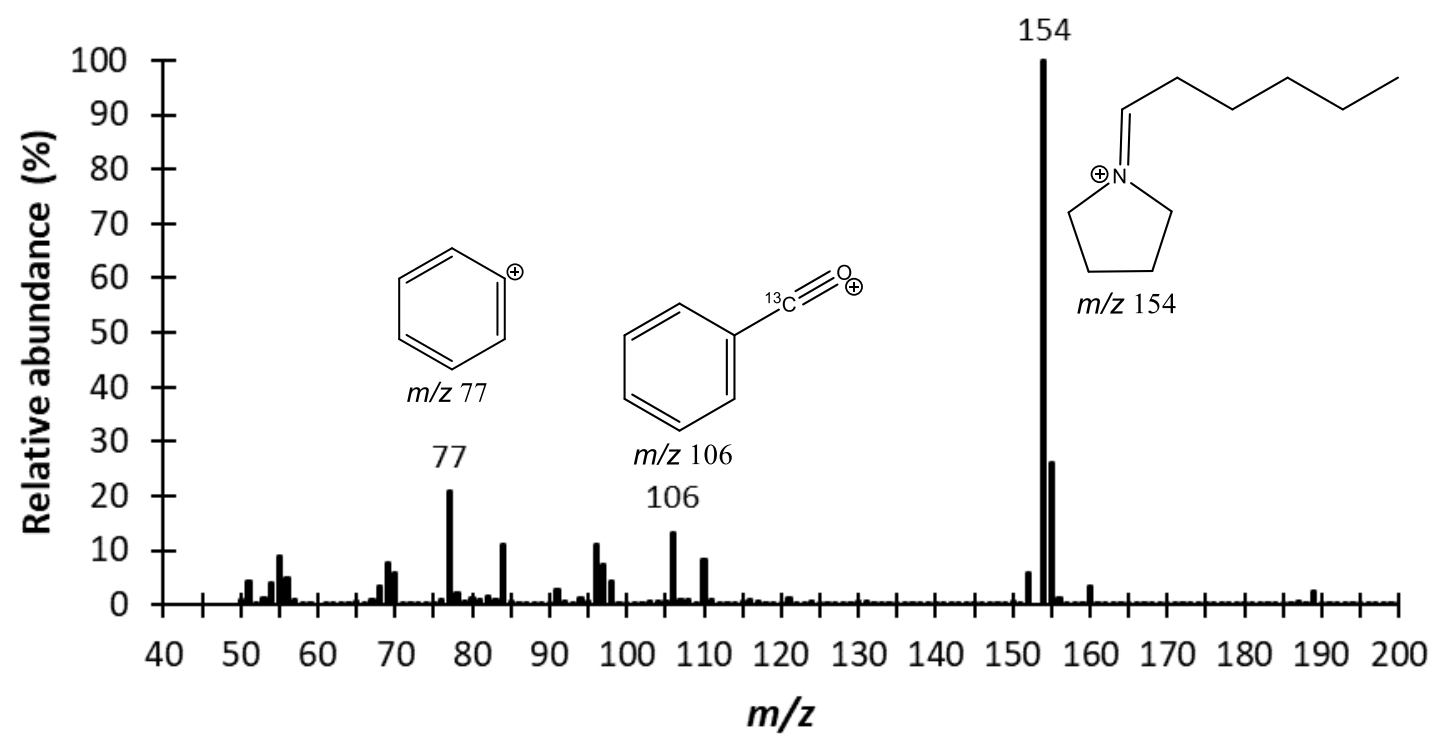

Figure 2.8. Full scan mass spectra of ${ }^{13} \mathrm{C}-\mathrm{PV} 8$ collected with GC-EI-MS.

Figure 2.9 demonstrates the proposed general fragmentation mechanisms for $\alpha$ pyrrolidinophenone synthetic cathinones under EI-MS conditions, where X represents substitution to the aromatic ring moiety and $\mathrm{C}_{\mathrm{n}} \mathrm{H}_{2 \mathrm{n}+1}$ represents varying alkyl chain lengths. The ${ }^{13} \mathrm{C}$, ${ }^{18} \mathrm{O}$, and deuterated labeling are not shown in these proposed general mechanisms, but were used to generate the proposed mechanisms, which are drawn explicitly for $\alpha$-PVP as an example. 


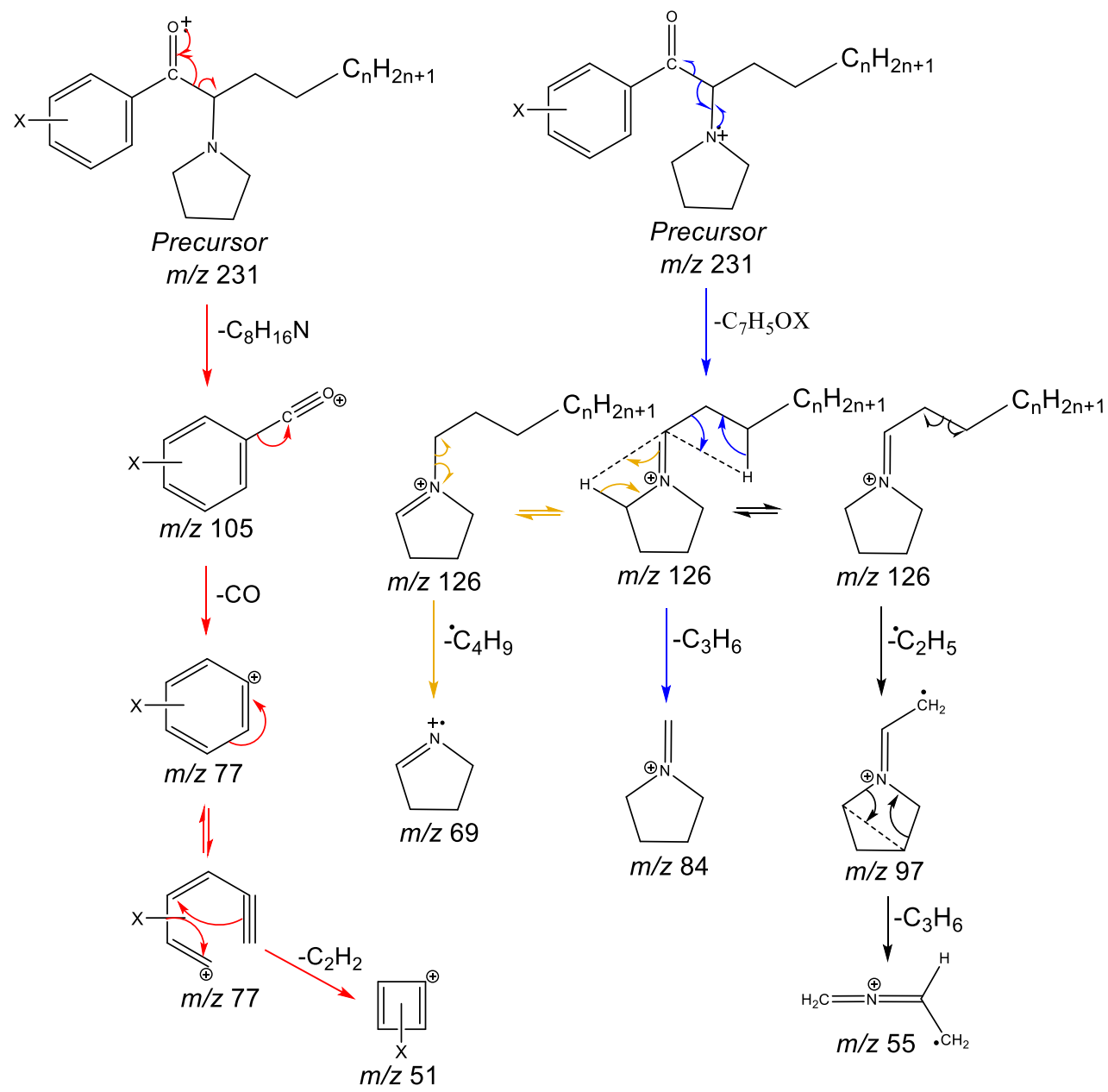

Figure 2.9. Proposed general mechanisms for the fragmentation of $\alpha$-pyrrolidinophenone synthetic cathinones with EI-MS (adapted from references [30, 60, 65]). The model compound is $\alpha$-PVP.

The two most abundant fragmentation pathways of $\alpha$-pyrrolidinophenone synthetic cathinones are acylium and iminium ions, with iminium ions being the most dominant pathway. Acylium ions form through $\alpha$-cleavage initiated by a radical electron on the oxygen, which produces characteristic ions at $\mathrm{m} / \mathrm{z}, 105,77$, and 51 for all the $\alpha$-pyrrolidinophenone synthetic cathinones studied here. However, the presence of iminium ions are far more useful for the differentiation of synthetic cathinone isomers because of the secondary and tertiary fragmentation described by Zuba [15]. The formation of the iminium ion cascade is initiated by a radical electron on the nitrogen 
and $\alpha$-cleavage of the bond between the carbonyl carbon and the $\alpha$-carbon adjacent to the pyrrolidine ring. Secondary iminium ion fragmentation forms characteristic ions, such as those demonstrated for $\alpha$-PVP in Figure 2.9 at $\mathrm{m} / \mathrm{z}$, 97, 84, and 69 through the loss of an ethyl radical $\left({ }^{\circ} \mathrm{C}_{2} \mathrm{H}_{5}\right)$, propylene, and a butyl radical $\left({ }^{\cdot} \mathrm{C}_{4} \mathrm{H}_{9}\right)$, respectively. The secondary iminium ion at $\mathrm{m} / z$ 97 further fragments into the tertiary iminium ion at $\mathrm{m} / \mathrm{z} 55$ through the loss of cyclopropane.

\subsection{Conclusions}

The combination of isotope-labeling, $\mathrm{MS}^{\mathrm{n}}$, and HRMS was used to study the fragmentation behavior of $\alpha$-pyrrolidinophenone synthetic cathinones to gain a deeper understanding about the characteristic fragmentation pathways of this class of synthetic cathinone analogs. Three instruments that are common in toxicology laboratories and crime laboratories were used to develop characteristic fragmentation pathways to assist practitioners with the identification of $\alpha$ pyrrolidinophenone synthetic cathinones. Through the analysis of 22 -pyrrolidinophenone synthetic cathinones, ESI and DART ionization sources on the same Q-TOF mass spectrometer produced even-electron protonated molecular ions and almost indistinguishable tandem mass spectra. The fragmentation pathways are highly conserved between the LIT and Q-TOF mass spectrometers, although the multi-collisional environment of the ion trap occasionally tends to limit the extent of consecutive fragmentations relative to the Q-TOF instrument [35]. The Q-TOF therefore favored the abundant production of lower mass ions relative to the LIT instrument.

The identification of conserved tandem mass spectrometry fragmentation pathways through the loss of $\mathrm{CH}_{2} \mathrm{C}_{\mathrm{n}} \mathrm{H}_{2} \mathrm{n}, \mathrm{H}_{2} \mathrm{O},{ }^{\cdot} \mathrm{C}_{2} \mathrm{H}_{4} \mathrm{C}_{\mathrm{n}} \mathrm{H}_{\mathrm{n} 2+1}, \mathrm{C}_{6} \mathrm{H}_{6}$, and $\mathrm{NC}_{4} \mathrm{H}_{9}$ from protonated molecular ions provides a series of diagnostic ions that can be used for the tandem mass spectrometry identification of $\alpha$-pyrrolidinophenone synthetic cathinones. Particularly, the dominant pathways through the loss of pyrrolidine and the formation of iminium ions of varying side chain lengths 
provides a technique for structural elucidation through mass axis shifts due to additional substitutions to the core synthetic cathinone structure. The presence of diagnostic ions such as the tropylium ion at $m / z, 91$, substituted iminium ions (i.e. $m / z, 126$ vs $m / z, 112$ ), and the phenylethyl derivative at $m / z 105$ provide key information about the length of the alkyl chain and substitutions to the aromatic ring moiety.

When GC-EI-MS is used to analyze $\alpha$-pyrrolidinophenone synthetic cathinones, the fragmentation pathways are dominated by the formation of iminium and acylium ions as previously reported in literature $[\underline{12}, \underline{13}, \underline{15}, \underline{30}, \underline{32}, \underline{65]}$. This is expected due to the large energy deposition through $70 \mathrm{eV}$ electron fragmentation resulting in direct $\alpha$-cleavage fragmentation rather than the low-energy rearrangements observed with collisional activation. However, the observed fragmentation mechanisms remain unaffected by substitutions to the core synthetic cathinone structure, which provides a rapid method for the identification of novel $\alpha$ pyrrolidinophenone synthetic cathinones through known fragmentation pathway shifts along the mass axis. For example, the product ions observed at $\mathrm{m} / \mathrm{z} 149$ and $\mathrm{m} / \mathrm{z} 121$ for the GC-EI-MS fragmentation of 3,4-MDPV are $44 \mathrm{Da}$ larger than the product ions at $\mathrm{m} / \mathrm{z} 105$ and $\mathrm{m} / \mathrm{z} 77$ for nonmethylenedioxy substituted synthetic cathinones. This study highlights the differences between high energy radical-driven fragmentation in EI and lower energy collisional activation of protonated precursor ions. However, knowledge about the systematic tendencies of both techniques can be used to help support the identification of emerging synthetic drugs. 


\section{Chapter 3: Fragmentation pathways of odd- and even-electron $N$-alkylated synthetic}

cathinones

Reproduced in part with permission from J.T. Davidson, Z.J. Sasiene, G.P. Jackson, International Journal of Mass Spectrometry, DOI: 10.1016/j.ijms.2020.116354.

\subsection{Introduction}

$\mathrm{N}$-alkylated synthetic cathinones are analogs of the natural product cathinone, which is derived from the leaves of the Catha edulis plant, commonly referred to as khat. Khat is a native plant to the Horn of Africa and the Southwest Arabian Peninsula that, when chewed, produces stimulantlike effects $[1,66]$. The stimulant properties of cathinones are typically stronger than over-thecounter stimulants like caffeine and nicotine, and similar to the effects of amphetamines, which are structurally similar to cathinones [7]. $\mathrm{N}$-alkylated synthetic cathinones are characterized by alkyl side chains on the amine moiety of the generic benzoylethanamine structure. Additional analogs within this class include those with aliphatic substitutions to the alkyl chain and with substitutions to the benzene ring. The $N$-alkylated class of compounds was the first class of synthetic cathinone derivatives that became available on the drug market [1].

In the 1930s, methcathinone was marketed under the name ephedrone as an antidepressant in the USSR. Methcathinone was also developed as a central nervous system stimulant by the Parke Davis pharmaceutical company in the United States [7]. In the 1950s, the $N$-alkylated synthetic cathinone diethylpropion was marketed under the name amfepramone as an appetite suppressant [67]. Whereas $N$-alkylated synthetic cathinones were originally developed for therapeutic purposes, the 1970s brought reports of methcathinone abuse in the Soviet Union. By the 1990s, the United States also documented methcathinone abuse [63]. Due to the low cost and psychostimulant nature of synthetic cathinones, substances such as 3,4- 
methylenedioxymethcathinone, better known as methylone were sold in head shops and the internet by the early 2000s [68]. The main reason for abuse of synthetic cathinones was for their recreational use at dance clubs and parties [1].

Currently, synthetic cathinones are marketed as "not for human consumption" or "bath salts" to avoid legislative restrictions imposed to decrease the sale and distribution of these compounds $[2,3]$. The reported symptoms of synthetic cathinone abuse include euphoria, hallucinations, psychosis, paranoia, agitation, violent behavior, tachycardia, acidosis, seizures, and even death $[\underline{53}, \underline{54]}$. The first $N$-alkylated synthetic cathinone to be classified as a schedule I substance by the Drug Enforcement Administration (DEA) was methcathinone in 1993 [69], but the rapid modifications to the generic synthetic cathinone structure make it difficult to regulate. The clandestine synthesis of analogs with only minor modifications to the generic structure provides a way around the imposed regulations while also introducing potentially more harmful substances onto the illicit drug market $[\underline{1}, \underline{70}]$.

This project describes the fragmentation pathways of $N$-alkylated synthetic cathinones using three common analytical techniques available in crime laboratories and national laboratories, including gas chromatography-electron ionization-mass spectrometry (GC-EI-MS), electrospray ionization and tandem mass spectrometry (ESI-MS/MS), and direct analysis in real time (DART) with high-resolution mass spectrometry (DART-HRMS). The DART source was mounted on a quadrupole time-of-flight (Q-TOF) mass spectrometer, which also enables high-resolution MS/MS acquisition. These setups are representative of the instrumentation commonly employed in seized drug laboratories, toxicological laboratories, and national laboratories, respectively. The comparison of the fragmentation mechanisms of odd-electron ions (EI) and even-electron ions (ESI) provides a more comprehensive understanding of the differences in fragmentation behavior. 
The fragmentation behavior of $N$-alkylated synthetic cathinones has been reported throughout literature; however, the underlying fragmentation mechanisms that lead to the observed fragment ions are rarely discussed or understood $[13, \underline{32}, \underline{37}, \underline{71}]$. Examples of the confusion about the formation of the tropylium ion from protonated synthetic cathinones have been highlighted previously by our group [61]. Even when mechanisms have been proposed, the lack of isotopic labeling and multi-stage mass spectrometry limits the certainty associated the proposed mechanisms [28]. Specific examples of reported $N$-alkylated fragmentation behavior without mechanistic understanding including the works of Jankovics et al. [17], Martinez-Clemente et al. [72], and Fornal [16]. These articles provide useful fragmentation pathways but highlight that the mechanistic explanation for the fragmentation behavior of $\mathrm{N}$-alkylated synthetic cathinones remain unclear. By gaining a better understanding of the fragmentation mechanisms, analysist would be better positioned to both defend the data of known scheduled drugs and to perform structural characterization to identify novel psychoactive substances (NPS) entering the market [13].

The goal of this project is to better understand the fragmentation pathways of $N$-alkylated synthetic cathinones. The developed fragmentation pathways and mechanistic explanations will help advance the current understanding of the behavior of $\mathrm{N}$-alkylated synthetic cathinones under different ionization and fragmentation conditions. Specifically, the comparison between the fragmentation behavior of odd-electron (via EI) and even-electron (via ESI and DART) ions of different $N$-alkylated synthetic cathinones provides a more comprehensive understanding of these compounds. This project combines isotopic labeling, $\mathrm{MS}^{\mathrm{n}}$, and accurate mass measurements with HRMS to confirm the mechanisms of odd-electron and even-electron fragmentation of $N$-alkylated synthetic cathinones. 


\subsection{Methods}

\subsubsection{Sample Preparation}

The ten standards purchased through Cayman Chemical (Ann Arbor, MI, USA) were methcathinone, ethcathinone, pentedrone, buphedrone, $\alpha$-propylaminopentiophenone, $N$ ethylbuphedrone, 3,4-dimethyl- $\alpha$-ethylaminovalerophenone, methylone, butylone, and pentylone. The nine standards purchased through Cerilliant (Round Rock, TX, USA) were methcathinone- $\mathrm{d}_{3}$ ( $N$-alkyl deuterated), diethylpropion, diethylpropion- $\mathrm{d}_{10}\left(\mathrm{~N}\right.$-alkyl deuterated), ${ }^{13} \mathrm{C}$-benzedrone $\left({ }^{13} \mathrm{C}\right.$ on carbonyl carbon), ${ }^{13} \mathrm{C}$-ethylone $\left({ }^{13} \mathrm{C}\right.$ on carbonyl carbon), ${ }^{13} \mathrm{C}$-butylone $\left({ }^{13} \mathrm{C}\right.$ on carbonyl carbon), pentylone- $\mathrm{d}_{3}$ ( $N$-alkyl deuterated), dibutylone- $\mathrm{d}_{3}$ (alkyl deuterated), and eutylone- $\mathrm{d}_{5}(N-$ alkyl deuterated). The non-deuterated samples were prepared in a solution of 49\% HPLC grade methanol, $49 \%$ distilled water, and 2\% acetic acid. The HPLC grade methanol was supplied by Fisher Scientific (Palo Alto, CA) and the acetic acid was supplied by Acros Organics (Palo Alto, CA). The deuterated samples were left in the original methanol solvent. All samples were prepared to a final concentration of approximately $100 \mathrm{ppm}$.

\subsubsection{Instrumentation}

\subsubsection{Linear Ion Trap}

A Thermo Scientific Velos Pro Linear Ion Trap (LIT) mass spectrometer was operated with heated-electrospray ionization (HESI) operated at $50{ }^{\circ} \mathrm{C}$. The spray voltage was $4,000 \mathrm{~V}$ with the nitrogen sheath gas flow set to 8 arbitrary units and the nitrogen auxiliary flow set to 5 arbitrary units. The mass spectrometer capillary temperature was set to $275{ }^{\circ} \mathrm{C}$. The scan range and normalized collision energy (NCE) were specific for each compound and are labeled with each mass spectrum. The bath gas was ultra-pure helium from Matheson TRIGAS (Fairmont, WV, USA). 


\subsubsection{Quadrupole Time-of-Flight}

An Agilent Technologies 6538 UHD Accurate-Mass Quadrupole Time-of-Flight (Q-TOF) mass spectrometer was operated with both a dual ESI source and a direct analysis in real time (DART) source. The DART-100 source was mounted to the Q-TOF with a Vapur® interface (IonSense, Saugus, MA, USA). The DART ion source was operated with helium gas at $300{ }^{\circ} \mathrm{C}$, with a flow rate of $3.0 \mathrm{~L} / \mathrm{min}$, a grid voltage of $400 \mathrm{~V}$ and a needle voltage of 3,500 V. The ESI source was operated with a spray voltage of $3,500 \mathrm{~V}$ with a $300{ }^{\circ} \mathrm{C}$ nitrogen drying gas flow rate of $5 \mathrm{~L} / \mathrm{min}$ and a nebulizer flow of $30 \mathrm{psig}$. The MS fragmentor and skimmer voltages, the scan range and collision energy were specific for each compound and are labeled with each mass spectrum. An isolation width of 1.3 Da was used for all samples. The ultra-high purity nitrogen used for the collision gas and the ultra-high purity helium used for the DART gas were purchased through Matheson TRIGAS (Fairmont, WV, USA).

The DART samples were prepared through the deposition of $5 \mu \mathrm{L}$ of drug standard onto the closed end of a 1.5 x 1.8 x $90 \mathrm{~mm}$ Pyrex® glass capillary purchased through Corning Life Sciences (Corning, New York, USA). Once the samples were dry, the DART analysis consisted of approximately $30 \mathrm{~s}$ of background collection, about $5 \mathrm{~s}$ of sample introduction, and then the

analysis of a blank capillary to account for capillary specific background. The total length of analysis was less than $90 \mathrm{~s}$ per sample.

\subsubsection{Single Quadrupole GC-EI-MS}

An Agilent Technologies 7890 GC-5977 MS with a HP-5 ((5\% phenyl)-methylpolysiloxane) $30 \mathrm{~m} \times 250 \mu \mathrm{m} \times 0.25 \mu \mathrm{m}$ column manufactured by Agilent J\&W Columns was used for these analyses. The GC-EI-MS parameters were as follows: injection volume was $1 \mu \mathrm{L}$; injection temperature was $250^{\circ} \mathrm{C}$; split ratio was $20: 1$. The initial oven temperature was $80^{\circ} \mathrm{C}(1 \mathrm{~min}$ hold), 
which was ramped to $280{ }^{\circ} \mathrm{C}$ at $15{ }^{\circ} \mathrm{C} / \mathrm{min}$, then held for $2 \mathrm{~min}$. The carrier gas (helium) flow rate was set to $1 \mathrm{~mL} / \mathrm{min}$ and the transfer line temperature was set to $280{ }^{\circ} \mathrm{C}$. The ultra-high purity helium gas was purchased through Matheson TRIGAS (Fairmont, WV, USA). The mass spectrometer was scanned from $\mathrm{m} / \mathrm{z} 50-500$ after a solvent delay of $2 \mathrm{~min}$. The scan rate was 1,500 $\mathrm{Da} / \mathrm{sec}$. The source and quadrupole temperatures were $250{ }^{\circ} \mathrm{C}$ and $200{ }^{\circ} \mathrm{C}$, respectively.

\subsubsection{Data Analysis}

Xcalibur 2.0.0.48 software was used for the data analysis on the Velos Pro instrument. MassHunter Qualitative Analysis B.05.00 was used for the Agilent Q-TOF data analysis, and ChemStation version C.01.01 was used for the Agilent GC-EI-MS data analysis. Microsoft Excel version 14 (Microsoft, Redmond, WA, USA) and ChemDraw 16.0 (PerkinElmer, Waltham, MA, USA) were used for mass spectral plots and mass spectral fragmentation mechanisms.

\subsubsection{Mass Spectral Interpretation and Mechanisms}

The mass spectral fragmentation mechanisms proposed are based on the combination of isotopic labeling, $\mathrm{MS}^{\mathrm{n}}$ and accurate mass measurements with HRMS. The mechanisms follow the expected lowest energy pathways [35], which often involve charge-remote 4-center eliminations consistent with previous literature $[\underline{73}, \underline{74}]$. The isotopic labeling consisted of per-deuteration on both the alkyl chains and the $\mathrm{N}$-alkyl chains and ${ }^{13} \mathrm{C}$ on the carbonyl carbons. These isotopic labels provide useful information through the loss of isotopically shifted neutrals. Even though it is not always possible to know the exact location of hydrogen or deuterium atoms in certain rearrangements, the use of $\mathrm{MS}^{\mathrm{n}}$ and HRMS helps identify the structure of intermediates along the proposed pathways and the elemental composition, respectively. The $N$-alkylated class of synthetic cathinones has been shown to form odd-electron product ions from even-electron (e.g. protonated) precursor ions [36, 37], which is consistent with the present work. 


\subsection{Results/Discussion}

\subsubsection{HESI-Velos Pro MS}

Figure 3.1 shows the $M S^{n}$ fragmentation of pentedrone with the major structural fragments embedded. Isolation and fragmentation of the precursor ion $[\mathrm{M}+\mathrm{H}]^{+}$at $\mathrm{m} / z, 192$ results in the production of two dominant product ions at $\mathrm{m} / \mathrm{z} 174$ and $\mathrm{m} / \mathrm{z} 161$ (Figure 3.1a). The product ion at $m / z, 174$ forms through the loss of $\mathrm{H}_{2} \mathrm{O}$, whereas the product ion at $\mathrm{m} / z 161$ forms through the loss of the $N$-alkylated moiety. Figure 3.1b shows the $\mathrm{MS}^{3}$ spectrum from the isolation and fragmentation of the primary product ion at $\mathrm{m} / \mathrm{z}, 174$. The major secondary fragments of $\mathrm{m} / \mathrm{z} 174$ appear at $m / z 159,145,132$, and 131 . These losses are consistent with the loss of a methyl radical $\left({ }^{\circ} \mathrm{CH}_{3}\right)$, ethyl radical $\left({ }^{\circ} \mathrm{C}_{2} \mathrm{H}_{5}\right)$, propylene $\left(\mathrm{C}_{3} \mathrm{H}_{6}\right)$, and propyl radical $\left({ }^{\circ} \mathrm{C}_{3} \mathrm{H}_{7}\right)$, respectively, with the propylene pathway being the dominant pathway. Figure 3.1c shows the resulting spectrum from the isolation and fragmentation of the primary product ion at $\mathrm{m} / \mathrm{z} 161$. The secondary product ions observed from the primary fragment at $\mathrm{m} / \mathrm{z} 161$ are consistent with the valerophenone ion fragmentation pathways previously demonstrated by our group [75] including the dominant tropylium ion. As described previously for $\alpha$-pyrrolidinophenone synthetic cathinones, the tropylium fragment almost certainly contains the $\alpha$-carbon and not the carbonyl carbon and forms via a phthalane-like intermediates at $\mathrm{m} / \mathrm{z} 119$ and $\mathrm{m} / \mathrm{z} 133$ [61]. 

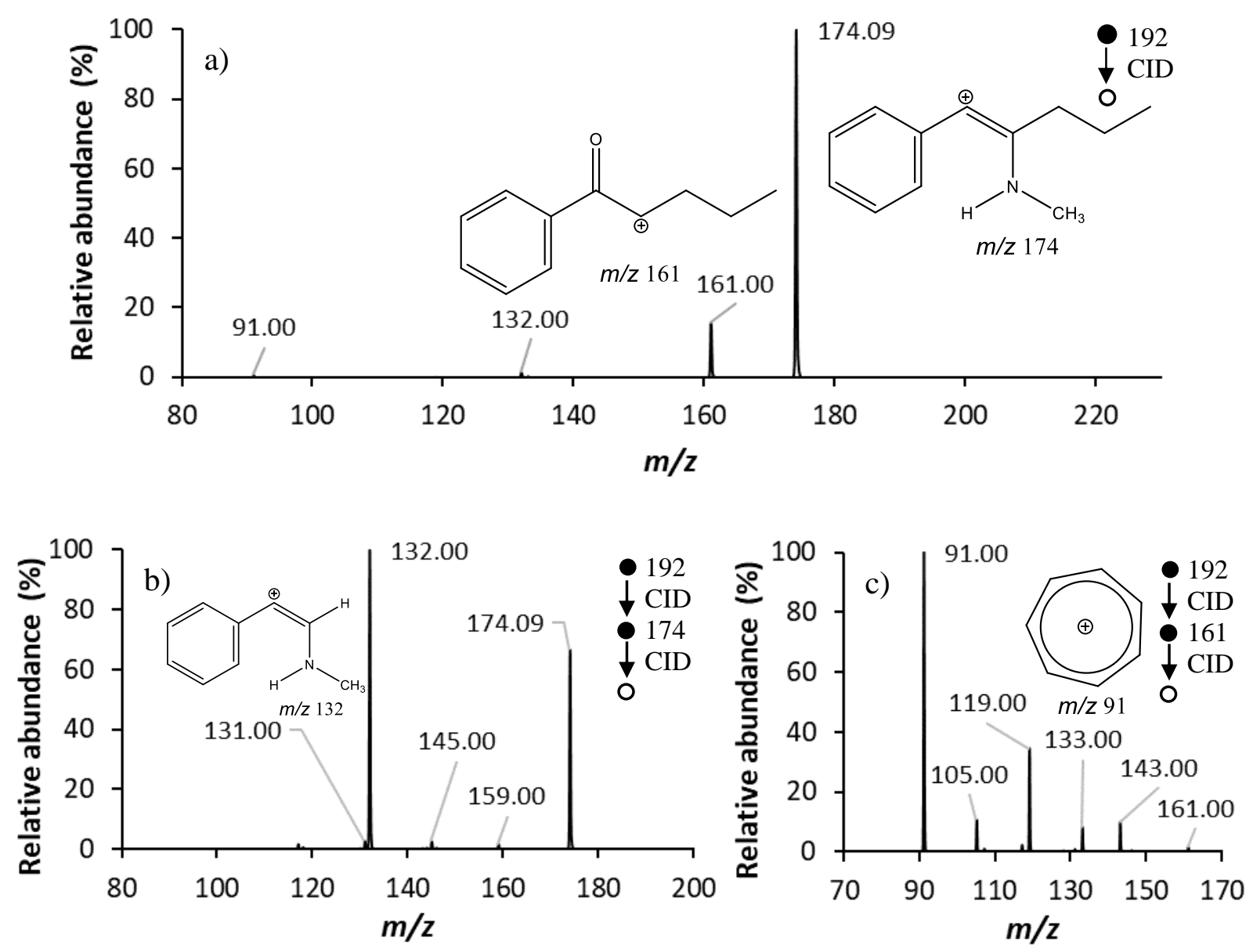

Figure 3.1. Product ion mass spectra of pentedrone on the LIT: a) product ion spectrum of the $[\mathrm{M}+\mathrm{H}]^{+}$molecular ion $(30 \% \mathrm{NCE})$; b) product ion spectrum of the product ion at $\mathrm{m} / \mathrm{z}, 174(30 \%$ NCE) showing the formation of secondary product ions at $m / z 159,145,132$, and 131; c) product ion spectrum of the primary product ion at $\mathrm{m} / \mathrm{z} 161(30 \% \mathrm{NCE})$ showing the characteristic valerophenone ion fragmentation.

Figure 3.2 contains the $\mathrm{MS}^{\mathrm{n}}$ fragmentation of pentylone with the major structural fragments embedded. Pentylone is the methylenedioxy substituted equivalent of pentedrone and has a protonated precursor ion at $m / z 236$, which is 44 Da larger than pentedrone. The product ion spectrum of pentylone obtained on the LIT shows product ions at $m / z, 218,205,188,175$, and 86 . The primary product ions at $\mathrm{m} / \mathrm{z} 218$ and $\mathrm{m} / \mathrm{z}, 205$ are consistent with the methylenedioxy derivatives of the primary product ions observed for pentedrone at $m / z \quad 174$ and $\mathrm{m} / \mathrm{z}, 161$, respectively. Also, the methylenedioxy substitution provides two additional fragmentation 
pathways not available or prominent for pentedrone; they are the loss of formaldehyde $\left(\mathrm{CH}_{2} \mathrm{O}\right)$ from the substituted valerophenone-like intermediate and the formation of an iminium ion, as seen by the product ions at $m / z 175$ and $m / z 86$, respectively.

Figure 3.2b shows the $\mathrm{MS}^{3}$ spectrum following isolation and fragmentation of the primary product ion at $m / z 218$, which results in the formation of secondary product ions at $\mathrm{m} / \mathrm{z} 188,176$, and 175. The secondary product ions are consistent with the loss of formaldehyde $\left(\mathrm{CH}_{2} \mathrm{O}\right)$, propylene $\left(\mathrm{C}_{3} \mathrm{H}_{6}\right)$, and a propyl radical $\left({ }^{\circ} \mathrm{C}_{3} \mathrm{H}_{7}\right)$ from the vinyl intermediate at $\mathrm{m} / z 218$, with the loss of formaldehyde from the methylenedioxy group being the dominant pathway. Figure 3.2c shows the resulting mass spectrum from the isolation and fragmentation of the primary product ion at $m / z 205$, which again is dominated by the loss of formaldehyde at $m / z, 175$ and the formation of the methylenedioxy-substituted tropylium ion at $\mathrm{m} / \mathrm{z} 135$ [61]. 

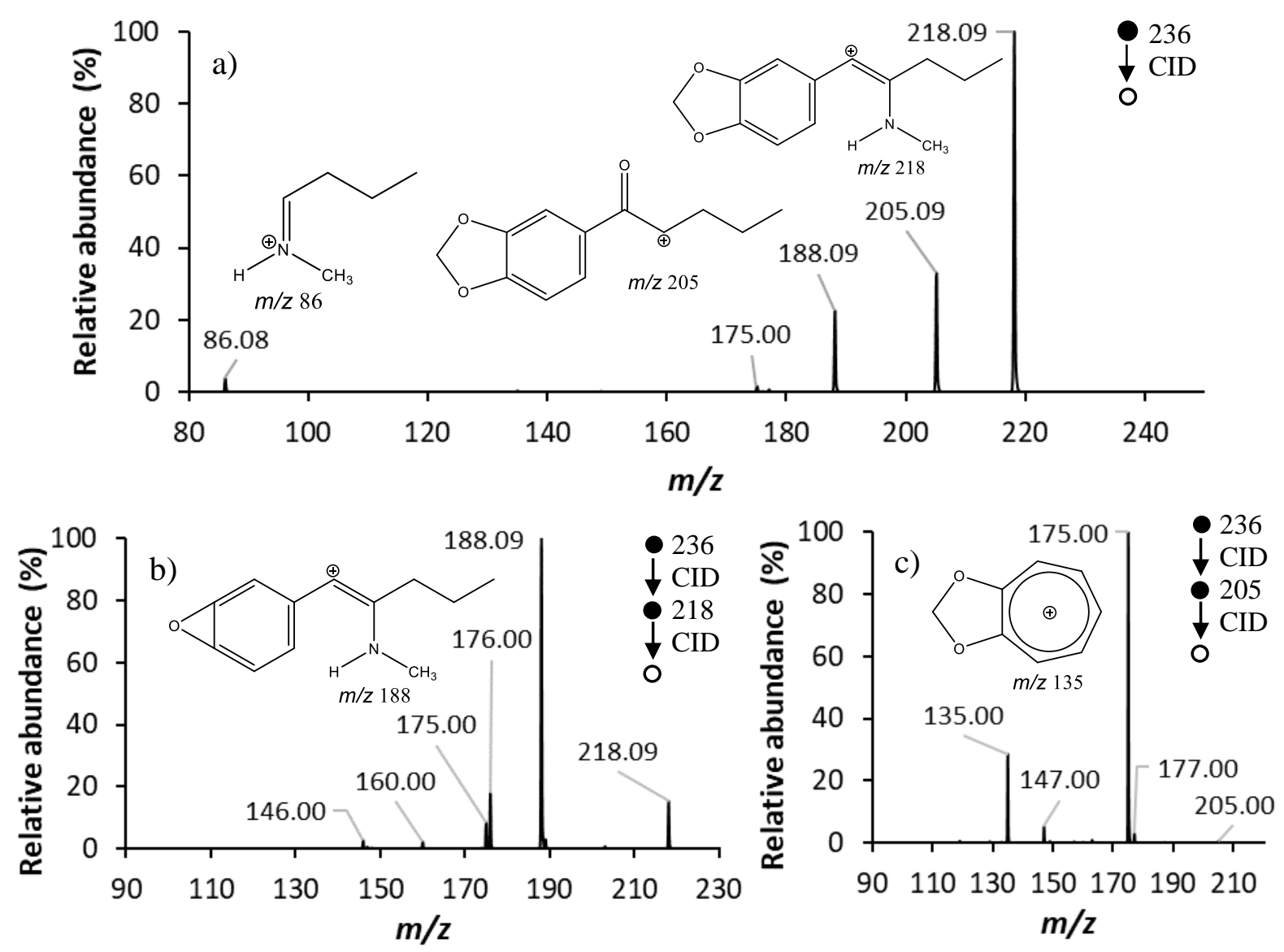

Figure 3.2. Product ion mass spectra of pentylone: a) product ion spectrum of the $[\mathrm{M}+\mathrm{H}]^{+}$ molecular ion (30\% NCE); b) product ion spectrum of the product ion at $\mathrm{m} / z 218$ (30\% NCE) showing the formation of secondary product ions at $\mathrm{m} / \mathrm{z}, 176,175,160$, and 146; c) product ion spectrum of the primary product ion at $\mathrm{m} / \mathrm{z} 205$ (30\% NCE) showing the characteristic methylenedioxy valerophenone ion fragmentation.

Figure 3.3 shows the $\mathrm{MS}^{\mathrm{n}}$ fragmentation of dibutylone- $\mathrm{d}_{3}$, which is deuterated on the end of the alkyl chain. The major structural fragments are embedded. Isolation and fragmentation of the protonated precursor ion $[\mathrm{M}+\mathrm{H}]^{+}$at $\mathrm{m} / z, 239$ results in the formation of major product ions at $\mathrm{m} / \mathrm{z}$ 194,149 , and 89 . The primary product ion at $m / z 194$ forms through the loss of $N$-alkylated moiety whereas the product ions at $\mathrm{m} / \mathrm{z} 149$ and $\mathrm{m} / \mathrm{z} 89$ are the methylenedioxy substituted benzoylium ion and a deuterated iminium ion, respectively. The significance of the tandem mass spectrum in Figure 3.3 is that, in contrast to pentedrone in Figure 3.1, the loss of water from dibutylone- $\mathrm{d}_{3}$ is negligible. We presume that the $N, N$-demethylation hinders hydrogen transfer to the carbonyl 
oxygen and makes a more labile leaving group, both of which make the loss of the $N$-alkyl moiety the base peak for dibutylone- $\mathrm{d}_{3}$. Of the 15 unique $N$-alkylated structures studied, the only other compound to lose the $N$-alkylated moiety in preference to water was diethylpropion, which is also a tertiary amine.

Isolation and fragmentation of the primary product ion of dibutylone- $\mathrm{d}_{3}$ at $\mathrm{m} / \mathrm{z} 194$ results in the formation of secondary product ions at $m / z 166$ and $\mathrm{m} / \mathrm{z}$ 164, which form through the neutral losses of $\mathrm{CO}$ and formaldehyde $\left(\mathrm{CH}_{2} \mathrm{O}\right)$, respectively (Figure 3.3b). Based on $\mathrm{MS}^{\mathrm{n}}$ analysis (not shown), the tertiary product ion at $\mathrm{m} / \mathrm{z} 136$ forms through the loss of CO from the intermediate product ion at $m / z 164$ and the loss of formaldehyde from the intermediate at $m / z$ 166. Figure 3.3c shows the $\mathrm{MS}^{3}$ mass spectrum that results from the isolation and fragmentation of the primary product ion at $\mathrm{m} / \mathrm{z}$ 89. Interestingly, Figure 3.3c shows the presence of product ions at both $\mathrm{m} / \mathrm{z}$ 74 and $m / z 71$ for the losses of ${ }^{\circ} \mathrm{CH}_{3}$ or ${ }^{\circ} \mathrm{CD}_{3}$ radicals, respectively, with a clear preference to lose the terminal ${ }^{\circ} \mathrm{CD}_{3}$ group most distal to the nitrogen. 

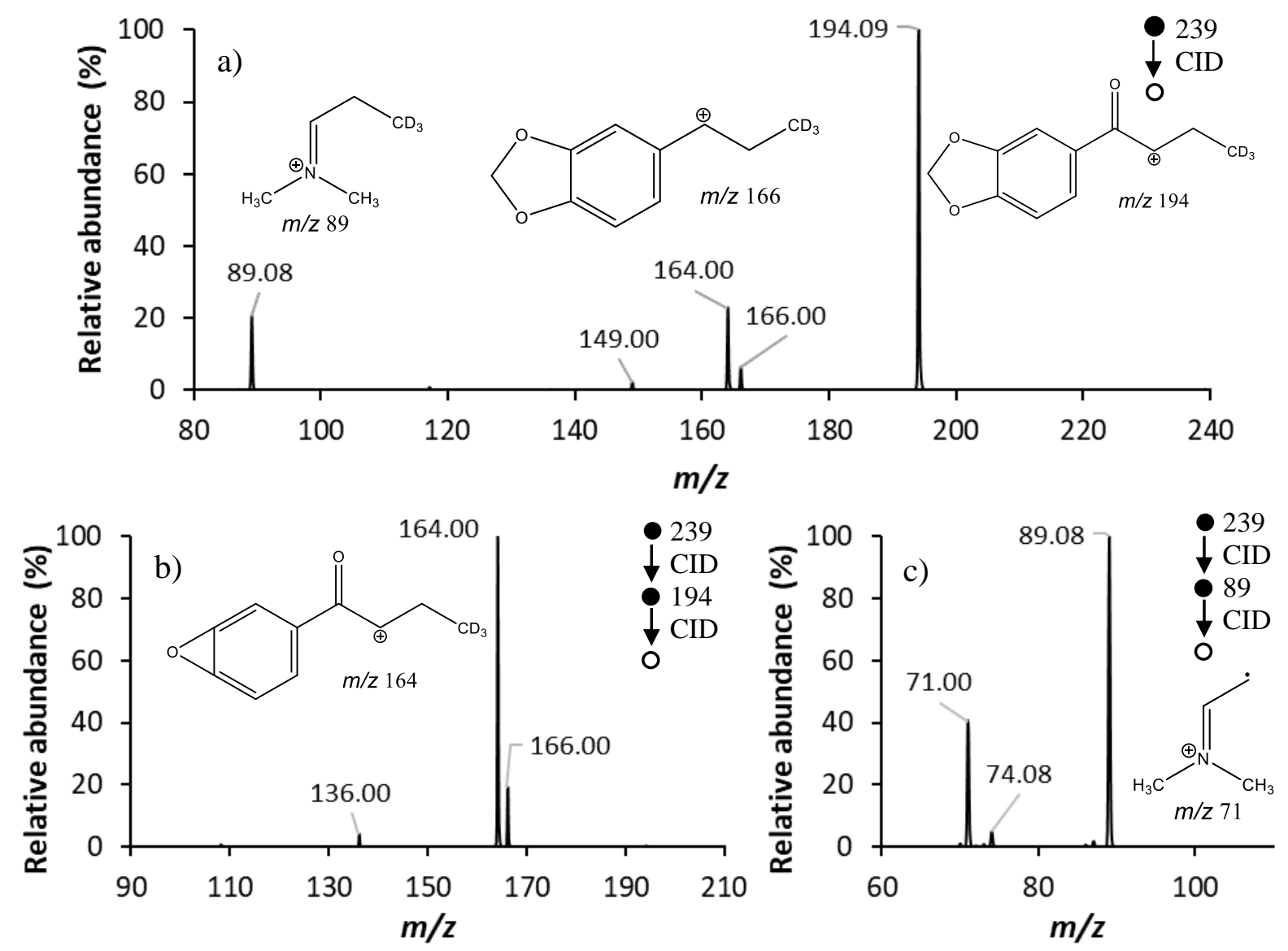

Figure 3.3. Product ion mass spectra of dibutylone- $d_{3}$ on the LIT: a) product ion spectrum of the $[\mathrm{M}+\mathrm{H}]^{+}$molecular ion $(30 \% \mathrm{NCE})$; b) product ion spectrum of the primary product ion at $m / z 194(30 \%$ NCE) showing the formation of product ions at $m / z 166,164$, and 136; c) product ion spectrum of the primary product ion at $m / z 89$ (30\% NCE) showing the loss of both a methyl radical and a deuterated methyl radical.

\subsubsection{DART/ESI-Q-TOF}

Figure 3.4 compares ESI and DART MS/MS spectra of methcathinone- $\mathrm{d}_{3}$ with the major structural fragments embedded. The fragment ion $\mathrm{m} / \mathrm{z}$ values and abundances for DART-MS/MS and ESI-MS/MS are generally similar throughout the spectra. One notable difference between the DART- and ESI-generated mass spectra is the presence of $[\mathrm{M}+\mathrm{H}]^{+}$precursor ion at $\mathrm{m} / \mathrm{z} 167.1322$ $\left(\mathrm{C}_{10} \mathrm{H}_{11} \mathrm{D}_{3} \mathrm{NO}^{+}\right.$expected at $\mathrm{m} / z$ 167.1260; 37 ppm error $)$ in the DART mass spectra. The collision energy was kept constant at $25 \mathrm{eV}$ for both ion sources, but ESI had an additional $25 \mathrm{~V}$ on the 
fragmentor voltage setting to assist the in-source declustering of ions from the ion source. Apparently, the additional $25 \mathrm{~V}$ fragmentor potential for ESI imparts more internal energy to the ions and causes them to enter the collision cell with an elevated internal energy relative to the same ions entering from the DART source.

The main structural fragments of interest are observed in the ESI-generated product ion spectrum at $m / z 149.1160\left(\mathrm{C}_{10} \mathrm{H}_{9} \mathrm{D}_{3} \mathrm{~N}^{+}\right.$expected at $\mathrm{m} / \mathrm{z} 149.1154 ; 6 \mathrm{ppm}$ error $), 134.0960$ $\left({ }^{\circ} \mathrm{C}_{9} \mathrm{H}_{6} \mathrm{D}_{3} \mathrm{~N}^{+}\right.$expected at $\mathrm{m} / \mathrm{z} 134.0920 ; 30 \mathrm{ppm}$ error $), 133.0730\left(\mathrm{C}_{9} \mathrm{H}_{9} \mathrm{O}^{+}\right.$expected at $\mathrm{m} / \mathrm{z}$ 133.0653; $58 \mathrm{ppm}$ error), and $131.0744\left({ }^{\circ} \mathrm{C}_{9} \mathrm{H}_{9} \mathrm{~N}^{+}\right.$expected at $\mathrm{m} / z$ 131.0734; $8 \mathrm{ppm}$ error), which are formed through the loss of $\mathrm{H}_{2} \mathrm{O}$, a methyl radical $\left({ }^{\circ} \mathrm{CH}_{3}\right)$, the $\mathrm{N}$-alkylated moiety and a deuterated methyl radical $\left({ }^{\circ} \mathrm{CD}_{3}\right)$, respectively. The high-resolution of the Q-TOF instrument allows for the determination of elemental formulas for diagnostic ions through their measured accurate masses. The accurate mass measurements of the product ions at $\mathrm{m} / \mathrm{z} 149.1160$ $\left(\mathrm{C}_{10} \mathrm{H}_{9} \mathrm{D}_{3} \mathrm{~N}^{+}\right)$and $\mathrm{m} / z 131.0744\left({ }^{\circ} \mathrm{C}_{9} \mathrm{H}_{9} \mathrm{~N}^{+}\right)$are less than $10 \mathrm{ppm}$ from the exact masses for the proposed structures, whereas the accurate mass measurements for the product ions at $\mathrm{m} / z 134.0960$ $\left({ }^{\circ} \mathrm{C}_{9} \mathrm{H}_{6} \mathrm{D}_{3} \mathrm{~N}^{+}\right)$and $m / z 133.0730\left(\mathrm{C}_{9} \mathrm{H}_{9} \mathrm{O}^{+}\right)$were approximately 3-5 times further from the exact masses for the proposed structures. Even with the expanded error, these sub-60 ppm differences between measured accurate masses and theoretical exact masses provides sufficient confidence to rely on the proposed elemental compositions. Likewise, the mass-dependence of ppm values inflates these reported errors relative to larger molecular weight compounds. If the mass errors discussed above were reported in $\mathrm{mDa}$ as suggested by others [6] our values range from less than $1 \mathrm{mD}$ to $8 \mathrm{mDa}$.

Based on the MS ${ }^{\mathrm{n}}$ analysis on the LIT, the product ions in the ESI-generated product ion spectrum at $m / z 105.0713\left(\mathrm{C}_{8} \mathrm{H}_{9}{ }^{+}\right.$expected at $\mathrm{m} / 2$ 105.0704; $9 \mathrm{ppm}$ error $), \mathrm{m} / \mathrm{z} 103.0575\left(\mathrm{C}_{8} \mathrm{H}_{7}{ }^{+}\right.$ 
expected at $m / z$ 103.0547; $27 \mathrm{ppm}$ error $), m / \mathrm{z} 79.0556\left(\mathrm{C}_{6} \mathrm{H}_{7}{ }^{+}\right.$expected at $\mathrm{m} / \mathrm{z}$. 79.0547; $11 \mathrm{ppm}$ error), and $\mathrm{m} / \mathrm{z} 77.0397\left(\mathrm{C}_{6} \mathrm{H}_{5}{ }^{+}\right.$expected at $\mathrm{m} / \mathrm{z} 77.0391 ; 8 \mathrm{ppm}$ error $)$ originate from the intermediate product ion at $m / z, 133.0730\left(\mathrm{C}_{9} \mathrm{H}_{9} \mathrm{O}^{+}\right)$, whereas the product ions at $\mathrm{m} / z 132.0817$ $\left(\mathrm{C}_{9} \mathrm{H}_{6} \mathrm{D}_{2} \mathrm{~N}^{+}\right.$expected at $\mathrm{m} / \mathrm{z} 132.0780 ; 28 \mathrm{ppm}$ error $)$ and $\mathrm{m} / \mathrm{z} 130.0670\left(\mathrm{C}_{9} \mathrm{H}_{8} \mathrm{~N}^{+}\right.$expected at $\mathrm{m} / \mathrm{z}$ $130.0656 ; 11 \mathrm{ppm}$ error) originate from the intermediate product ions at $m / 2.134 .0960\left({ }^{\circ} \mathrm{C}_{9} \mathrm{H}_{6} \mathrm{D}_{3} \mathrm{~N}^{+}\right)$ and $m / z 131.0744\left({ }^{\circ} \mathrm{C}_{9} \mathrm{H}_{9} \mathrm{~N}^{+}\right)$, respectively. Of all the cathinones studied, the only notable differences between MS/MS data from the DART and ESI ion sources were the abundances of the precursor ion; some residual precursor was always observed for the DART-generated precursors. Likewise, small, but insignificant differences in the accurate mass measurements were observed between the ESI- and DART-generated mass spectra, likely due to recency in mass spectrometer tuning prior to analysis. If needed, the mass accuracy could be improved by the inclusion of internal mass calibrants. However, the internal calibration solution is known to provide several low-mass fragments, such as $m / z 121$ and $m / z 149$, which are also expected in methylenedioxycontaining synthetic cathinones. To prevent possible interference, we deactivated the internal mass calibration in these studies. 

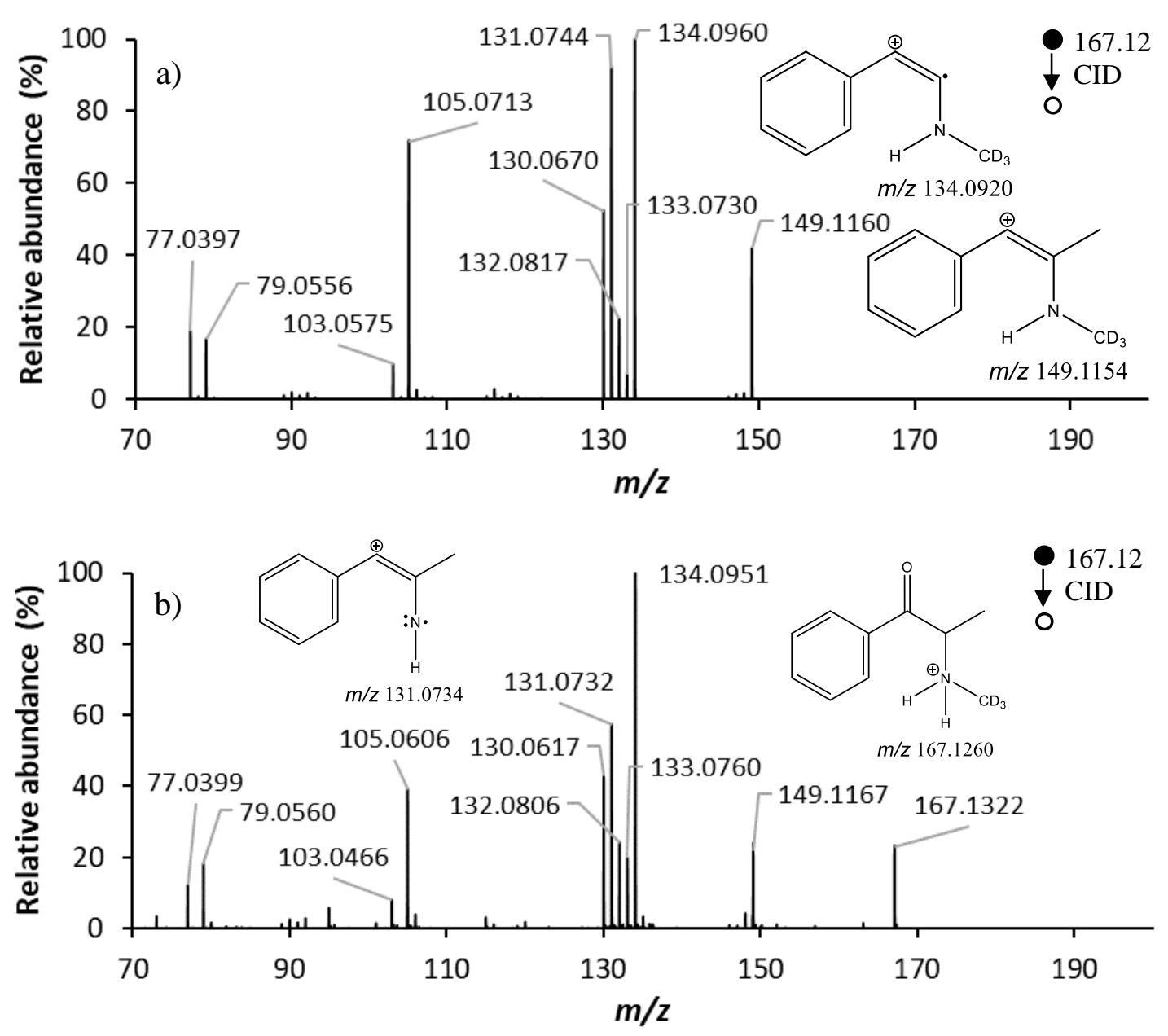

Figure 3.4. Tandem mass spectra of methcathinone- $\mathrm{d}_{3}$ collected on the Q-TOF with both a) ESI and b) DART ionization with a collision energy of $25 \mathrm{eV}$ and skimmer voltage of $65 \mathrm{~V}$. The ESI spectrum was collected with a fragmentor voltage of $175 \mathrm{~V}$, whereas the DART spectrum was collected with a fragmentor voltage of $150 \mathrm{~V}$.

The comparison between the ESI- and DART-generated mass spectra for diethylpropion- $\mathrm{d}_{10}$ (perdeuterated on the diethylpropion moiety) in Figure 3.5 provides further support for the similarity between ESI and DART mass spectra. The accurate mass of the ESI-generated mass spectrum at $m / z 105.0713$ is closer to the exact mass for $\mathrm{C}_{8} \mathrm{H}_{9}{ }^{+}$at $\mathrm{m} / z 105.0704$ (9 ppm error) rather than for $\mathrm{C}_{7} \mathrm{H}_{5} \mathrm{O}^{+}$at $\mathrm{m} / z 105.0340$ (355 ppm error; $37 \mathrm{mDa}$ ). The accurate mass data indicates the primary product ion at $\mathrm{m} / \mathrm{z} 133.0690\left(\mathrm{C}_{9} \mathrm{H}_{9} \mathrm{O}^{+}\right.$expected at $\mathrm{m} / \mathrm{z}, 133.0653 ; 28 \mathrm{ppm}$ error $)$ loses $\mathrm{CO}$ instead of $\mathrm{C}_{2} \mathrm{H}_{4}$ to yield $\mathrm{m} / z 105.0713\left(\mathrm{C}_{8} \mathrm{H}_{9}{ }^{+}\right)$. The product ion at $\mathrm{m} / z 110.1786\left(\mathrm{C}_{6} \mathrm{H}_{4} \mathrm{D}_{10} \mathrm{~N}^{+}\right.$ 
expected at $\mathrm{m} / \mathrm{z}, 110.1743 ; 39 \mathrm{ppm}$ error) is the iminium- $\mathrm{d}_{10}$ ion, whereas the product ion at $\mathrm{m} / \mathrm{z}$ $84.1616\left(\mathrm{C}_{4} \mathrm{H}_{2} \mathrm{D}_{10} \mathrm{~N}^{+}\right.$expected at $\mathrm{m} / \mathrm{z}, 84.1587 ; 38 \mathrm{ppm}$ error $)$ is the diethylamine- $\mathrm{d}_{10}$ ion. As mentioned earlier, diethylpropion- $\mathrm{d}_{10}$ is one of the two $3^{\circ}$ amines analyzed during this study, and the elevated abundance of the product ion at $m / z 105.0713\left(\mathrm{C}_{8} \mathrm{H}_{9}{ }^{+}\right)$shows that the loss of the $N$ alkylated moiety is favored over the loss of $\mathrm{H}_{2} \mathrm{O}$ for $3^{\circ}$ amines. This hypothesis is supported by previous research highlighting the dominant pathway through the loss of water and secondary fragmentation for $2^{\circ}$ amine synthetic cathinones [36].
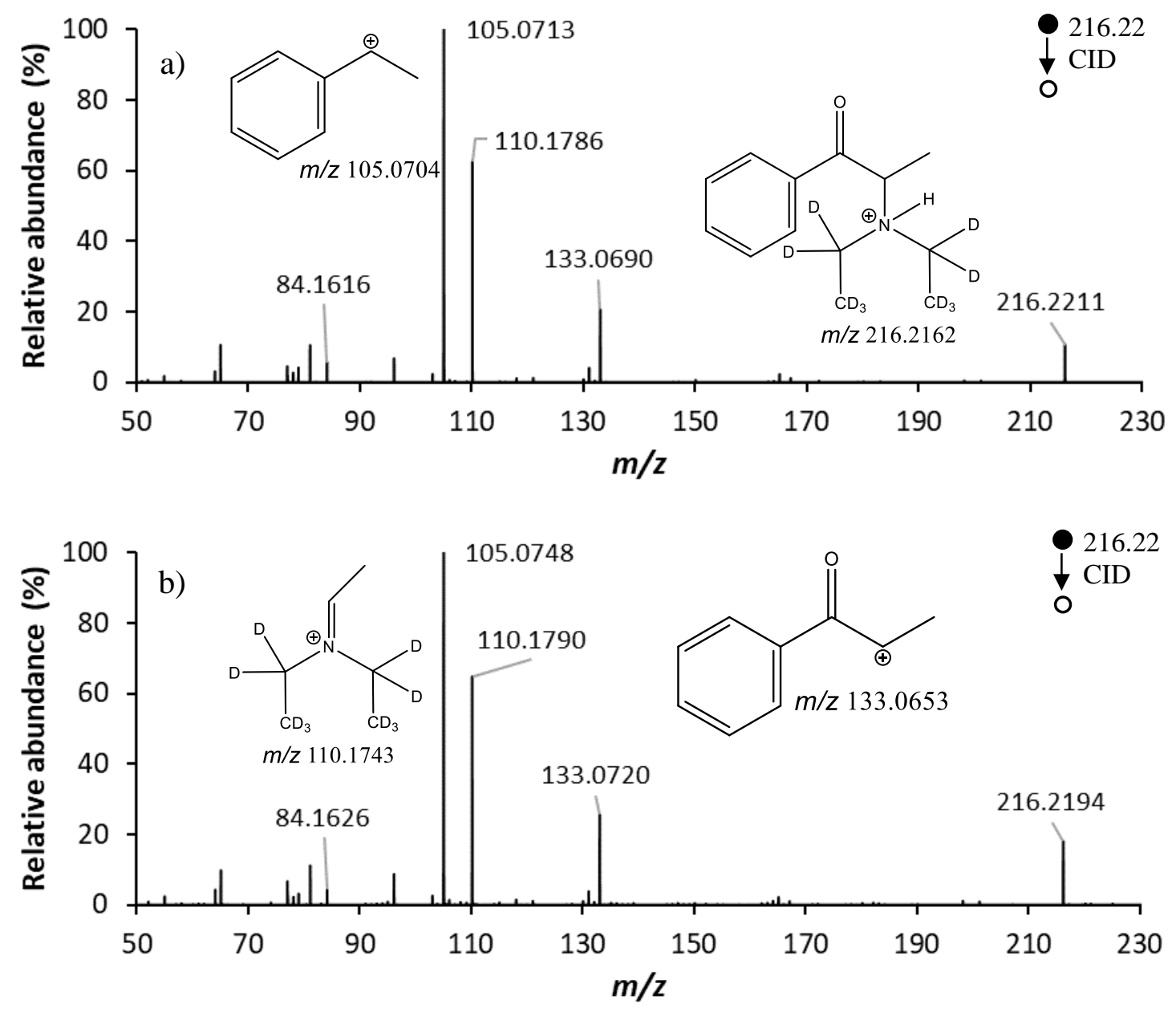

Figure 3.5. Tandem mass spectra of diethylpropion- $d_{10}$ collected on the Q-TOF with both a) ESI and b) DART ionization with a collision energy of $25 \mathrm{eV}$. The ESI spectrum was collected with a fragmentor voltage of $225 \mathrm{~V}$, whereas the DART spectrum was collected with a fragmentor voltage of $150 \mathrm{~V}$, which were both collected with a skimmer voltage of $65 \mathrm{~V}$. 
Figure 3.6 is a comparison between the ESI and DART spectra of eutylone- $\mathrm{d}_{5}$ with the major structural fragments embedded. The ESI-generated product ion spectrum shows the presence of primary product ions at $m / z 223.1516\left(\mathrm{C}_{13} \mathrm{H}_{11} \mathrm{D}_{5} \mathrm{O}_{2} \mathrm{~N}^{+}\right.$expected at $\mathrm{m} / \mathrm{z} 223.1489 ; 12 \mathrm{ppm}$ error), $\mathrm{m} / \mathrm{z} 191.0738\left(\mathrm{C}_{11} \mathrm{H}_{11} \mathrm{O}_{3}{ }^{+}\right.$expected at $\mathrm{m} / \mathrm{z} 191.0708 ; 16 \mathrm{ppm}$ error $), \mathrm{m} / \mathrm{z} 149.0262\left(\mathrm{C}_{8} \mathrm{H}_{5} \mathrm{O}_{3}{ }^{+}\right.$ expected at $\mathrm{m} / \mathrm{z} 149.0238 ; 16 \mathrm{ppm}$ error), and $\mathrm{m} / z 91.1324\left(\mathrm{C}_{5} \mathrm{H}_{7} \mathrm{D}_{5} \mathrm{~N}^{+}\right.$expected at $\mathrm{m} / z$ 91.1278; $51 \mathrm{ppm}$ error) as well as secondary product ions at $\mathrm{m} / \mathrm{z} 194.1161\left({ }^{\circ} \mathrm{C}_{11} \mathrm{H}_{6} \mathrm{D}_{5} \mathrm{O}_{2} \mathrm{~N}^{+}\right.$expected at $\mathrm{m} / \mathrm{z}$ 194.1098; $32 \mathrm{ppm}$ error $), \mathrm{m} / z 193.1442\left(\mathrm{C}_{12} \mathrm{H}_{9} \mathrm{D}_{5} \mathrm{ON}^{+}\right.$expected at $\mathrm{m} / \mathrm{z} 193.1384 ; 30 \mathrm{ppm}$ error $)$, and $m / z 161.0666\left(\mathrm{C}_{10} \mathrm{H}_{9} \mathrm{O}_{2}{ }^{+}\right.$expected at $\mathrm{m} / z 161.0602 ; 40 \mathrm{ppm}$ error). The tertiary product ions at $m / z, 135.0479\left(\mathrm{C}_{8} \mathrm{H}_{7} \mathrm{O}_{2}{ }^{+}\right.$expected at $m / z$ 135.0446; $24 \mathrm{ppm}$ error $)$ and $m / z, 133.0701\left(\mathrm{C}_{9} \mathrm{H}_{9} \mathrm{O}^{+}\right.$ expected at $\mathrm{m} / \mathrm{z} 133.0653 ; 36 \mathrm{ppm}$ error) originate from the secondary product ion at $\mathrm{m} / \mathrm{z} 191.0738$ $\left(\mathrm{C}_{11} \mathrm{H}_{11} \mathrm{O}_{3}{ }^{+}\right)$. The primary product ions at $\mathrm{m} / z 223.1516\left(\mathrm{C}_{13} \mathrm{H}_{11} \mathrm{D}_{5} \mathrm{O}_{2} \mathrm{~N}^{+}\right)$and $\mathrm{m} / z .191 .0738$ $\left(\mathrm{C}_{11} \mathrm{H}_{11} \mathrm{O}_{3}{ }^{+}\right)$form through the loss of $\mathrm{H}_{2} \mathrm{O}$ and the loss of the $N$-alkylated moiety, respectively, whereas the primary product ions at $m / z 149.0262\left(\mathrm{C}_{8} \mathrm{H}_{5} \mathrm{O}_{3}{ }^{+}\right)$and $m / z 91.1324\left(\mathrm{C}_{5} \mathrm{H}_{7} \mathrm{D}_{5} \mathrm{~N}^{+}\right)$ correspond to the methylenedioxy-substituted benzoylium ion and the imminium- $\mathrm{d}_{5}$ ion, respectively. The secondary product ions at $m / z, 194.1161\left({ }^{\circ} \mathrm{C}_{11} \mathrm{H}_{7} \mathrm{D}_{5} \mathrm{O}_{2} \mathrm{~N}^{+}\right)$and $\mathrm{m} / \mathrm{z} 193.1442$ $\left(\mathrm{C}_{12} \mathrm{H}_{9} \mathrm{D}_{5} \mathrm{ON}^{+}\right)$form, respectively, through the loss of an ethyl radical $\left({ }^{\circ} \mathrm{C}_{2} \mathrm{H}_{5}\right)$ and formaldehyde $\left(\mathrm{CH}_{2} \mathrm{O}\right)$ from the intermediate ion at $\mathrm{m} / z, 223.1516\left(\mathrm{C}_{13} \mathrm{H}_{11} \mathrm{D}_{5} \mathrm{O}_{2} \mathrm{~N}^{+}\right)$, whereas the secondary product ion at $m / z \quad 161.0666\left(\mathrm{C}_{10} \mathrm{H}_{9} \mathrm{O}_{2}^{+}\right)$forms through the loss of formaldehyde $\left(\mathrm{CH}_{2} \mathrm{O}\right)$ from the intermediate ion at $m / z 191.0738\left(\mathrm{C}_{11} \mathrm{H}_{11} \mathrm{O}_{3}{ }^{+}\right)$. The error between the accurate mass measurements and the exact masses of the elemental compositions shown were the smallest errors of all possible elemental compositions and within approximately $50 \mathrm{ppm}$, which once again corresponds to $<7$ $\mathrm{mDa}$ error. The HRMS accuracy is sufficient to provide unambiguous elemental compositions and a reasonable indication of the proposed structures. 

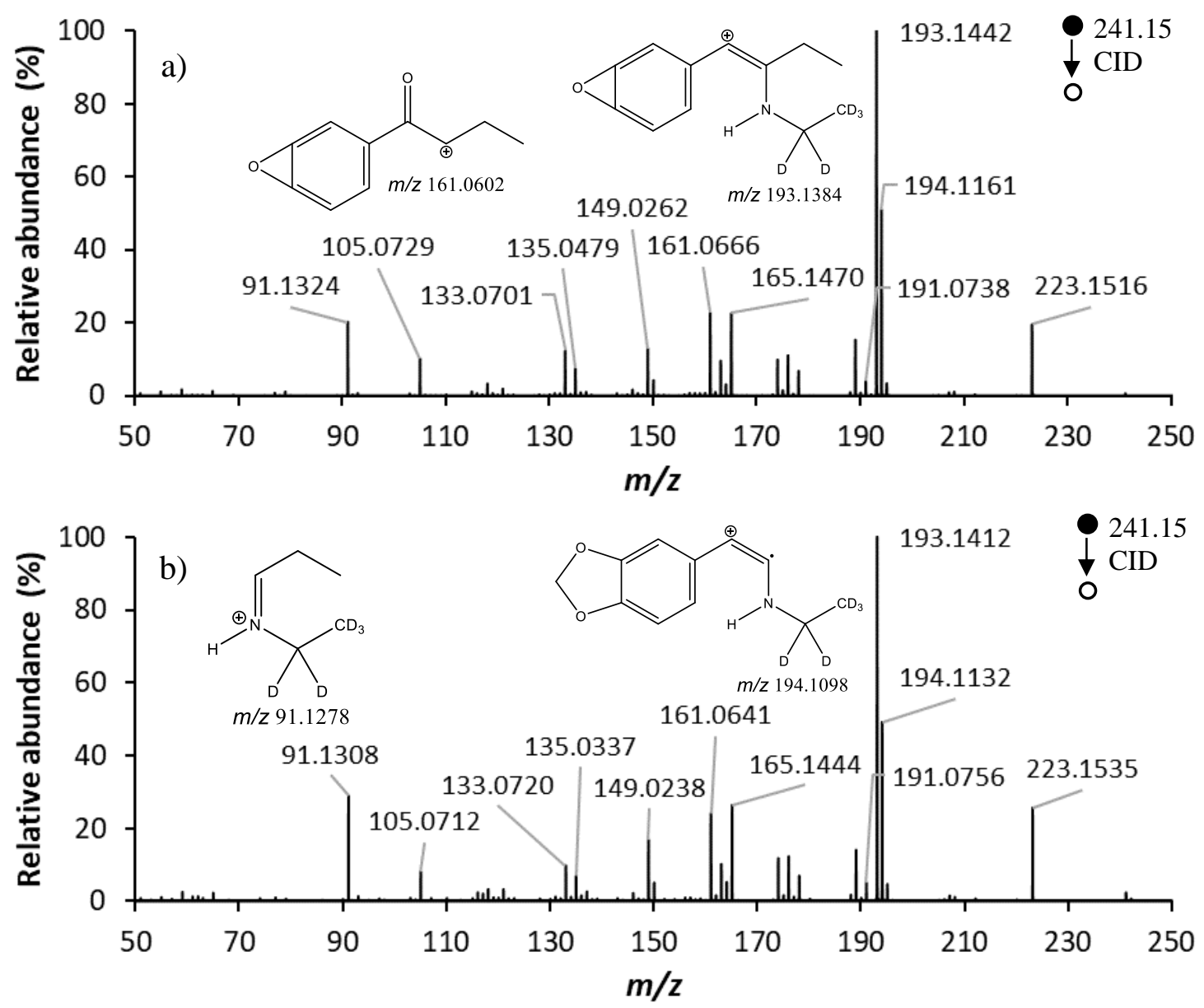

Figure 3.6. Tandem mass spectra of eutylone-d5 collected with both a) ESI and b) DART ionization with a collision energy of $25 \mathrm{eV}$. The ESI spectrum was collected with a fragmentor voltage of $225 \mathrm{~V}$, whereas the DART spectrum was collected with a fragmentor voltage of 150 $\mathrm{V}$, which were both collected with a skimmer voltage of $65 \mathrm{~V}$.

Figure 3.7 shows the proposed fragmentation mechanisms for the $N$-alkylated class of synthetic cathinones, where $\mathrm{X}$ represents substitution to the benzyl moiety and $\mathrm{C}_{\mathrm{n}} \mathrm{H}_{2 \mathrm{n}+1}$ represents varying alkyl chain lengths. The ${ }^{13} \mathrm{C}$ and deuterium labels are not shown in the proposed fragmentation mechanisms, but standards containing these isotopic labels were used to support the proposed mechanisms. 


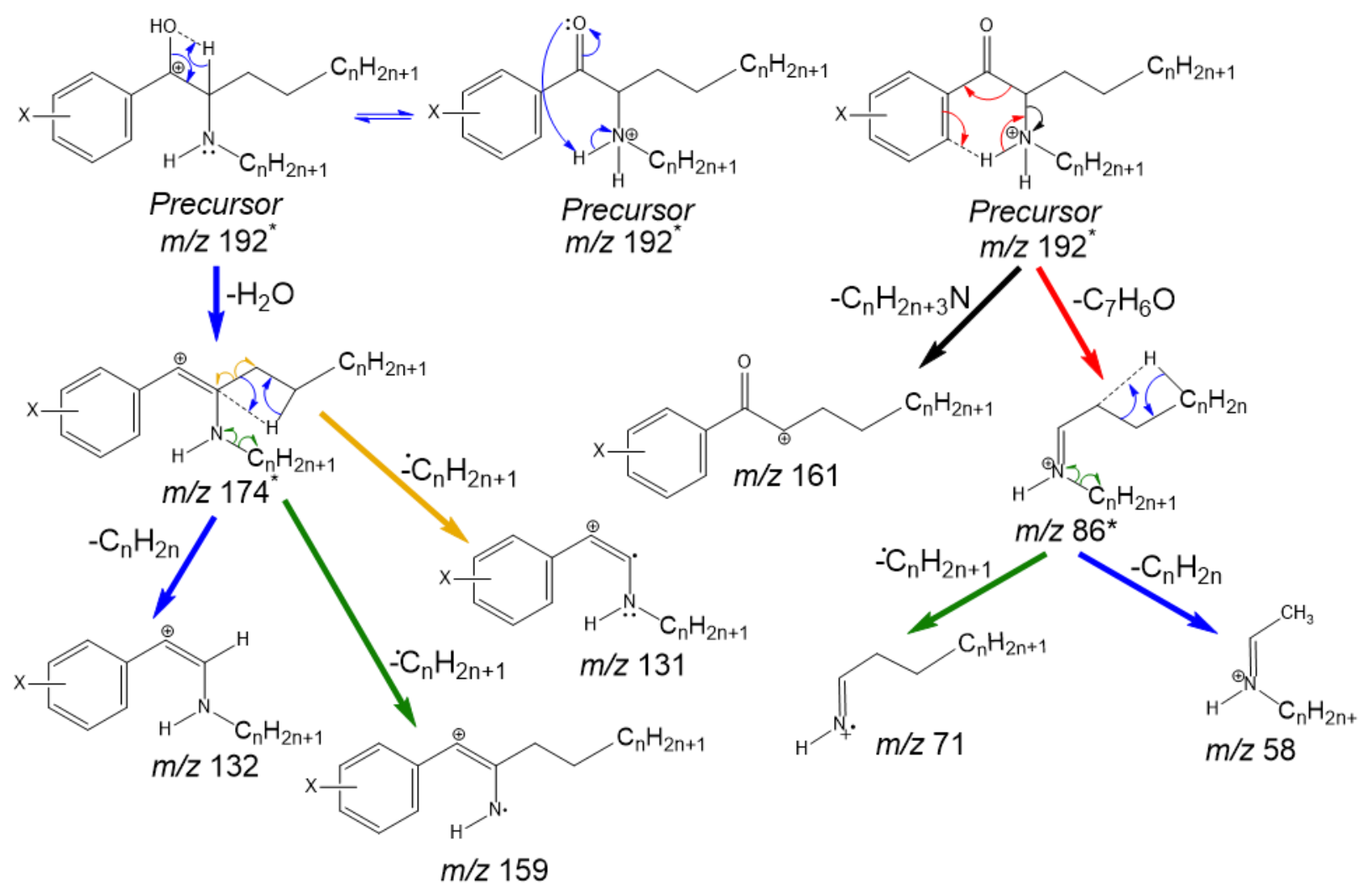

Figure 3.7. Proposed mechanisms for fragmentation pathways of $\mathrm{N}$-alkylated synthetic cathinones. The model compound is pentedrone (aliphatic group $=\mathrm{CH}_{3}, \mathrm{~N}$-alkyl group $=\mathrm{CH}_{3}$, $\mathrm{X}=\mathrm{H}$ ) with ions specific pentedrone indicated with an asterisk (*); different functional groups, $\mathrm{X}$, will shift the product ion $\mathrm{m} / \mathrm{z}$ values accordingly.

The primary fragmentation pathways for the $N$-alkylated class of synthetic cathinones are through the loss of $\mathrm{H}_{2} \mathrm{O}$ (blue pathway), $\mathrm{C}_{n} \mathrm{H}_{2 \mathrm{n}+3} \mathrm{~N}$ (black pathway), and $\mathrm{C}_{7} \mathrm{H}_{6} \mathrm{O}$ (red pathway), which is consistent with previous work by others on this class of compounds $[\underline{15}, \underline{17}, \underline{28}, \underline{37}, \underline{77}$, 78]. The dominant pathway for $2^{\circ}$ amines within this class is through the loss of $\mathrm{H}_{2} \mathrm{O}$ via two hydrogen transfers to the oxygen. Deuterium labeling on the $N$-alkyl group (e.g. methcathinone$\mathrm{d}_{3}$ in Figure 3.4 and eutylone-d5 in Figure 3.6) showed that hydrogens from the $N$-alkyl group do not contribute to the neutral loss of $\mathrm{H}_{2} \mathrm{O}$. Similarly, CID of dibutylone- $\mathrm{d}_{3}$ (alkyl deuterated) provided evidence that the terminal hydrogens do not participate in the water loss. Therefore, the hydrogens must originate from the charging proton and from hydrogen atoms nearer the oxygen, 
as proposed in the 4-center elimination in Figure 3.7, which forms a double bond between the $\alpha$ and $\beta$ carbons to the amine.

Several structures have been proposed for the loss of $\mathrm{H}_{2} \mathrm{O}$ from synthetic cathinones; however, the previous studies did not use of isotopic labeling or HRMS [15, 17, $\underline{28}, \underline{37}, \underline{77}, \underline{78}]$. Our data supports the mechanism proposed in Figure 3.7 based on the loss of a methyl radical $\left({ }^{\circ} \mathrm{CH}_{3}\right)$ from the pentedrone intermediate at $\mathrm{m} / z, 174$ (Figure 3.1b) and the loss of a deuterated methyl radical $\left({ }^{\circ} \mathrm{CD}_{3}\right.$ ) from the methcathinone- $\mathrm{d}_{3}$ intermediate at $\mathrm{m} / \mathrm{z} 149.1160$ (Figure 3.4). In both cases the radical loss would be impossible with an iminium intermediate containing a double bond between the $\mathrm{N}$ and the $N$-alkylated moiety.

Secondary fragmentation also occurs through the loss of alkyl radicals from the aliphatic chain (gold pathway), alkyl radicals from the $N$-alkylated moiety (green pathways), and even-electron alkenes through 4- or 6-center eliminations along the aliphatic chain (blue pathways). The presence of distonic radicals (like the structures at $\mathrm{m} / \mathrm{z} 131$ and $\mathrm{m} / \mathrm{z} 159$ ), along with the loss of $\mathrm{H}_{2} \mathrm{O}$, was observed for every $2^{\circ}$ amine $N$-alkylated synthetic cathinone analyzed in this study.

The second most abundant fragmentation pathway for $2^{\circ}$ amine $\mathrm{N}$-alkylated synthetic cathinones is the formation of the valerophenone-like ion at $m / z, 161$ through the loss of $\mathrm{C}_{\mathrm{n}} \mathrm{H}_{2 \mathrm{n}+3} \mathrm{~N}$ (black pathway). The secondary and tertiary product ions deriving from the valerophenone-like intermediate have been described extensively for $\alpha$-pyrrolidinophenone synthetic cathinones. They include fragments at $m / z 133$ and $m / z$ 119, which have a phthalane-like structure and the tropylium ion at $m / z 91$ [61]. Similar to the $\alpha$-pyrrolidinophenones studied previously [75], which have a facile leaving group in the form of pyrrolidine, fragmentation via the loss of a neutral $3^{\circ}$ amines was the dominant fragmentation pathway for the $3^{\circ}$ amines analyzed here. The other observed fragmentation pathway for $2^{\circ}$ and $3^{\circ}$ amines is through the loss of $\mathrm{C}_{7} \mathrm{H}_{6} \mathrm{O}$ (red pathway) to form 
iminium ions similar to the one at $\mathrm{m} / \mathrm{z} 86$ for pentedrone in Figure 3.7. This pathway is readily explained via a McLafferty rearrangement and leads to secondary fragments through the loss of alkyl radicals from the $N$-alkyl chain (green pathway) and even-electron alkenes through 4-center eliminations from the aliphatic chain (blue pathway).

\subsubsection{GC-EI-MS}

Previous literature on the analysis of phenethylamines with GC-EI-MS has documented the need to identify phenethylamines, their metabolites and their thermal degradation products, the latter of which can occur in the injection port or on-column and are often observed as a shoulder or split peak in the GC [62-64]. The 2,3-enamine degradation products observed from the $N$ alkylated synthetic cathinones in this project were always minor relative to the abundance of the precursor compound. Figure 3.8 shows the GC-EI-MS spectrum of ${ }^{13} \mathrm{C}$-ethylone, which is isotopically labeled with on the carbonyl carbon. The extensive fragmentation caused by EI ionization occurs through well-established fragmentation mechanisms such as the formation of the benzoylium ion at $m / z, 105$ or iminium ion $\left(\mathrm{C}_{n} \mathrm{H}_{2 n+2} \mathrm{~N}^{+}\right)$at $m / z 72$ for ethylone [15]. The established fragmentation mechanisms for EI-MS are both radical-directed and charge-directed, in contrast to the charge-remote, 4-center eliminations commonly observed in protonated tandem mass spectra. The iminium ions are so dominant in EI that competing fragmentation pathways are of low abundance ( $<10 \%$ base peak) and provide minimally informative spectra [79]. Figure 3.8 contains the embedded major structural fragments. The spectrum has been truncated to $m / z 40-200$ because the molecular ion at $\mathrm{m} / \mathrm{z} 221$ was not observed. The most abundant structural fragments are the methylenedioxy-substituted benzoylium and phenylium ions at $m / z 150$ and 121 , respectively, and the iminium ion observed at $m / z$ 72. Note that the methylenedioxy-substituted tropylium ion at $m / z$ 135 requires extensive rearrangements. 


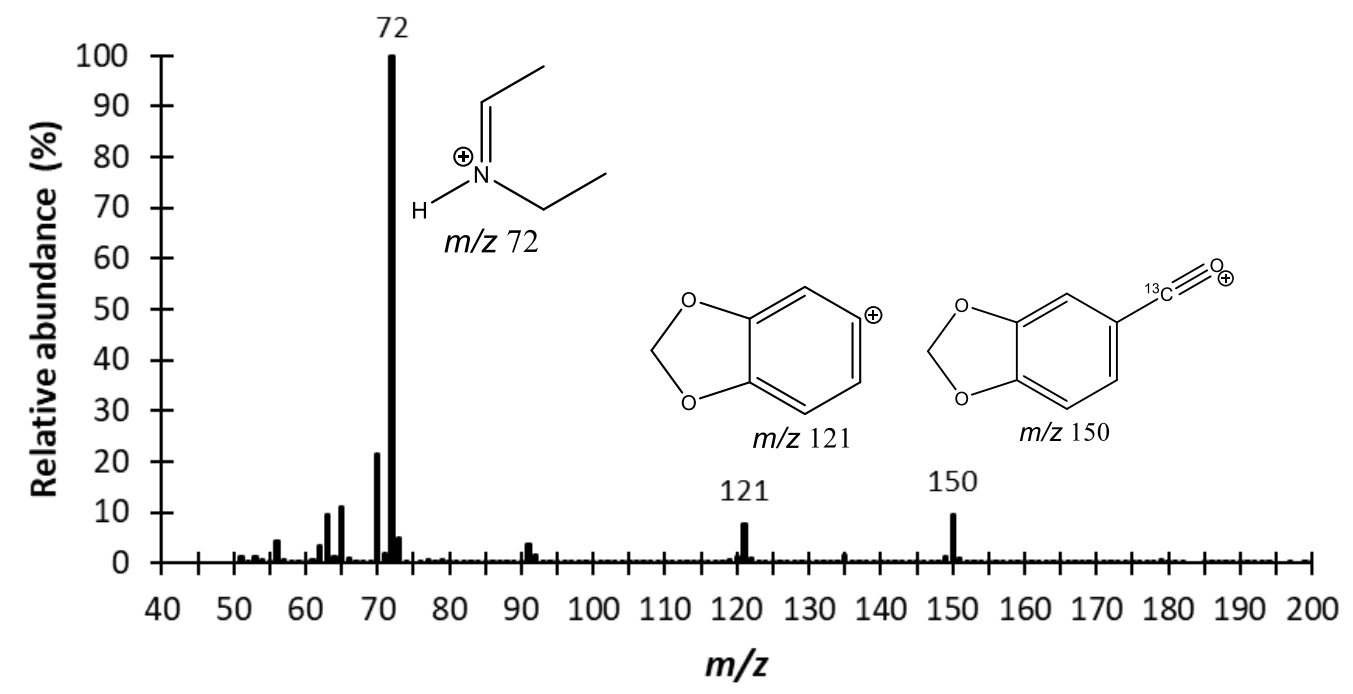

Figure 3.8. Full scan mass spectra of ${ }^{13} \mathrm{C}$-ethylone (isotopically labeled with on the carbonyl carbon) collected with GC-EI-MS.

The GC-EI-MS spectrum of $\alpha$-propylaminopentiophenone (Figure 3.9) follows the same fragmentation behavior as ${ }^{13} \mathrm{C}$-ethylone, with the formation of the iminium, benzoylium and phenylium ions of significant abundance. The molecular ion at $m / z 219$ is not observed, and the fragmentation pattern is consistent with previous work [77]. However, $\alpha$ propylaminopentiophenone has a propyl amine and pentyl aliphatic chain, leading to the formation of the iminium ion at $m / z 114$ in Figure 3.9. This iminium ion can fragment further to form another iminium ion at $\mathrm{m} / \mathrm{z}, 72$ through the loss of a propylene neutral. 


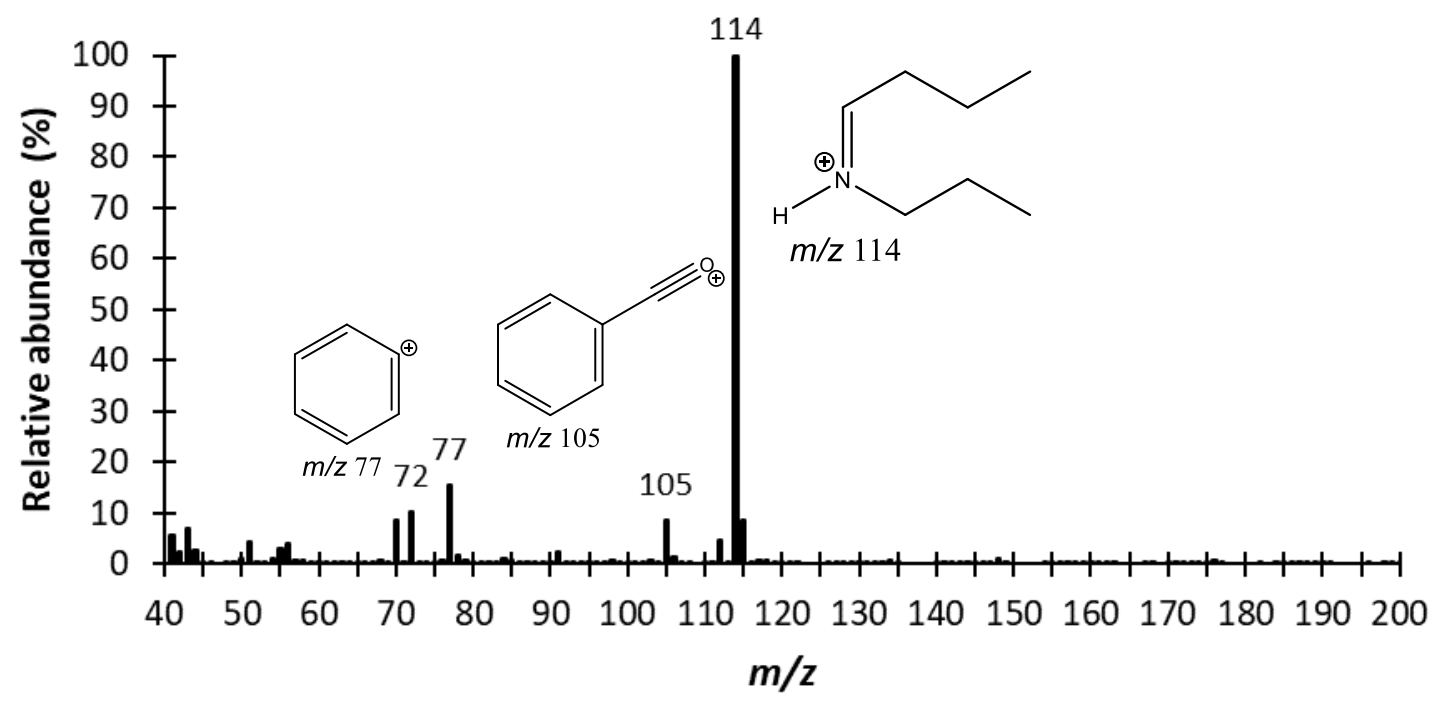

Figure 3.9. Full scan mass spectra of $\alpha$-propylaminopentiophenone collected with GC-EI-MS.

Figure 3.10 shows the GC-EI-MS spectrum of ${ }^{13} \mathrm{C}$-benzedrone-isotopically labeled on the carbonyl carbon — with the major structural fragments embedded. This compound is unique in that the $N$-alkylated moiety contains an aromatic ring. The aromatic ring causes a notable shift in the fragmentation behavior of this compound. For example, the base peak of Figure 3.10 is the tropylium ion at $\mathrm{m} / \mathrm{z}, 91$, which forms through charge-directed cleavage of the aromatic moiety. The aromatic substituted iminium ion $(\mathrm{m} / \mathrm{z} 134),{ }^{13} \mathrm{C}$-labled methyl substituted benzoylium ion $(\mathrm{m} / \mathrm{z}, 120)$, and cyclopentadiene ion $(\mathrm{m} / \mathrm{z}, 65)$ are structural ions of significant abundance. 


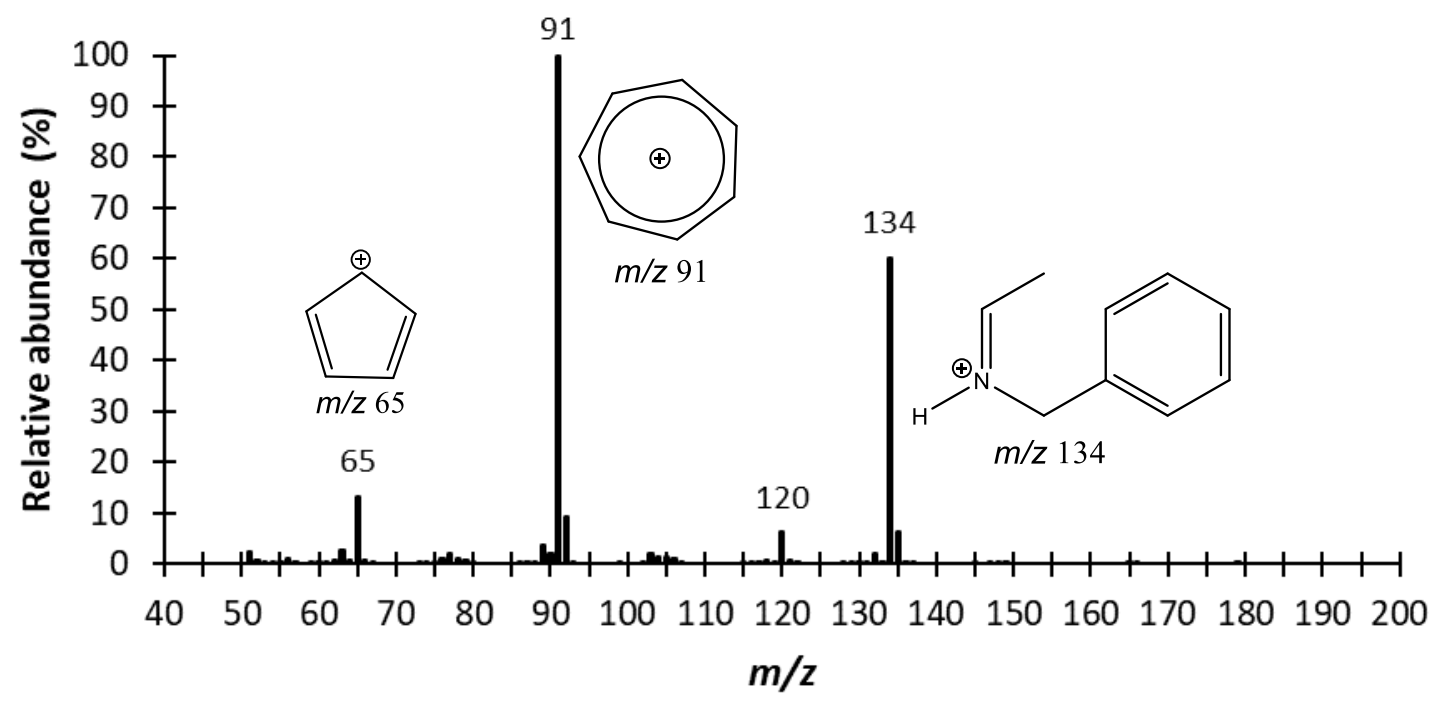

Figure 3.10. Full scan mass spectra of ${ }^{13} \mathrm{C}$-benzedrone collected with GC-EI-MS.

Figure 3.11 contains the proposed EI-MS fragmentation mechanisms for the $N$-alkylated class of synthetic cathinones, where $X$ represents substitution to the benzyl moiety and $\mathrm{C}_{n} \mathrm{H}_{2 n+1}$ represents varying alkyl chain lengths. Pentedrone is the model compound for illustrative purposes. The isotopic labels are not shown in Figure 3.11 but were used to further support the proposed mechanisms. The acylium (red pathway) and iminium ions (blue pathway) are the dominant fragmentation behavior for $N$-alkylated synthetic cathinones. The acylium ion pathway is initiated by $\alpha$-cleavage of the bond between the carbonyl carbon and the $\alpha$-carbon adjacent to the amine. The subsequent loss of $\mathrm{CO}$ from the acylium ion produces the phenylium ion at $\mathrm{m} / \mathrm{z} 77$, which can then ring-contract to lose $\mathrm{C}_{2} \mathrm{H}_{2}$ to form the cyclobutadienyl cation at $\mathrm{m} / z 51$. The iminium ion cascade is initiated by a radical electron on the nitrogen and $\alpha$-cleavage of the bond between the carbonyl carbon and the $\alpha$-carbon adjacent to the pyrrolidine ring. The iminium ion pathway forms secondary fragmentation through a 4-center elimination along the alkyl chain (black pathway) and radical-directed cleavage (gold pathway). The benzoylium ion can also form 
through $\alpha$-cleavage of the bond between the carbonyl carbon and the $\alpha$-carbon, which is initiated by a lone pair of electrons on the oxygen (green pathway).
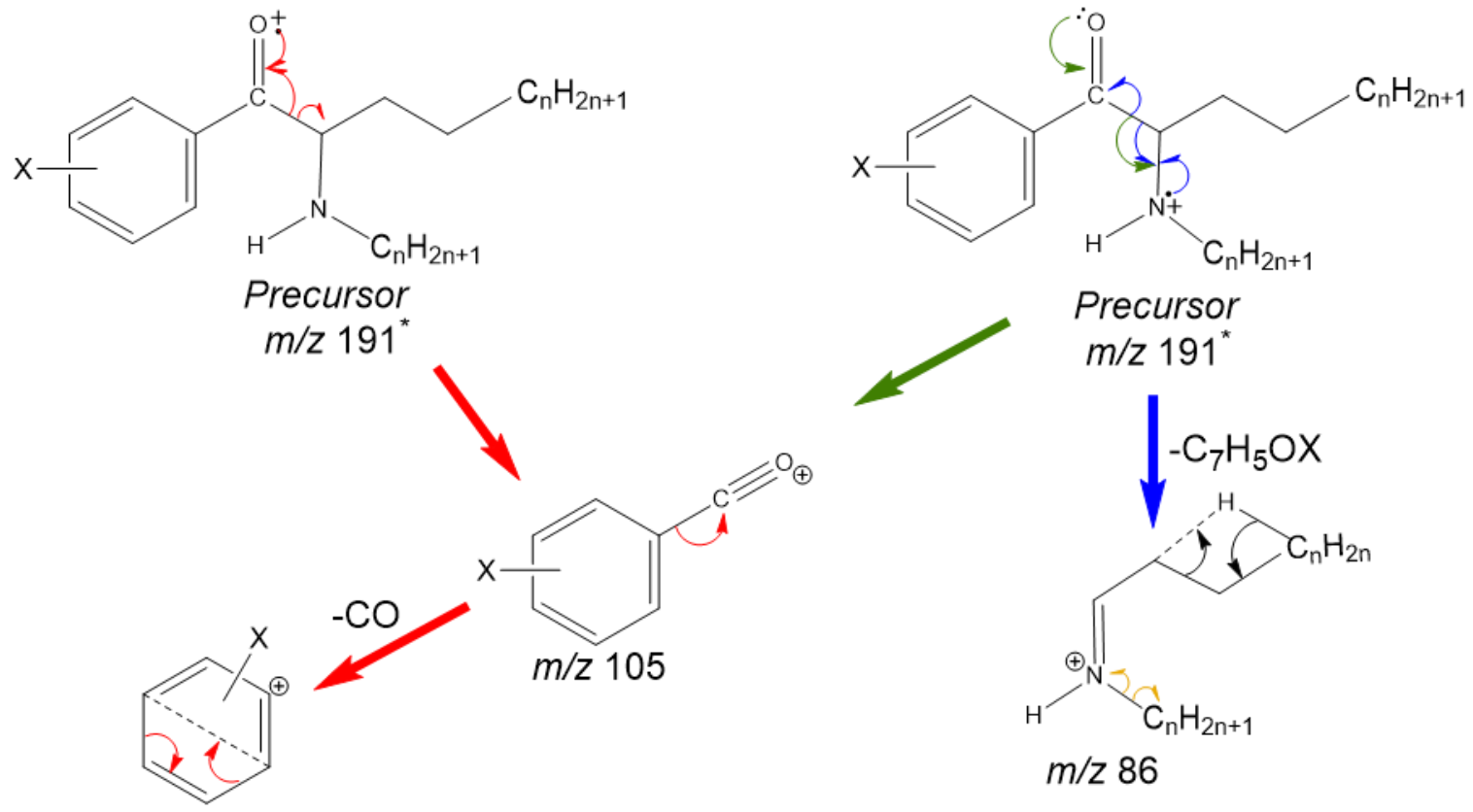

Precursor $m / z 191^{*}$

$m / z 77$
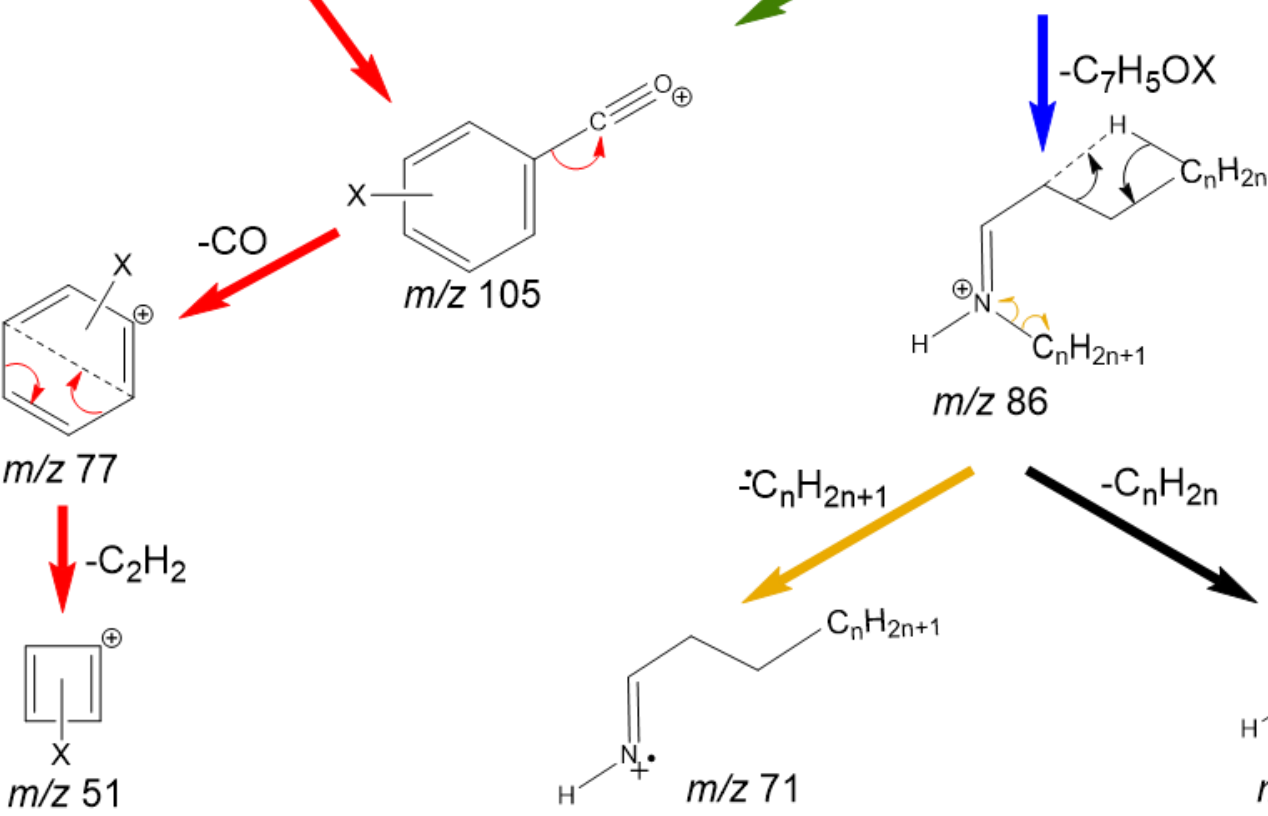

$m / z 71$

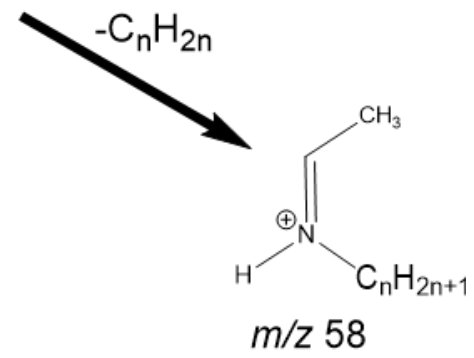

Figure 3.11. Proposed EI-MS fragmentation mechanisms for the $N$-alkylated class of synthetic cathinones (adapted from $[\underline{60}, \underline{75}]$ ). The model compound is pentedrone (aliphatic group $=\mathrm{CH}_{3}$, $\mathrm{N}$-alkyl group $=\mathrm{CH}_{3}, \mathrm{X}=\mathrm{H}$ ) with ions specific to pentedrone indicated with an asterisk (*).

\subsection{Conclusions}

The combination of isotopic labeling, $\mathrm{MS}^{\mathrm{n}}$, and HRMS was used to further develop the current understanding of the fragmentation behavior of the $\mathrm{N}$-alkylated class of synthetic cathinone derivatives. In addition, a comparison between three different ionization and fragmentation techniques commonly available in crime laboratories provides insight into the fragmentation 
behavior of these compounds under different instrumental conditions. The most common mass spectrometer used for seized drug analysis is GC-EI-MS, whereas the toxicological community typically employs LC-ESI-MS/MS and more recently the community has expanded the use of ambient ionization, such as DART. The identification of characteristic fragmentation pathways provides practitioners with an additional tool for the identification of $N$-alkylated synthetic cathinones.

The protonated tandem mass spectrometry fragmentation pathways and proposed fragmentation mechanisms were developed based on the analysis of a series of $N$-alkylated synthetic cathinone derivatives. The LIT instrument provided the capability to identify the direct relationship between each ion along the proposed fragmentation pathways using $\mathrm{MS}^{\mathrm{n}}$ analysis. In comparison, the Q-TOF mass spectrometer identified the elemental formulas by comparing the accurate mass measurements with the theoretical exact mass measurements. The identification of fragmentation pathways that are conserved between ion trap- and beam-type mass spectrometers provides additional confidence, although the multi-collisional environment of the ion trap tends to favor low-energy pathways and higher-mass ions [35]. Finally, both ESI and DART ion sources produced in-tact protonated molecular ions with very similar protonated tandem mass spectra for all the analogs studied.

The diagnostic ions formed with protonated tandem mass spectrometry occur through the loss of $\mathrm{H}_{2} \mathrm{O}, \mathrm{C}_{\mathrm{n}} \mathrm{H}_{2 \mathrm{n}+3} \mathrm{~N}$, and $\mathrm{C}_{7} \mathrm{H}_{6} \mathrm{O}$, as displayed in Figure 3.7. The loss of $\mathrm{H}_{2} \mathrm{O}$ appears to be the dominant pathway for $2^{\circ}$ amines, which is in stark contrast to $3^{\circ}$ amines, which favors the formation of alkylphenones via the loss of imine neutrals. The hydrogens lost as water do not originate from the $N$-alkyl groups or the terminal carbon of the alkyl chains, but instead originate from the protonating hydrogen and an $\mathrm{H}$ atom nearer to the carbonyl group. The formation of 
characteristic secondary fragmentation for $2^{\circ}$ amines occurs through the loss of alkenes $\left(\mathrm{C}_{n} \mathrm{H}_{2 n}\right)$ from the amine moiety and alkyl radicals $\left({ }^{\circ} \mathrm{C}_{\mathrm{n}} \mathrm{H}_{2 \mathrm{n}+1}\right)$ from both the amine and aliphatic chains. The same fragmentation behavior is observed for the iminium ion fragmentation pathway through the loss of alkyl radicals $\left({ }^{\circ} \mathrm{C}_{\mathrm{n}} \mathrm{H}_{2 \mathrm{n}+1}\right)$ and alkenes $\left(\mathrm{C}_{\mathrm{n}} \mathrm{H}_{2 \mathrm{n}}\right)$ from the amine moiety.

In contrast to CID of protonated precursors, EI-MS spectra of $N$-alkylated synthetic cathinones are dominated by radical-directed cleavages that lead to acylium and iminium ions. One downside to the conserved iminium ions is when one observes, say, a 42-Da shift in the iminium ion from ethylone to $\alpha$-propylaminopentiophenone one cannot readily confirm whether the three additional methylene groups are on the aliphatic chain, the $N$-alkyl chain or a combination of the two.

The proposed fragmentation mechanisms for the protonated tandem mass spectrometry and EI-MS pathways provide rational explanations for the observed fragmentation behavior based on the combination of isotopic labeling, MS ${ }^{\mathrm{n}}$, HRMS, and EI-MS. The significance of identifying fragmentation mechanisms is the application of this knowledge to novel compound identification. The interrogation of collected mass spectra against the proposed fragmentation pathways provides an additional tool for the identification of novel $\mathrm{N}$-alkylated synthetic cathinones. Finally, providing fragmentation pathways and mechanistic explanations that are applicable across a range of ionization and fragmentation conditions provides useful multi-discipline mass spectral interpretation to practitioners. 


\section{Chapter 4: The characterization of isobaric product ions of fentanyl using multi-stage mass spectrometry, high-resolution mass spectrometry and isotopic labeling}

Reproduced in part with permission from J.T. Davidson, Z.J. Sasiene, G.P. Jackson, Drug Testing and Analysis, DOI: 10.1002/dta.2758.

\subsection{Introduction}

Fentanyl and fentanyl analogs have emerged as some of the most deadly compounds from the growing opioid crisis in America [80]. Fentanyl is a synthetic opioid approved by the FDA as an analgesic and anesthetic, but licit and illicit fentanyl analogs end up on the drug market through theft, fraudulent prescriptions and illicit distribution by patients, doctors, and pharmacists [81]. Since 2013, fentanyl and fentanyl analogs have become increasingly common adulterants in heroin seizures, and their incredible efficacy for binding to opioid receptors in the body has caused accidental overdoses in almost every state [82]. According to the 2017 National Laboratory Forensic Information System (NFLIS), more than 56,000 fentanyl drug reports were filed the year before by local and state forensic laboratories [83].

The Janssen and Siegfried methods are the two main synthetic routes for clandestine synthesis of fentanyl [84]. The Siegfried method is the easier of the two approaches, and it uses $N$-phenethyl4-piperidone (NPP) as the starting material and produces 4-anilino- $N$-phenethylpiperidine (4ANPP) as an intermediate to the fentanyl product. Investigators can benefit from knowing the synthetic route of a fentanyl seizure, and the synthetic pathway can be established by the identification of residual unreacted precursors in the seizure. The Siegfried method uses 4-ANPP and the Janssen method uses benzylfentanyl. According to the DEA, four of the five domestic fentanyl clandestine labs seized since 2000 used the Siegfried method or a modified version thereof [84]. 
Whereas fentanyl and its synthetic precursors are Schedule II narcotics, the impact on public health has led fentanyl analogs such as $\alpha$-methylfentanyl, 3-methylfentanyl, acetylfentanyl, butyryl fentanyl, and $\beta$-hydroxythiofentanyl to be listed as Schedule I narcotics $[\underline{82}, \underline{84}, \underline{85}]$. Figure 4.1 shows the generic chemical structure of fentanyl analogs. This core structure is conserved in almost all analogs. Para-methylphenethylacetylfentanyl is an example of a fentanyl analog generated through modification at location R1. Thiofentanyl, $\alpha$-methylfentanyl, and $\beta$-hydroxyfentanyl are examples of fentanyl analogs generated through modification at location R2. Carfentanil and 3methylfentanyl are examples of modifications at location R3. Sufentanil, alfentanil and remifentanil all contain modifications at locations R2 and R3, where R3 refers to a substitution at any position on the piperidine ring. Alteration in the length of the aliphatic chain at location R4 differentiates butyrylfentanyl and acetylfenantyl analogs from fentanyl. Finally, substitutions such as a fluorine at location R5 generate fentanyl analogs such as para-fluorofentanyl [86].

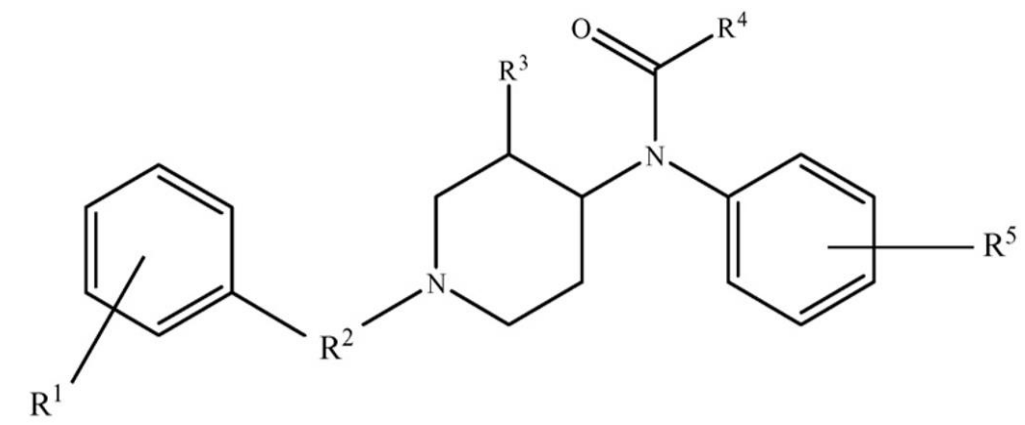

Figure 4.1 [7]. Generic chemical structure of fentanyl highlighting regions of substitutions for fentanyl analogs.

The differentiation of fentanyl analogs with electron ionization (EI) mass spectrometry has proven to be difficult. Mallette et al. [88] noted that 2-methylfentanyl and 3-methylfentanyl can only be distinguished based on the subtle differences in the relative abundance of ions at $m / z 202$, 203, 160 and 216. Similarly, cyclopropylfentanyl and crotonylfentanyl are only distinguishable by differences in the relative abundance of ions at $\mathrm{m} / \mathrm{z}, 69$ and $\mathrm{m} / \mathrm{z}, 105$ [89]. Kanamori et al. reported 
the conserved nature of fragmentation pathways with 3-methylfentanyl analogs with all spectra having a base peak due to the benzyl cleavage [90]. The relative ion abundances were similar between compounds, with cis-isomers having larger abundances than trans-isomers, such as $\mathrm{m} / \mathrm{z}$ 216, 203, 160, and 105 for cis-3-methylfentanyl relative to trans-3-methylfentanyl. However, some spectra, such as the diastereomers of $\beta$-hydroxy-cis-3-methylfentanyl, were too similar to differentiate. Ohta et al. have reported on the highly conserved nature of fentanyl analogs and called for forensic science laboratories to prepare for new designer drugs [91]. The conserved fragmentation pathways of these common structures has enabled the National Institute of Standards and Technology (NIST) to develop an EI-MS mass spectral database and algorithm specifically designed to assist with the identification of novel opioids including fentanyl analogs [92].

Whereas the use of tandem mass spectrometry is one of the most effective ways to identify fentanyl analogs, especially at trace levels in toxicological and seized drug samples [ $\underline{93}, \underline{94}]$, certain analogs are extremely difficult to distinguish. For example, Feasel et al. observed largely analogous collision-induced dissociation (CID) spectra between carfentanil and remifentanil [95]. Caspar et al. discussed the value of having group-indicating ions that might help to identify novel compounds [96]. They recommended HRMS to help identify the elemental compositions of different fragment ions, including the fragment at $m / z 188.1439$ in the case of fentanyl analogs [96]. However, Wichitnithad et al. observed at least two isobaric product ions with the same exact mass at $m / z 188.1439$ [97]. They were only able to distinguish the constitutional isomers using $\mathrm{MS}^{3}$ and deuterium labeling [97]. Their observation further highlights the need for a better understanding of the fragmentation pathways of fentanyl and fentanyl analogs. 
Two approaches that have demonstrated success in the identification of novel psychoactive substances (NPS) are the use of multi-stage mass spectrometry $\left(\mathrm{MS}^{\mathrm{n}}\right)[98,99]$ and the use of accurate mass measurements with high-resolution mass spectrometry (HRMS) [100, 101]. The current work employs both of these tactics and isotopic labeling to elucidate additional fragmentation mechanisms of fentanyl and 4-ANPP. The discovery of three isobaric product ions at $\mathrm{m} / \mathrm{z} 188$ adds to the understanding of the fragmentation behavior of fentanyl analogs and helps to defend the use of transitions such as $m / z 337 \rightarrow m / z 188$ for the quantitation and identification of fentanyl in seized drugs and biological fluids [7, 102-104]. The mechanism of formation of the new intermediate can be extrapolated to other fentanyl analogs to help explain some previously unidentified product ions in the spectra of existing and future fentanyl analogs.

\subsection{Methods}

\subsubsection{Sample Preparation}

The 4-ANPP fentanyl precursor, fentanyl, and fentanyl- $\mathrm{d}_{5}$ (deuterated around the aniline) standards were purchased through Cayman Chemical (Ann Arbor, MI, USA). The 4-ANPP and fentanyl standards were dissolved in a solution of 49\% HPLC grade methanol, 49\% distilled water, and $2 \%$ acetic acid. The fentanyl- $\mathrm{d}_{5}$ certified reference material (CRM) was left in the original methanol solvent to reduce the risk of hydrogen back exchange. The HPLC grade methanol was supplied by Fisher Scientific (Palo Alto, CA, USA) and the acetic acid was supplied by Acros Organics (Palo Alto, CA, USA). 


\subsubsection{Instrumentation}

\subsubsection{Thermo Scientific Velos Pro Linear Ion Trap (LIT)}

A Thermo Scientific Velos Pro Linear Ion Trap (LIT) mass spectrometer was operated with a heated-electrospray ionization (HESI) source. The HESI source was operated at $50{ }^{\circ} \mathrm{C}$ with a spray voltage of 4,000 V. The nitrogen sheath gas was operated at 8 arbitrary units with a nitrogen auxiliary gas flow of 5 arbitrary units. The mass spectrometer capillary temperature was $275{ }^{\circ} \mathrm{C}$. The scan range and normalized collision energies (NCEs) were compound specific and are labeled with each mass spectrum. An isolation width of $1 \mathrm{Da}$ was used for all samples. Ultra-pure helium was used as the bath gas purchased through Matheson Tri-Gas (Fairmont, WV, USA).

\subsubsection{Agilent Technologies 6538 UHD Accurate-Mass Quadrupole Time-of-Flight (Q-TOF)}

An Agilent Technologies 6538 UHD Accurate-Mass Quadrupole Time-of-flight (Q-TOF) mass spectrometer was operated with dual electrospray ionization (ESI) with a spray voltage of $3,500 \mathrm{~V}$. The nitrogen gas was set to $300{ }^{\circ} \mathrm{C}$ with a drying gas flow of $5 \mathrm{~L} / \mathrm{min}$ and a nebulizer flow of 30 psig. The MS fragmentor and skimmer voltages were operated at $225 \mathrm{~V}$ and $65 \mathrm{~V}$, respectively. The scan range and collisions energies were specific to each compound and are labeled in each mass spectrum. An isolation width of 1.3 Da was used for all samples. Ultra-pure nitrogen was used for the collision gas purchased through Matheson Tri-Gas (Fairmont, WV, USA).

\subsubsection{Data Analysis}

Xcalibur 2.0.0.48 software was used for the Velos Pro LIT data analysis, whereas MassHunter Qualitative Analysis B.05.00 was used for the Agilent Q-TOF data analysis. Microsoft Excel version 14 (Microsoft, Redmond, WA, USA) and ChemDraw 16.0 (PerkinElmer, Waltham, MA, USA) were used for the mass spectral plots and mass spectral fragmentation mechanisms. 


\subsubsection{Mass Spectral Interpretation and Mechanisms}

The fragmentation mechanisms for each compound are based on $\mathrm{MS}^{\mathrm{n}}$ analyses, accurate mass measurements and prevailing electron pushing conventions. The proposed mechanisms follow the expected lowest energy pathways [35]. Whereas the identification of the exact hydrogen(s) involved in a specific rearrangement was not possible in this study, we could exclude certain hydrogen atoms by using perdeuterated analogs. Examples include $\mathrm{MS}^{\mathrm{n}}$ of fentanyl that was perdeuterated on the aniline ring. $\mathrm{MS}^{\mathrm{n}}$ resolved the relationships between primary, secondary and tertiary product ions in a variety of pathways, and even though the exact atoms could not always be resolved in a rearrangement, the results provided a deeper level of understanding than the present status.

\subsection{Results/Discussion}

Figure 4.2 shows an example of the product ion spectrum generated from the fragmentation of the $[\mathrm{M}+\mathrm{H}]^{+}$protonated precursor of fentanyl observed at $\mathrm{m} / z, 337$ with the major structural fragments embedded. The base peak in this spectrum is observed at $m / z 188$, which has been identified as a characteristic ion for the identification of fentanyl derivatives [96]. However, based on the competing mechanisms for the loss of the $N$-phenylpropanamide neutral, previous literature has demonstrated that this peak must contain at least two isobaric product ions with different constitutional structures [97]. 


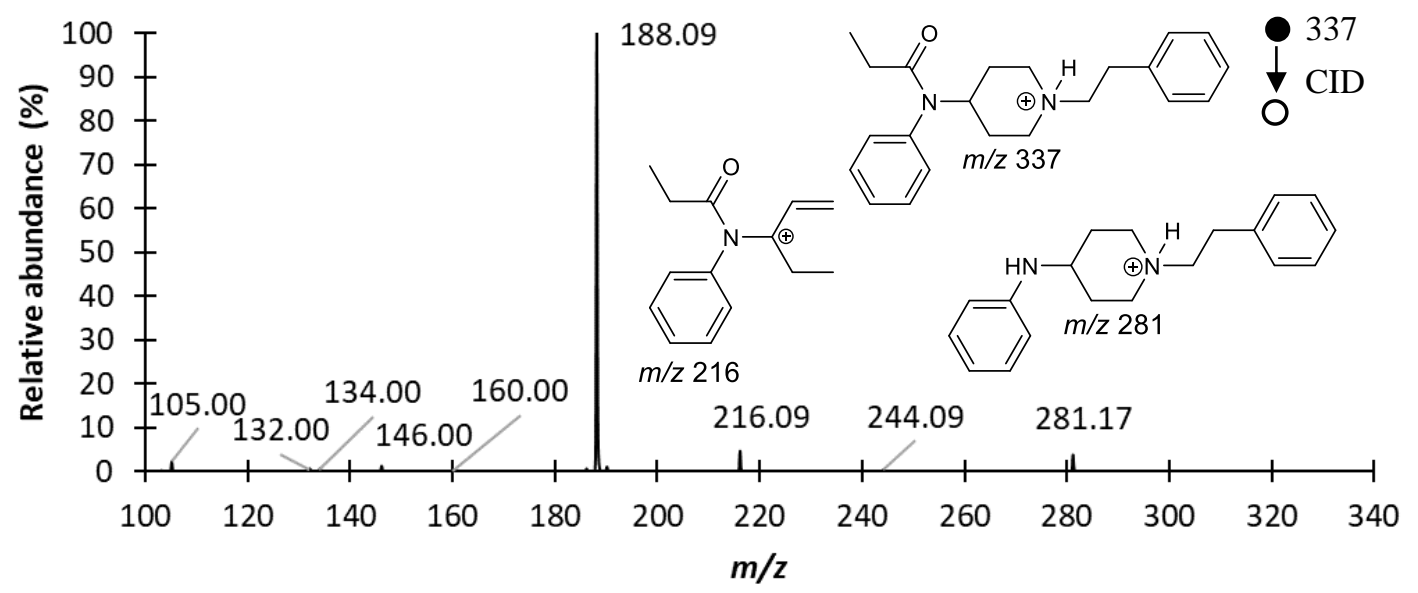

Figure 4.2. Tandem mass spectrum of protonated fentanyl using CID in a linear ion trap mass spectrometer (35\% NCE).

Other ions of significant abundance are observed at $\mathrm{m} / \mathrm{z} 281$ and $\mathrm{m} / \mathrm{z} 216$, of which the ion at $\mathrm{m} / \mathrm{z} 281$ has been shown to fragment into $\mathrm{m} / \mathrm{z} 188[\underline{94}, \underline{98}, \underline{105}]$. Figure 4.3 shows the $\mathrm{MS}^{3}$ product ion spectrum from the isolation and fragmentation of the intermediate product ion at $\mathrm{m} / \mathrm{z} 281$ (Figure 4.3a) and the $\mathrm{MS}^{4}$ product ion spectrum of the isolation and fragmentation of the intermediate product ion at $\mathrm{m} / \mathrm{z} 188$ from Figure 4.3a. These spectra contain the major structural fragments for each MS-level. The distribution of product ions in Figure 4.3b is in agreement with the work of Wichitnithad et al. [97]. 

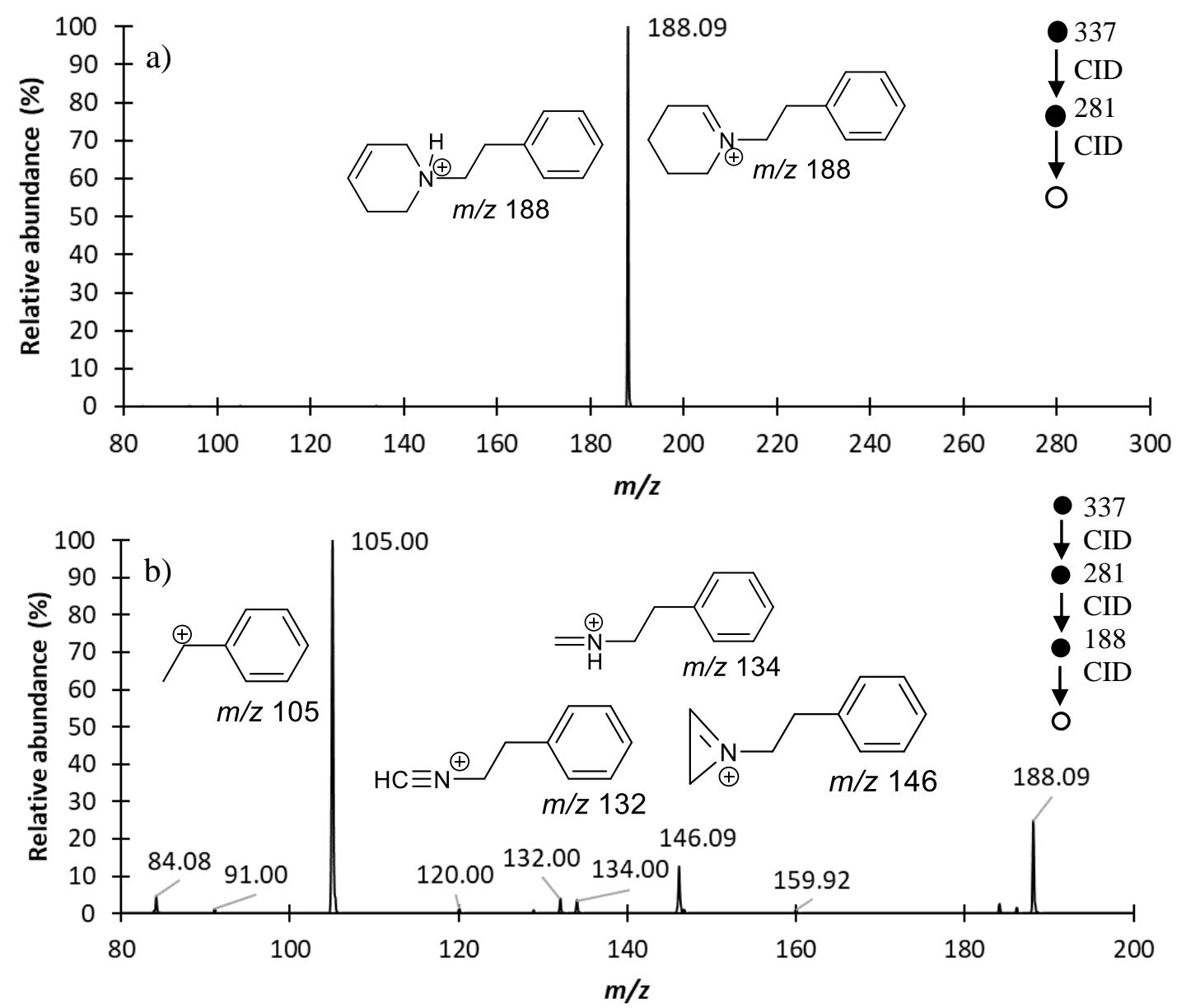

Figure 4.3. Product ion mass spectra of protonated fentanyl collected under different conditions: a) $\mathrm{MS}^{3}$ product ion spectrum for the transition $m / z 337 \rightarrow 281 \rightarrow$ at $35 \% \mathrm{NCE}$, and b) $\mathrm{MS}^{4}$ product ion spectrum for the transition $\mathrm{m} / z 337 \rightarrow 281 \rightarrow 188 \rightarrow$ at $33 \%$ NCE.

As described by Wichitnithad et al. [97], the intermediate product ion at $m / z 188$ forms through a six-centered rearrangement resulting in the loss of $N$-phenylpropanamide. However, based on our $\mathrm{MS}^{\mathrm{n}}$ studies, we have identified the intermediate product ion at $m / z, 281$ as an intermediate between the $[\mathrm{M}+\mathrm{H}]^{+}$precursor and the fragment at $\mathrm{m} / \mathrm{z}$ 188. Our proposed mechanism involves a 4-center-elimination of the methylketene from the $N$-phenylpropanamide moiety, as shown at the top of Figure 4.4. Figure 4.4 also shows the two proposed fragmentation pathways that explain the experimentally observed $\mathrm{MS}^{4}$ mass spectrum (Figure 4.3b). Pathway a) describes the formation of the product ion at $\mathrm{m} / \mathrm{z} 134$ through a retro-Diels-Alder reaction as well as the 
formation of the intermediate product ion at $m / z 160$ and ultimately, the product ion at $\mathrm{m} / \mathrm{z} 120$. Pathway b) describes the formation of the product ions at $m / z, 146,132$, and 105 from the second isobaric intermediate product ion at $m / z, 188$.

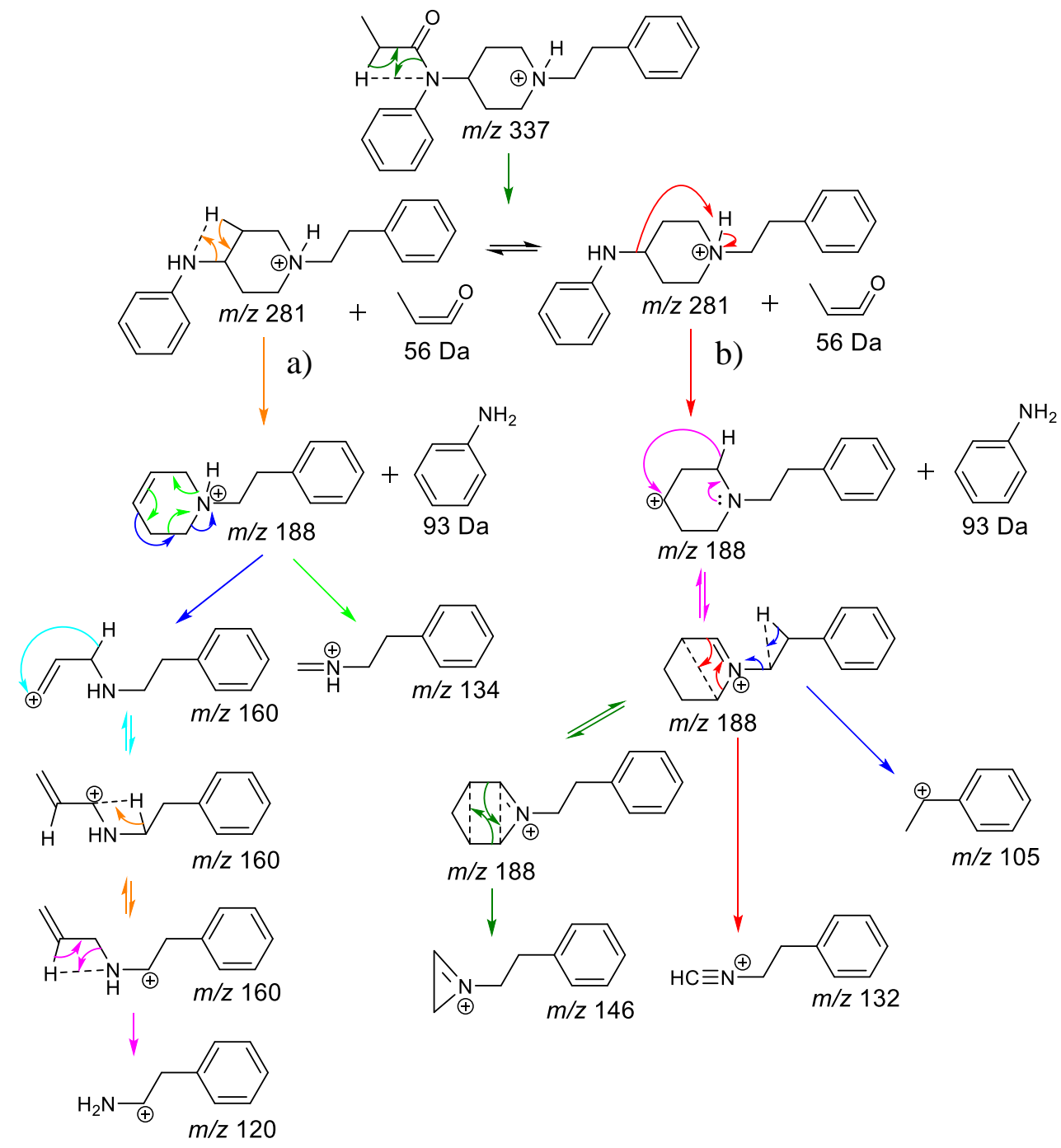

Figure 4.4. Proposed mechanisms for the formation of different product ions from two isobaric intermediates at $\mathrm{m} / \mathrm{z} 188$ [97]. Pathway a) leads to the formation of product ions at $\mathrm{m} / \mathrm{z}, 134,160$, and 120 , whereas pathway b) leads to the formation of product ions at $m / z 146,132$, and 105 .

In addition to the two isomers at $m / z, 188$ that form from the intermediate at $m / z 281$, a third isobar at the nominal mass of $\mathrm{m} / \mathrm{z} 188$ forms via fragmentation of the intermediate product ion at $\mathrm{m} / \mathrm{z} 216$ (Figure 4.5a). However, fragmentation of this particular isomer at the $\mathrm{MS}^{4}$ level only 
forms a product ion at $\mathrm{m} / \mathrm{z} 132$ (Figure 4.5b). The product ion at $\mathrm{m} / \mathrm{z}, 188$ formed through the intermediate at $\mathrm{m} / \mathrm{z} 216$ fragments differently than either of the two isomers at $\mathrm{m} / \mathrm{z} 188$ identified by Wichitnithad et al. [97].
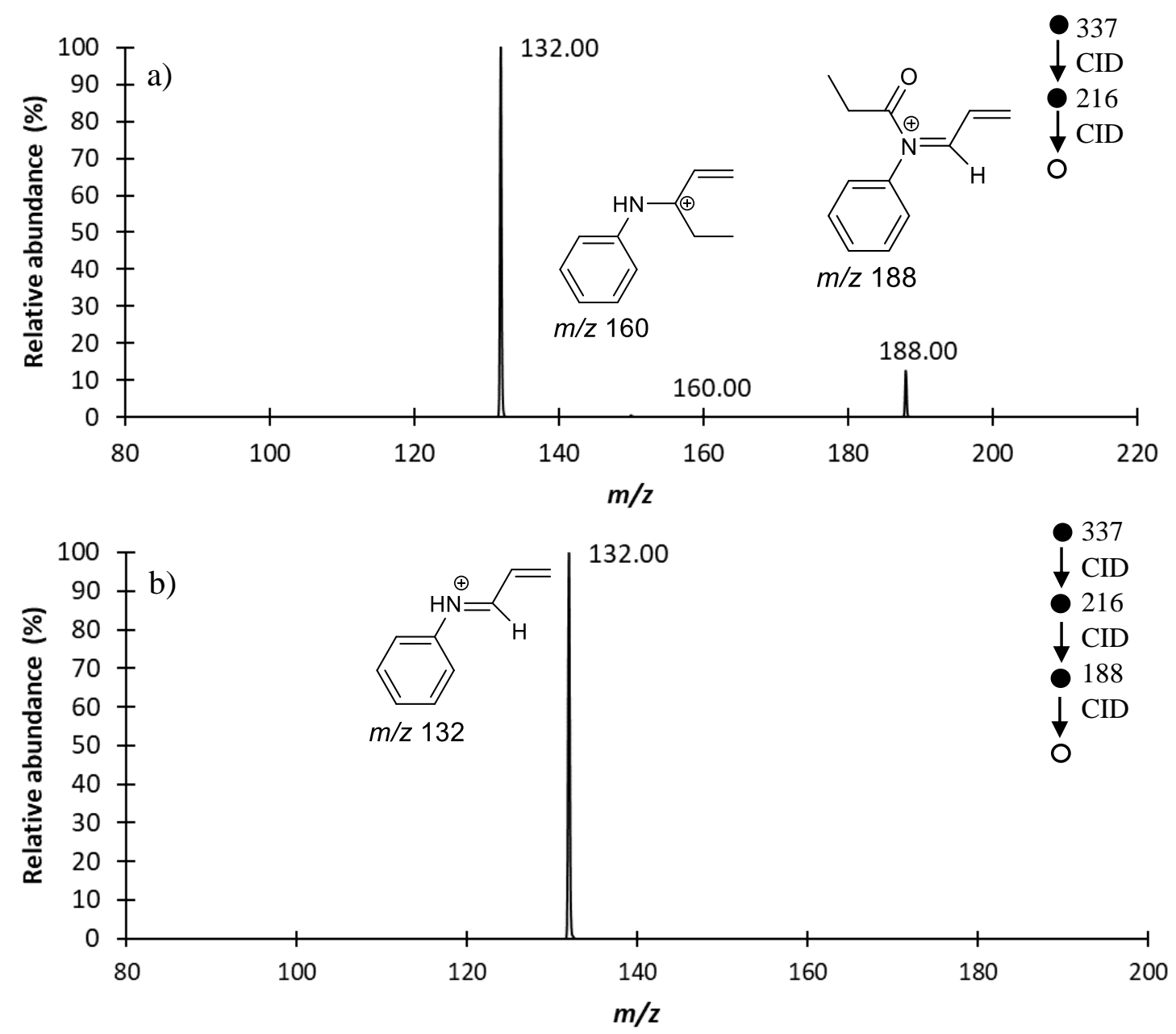

Figure 4.5. Product ion mass spectra of protonated fentanyl collected under different conditions: a) $\mathrm{MS}^{3}$ product ion spectrum for the transition $\mathrm{m} / \mathrm{z} 337 \rightarrow 216 \rightarrow$ at $35 \% \mathrm{NCE}$, and b) $\mathrm{MS}^{4}$ product ion spectrum for the transition $\mathrm{m} / \mathrm{z}, 337 \rightarrow 216 \rightarrow 188 \rightarrow$ at $25 \% \mathrm{NCE}$.

Figure 4.6 shows product ion spectra collected in pseudo-MS ${ }^{3}$ mode on the Q-TOF instrument with proposed structures of the major fragments embedded. In these spectra, in-source CID of the protonated molecular ion generated pseudo-MS ${ }^{2}$ primary fragments, which were then isolated at the $\mathrm{MS}^{2}$ level and fragmented in the collision cell to generate pseudo-MS ${ }^{3}$ spectra. CID of the fragment at $m / z 216.1300$ gave many ions at the $\mathrm{MS}^{3}$ level, one of which has an accurate mass of 
$\mathrm{m} / \mathrm{z}$ 188.1086. The accurate mass for the new product ion is consistent with an elemental composition of $\mathrm{C}_{12} \mathrm{H}_{14} \mathrm{NO}^{+}$, which has an exact mass of $188.1075 \mathrm{Da}$ (6 ppm error). In contrast, the isomers formed via the intermediate at $\mathrm{m} / \mathrm{z} 281.2020$ have an accurate mass of $\mathrm{m} / \mathrm{z}$ 188.1465. This product is consistent with an elemental composition of $\mathrm{C}_{13} \mathrm{H}_{18} \mathrm{~N}^{+}$, which has an exact mass of 188.1439 (14 ppm error). The measured masses are different by almost $200 \mathrm{ppm}$, so there can be no confusion between the different elemental compositions of the isobars formed via the two different pathways.
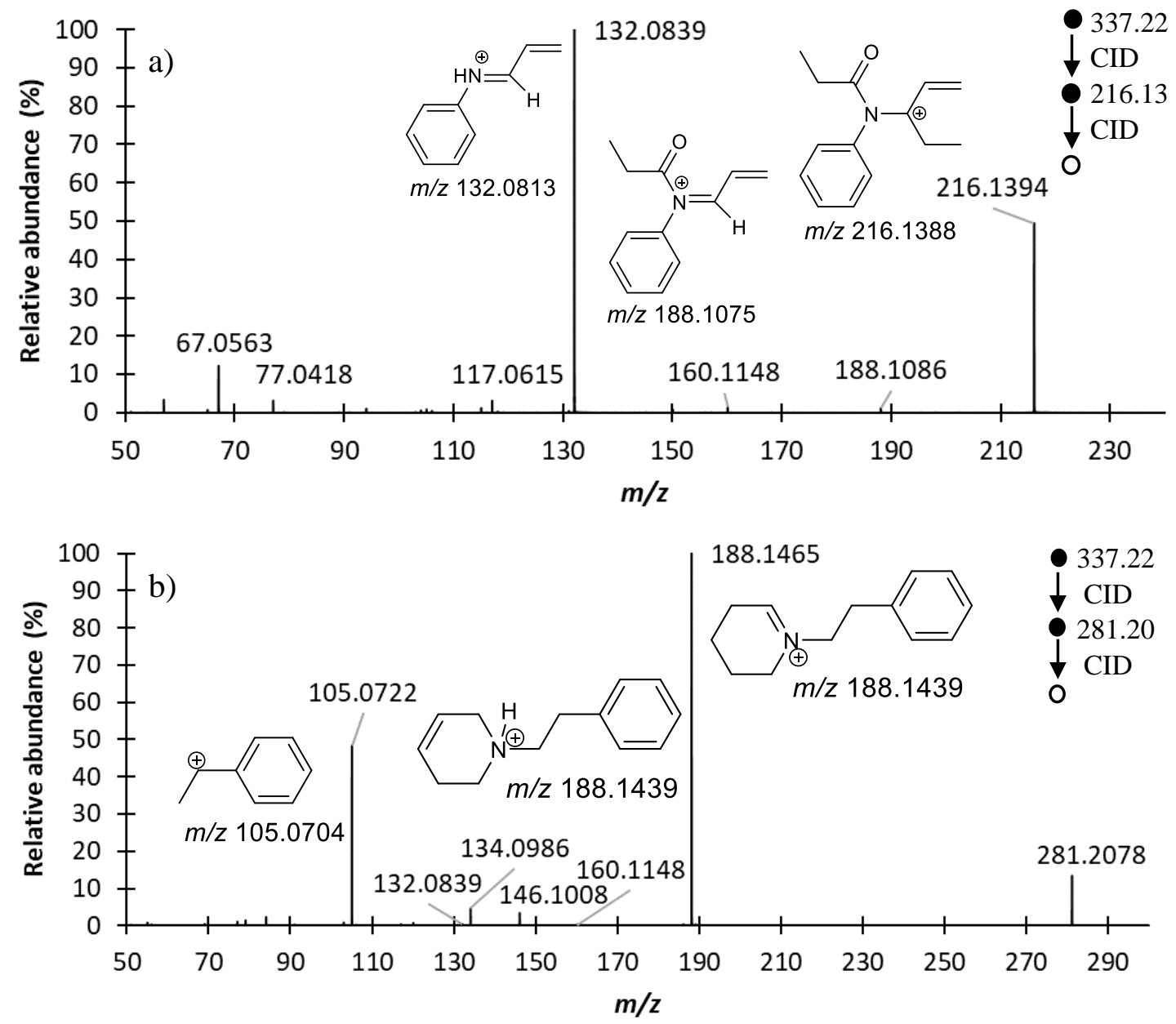

Figure 4.6. Comparison of $\mathrm{MS}^{2}$ of: a) the in-source $\mathrm{CID}$ generated intermediate product ion at $\mathrm{m} / \mathrm{z} 216.1300(25 \mathrm{eV})$ and b) the in-source CID generated intermediate product ion at $\mathrm{m} / \mathrm{z}$ $281.2000(25 \mathrm{eV})$ for fentanyl. 
As shown in Figure 4.7, the intermediate product ion at $m / z, 216$ most likely forms through the opening of the piperidine ring and charge stabilization on a tertiary carbocation as described by Thevis et al. [94]. From the structure at $m / z$ 216, the intermediate product ion at $m / z, 188$ can then form through a 4-center-elimination at the carbocation. The product ion at $\mathrm{m} / \mathrm{z} 132 \mathrm{can}$ be formed via two different pathways, one through the intermediate at $\mathrm{m} / \mathrm{z} 188$ and the other through the intermediate at $\mathrm{m} / \mathrm{z}$ 160. Formation of $\mathrm{m} / \mathrm{z} 132$ from the intermediate at $\mathrm{m} / \mathrm{z} 188$ occurs through a 4-center-elimination of methylketene. Formation of $\mathrm{m} / \mathrm{z} 132 \mathrm{from} \mathrm{m} / \mathrm{z} 160$ can occur via the 4center-elimination of ethene. Either way, the resulting product at $\mathrm{m} / \mathrm{z} 132$ contains the original aniline moiety rather than the previously described phenyl moiety. This newly identified pathway provides a third alternative structure for the isobars at the nominal mass of $m / z, 188$ in the tandem mass spectra of fentanyl.

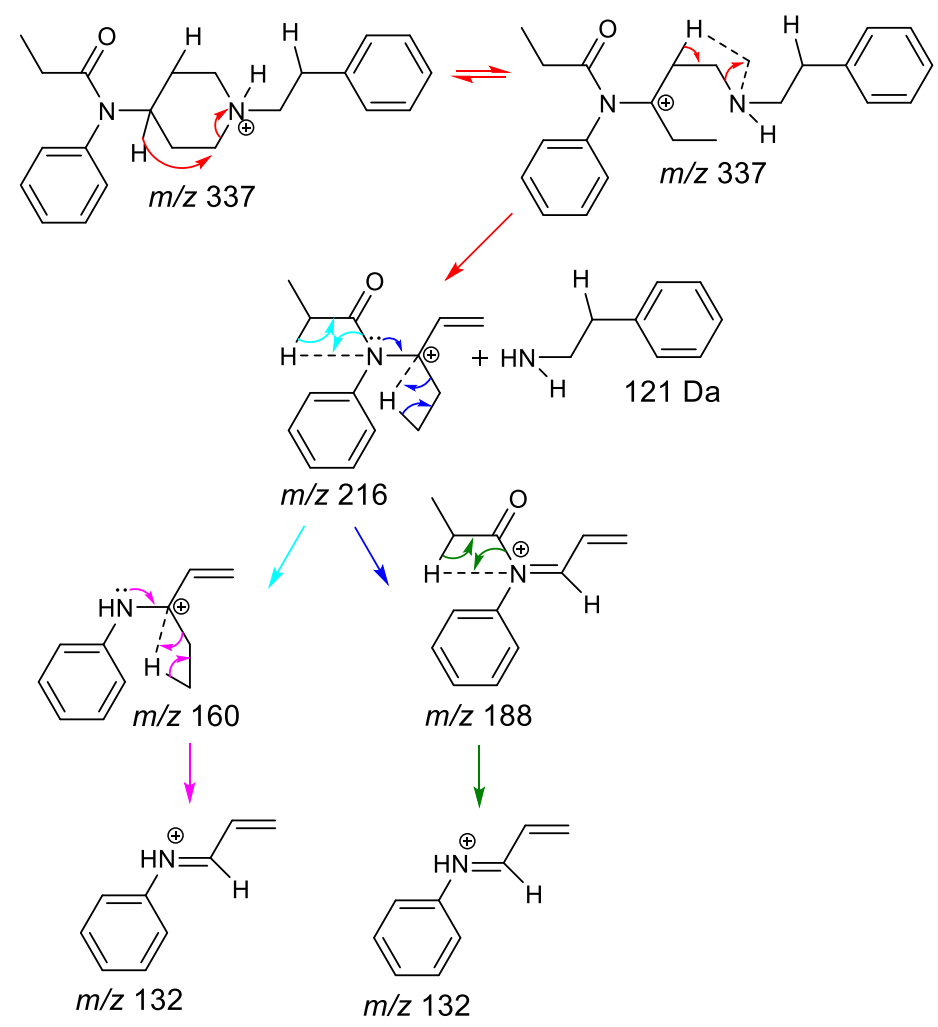

Figure 4.7. Proposed mechanisms for the formation of the novel product ion at $\mathrm{m} / \mathrm{z} 188$ from the intermediate product ion at $m / z 216$. 
$\mathrm{MS}^{\mathrm{n}}$-level analysis of protonated fentanyl also provided evidence for a fourth fragmentation pathway for a product with a nominal mass of $m / z$ 188. This fourth pathway has a measured accurate mass of 188.1465 , which is within $10 \mathrm{ppm}$ of the exact mass of $\mathrm{C}_{13} \mathrm{H}_{18} \mathrm{~N}^{+}$. This minor pathway, which occurs at approximately $0.5 \%$ of the abundance of $\mathrm{m} / \mathrm{z} 188$ in Figure 4.2, involves an R-group transfer of the propionaldehyde moiety of the molecular ion to form the intermediate product ion at $m / z$ 244. Figure 4.8a shows that although the formation of the product at $\mathrm{m} / \mathrm{z}, 190$ is the preferred product from the activation of the intermediate fragment at $m / z 244$, the product ion at $m / z 188$ is still observable at the $\mathrm{MS}^{4}$ level. Figure $\mathbf{4 . 8 b}$ shows that $\mathrm{MS}^{4}$-level fragmentation via the sequence $\mathrm{m} / \mathrm{z} 337 \rightarrow 244 \rightarrow 188 \rightarrow$ results in product ions at $\mathrm{m} / \mathrm{z} 120$ and $\mathrm{m} / \mathrm{z} 134$. Such fragmentation was not observed by Wichitnithad et al. [97], presumably because this pathway is negligible in abundance relative to the other pathways. 

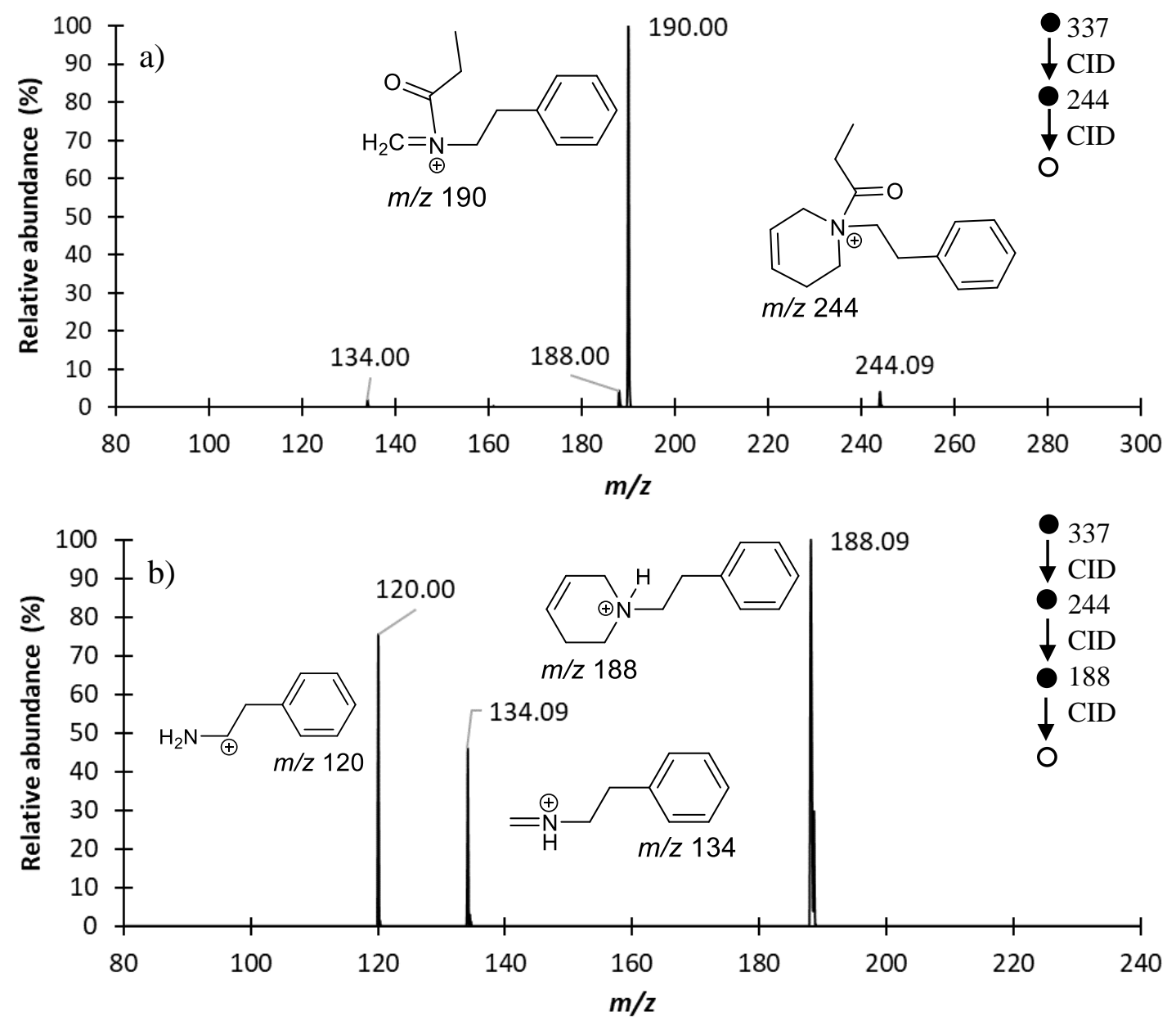

Figure 4.8. Product ion mass spectra of protonated fentanyl collected under different conditions: a) $\mathrm{MS}^{3}$ product ion spectrum for the transition $m / z 337 \rightarrow 244 \rightarrow$ at $25 \% \mathrm{NCE}$, and b) MS ${ }^{4}$ product ion spectrum for the transition $\mathrm{m} / z 337 \rightarrow 244 \rightarrow 188 \rightarrow$ at $25 \%$ NCE.

Figure 4.9 shows that the proposed R-group transfer to form the product ion at $\mathrm{m} / \mathrm{z} 244$ involves transferring the propionaldehyde group from the aniline moiety to the piperidine nitrogen via nucleophilic attack of the carbonyl carbon by the lone pair on the piperidine nitrogen atom. Transfer to the nitrogen atom is more consistent with both $\mathrm{MS}^{4}$-level spectra of fentanyl and $\mathrm{MS}^{3}$ level spectra of other fentanyl analogs. This mechanism is more easily visualized when the piperidine ring adopts a boat configuration. In the boat configuration, the propionaldehyde transfer to the nitrogen atom can occur via a sterically favored 6-center rearrangement. Transfer of the 
propionaldehyde group to the carbon atoms of the piperidine ring would involve sterically unfavorable rearrangements and weaker nucleophilic attack.

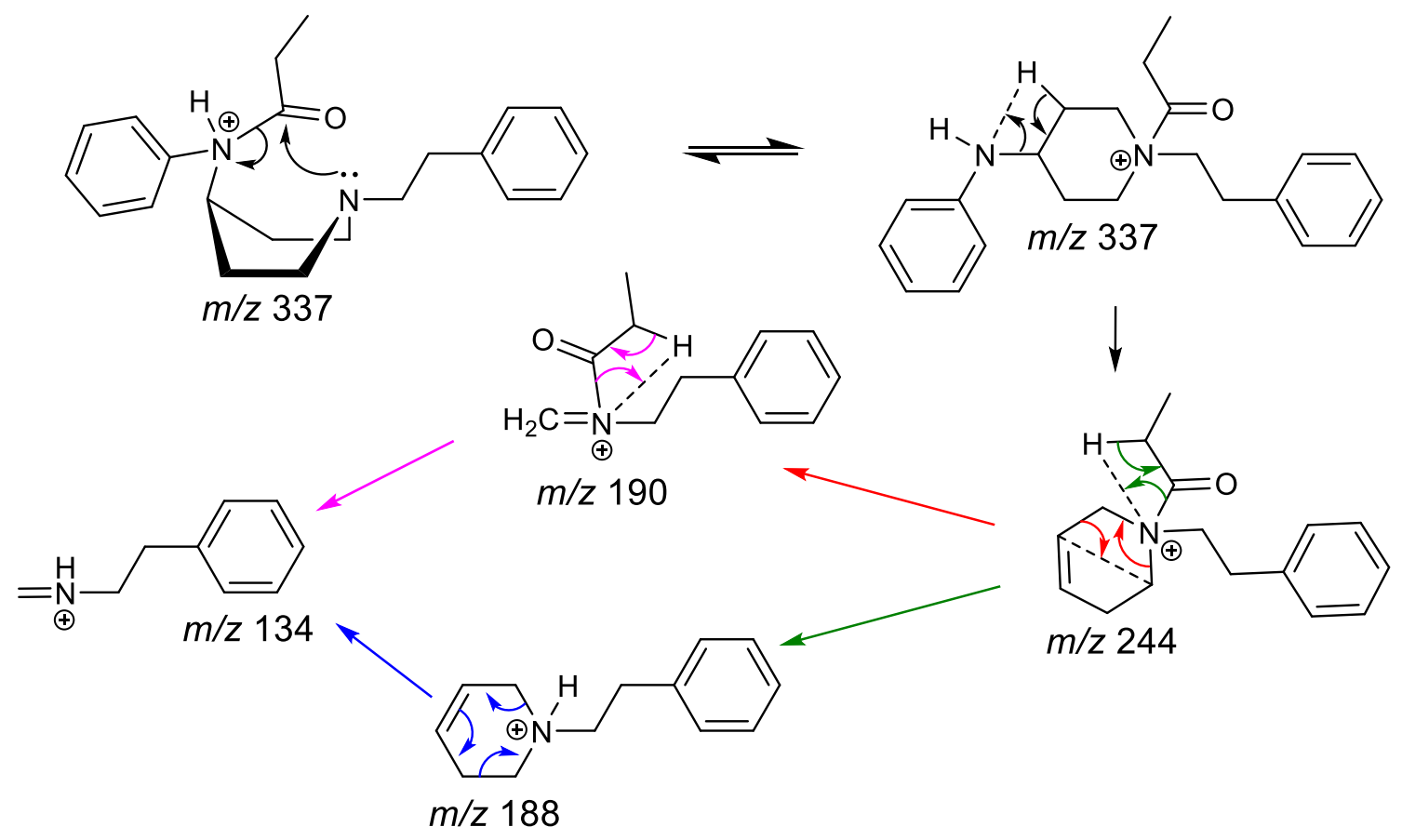

Figure 4.9. Proposed mechanisms for the formation of the product ion at $m / z, 188$ from the intermediate product ion at $\mathrm{m} / \mathrm{z} 244$.

After transfer of the propionaldehyde group, the intermediate at $m / z 244$ forms via a 4-centerelimination of aniline from the rearranged precursor. The intermediate product ion at $\mathrm{m} / \mathrm{z} 188$ forms from a 4-center elimination of methylketene, which can then undergo a retro-Diels-Alder reaction to produce the product ion at $m / z$ 134. Fragmentation at the $\mathrm{MS}^{3}$ level shows that the product ion observed at $\mathrm{m} / \mathrm{z} 134$ can also form through the intermediate product ion at $\mathrm{m} / \mathrm{z}, 190$, which is formed through the loss of cyclobutene from the intermediate product ion at $\mathrm{m} / \mathrm{z} 244$. The intermediate at $\mathrm{m} / \mathrm{z} 190$ was identified through the conserved loss of $54 \mathrm{Da}$ from a variety of fentanyl analogs [106] and the accurate mass measurements using the Q-TOF HRMS instrument (Figure 4.10). The measured accurate mass of $m / z 190.1249$ is less than $9 \mathrm{ppm}$ different than the exact mass for $\mathrm{C}_{12} \mathrm{H}_{16} \mathrm{ON}^{+}$. 


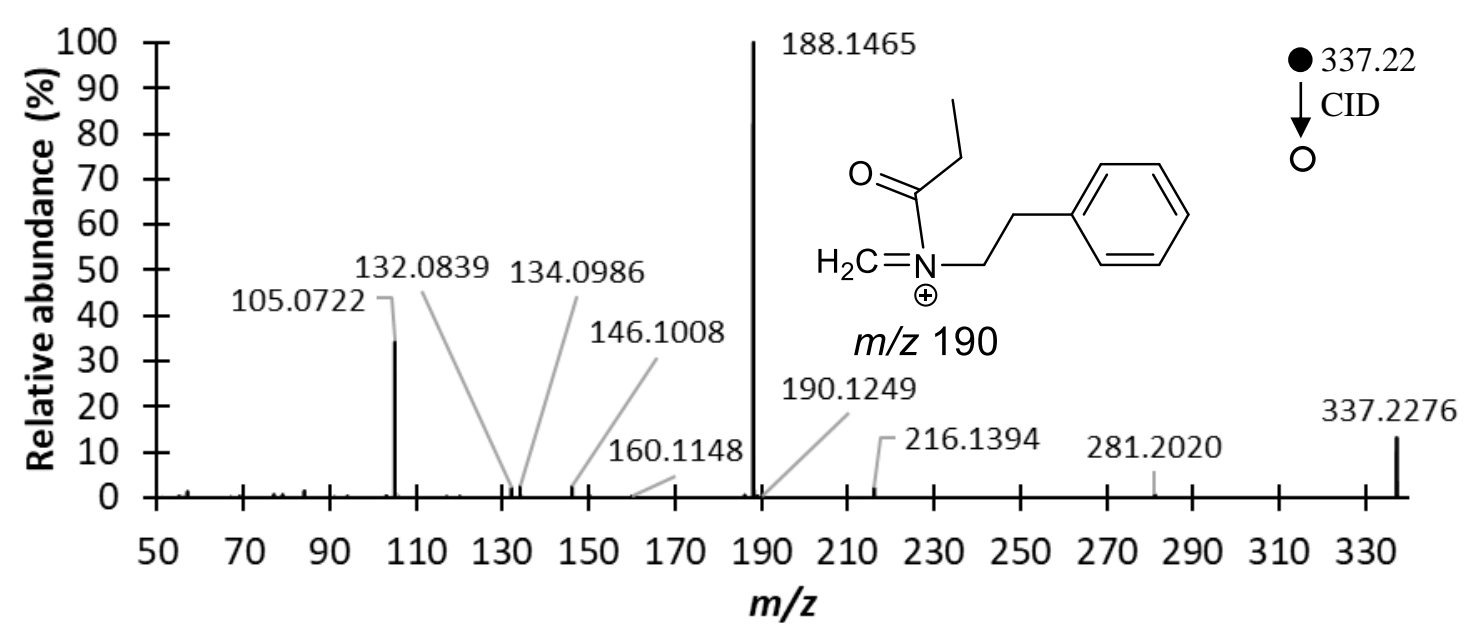

Figure 4.10. Tandem mass spectrum of fentanyl $(25 \mathrm{eV})$ and showing the product ion at $\mathrm{m} / \mathrm{z}$ 190.1249, which supports the proposed $\mathrm{C}_{12} \mathrm{H}_{16} \mathrm{ON}^{+}$elemental formula.

The analysis of the $\mathrm{d}_{5}$ version of fentanyl, which is deuterated around the aniline moiety, provides support for the three proposed mechanisms. Figure 4.11a shows the tandem mass spectrum of protonated fentanyl-d $\mathrm{d}_{5}$ observed at $\mathrm{m} / \mathrm{z} 342$ with the structures of the major fragments embedded. The incorporation of 5 deuterium atoms instead of 5 hydrogen atoms onto the aniline ring allows this moiety to be traced down each fragmentation pathway. The observed product ions at $\mathrm{m} / \mathrm{z}, 286$ and $\mathrm{m} / \mathrm{z}, 221$ confirm that the deuterated aniline moiety is present, whereas the product ion at $\mathrm{m} / \mathrm{z} 244$ indicates the deuterated aniline moiety has been lost. The proposed R-group transfer in Figure 4.9 is further supported by the observation of the product ion at $m / z 244$ from both fentanyl and fentanyl- $\mathrm{d}_{5}$. The fragment at $\mathrm{m} / \mathrm{z} 244$ occurs through the loss of $93 \mathrm{Da}$ from fentanyl and $98 \mathrm{Da}$ from fentanyl-d5. 

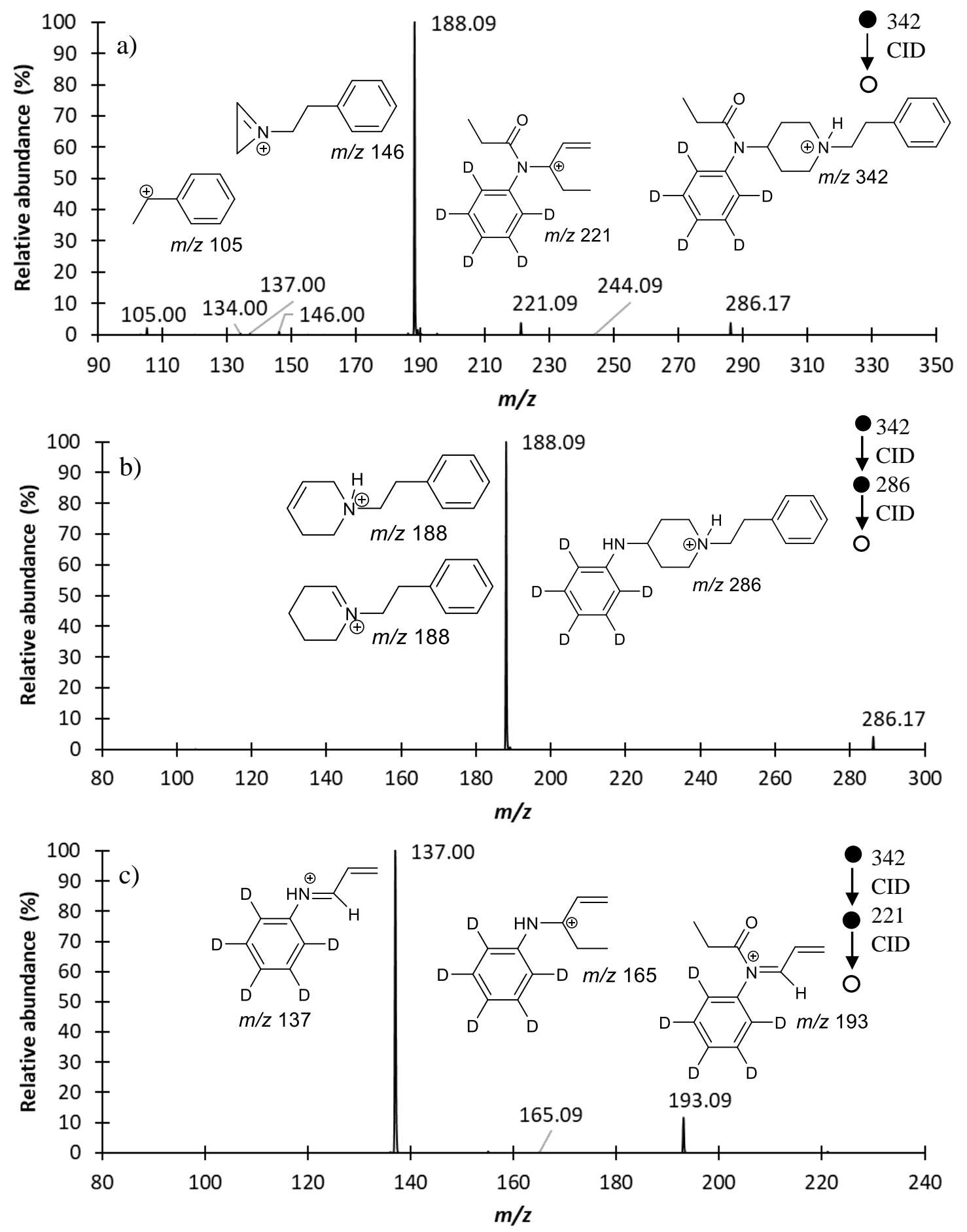

Figure 4.11. Product ion mass spectra of protonated fentanyl- $\mathrm{d}_{5}$ collected under different conditions: a) $\mathrm{MS}^{2}$ product ion spectrum of the precursor at $\mathrm{m} / \mathrm{z} 342$ at $\left.35 \% \mathrm{NCE}, \mathrm{b}\right) \mathrm{MS}^{3}$ product ion spectrum for the transition $\mathrm{m} / \mathrm{z} 337 \rightarrow 286 \rightarrow$ at $25 \% \mathrm{NCE}$, and c) $\mathrm{MS}^{3}$ product ion spectrum for the transition $m / z 337 \rightarrow 221 \rightarrow$ at $25 \%$ NCE. 
Figure 4.11b shows the $\mathrm{MS}^{3}$-level fragmentation of the intermediate at $m / z, 286$. The base peak at $m / z 188$ indicates that the deuterated aniline moiety is lost, which agrees with the proposed pathways in Figure 4.4. The most important discovery from the analysis of fentanyl- $\mathrm{d}_{5}$ is that the intermediate product at $m / z 221$ fragments into product ions at $m / z$ 193, 165, and 137 (Figure 4.11c). The product ion at $\mathrm{m} / \mathrm{z} 193$ is consistent with the deuterated version of the product ion at $m / z$ 188, which is described in Figure 4.7.

Deuterium labeling provides definitive proof that the aromatic ring that is ultimately incorporated into the product ion at $\mathrm{m} / \mathrm{z} 188$ from the intermediate product ion at $\mathrm{m} / \mathrm{z} 216$ pathway must be from the aniline moiety and not from the phenyl moiety. In contrast, the phenyl moiety is retained in the more-dominant pathways to $m / z 188$ that occur through intermediates at $m / z 281$ and $m / z$ 244. Also, observation of product ions at $\mathrm{m} / \mathrm{z} 165$ and $m / z 137$ in the deuterated fentanyl sample (Figure 4.11c) provides support for the mechanisms presented in Figure 4.7 through the incorporation of five deuterium into the proposed product ions at $\mathrm{m} / \mathrm{z} 160$ and $\mathrm{m} / \mathrm{z} 132$. Finally, Figure 4.12 contains the accurate mass measurements for the analysis of in-source CID generated $m / \mathrm{z} 221.1700$ for fentanyl-d 5 collected on the Q-TOF instrument. The incorporation of the deuterated aniline moiety is observed at every step along the proposed fragmentation pathway presented in Figure 4.7. 


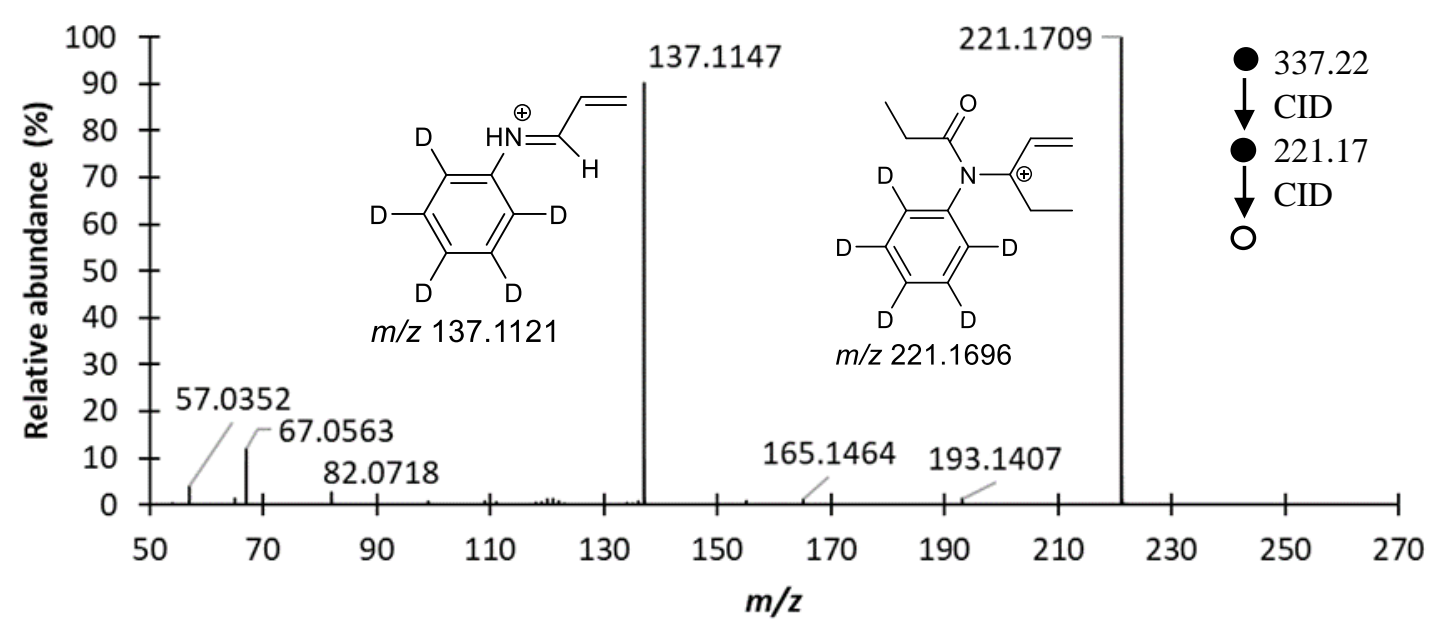

Figure 4.12. $\mathrm{MS}^{2}$ of the in-source CID generated intermediate product ion at $\mathrm{m} / \mathrm{z} 221.1700(25$ $\mathrm{eV}$ ) for fentanyl-d $\mathrm{d}_{5}$ highlighting the incorporation the deuterated aniline moiety in the product ions at $\mathrm{m} / \mathrm{z} 221.1709,193.1407,165.1464$, and 137.1147.

$\mathrm{MS}^{\mathrm{n}}$ analysis of 4-ANPP enabled the identification of similar fragmentation pathways as fentanyl. For example, Figure 4.13a shows the tandem mass spectrum of the $[\mathrm{M}+\mathrm{H}]^{+}$precursor of 4-ANPP at $m / z$ 281. The $\mathrm{MS}^{2}$ product ion spectrum shows the characteristic base peak observed at $m / z$ 188, which is present in most fentalogs. Product ions of other significance are observed at $\mathrm{m} / \mathrm{z}, 146,134$, and 105, which are all product ions of the dominant product ion at $\mathrm{m} / \mathrm{z} 188$ in Figure 4. Figure 4.13b shows that $M S^{3}$-level fragmentation of the intermediate at $m / z, 188$. The product ion distribution is identical to that of fentanyl and is consistent with the proposed mechanisms in Figure 4.4. The two new pathways to the formation of isobars at $\mathrm{m} / \mathrm{z}, 188$ for fentanyl are not possible for 4-ANPP. However, tandem mass spectrometry analysis of a variety of other fentalogs also show R-group transfers that are consistent with the mechanism shown in Figure 4.9, the results of which are the topic of ongoing work. 

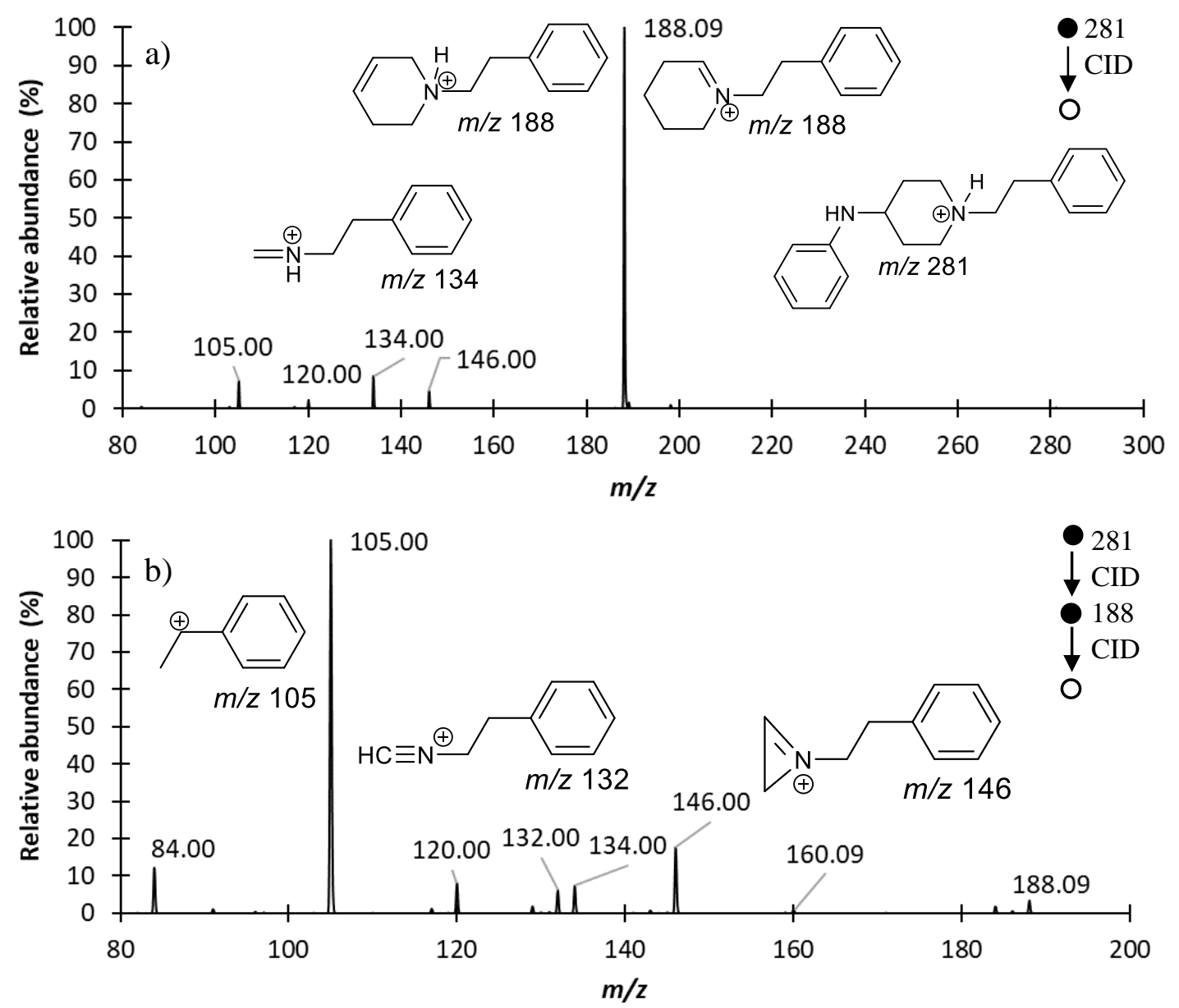

Figure 4.13. Product ion mass spectra of protonated 4-ANPP: a) $\mathrm{MS}^{2}$ product ion spectrum of protonated precursor ion at $\mathrm{m} / \mathrm{z} 281(35 \% \mathrm{NCE})$ and b) $\mathrm{MS}^{3}$ product ion spectrum for the transition $m / z 281 \rightarrow 188 \rightarrow$ at $35 \%$ NCE.

\subsection{Conclusions}

This manuscript demonstrates the use of multi-stage mass spectrometry $\left(\mathrm{MS}^{\mathrm{n}}\right)$, accurate mass measurements with HRMS and isotopic labeling for the elucidation of the fragmentation mechanisms for fentanyl and 4-ANPP. Specifically, this manuscript establishes the identification of three isobaric fentanyl product ions at $\mathrm{m} / \mathrm{z} 188$ including a novel product ion formed through the intermediate product ion at $m / z 216$. This realization has a potential impact on product ion selection for quantitative analyses. The product ion at $\mathrm{m} / z 188$ is commonly reported in literature as a product ion used for quantification based on monitoring the transition from $m / z 337 \rightarrow 188$. 
However, there are at least three isobaric fentanyl product ions at $\mathrm{m} / \mathrm{z} 188$ which have different rates and energies of formation. These differences can lead to variation in ion abundances, which can affect the accuracy and precision of quantitative analyses [97], but recognition of these different pathways can also help identify similar mechanisms in emerging fentanyl analogs. As new fentanyl analogs enter the drug market our ability to identify characteristic fragmentation pathways and conserved fragmentation mechanisms can assist medical examiners, toxicologists and seized drug analysts with the identification of novel fentanyl related compounds. 


\section{Chapter 5: The influence of chemical modifications on the fragmentation behavior of fentanyl and fentanyl-related compounds in electrospray ionization tandem mass spectrometry}

Reproduced in part with permission from J.T. Davidson, Z.J. Sasiene, G.P. Jackson, Drug Testing and Analysis, DOI: 10.1002/dta.2794.

\subsection{Introduction}

Fentanyl is a synthetic opioid that was first synthesized in 1960 by Paul Janssen [ $\underline{85}$, 107]. Due to its rapid onset and potency, fentanyl became a popular general anesthetic and was approved by the FDA in 1972 under the brand name Sublimaze [108]. Fentanyl citrate (Sublimaze) was an intravenous anesthetic that was only available to clinicians and surgeons. However, in the 1990s the introduction of transdermal fentanyl patches resulted in reports of misuse [107, 109]. In 1994, the FDA issued a warning about the dangers associated with fentanyl patches and the overprescription of potent opioids [85, 107]. Unfortunately, fentanyl also began entering the drug market through clandestine laboratories and online suppliers who synthesized new analogs faster than could be controlled by the Drug Enforcement Administration (DEA) [107]. In response, the DEA temporarily scheduled all non-classified FRCs as Schedule I narcotics to alleviate administrative and regulatory issues with prosecution [110]. The two main routes of clandestine synthesis are the Janssen and Siegfried methods, with the Siegfried method—or a modified version thereof-being the most common method for clandestine synthesis [84].

Very few fentanyl or FRC deaths were reported before 2013, and any reports were typically associated with heroin users. However, since 2013 an opioid epidemic has swept the United States and caused thousands of FRC deaths [107]. In the US, there was a $259 \%$ increase in fentanyl seizures between 2013 and 2014, and the age-adjusted death rate increased by $80 \%$ for synthetic 
opioids, excluding methadone [85]. According to the 2017 and 2018 National Forensic Laboratory Information System (NFLIS) reports, there was an increase of more than 22,000 fentanyl cases between 2016 and 2017 and more than 27,000 cases between 2017 and 2018 [루, 111].

Fentanyl and its synthetic precursors, such as 4-anilino- $N$-phenethylpiperidine (4-ANPP), are classified as Schedule II narcotics due to the medicinal value of fentanyl $[84, \underline{107]}$. However, fentanyl analogs such as $\alpha$-methylfentanyl, 3-methylfentanyl, acetylfentanyl, butyrylfentanyl, and $\beta$-hydroxythiofentanyl lack medicinal approval and are listed as Schedule I narcotics $[\underline{85}, \underline{107}$, 108]. Figure 5.1 shows a generic chemical structure for FRCs, which highlights regions of common substitution. Modification sites include substitution of the aniline ring $\left(\mathrm{R}_{1}\right)$, loss or modification of the amide $\left(\mathrm{R}_{2}\right)$, and substitution on the piperidine ring $\left(\mathrm{R}_{3}\right)$, alkyl chain $\left(\mathrm{R}_{4}\right)$ or the monocyclic substituent $\left(\mathrm{R}_{5}\right)$, including phenyl, thiol and tetrazole derivatives.

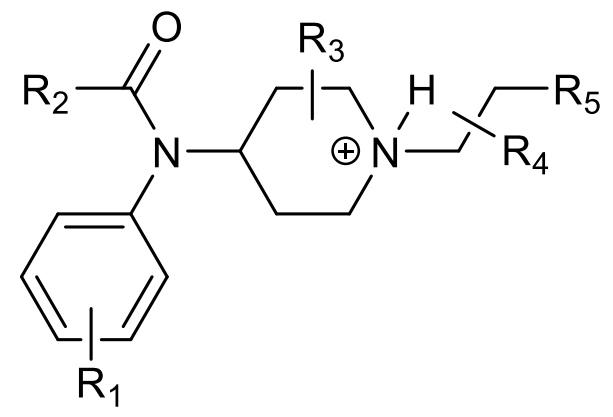

Figure 5.1. Generic chemical structure of fentanyl-related compounds (FRCs).

Because of its ubiquity, electron ionization-mass spectrometry (EI-MS) is frequently applied to the detection of FRCs, especially in combination with gas chromatography (GC). Ohta et al. analyzed 25 fentanyl derivatives and determined that 23 of the 25 compounds could be differentiated based on the combination of retention time on the GC and EI mass spectra, even in the absence of the molecular ion $\left(\mathrm{M}^{+}\right)$[112]. Kanamori et al. analyzed a series of 3-methylfentanyl 
isomers and identified the conserved nature of the fragmentation pathways with the base peak of each spectrum corresponding with the cleavage of the benzyl moiety [113].

The identification of novel FRCs has become so important that the National Institute of Standards and Technology (NIST) has developed an algorithm, known as the Hybrid Similarity Search (HSS) algorithm, that generates similarity scores based on both the fragment ions and neutral losses so that structural modifications can be identified [92]. However, the HSS algorithm still struggles with the differentiation of positional isomers, which, if not chromatographically separated, must be differentiated manually using precise comparisons of relative ion abundances. For example, Mallette et al. demonstrated the differentiation of 2-methylfentanyl and 3methylfentanyl using EI-MS, but differentiation was only possible based on the relative ion abundance of four fragment ions at $\mathrm{m} / \mathrm{z} 216,203,202$, and 160 [114]. The DEA has also conducted work with cyclopropylfentanyl and crotonylfentanyl wherein the relative ion abundance of $m / z 69$ and $m / z, 105$ was the criteria for differentiation [115]. Recently, a more novel application of EI was demonstrated through the use of a field portable nano-liquid chromatography (nLC)-EI-MS for the detection of fentanyl analogs [116].

Whereas EI-MS produces robust fragmentation that is conducive to mass spectral library searching, liquid chromatography (LC) introduction systems are also highly effective for the detection of FRCs in toxicology applications. LC introduction coupled to ESI or other ionization techniques can be used in conjunction with multiple reaction monitoring (MRM) for multiplex detection of a range of drugs and FRCs $[\underline{87}, \underline{117]}$. LC introduction can also enable multiplex detection when combined with multi-stage mass spectrometry $\left(\mathrm{MS}^{\mathrm{n}}\right)[98, \underline{99}]$ or accurate mass measurements with high-resolution mass spectrometry (HRMS) $[\underline{100}, \underline{101]}$. Another introduction method is ambient ionization, which involves the generation of gas-phase ions from untreated 
samples, reducing the need for extraction and prior chromatographic separation prior to tandem mass spectrometric analysis [118]. Specifically, the application of direct analysis in real time (DART) ionization with HRMS has shown promising results for the identification of fentanyl and FRCs $[119,120]$. Irrespective of sample introduction, tandem mass spectrometry benefits from the identification of both the molecular ion and the structural characterization through collisioninduced dissociation (CID).

The conserved fragmentation behavior of FRCs can be very beneficial for the identification of novel FRCs, if the underlying fragmentation mechanisms can be understood. Examples of mechanistic interpretation for the generation of characteristic fentanyl fragmentation include Thevis et al. [94], Wichitnithad et al. [97] and our previous work on common intermediates in the tandem MS of FRCs [121]. However, these examples are focused on either fentanyl or specific FRCs, and as such do not provide a broad, generalized approach for the identification of the location of substitutions to the core fentanyl structure.

This study investigates the effect of substitution on the fragmentation behavior of fentanyl and FRCs in ESI-MS/MS with the goal of developing a general approach for the identification of the location of substitutions to the core fentanyl structure. The 16 FRCs analyzed in this study represent a variety of permutations of substitution in Figure 1. The use of isotopic labeling, $\mathrm{MS}^{\mathrm{n}}$, and accurate mass measurements with HRMS allows the determination of the direct relationship between each product ion (MS ${ }^{n}$ ) and the elemental formula (HRMS). The use of isotopic labeling allows the labeled functionality to be followed down each fragmentation pathway as well as the identification of gas-phase rearrangements during CID. The use of both trapping and beam-type mass spectrometers also increases the applicability of these fragmentation pathways because the observations are common across different MS platforms. Finally, the ability to identify the location 
of substitution to the core fentanyl structure provides an additional tool to practitioners in the identification of emerging FRCs.

\subsection{Methods}

\subsubsection{Sample Preparation}

Thirteen FRC standards were purchased through Cayman Chemical (Ann Arbor, MI, USA), and three FRC standards were purchased through Cerilliant (Round Rock, TX, USA). The FRC standards purchased through Cayman Chemical were: 4-anilino- $N$-phenethylpiperidine (4-ANPP), fentanyl, fentanyl-d5, ortho-methylfentanyl, meta-methylfentanyl, cyclopropylfentanyl, crotonylfentanyl, para-methoxybutyrylfentanyl, methoxyacetylfentanyl, $\alpha$-methylfentanyl, $\beta$ hydroxythiolfentanyl- $\mathrm{d}_{5}$ (perdeuterated on the amide), ${ }^{13} \mathrm{C}_{6}$-para-fluorofentanyl (labeled on the phenyl moiety) and ${ }^{13} \mathrm{C}_{6}$-carfentanil (labeled on the phenyl moiety). The FRC standards purchased through Cerilliant were alfentanil, furanylfentanyl, and sufentanil-d 5 (perdeuterated on the amide). Ortho-methylfentanyl, meta-methylfentanyl, and ${ }^{13} \mathrm{C}_{6}$-para-fluorofentanyl (labeled on the phenyl moiety) are examples of FRCs with substitution at location $\mathrm{R}_{1}$ in Figure 1. Examples of FRCs with modification at location $\mathrm{R}_{2}$ of Figure 1 include: 4-ANPP (loss of propionaldehyde), cyclopropylfentanyl, crotonylfentanyl, methoxyacetylfentanyl and furanylfentanyl. ${ }^{13} \mathrm{C}_{6}{ }^{-}$ carfentanil, labeled on the phenyl moiety, is an example of a modification to location $\mathrm{R}_{3}$ in Figure 1, whereas $\alpha$-methylfentanyl is an example of a modification to location $\mathrm{R}_{4}$. Paramethoxybutyrylfentanyl, $\beta$-hydroxythiolfentanyl- $\mathrm{d}_{5}$, alfentanil, and sufentanil- $\mathrm{d}_{5}$ are examples of FRCs with a combination of modification locations, such as sufentanil-d $\mathrm{d}_{5}$ at location $\mathrm{R}_{3}$ and $\mathrm{R}_{5}$ of Figure 1. All non-deuterated standards were prepared in a solution of 49\% HPLC grade methanol (Fisher Scientific, Palo Alto, CA, USA), 49\% distilled water and 2\% acetic acid (Acros Organics, Palo Alto, CA, USA). The deuterated standards were prepared in HPLC grade methanol to reduce 
the risk of hydrogen back exchange. All solutions were prepared to a final concentration of approximately $100 \mathrm{ppm}$.

\subsubsection{Instrumentation}

\subsubsection{Thermo Scientific Velos Pro Linear Ion Trap (LIT)}

A heated-electrospray ionization source (HESI) was operated at $50{ }^{\circ} \mathrm{C}$ with a spray voltage of $4,000 \mathrm{~V}$. Nitrogen gas was used for the sheath and auxiliary gas with a flow of 8 and 5 arbitrary units, respectively. The mass spectrometer capillary temperature was $275^{\circ} \mathrm{C}$, and the scan range and normalized collision energy (NCE) were optimized for each compound and are provided with each mass spectrum. An isolation width of $1 \mathrm{Da}$ was used for all samples. Ultra-pure helium from Matheson TRIGAS (Fairmont, WV, USA) was used as the bath gas.

\subsubsection{Agilent Technologies 6538 UHD Accurate-Mass Quadrupole Time-of-Flight (Q-TOF)}

A dual ESI source was operated with a spray voltage of $3,500 \mathrm{~V}$ and a $300{ }^{\circ} \mathrm{C}$ nitrogen drying gas flow of $5 \mathrm{~L} / \mathrm{min}$ and a nebulizer flow of $30 \mathrm{psig}$ were used. The MS fragmentor and skimmer voltages, scan range, and collision energies were optimized for each compound and are labeled with each mass spectrum. An isolation width of $1.3 \mathrm{Da}$ was used for all samples. Ultra-pure nitrogen was used for the collision gas purchased through Matheson TRIGAS (Fairmont, WV, USA).

\subsubsection{Data Analysis}

Xcalibur 2.0.0.48 software and MassHunter Qualitative Analysis B.05.00 were used for the Velos Pro and Agilent data analysis, respectively. Microsoft Excel version 14 (Microsoft, Redmond, WA, USA) and ChemDraw 16.0 (PerkinElmer, Waltham, MA, USA) were used for mass spectral plots and mass spectral fragmentation pathways. 


\subsubsection{Mass Spectral Interpretation and Mechanisms}

Results from isotopic labeling, $\mathrm{MS}^{\mathrm{n}}$ and accurate mass measurements with HRMS were combined to identify characteristic fragmentation pathways of FRCs. The complex nature of gasphase mass spectral rearrangements can make it difficult to identify the exact hydrogen(s) involved in specific structural rearrangements. However, the ability to monitor specific functional groups using isotopic labeling provides deeper insight into which groups are retained and lost in a given fragmentation pathway.

\subsection{Results and Discussion}

The first phase of this project established several fragmentation pathways for protonated fentanyl and its main synthetic precursor 4-ANPP using tandem MS on a Q-TOF and a LIT [121]. The previous study confirmed the identity of three isobaric structures for the base peak at $\mathrm{m} / \mathrm{z} 188$ in $\mathrm{MS}^{2}$ spectra of fentanyl; two of the structures were previously recognized by Wichitnithad et al. [97], and the third structure has a unique elemental composition and structure but the same nominal mass of $m / z$ 188. The previous study also provided compelling evidence for an R-group transfer of the amide moiety to the $\mathrm{N}$-atom of the piperidine ring during fragmentation [121]. The current study provides additional support for this unusual mechanism and shows that the mechanism is conserved for a range of FRCs.

The combination of the LIT and HRMS instruments permit the identification of the direct relationship between intermediate product ions. On the LIT, intermediates are identified via $\mathrm{MS}^{\mathrm{n}}$ through sequential isolation and fragmentation events. The HRMS instrument allows the resolution of ions that are nominal isobars but have different exact masses. Examples are the product ions at $m / \mathrm{z} 188.1439$ for $\mathrm{C}_{13} \mathrm{H}_{18} \mathrm{~N}^{+}$and $m / \mathrm{z} 188.1075$ for $\mathrm{C}_{12} \mathrm{H}_{14} \mathrm{NO}^{+}$[97, 121], which appear at the same nominal $\mathrm{m} / \mathrm{z}, 188$ in the LIT. For the purpose of this work, primary product ions 
are defined as product ions formed directly from the precursor ion without any intermediate ion between the precursor ion and primary product ion. Secondary and tertiary product ions are the result of subsequent fragmentation events from primary product ions. The relationships identified via the LIT instrument were then applied to the HRMS data collected on the Q-TOF instrument.

\subsubsection{HESI-Velos Pro MS ${ }^{n}$}

Figure 5.2 shows the $\mathrm{MS}^{\mathrm{n}}$ fragmentation of ortho-methylfentanyl with the structures of the major fragments embedded. Isolation and fragmentation of the $[\mathrm{M}+\mathrm{H}]^{+}$precursor at $\mathrm{m} / \mathrm{z}, 351$ results in product ions at $m / z$ 295, 230, 188, 146 and 105 (Figure 5.2a). The base peak of the tandem mass spectrum is observed at $m / z 188$, which is consistent with the two isobaric product ions formed through competing mechanisms for the loss of the $N$-phenylpropanamide neutral previously demonstrated by Wichitnithad et al. [97]. The primary product ions at $\mathrm{m} / \mathrm{z} 295$ and $\mathrm{m} / \mathrm{z}, 230$ are formed through the loss of methylketene $\left(\mathrm{C}_{3} \mathrm{H}_{4} \mathrm{O}\right)$ and phenethylamine $\left(\mathrm{C}_{8} \mathrm{H}_{11} \mathrm{~N}\right)$, respectively, which are consistent with previous literature on the fragmentation of fentanyl [121].

Figure 5.2b shows the $\mathrm{MS}^{4}$ product ion spectrum for the pathway $m / z 351 \rightarrow 295 \rightarrow 188 \rightarrow$. The product ions include $m / z, 160,146,132$ and 105 are formed through the loss of ethylene $\left(\mathrm{C}_{2} \mathrm{H}_{4}\right)$, cyclopropane $\left(\mathrm{C}_{3} \mathrm{H}_{6}\right)$, cyclobutene $\left(\mathrm{C}_{4} \mathrm{H}_{8}\right)$, and tetrahydropyridine $\left(\mathrm{C}_{5} \mathrm{H}_{9} \mathrm{~N}\right)$. Isolation and fragmentation of the intermediate product ion at $\mathrm{m} / \mathrm{z} 230$ results in the formation of product ions at $m / z 202$ and $m / z$ 146, which are consistent with the methyl-substituted equivalents for fentanyl (Figure 5.2c) [121]. The methyl-substituted structures appear $14 \mathrm{Da}$ greater than the nonsubstituted analogs. 

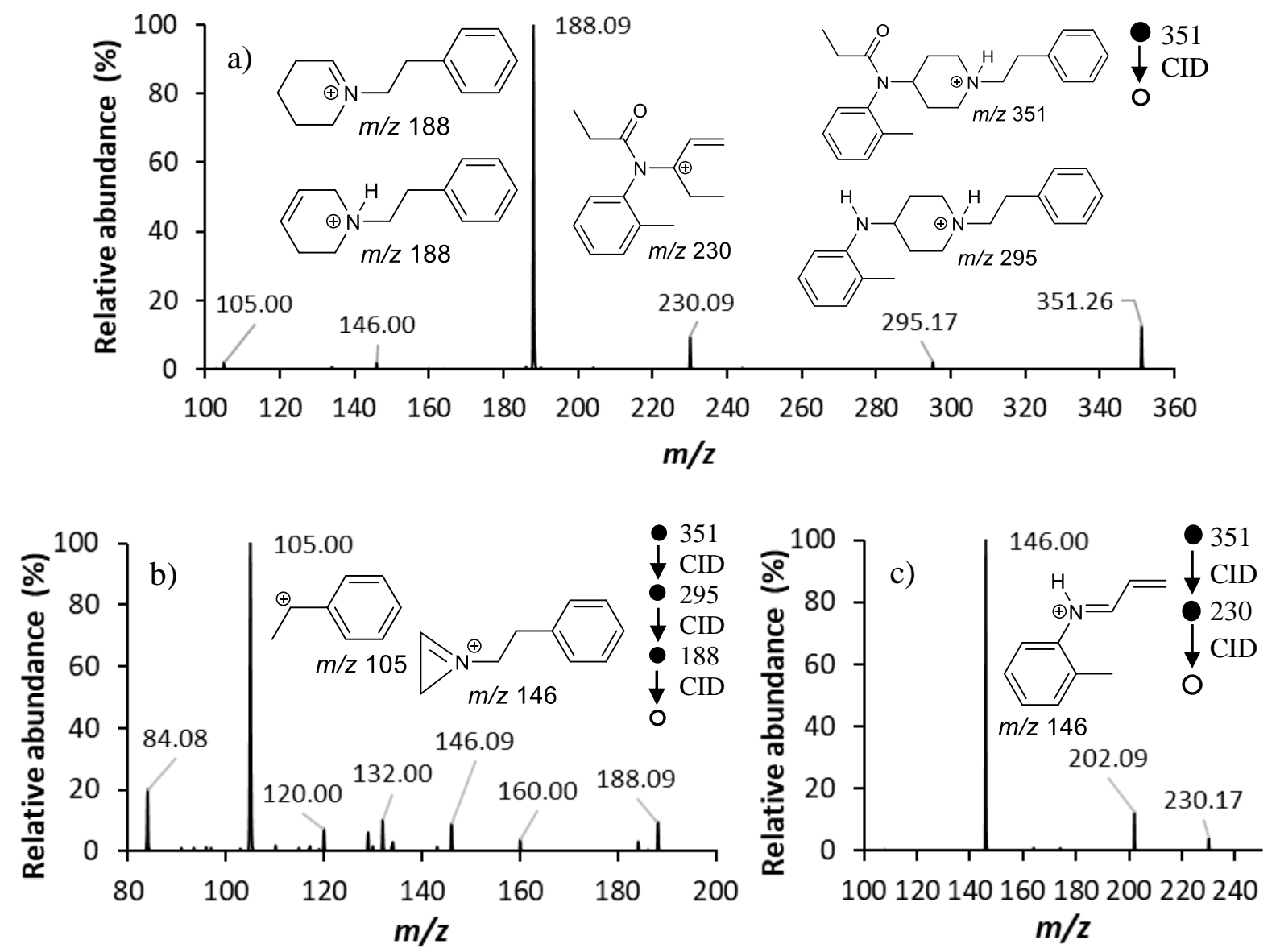

Figure 5.2. Tandem mass spectra of ortho-methylfentanyl: a) $\mathrm{MS}^{2}$ product ion spectrum of the $[\mathrm{M}+\mathrm{H}]^{+}$molecular ion (30\% NCE); b) $\mathrm{MS}^{4}$ product ion spectrum of the product ion at $\mathrm{m} / z .188$ (35\% NCE) showing the formation of product ions at $\mathrm{m} / \mathrm{z} 160,146,132$ and 105 , among others; c) $\mathrm{MS}^{3}$ product ion spectrum of the primary product ion at $\mathrm{m} / z 230$ (30\% NCE) showing the formation of $\mathrm{m} / \mathrm{z} 202$ and $\mathrm{m} / \mathrm{z} 146$.

Figure 5.3 shows the $\mathrm{MS}^{\mathrm{n}}$ fragmentation of methoxyacetylfentanyl with the major structural fragments embedded. The $\mathrm{MS}^{2}$ spectrum is dominated by the intermediate product ion at $m / z, 188$, which, like fentanyl, is comprised of at least two isobaric product ions (Figure 5.3a). The primary product ions observed at $\mathrm{m} / z 260$ and $\mathrm{m} / z 232$ correspond with the loss of aniline $\left(\mathrm{C}_{6} \mathrm{H}_{7} \mathrm{~N}\right)$ and phenethylamine $\left(\mathrm{C}_{8} \mathrm{H}_{11} \mathrm{~N}\right)$, which have been shown to be primary fragmentation pathways for fentanyl analogs $[97,121]$. It is noteworthy that the primary product ion expected at $\mathrm{m} / \mathrm{z} 281$ is not observed for this compound. Recently, Nan et al. proposed that the presence of electron-accepting 
groups on the phenylalkylamide moiety eliminated the formation of this intermediate [122]. Figure 5.3b shows the isolation and fragmentation of the primary product ion at $\mathrm{m} / \mathrm{z} 260$, which results in a dominant product ion at $\mathrm{m} / \mathrm{z} 206$. The primary product ion at $\mathrm{m} / \mathrm{z} 260$ is formed through an R-group transfer from the aniline nitrogen to the piperidine nitrogen as previously demonstrated for fentanyl [121]. The fragment at $\mathrm{m} / \mathrm{z} 206$ is formed through the loss of cyclobutene, which is observed for other FRCs wherein the novel R-group transfer is present. Finally, isolation and fragmentation of the primary product ion at $m / z 232$ results in dominant product ions at $\mathrm{m} / \mathrm{z} 204$ and $m / z 176$, which arise through the loss of ethylene $\left(\mathrm{C}_{2} \mathrm{H}_{4}\right)$ and $\mathrm{CO}$ from the $m / z 204$ intermediate product ion (Figure 5.3c). 


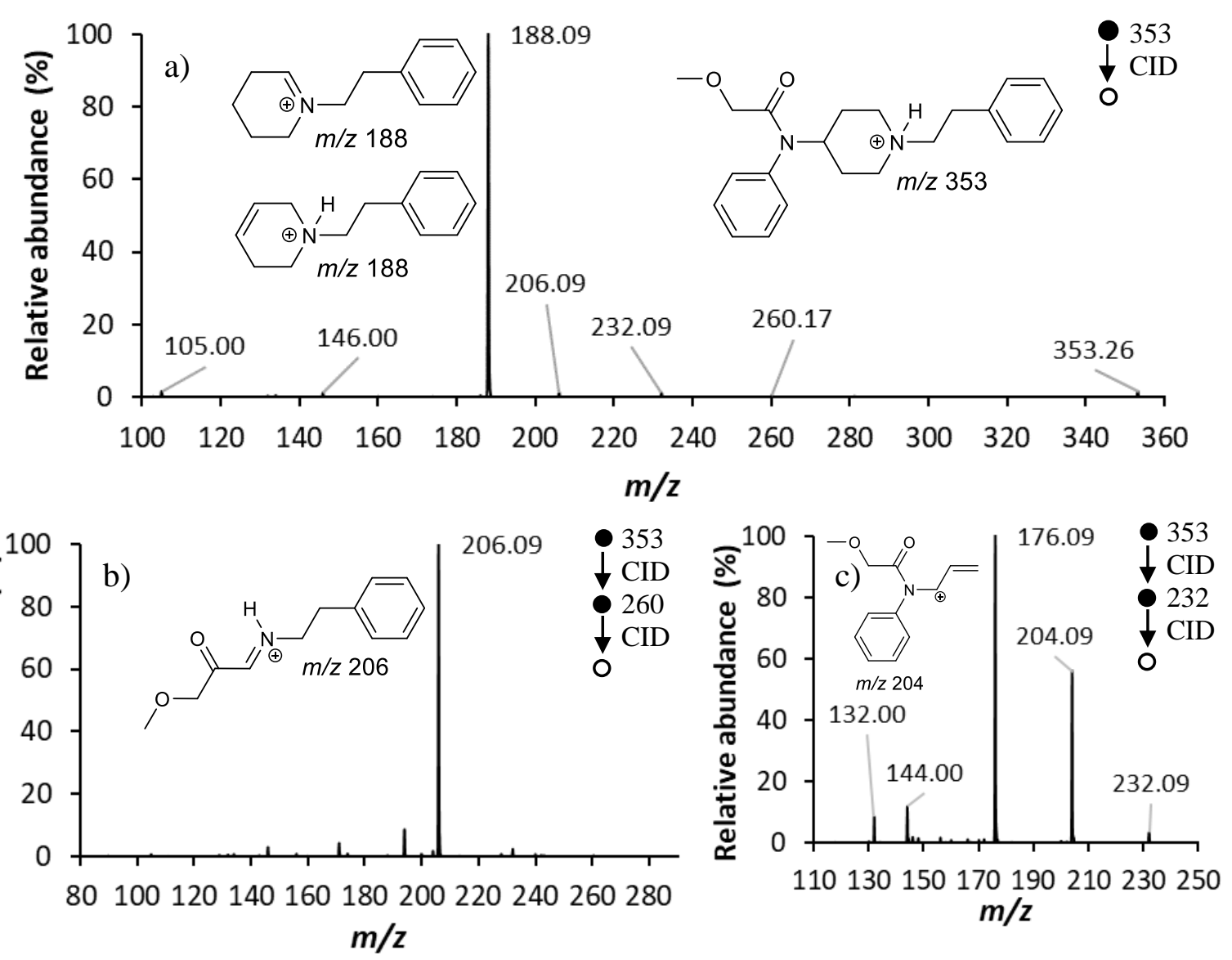

Figure 5.3. Tandem mass spectra of methoxyacetylfentanyl: a) $M S^{2}$ product ion spectrum of the $[\mathrm{M}+\mathrm{H}]^{+}$molecular ion (30\% NCE); b) $\mathrm{MS}^{3}$ product ion spectrum of the product ion at $\mathrm{m} / z 260$ (30\% NCE) showing the formation of a dominant product ion at $\mathrm{m} / z 206$; c) $\mathrm{MS}^{3}$ product ion spectrum of the primary product ion at $\mathrm{m} / z 232$ (30\% NCE) showing the formation of product ions at $m / z$ 204, 176, 144, and 132.

$\mathrm{MS}^{\mathrm{n}}$ fragmentation of sufentanil- $\mathrm{d}_{5}$ reveals an altered fragmentation pattern. Figure 5.4a demonstrates the dominance of two product ions at $\mathrm{m} / \mathrm{z} 360$ and $\mathrm{m} / \mathrm{z} 238$ in the $\mathrm{MS}^{2}$ spectrum. Formation of the product ion at $\mathrm{m} / \mathrm{z} 360$ occurs through the loss of methanol $\left(\mathrm{CH}_{4} \mathrm{O}\right)$ from the methoxymethylene substitution to the piperidine ring, which is analogous to the loss of methanol from the carboxymethylester functional group of carfentanil [95]. The base peak of the tandem mass spectrum of sufentanil- $\mathrm{d}_{5}$ is at $\mathrm{m} / \mathrm{z} 238$ and occurs through the loss of the deuterated $\mathrm{N}$ phenylpropanamide $\left(\mathrm{C}_{9} \mathrm{H}_{5} \mathrm{D}_{5} \mathrm{NO}\right)$ without the presence of any product ion through the loss of methylketene as observed for fentanyl at $m / z 281$. 

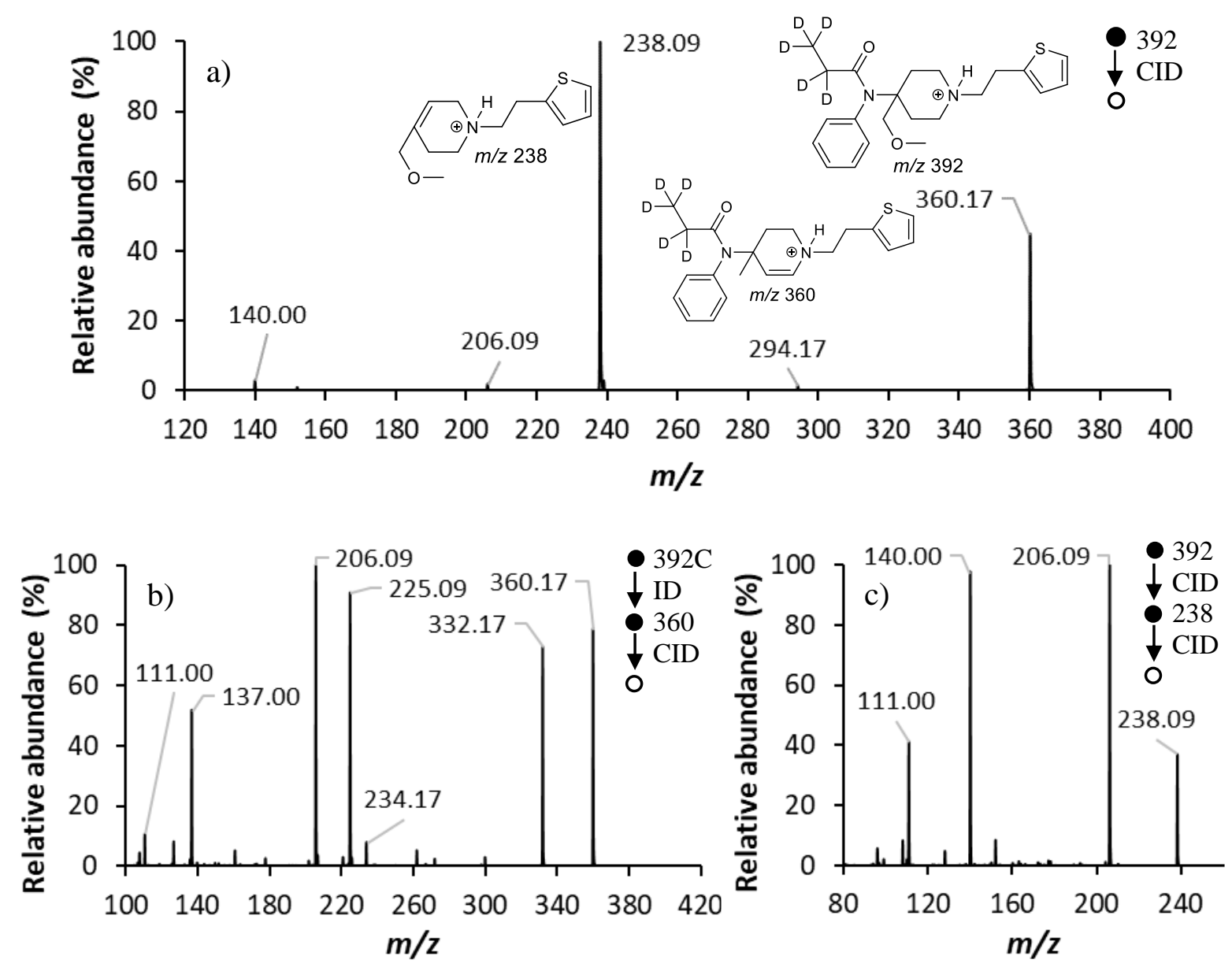

Figure 5.4. Tandem mass spectra of sufentanil- $\mathrm{d}_{5}$ : a) $\mathrm{MS}^{2}$ product ion spectrum of the $[\mathrm{M}+\mathrm{H}]^{+}$ molecular ion (30\% NCE); b) $\mathrm{MS}^{3}$ product ion spectrum of the product ion at $\mathrm{m} / z 360(30 \%$ NCE) showing the formation of product ions at $\mathrm{m} / \mathrm{z}$ 332, 234, 225, 206, 137 and 111, among others; c) MS ${ }^{3}$ product ion spectrum of the primary product ion at $m / z 238$ (30\% NCE) showing the formation of product ions at $\mathrm{m} / \mathrm{z} 206,140$ and 111.

As shown in Figure 5.4b, isolation and fragmentation of the primary product ion at $\mathrm{m} / \mathrm{z} 360$ of sufentanil-d $\mathrm{d}_{5}$ results in a large distribution of product ions including $\mathrm{m} / \mathrm{z}, 332,225,206,137$, and 111. The base peak of the $\mathrm{MS}^{3}$ spectrum for the pathway $\mathrm{m} / \mathrm{z} 392 \rightarrow 360 \rightarrow$ is observed at $\mathrm{m} / \mathrm{z} 206$ (Figure 5.4b), which occurs through the loss of the deuterated $N$-phenylpropanamide $\left(\mathrm{C}_{9} \mathrm{H}_{5} \mathrm{D}_{5} \mathrm{NO}\right)$, similar to the generation of the intermediate product ion at $\mathrm{m} / z 238$ from the $[\mathrm{M}+\mathrm{H}]^{+}$ precursor ion. Figure 5.4c is the $\mathrm{MS}^{3}$ spectrum for the pathway $\mathrm{m} / z, 392 \rightarrow 238 \rightarrow$, which produces product ions at $m / z 206,140$ and 111 with the base peak corresponding to the loss of methanol. 
Figure 5.5 shows $\mathrm{MS}^{\mathrm{n}}$ spectra of $\beta$-hydroxythiolfentanyl- $\mathrm{d}_{5}$ with the major structural fragments embedded. In the $\mathrm{MS}^{2}$ spectrum in Figure 5.5a, the primary product ion at $\mathrm{m} / \mathrm{z} 346$ dominates the spectrum and must arise through the loss of $\mathrm{H}_{2} \mathrm{O}$, which is facilitated by the presence of the hydroxyl group on the alkyl chain. The only other primary product ion observed in the $\mathrm{MS}^{2}$ spectrum is observed at $\mathrm{m} / \mathrm{z}, 250$, which forms through the loss of hydroxymethylthiol. This fragmentation behavior is different in that the loss of $\mathrm{H}_{2} \mathrm{O}$ is so dominant relative to the formation of any other primary product ions. Figure 5.5b shows the $\mathrm{MS}^{3}$ spectrum for the pathway $\mathrm{m} / z$ $364 \rightarrow 346 \rightarrow$, which results in product ions at $m / z 286,221,207,192,158$ and 147 . The base peak at $\mathrm{m} / z, 286$ of the $\mathrm{MS}^{3}$ spectrum forms through the loss of deuterated methylketene. Figure 5.5c shows the $\mathrm{MS}^{3}$ spectrum for the pathway $m / z, 364 \rightarrow 250 \rightarrow$, which results in a variety of product ions, including $\mathrm{m} / \mathrm{z} 207$ and $\mathrm{m} / \mathrm{z}$ 190, which form through piperidine ring enclosure and the loss of the deuterated methylketene moiety, respectively. 

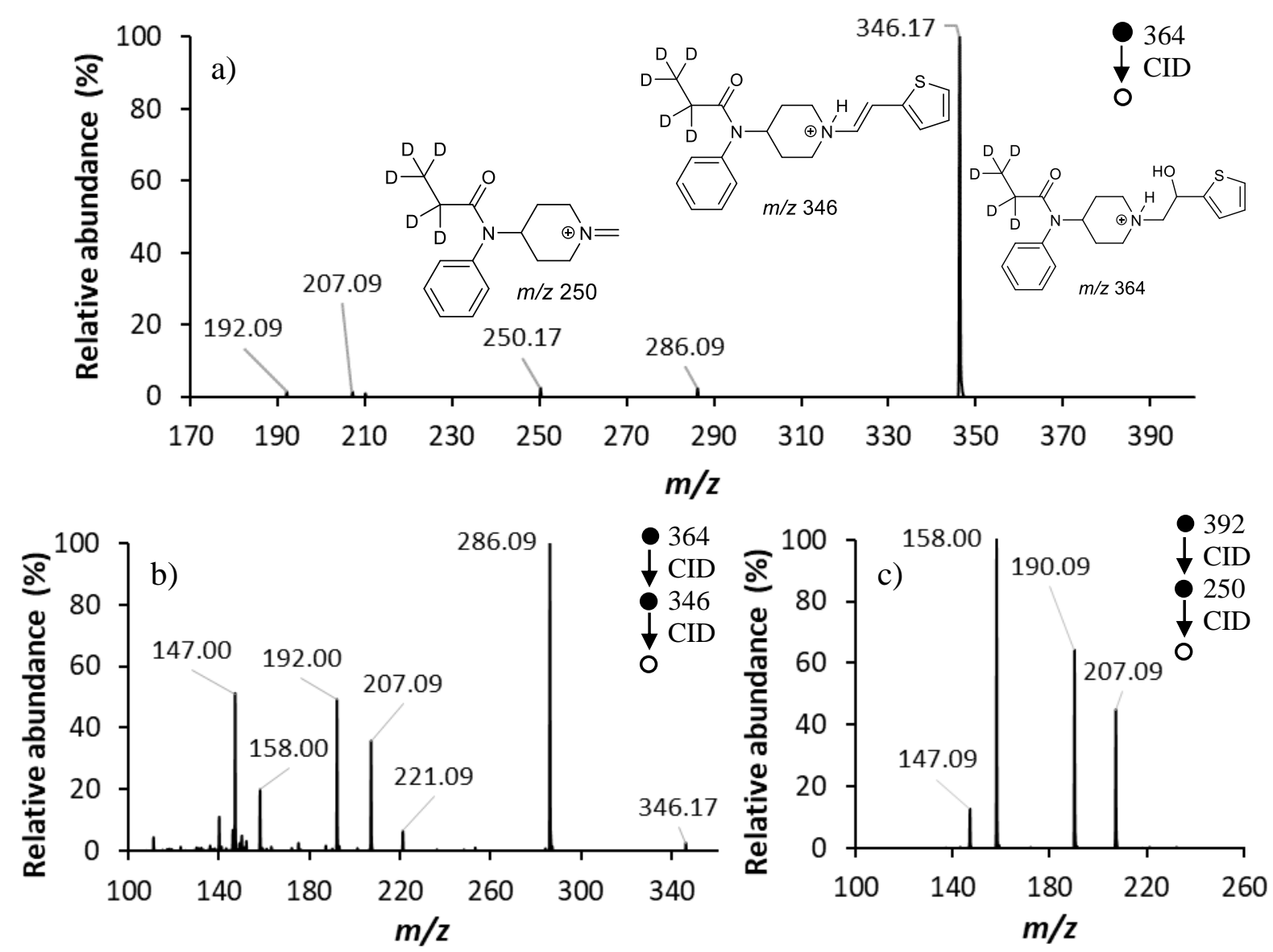

Figure 5.5. Tandem mass spectra of $\beta$-hydroxythiolfentanyl- $\mathrm{d}_{5}$ : a) $\mathrm{MS}^{2}$ product ion spectrum of the $[\mathrm{M}+\mathrm{H}]^{+}$molecular ion $(30 \% \mathrm{NCE})$; b) $\mathrm{MS}^{3}$ product ion spectrum of the product ion at $\mathrm{m} / \mathrm{z}$ 346 (30\% NCE) showing the formation of product ions at $m / z, 286,221,207,192,158$ and 147 , among others; c) $\mathrm{MS}^{3}$ product ion spectrum of the primary product ion at $\mathrm{m} / \mathrm{z}, 250$ (30\% NCE) showing the formation of product ions at $\mathrm{m} / \mathrm{z}, 207$ and $\mathrm{m} / \mathrm{z} 190$, among others.

\subsubsection{Accurate-Mass with HRMS Q-TOF}

Accurate mass measurements of the compounds in Figures 5.2-5.5 confirm the elemental formulas for the proposed structures. As an example of this capability, Figure 5.6 shows the highresolution tandem mass spectrum of para-methoxybutyrylfentanyl with the major structural fragments embedded. The primary product ions measured at $\mathrm{m} / \mathrm{z} 311.2165$ (expected at $\mathrm{m} / \mathrm{z}$ 311.2123 for $\mathrm{C}_{20} \mathrm{H}_{27} \mathrm{~N}_{2} \mathrm{O} ; 13$ ppm error) and $\mathrm{m} / \mathrm{z} 260.1663$ (expected at $\mathrm{m} / \mathrm{z} 260.1650$ for $\mathrm{C}_{16} \mathrm{H}_{22} \mathrm{NO}_{2} ; 5$ ppm error) identify the elemental formulas shown in Figure 5.6. Formation of the 
primary product ions at $\mathrm{m} / \mathrm{z} 311.2165$ and $\mathrm{m} / \mathrm{z} 260.1663$ occur through the loss of ethylketene $\left(\mathrm{C}_{4} \mathrm{H}_{6} \mathrm{O}\right)$ and phenethylamine $\left(\mathrm{C}_{8} \mathrm{H}_{11} \mathrm{~N}\right)$, respectively. The accurate mass of the base peak of this spectrum at $m / z 188.1455$ (expected at $m / z 188.1439$ for $\mathrm{C}_{13} \mathrm{H}_{18} \mathrm{~N} ; 9 \mathrm{ppm}$ error) is consistent with the structures shown in Figure 5.6. The conserved fragmentation pathways observed between the two instruments, and the consistency between the accurate mass measurements and theoretical exact masses, provides confidence that the proposed structures and pathways are typical observations in CID spectra of fentanyl analogs.

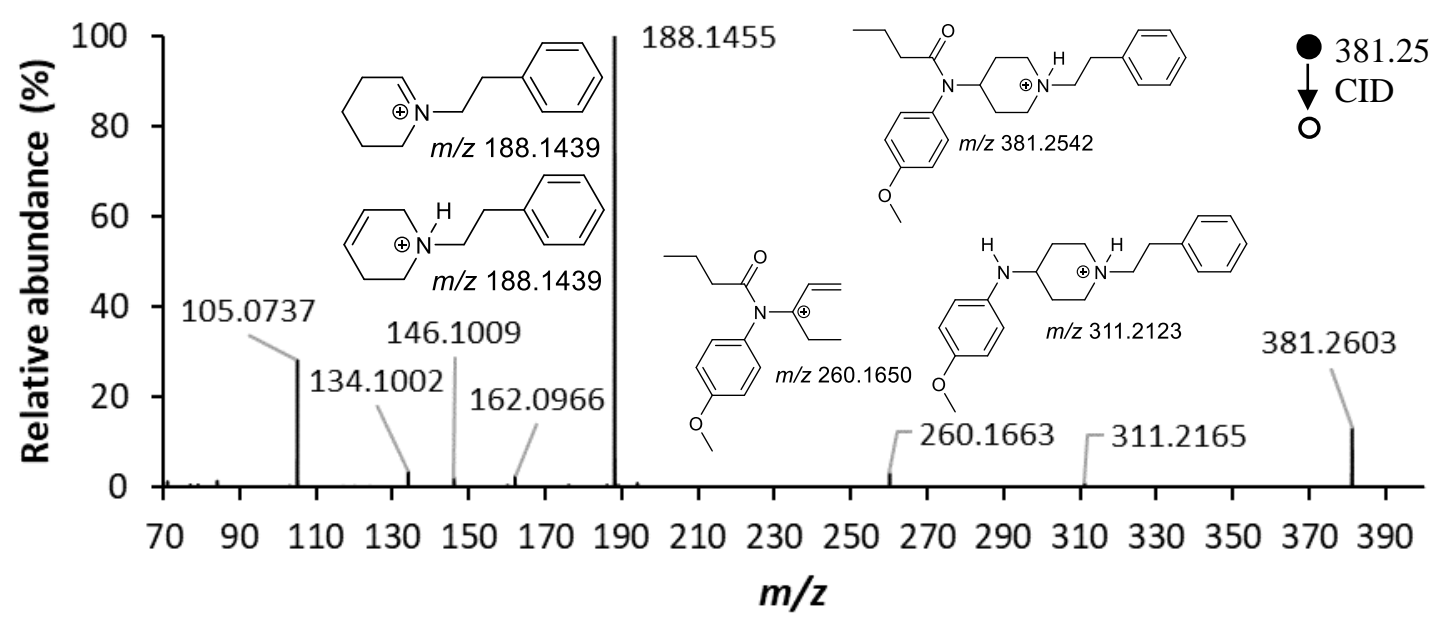

Figure 5.6. Tandem mass spectrum of para-methoxybutyrylfentanyl collected a $25 \mathrm{eV}$ collision energy, $250 \mathrm{~V}$ fragmentor voltage and $65 \mathrm{~V}$ skimmer voltage.

The HRMS tandem mass spectrum of alfentanil (Figure 5.7) highlights obvious differences in the fragmentation pathways relative to para-methoxybutyrylfentanyl (Figure 5.6) through the generation of primary product ions at $m / z 385.2381$ (expected at $m / z 385.2351$ for $\mathrm{C}_{20} \mathrm{H}_{29} \mathrm{~N}_{6} \mathrm{O}_{2} ; 7$ ppm error) and $\mathrm{m} / \mathrm{z} 268.1831$ (expected at $\mathrm{m} / \mathrm{z} 268.1773$ for $\mathrm{C}_{12} \mathrm{H}_{22} \mathrm{~N}_{5} \mathrm{O}_{2} ; 21 \mathrm{ppm}$ error). The primary product ions at $\mathrm{m} / \mathrm{z} 385.2381$ and $\mathrm{m} / \mathrm{z} 268.1831$ are formed through the loss of methanol $\left(\mathrm{CH}_{4} \mathrm{O}\right)$ and $\mathrm{N}$-phenylpropanamide $\left(\mathrm{C}_{9} \mathrm{H}_{10} \mathrm{NO}\right)$. These observations are consistent with sufentanil$\mathrm{d}_{5}$, which also contains a methoxymethylene substitution on the piperidine ring. The secondary 
product ion at $\mathrm{m} / \mathrm{z} 314.1892$ (expected at $\mathrm{m} / \mathrm{z} 314.1868$ for $\mathrm{C}_{18} \mathrm{H}_{24} \mathrm{~N}_{3} \mathrm{O}_{2} ; 8$ ppm error) forms through the loss of $\mathrm{C}_{2} \mathrm{H}_{5} \mathrm{~N}_{3}$ from the tetrazole functional group. This same $\mathrm{C}_{2} \mathrm{H}_{5} \mathrm{~N}_{3}$ loss from the tetrazole functional group also occurs from both the primary product ion at $m / z 268.1831$ and the secondary product ion at $\mathrm{m} / \mathrm{z}, 170.1061$ (expected at $\mathrm{m} / \mathrm{z} 170.1041$ for $\mathrm{C}_{6} \mathrm{H}_{12} \mathrm{~N}_{5} \mathrm{O} ; 12 \mathrm{ppm}$ error) to form product ions at $m / z, 197.1345$ (expected at $m / z, 197.1290$ for $\mathrm{C}_{10} \mathrm{H}_{17} \mathrm{~N}_{2} \mathrm{O}_{2} ; 28$ ppm error) and $m / z 99.0572$ (expected at $m / z 99.0558$ for $\mathrm{C}_{4} \mathrm{H}_{7} \mathrm{~N}_{2} \mathrm{O} ; 14$ ppm error), respectively.

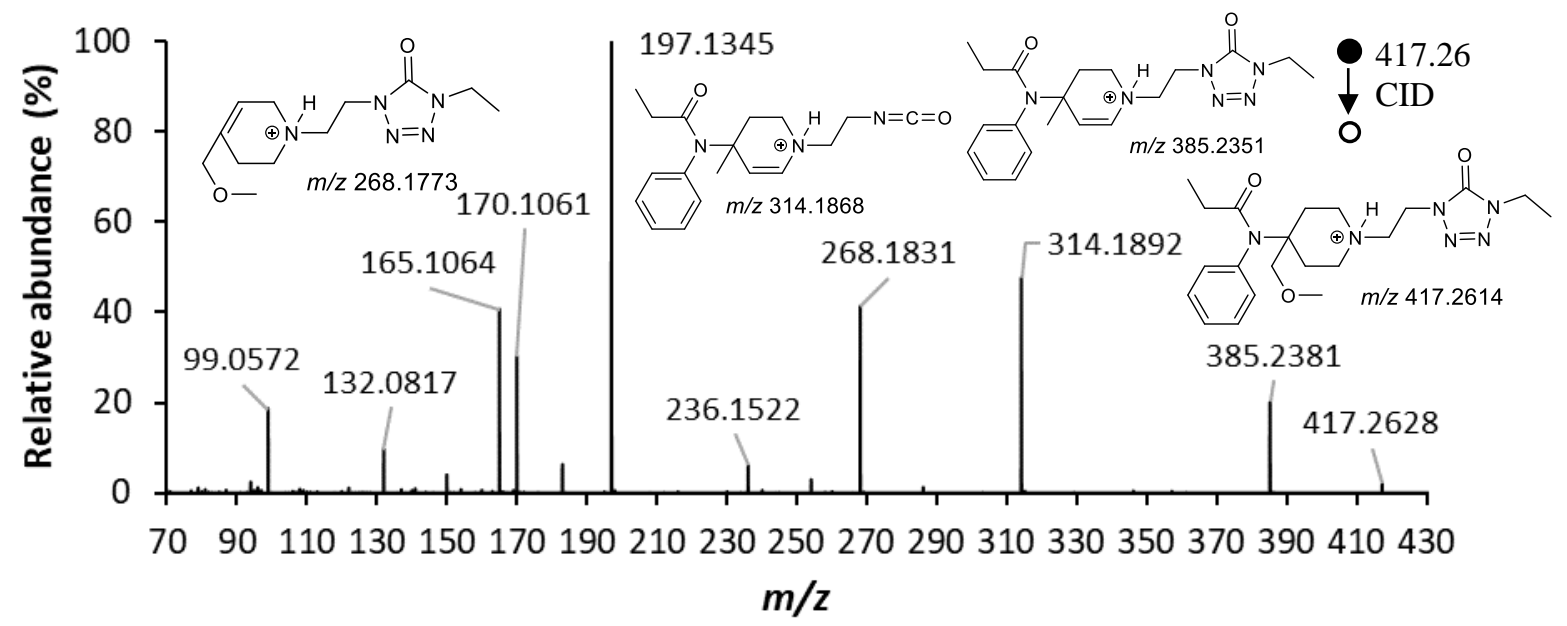

Figure 5.7. Tandem mass spectrum of alfentanil collected with a $25 \mathrm{eV}$ collision energy, $225 \mathrm{~V}$ fragmentor voltage and $65 \mathrm{~V}$ skimmer voltage.

Figure 5.8 shows the HRMS tandem mass spectrum of $\alpha$-methylfentanyl with proposed major structural fragments embedded. The base beak of this spectrum is observed at $\mathrm{m} / \mathrm{z}$ 91.0580, consistent with the elemental formula $\mathrm{C}_{7} \mathrm{H}_{7}{ }^{+}$, commonly referred to as the tropylium ion. The presence of the methyl group on the $\alpha$-carbon leads to the formation of the intermediate ion at $\mathrm{m} / \mathrm{z}$ 119.0902 (expected at $\mathrm{m} / z, 119.0860$ for $\mathrm{C}_{9} \mathrm{H}_{11} ; 35 \mathrm{ppm}$ error), which readily fragments into the tropylium ion. 


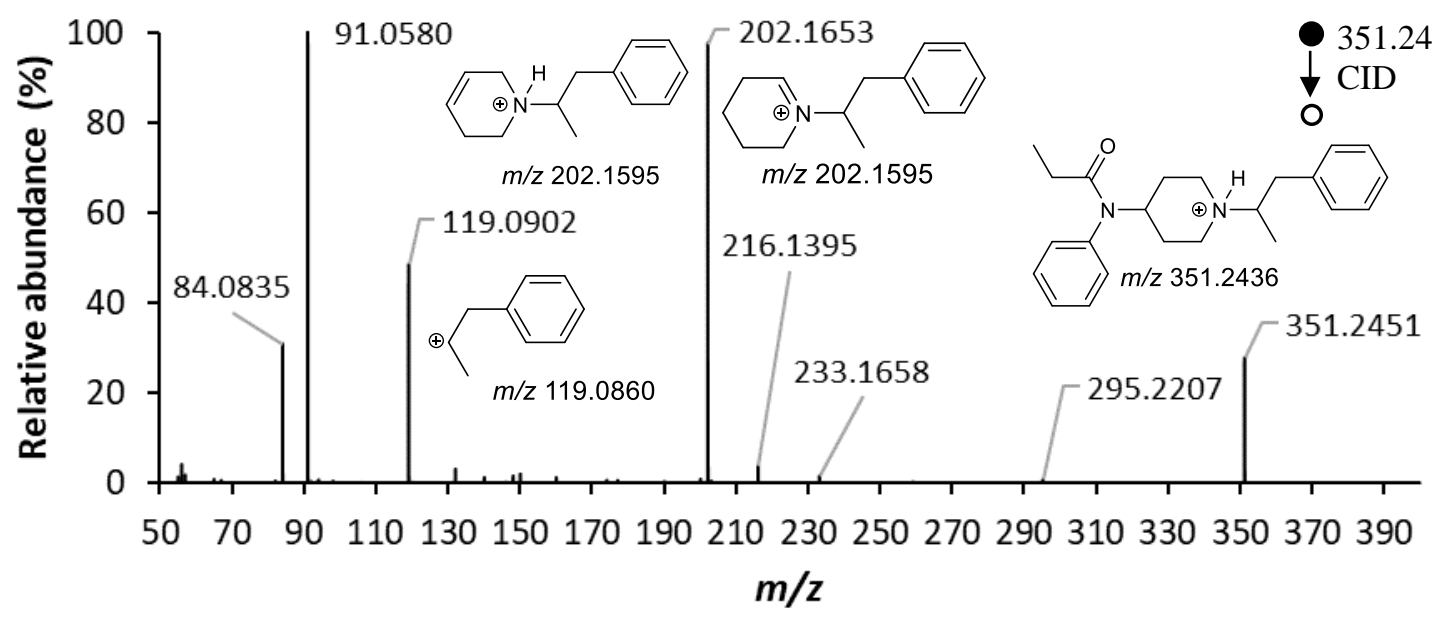

Figure 5.8. Tandem mass spectrum of $\alpha$-methylfentanyl collected with a $25 \mathrm{eV}$ collision energy, $250 \mathrm{~V}$ fragmentor voltage and $65 \mathrm{~V}$ skimmer voltage.

The other dominant product ion at $\mathrm{m} / \mathrm{z} 202.1653$ (expected at $\mathrm{m} / \mathrm{z} 202.1595$ for $\mathrm{C}_{14} \mathrm{H}_{20} \mathrm{~N} ; 29$ ppm error) is consistent with the methyl-subsituted derivative of the $\mathrm{m} / \mathrm{z} 188.1439$ base peak for non-piperidine ring substituted fentanyl analogs. The primary product ions at $\mathrm{m} / \mathrm{z} 295.2207$ (expected at $\mathrm{m} / \mathrm{z}, 295.2174$ for $\mathrm{C}_{20} \mathrm{H}_{27} \mathrm{~N}_{2} ; 11$ ppm error) and $\mathrm{m} / \mathrm{z} 216.1395$ (expected at $\mathrm{m} / \mathrm{z}$ 216.1388 for $\mathrm{C}_{14} \mathrm{H}_{18} \mathrm{NO} ; 3$ ppm error) are formed through the loss of methylketene $\left(\mathrm{C}_{3} \mathrm{H}_{4} \mathrm{O}\right)$ and 2-phenylpropylamine $\left(\mathrm{C}_{9} \mathrm{H}_{13} \mathrm{~N}\right)$, respectively. The only other primary product ion of any meaningful abundance is observed at $m / z 233.1658$ (expected at $m / z 233.1653$ for $\mathrm{C}_{14} \mathrm{H}_{21} \mathrm{~N}_{2} \mathrm{O} ; 2$ ppm error), which forms through the loss of a phenylpropyl neutral that is facilitated by the presence of the methyl group on the $\alpha$-carbon.

Figure 5.9 contains the HRMS tandem mass spectrum of ${ }^{13} \mathrm{C}_{6}$-carfentanil with the major strucutral fragments embedded. The $[\mathrm{M}+\mathrm{H}]^{+}$precursor at $\mathrm{m} / \mathrm{z} 401.2495$ (expected at $\mathrm{m} / \mathrm{z} 401.2535$ for $\mathrm{C}_{18}{ }^{13} \mathrm{C}_{6} \mathrm{H}_{31} \mathrm{~N}_{2} \mathrm{O}_{3} ; 10$ ppm error) fragments into primary product ions at $\mathrm{m} / z 369.2115$ (expected at $m / z 369.2273$ for $\mathrm{C}_{17}{ }^{13} \mathrm{C}_{6} \mathrm{H}_{27} \mathrm{~N}_{2} \mathrm{O}_{2} ; 42$ ppm error) and $\mathrm{m} / \mathrm{z} 341.2345$ (expected at $\mathrm{m} / \mathrm{z} 341.2324$ for $\mathrm{C}_{16}{ }^{13} \mathrm{C}_{6} \mathrm{H}_{27} \mathrm{~N}_{2} \mathrm{O} ; 6$ ppm error). The secondary product ion at $\mathrm{m} / \mathrm{z} 252.1754$ (expected at $\mathrm{m} / \mathrm{z}$ 252.1695 for $\mathrm{C}_{9}^{13} \mathrm{C}_{6} \mathrm{H}_{20} \mathrm{NO}_{2} ; 23$ ppm error) forms through the elimination of $\mathrm{N}$ - 
phenylpropanamide $\left(\mathrm{C}_{9} \mathrm{H}_{10} \mathrm{NO}\right)$, which ultimately forms tertiary product ions at $\mathrm{m} / z .192 .1504$ (expected at $m / z, 192.1484$ for $\mathrm{C}_{7}^{13} \mathrm{C}_{6} \mathrm{H}_{16} \mathrm{~N} ; 10$ ppm error), $\mathrm{m} / z 140.1201$ (expected at $\mathrm{m} / z 140.1171$ for $\mathrm{C}_{3}{ }^{13} \mathrm{C}_{6} \mathrm{H}_{16} \mathrm{~N} ; 21 \mathrm{ppm}$ error) and $m / z 113.0634$ (expected at $m / z, 113.0602$ for $\mathrm{C}_{6} \mathrm{H}_{9} \mathrm{O}_{2} ; 28 \mathrm{ppm}$ ). The secondary product ion at $m / z, 285.2077$ (expected at $m / z, 285.2062$ for $\mathrm{C}_{13}{ }^{13} \mathrm{C}_{6} \mathrm{H}_{23} \mathrm{~N}_{2} ; 5$ ppm error) forms through the loss of methylketene and forms tertiary product ions at $\mathrm{m} / \mathrm{z} 192.1504$ (expected at $\mathrm{m} / \mathrm{z} 192.1484$ for $\mathrm{C}_{7}{ }^{13} \mathrm{C}_{6} \mathrm{H}_{16} \mathrm{~N} ; 10 \mathrm{ppm}$ error) and $\mathrm{m} / \mathrm{z} 146.1009$ (expected at $\mathrm{m} / \mathrm{z}$ 146.0969 for $\mathrm{C}_{10} \mathrm{H}_{12} \mathrm{~N} ; 27$ ppm error).

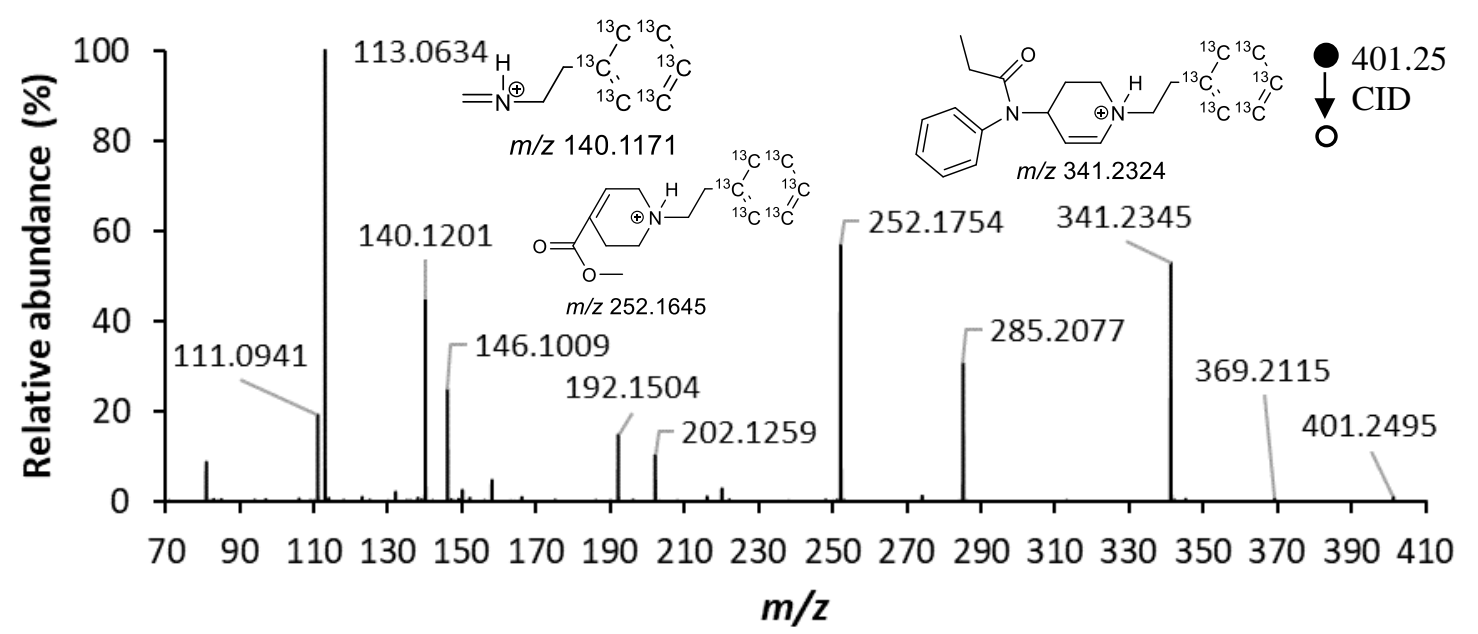

Figure 5.9. Tandem mass spectrum of ${ }^{13} \mathrm{C}_{6}$-carfentanil collected with a $25 \mathrm{eV}$ collision energy, $250 \mathrm{~V}$ fragmentor voltage and $65 \mathrm{~V}$ skimmer voltage.

Figure 5.10 shows the observed primary product ions for FRCs with ESI-MS/MS based on the use of isotopic labeling, $\mathrm{MS}^{\mathrm{n}}$, and HRMS. The eight fragmentation pathways highlight the effect of substitution to the core fentanyl structure on the observed primary product ions, with each location of substitution directing unique fragmentation pathways. Based on the FRCs analyzed, substitution to the aniline ring $\left(\mathrm{R}_{1}\right)$ and amide moiety $\left(\mathrm{R}_{2}\right)$ does not alter the fragmentation mechanisms relative to fentanyl with the lone exceptions being methoxyacetylfentanyl (Figure 5.3) and furanylfentanyl, both of which show reduced abundance for pathway 5 , likely due to the electron-accepting characteristics of the amide moiety $\left(\mathrm{R}_{2}\right)$ substitutions [122]. Pathways 6 and 7 
are still observed for methoxyacetylfentanyl and furanylfentanyl and in fact, the altered fragmentation mechanism seems to enhance to the formation of product ions through pathway 7 . In general, pathways 5, 6 and 7 are more frequently observed for FRCs with substitution to the aniline ring $\left(\mathrm{R}_{1}\right)$ and amide $\left(\mathrm{R}_{2}\right)$ moieties, which is consistent with the fragmentation behavior of fentanyl. Pathway 5 occurs through the loss of a substituted-ketene from the protonated precursor and pathway 6 arises through the opening of the piperidine ring and charge stabilization on a tertiary carbocation. Whereas pathways 5 and 6 are the dominant primary product ions observed in the protonated tandem mass spectra for $\mathrm{R}_{1}$ and $\mathrm{R}_{2}$ substituted FRCs, the product ions of pathway 7 are often observable at $\sim 1 \%$ the abundance of the base peak.

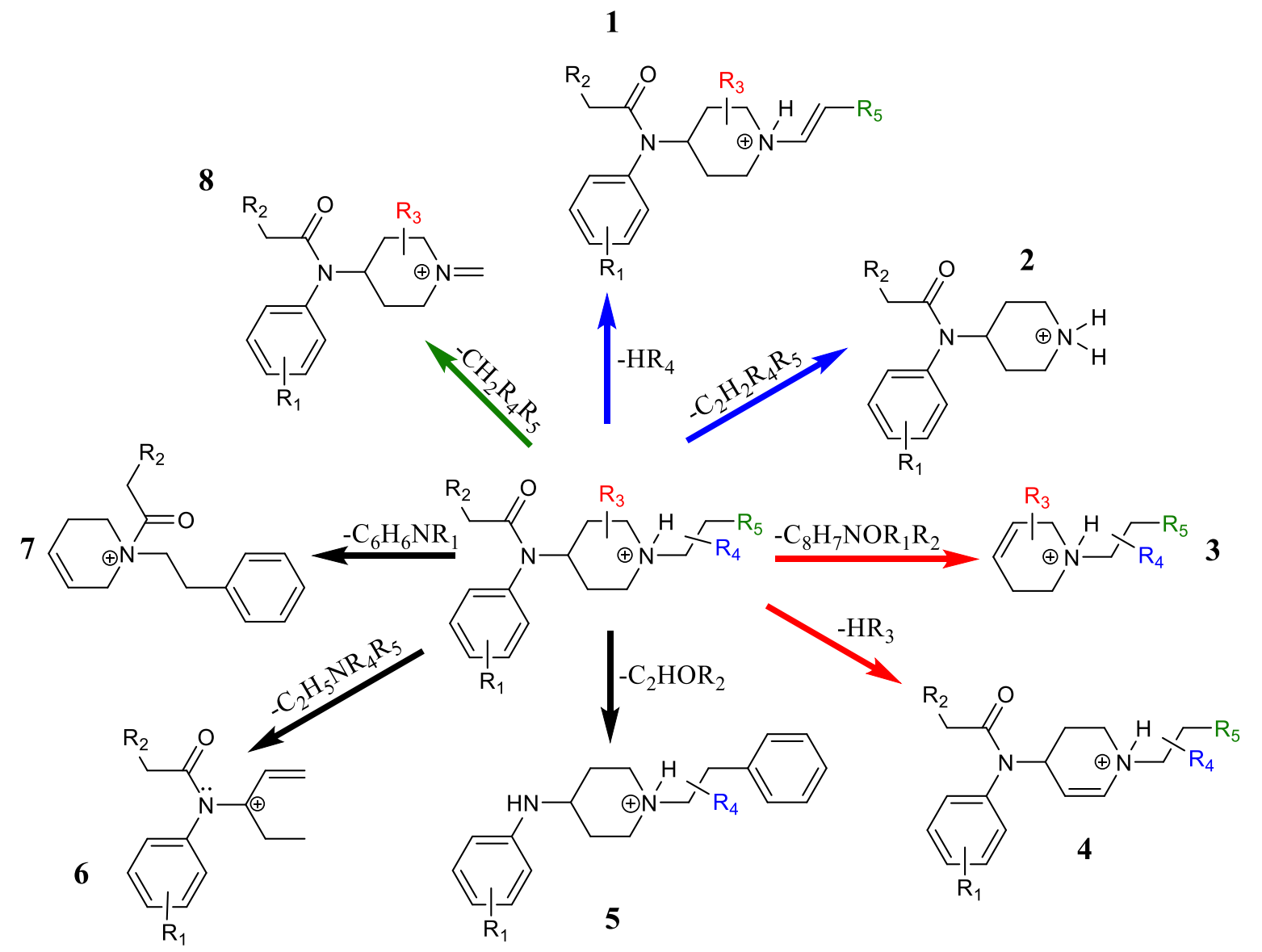

Figure 5.10. Observed primary product ions for FRCs with ESI-MS/MS. The color of an R group indicates that it tends to direct fragmentation down a pathway of the same color. 
The presence of a substituent on the piperidine ring $\left(\mathrm{R}_{3}\right)$ favors pathways 3 and 4 . Pathway 3 occurs through the direct loss of the $\mathrm{N}$-phenylpropanamide $\left(\mathrm{C}_{9} \mathrm{H}_{10} \mathrm{NO}\right)$, whereas pathway 4 arises through the loss of either a portion or the entire functional group on the piperidine ring $\left(\mathrm{R}_{3}\right)$. The piperidine ring substituted FRCs analyzed in this study were all substituted in the 4-position of the piperidine ring and as such, the fragmentation behavior described for pathway 3 is only applicable to 4-position substitutions. A recent study by Nan et al. demonstrated that piperidine ring substitutions in the 3-position do not display the characteristic behavior of piperidine ring substitutions in the 4-positions [122]. Note that pathway 3 involves the direct cleavage of the $\mathrm{N}$ phenylpropanamide moiety — with the absence of any evidence of any intermediate — through the loss of methylketene, as observed for pathway 5. Examples for pathway 3 include nominal $\mathrm{m} / \mathrm{z}$ 238 for sufentanil-d $\mathrm{d}_{5}$ (Figure 5.4), nominal $\mathrm{m} / \mathrm{z} 268$ for alfentanil (Figure 5.7), and nominal $\mathrm{m} / \mathrm{z}$ 252 for ${ }^{13} \mathrm{C}_{6}$-carfentanil (Figure 5.9).

The FRCs analyzed in this study were substituted in the 4-position of the piperidine ring with either methoxymethylene or carboxymethylester functional groups. The methoxymethylene substituted compounds favored pathway 4 through the loss of methanol as observed for product ions at nominal $\mathrm{m} / \mathrm{z}, 385$ for alfentanil (Figure 5.7) and nominal $\mathrm{m} / \mathrm{z}, 360$ for sufentanil-d $\mathrm{d}_{5}$ (Figure 5.4). In comparison, the carboxymethylester substituted compounds, such as ${ }^{13} \mathrm{C}_{6}$-carfentanil (Figure 5.9) demonstrated both the loss of methanol (i.e. $\mathrm{m} / \mathrm{z}$ 369) and the loss of methyl formate (i.e. $m / z$ 341). This fragmentation behavior may be specific to carboxymethylester compounds, which would provide an additional method of the identification of carboxymethylester substituted novel FRCs. The position of the double bond in the piperidine ring of pathway 4 is specific to the compounds analyzed in this study, and the position is likely to change depending on the position of the substituents. 
The fragmentation pathways for FRCs with substitution to the alkyl chain $\left(\mathrm{R}_{4}\right)$ are controlled by the composition of the substitution at $\mathbf{R}_{4}$. For example, pathway 1 in Figure $\mathbf{5 . 1 0}$ is the dominant fragmentation pathway for FRCs with a hydroxyl group at location $\mathbf{R}_{4}$, as seen by the peak at $m / z$ 346 for $\beta$-hydroxythiolfentanyl- $\mathrm{d}_{5}$ (Figure 5.5). In contrast, pathway 2 is favored with $\mathrm{R}_{4}$ as an aliphatic substitution on the $\alpha$-carbon, as visualized by the product ion at $m / z 233$ for $\alpha$ methylfentanyl (Figure 5.8). Despite the presence of an $\mathrm{R}_{4}$ functional group to provide additional fragmentation products to the tandem mass spectrum of a FRC, certain product ions-such as those at $\mathrm{m} / z 119$ and $\mathrm{m} / \mathrm{z} 91$, which form through secondary and tertiary fragmentation along pathway 5-are far more dominant than any of the fragments of pathway 2 , including the primary product ion at $m / z 233$ for $\alpha$-methylfentanyl in Figure 5.8. The product ions at $m / z, 119$ and $m / z, 91$ for $\alpha$ methylfentanyl (Figure 5.8) were confirmed to derive mainly through pathway 5 using $\mathrm{MS}^{3}$ of the various primary product ions for $\alpha$-methylfentanyl. In summary, the presence of aliphatic groups on the alpha carbon $\left(\mathrm{R}_{4}\right)$ enable the observation of products through pathways 1 and 2 , but these fragments are typically minor relative to the consecutive fragments of other pathways.

As demonstrated in the last example for $\alpha$-methylfentanyl, the abundance of a primary fragment ion of a pathway is not the only, or the most reliable, measure of the favorability of a fragmentation pathway. Instead, to determine the relative favorability of a pathway, we relied on $\mathrm{MS}^{3}$ spectra to determine the most abundant consecutive fragments of a pathway, and we used the sum of the product ion abundances of each spectrum to assess the relative favorability of each fragment. Therefore, although the abundance of a high mass primary product ion like $\mathrm{m} / \mathrm{z} 233$ for pathway 2 for $\alpha$-methylfentanyl might not increase much when its formation is favored by the methyl group, the low mass consecutive fragments that derive from it, such as $m / z, 84$, do show a more dramatic increase in abundance because of the additional functionality. 
Pathway 8 occurs through the loss of the substitution or a portion of the substitution to location

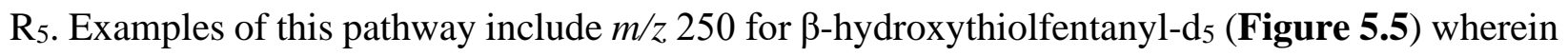
the whole $\mathrm{R}_{5}$ substitution is lost and $m / z 314$ for alfentanil (Figure 5.7), which demonstrates the loss of only a portion of the tetrazole substituent.

Table 5.1 provides a summary of the five most abundant product ions in the $\mathrm{MS}^{2}$ spectra of the $[\mathrm{M}+\mathrm{H}]^{+}$protonated precursor for the 16 FRCs analyzed in this study. The table contains both LIT data and Q-TOF data, and the peaks are ordered according to their decreasing relative abundance. Whereas the five most abundant peaks contain a great deal of overlap between the two instruments, the Q-TOF product ion spectra often contain more abundant ions with lower $\mathrm{m} / z$ values relative to the LIT data. These abundances stem from the differences in activation timescales and energies between the two instruments, and the knowledge that beam-type CID in the Q-TOF instrument provides more rapid and higher energy activation, which ultimately encourages additional consecutive fragmentation relative to the slow heating of the LIT [123-127]. 
Table 5.1. Protonated precursor ion mass-to-charge values and five most abundant product ions in decreasing order of relative abundance for each compound in this study with the LIT and QTOF instruments.

\begin{tabular}{|c|c|c|c|}
\hline Compound & {$[\mathbf{M}+\mathbf{H}]^{+}$} & $\begin{array}{l}\text { LIT product ions } \\
(\mathrm{m} / \mathrm{z}) @ 30 \% \mathrm{NCE}\end{array}$ & $\begin{array}{c}\text { Q-TOF product ions }(\mathrm{m} / \mathrm{z}) \\
@ 25 \mathrm{eV}\end{array}$ \\
\hline 4-ANPP & $m / z 281$ & $\begin{array}{c}188^{5 \#}, 134^{5 \#}, 105^{5 \#}, \\
146^{5 \#}, 120^{5 \#}\end{array}$ & $\begin{array}{c}105.07^{5 \#}, 188.14^{5 \#}, 134.09^{5 \#} \\
146.09^{5 \#}, 84.08\end{array}$ \\
\hline Fentanyl & $m / z 337$ & $\begin{array}{c}188^{5 / 6 \#}, 281^{5}, 216^{6} \\
105^{5 \#}, 146^{5 \#}\end{array}$ & $\begin{array}{c}188.14^{5 / 6 \#}, 105.07^{5 \#}, 216.13^{6} \\
134.09^{5 \#}, 146.09^{5 \#} \\
\end{array}$ \\
\hline Fentanyl- $\mathrm{d}_{5}$ & $m / z 342$ & $\begin{array}{c}188^{5 \#}, 286^{5}, 221^{6} \\
105^{5 \#}, 146^{5 \#}\end{array}$ & $\begin{array}{c}188.14^{5 \#}, 105.07^{5 \#}, 221.16^{6} \\
134.09^{5 \#}, 146.09^{5 \#}\end{array}$ \\
\hline Ortho-methylfentanyl & $m / z 351$ & $\begin{array}{c}188^{5 \#}, 230^{6}, 146^{5 / 6 \#}, \\
295^{5}, 105^{5 \#}\end{array}$ & $\begin{array}{c}188.14^{5 \#}, 105.07^{5 \#}, 146.09^{5 / 6 \#} \\
230.15^{6}, 134.09^{5 \#}\end{array}$ \\
\hline Meta-methylfentanyl & $m / z 351$ & $\begin{array}{c}188^{5 \#}, 230^{6}, 295^{5} \\
105^{5 \#}, 146^{5 / 6 \#}\end{array}$ & $\begin{array}{c}188.14^{5 \#}, 105.07^{5 \#}, 146.09^{5 / 6 \#}, \\
134.09^{5 \#}, 230.15^{6}\end{array}$ \\
\hline Cyclopropylfentanyl & $m / z 349$ & $\begin{array}{c}188^{5 \#}, 281^{5}, 228^{6} \\
105^{5 \#}, 146^{5 \#}\end{array}$ & $\begin{array}{c}188.14^{5 \#}, 105.07^{5 \#}, 69.03 \\
134.09^{5 \#}, 228.13^{6}\end{array}$ \\
\hline Crotonylfentanyl & $m / z 349$ & $\begin{array}{c}188^{5 \#}, 281^{5}, 228^{6} \\
105^{5 \#}, 146^{5 \#}\end{array}$ & $\begin{array}{c}188.14^{5 \#}, 105.07^{5 \#}, 69.03 \\
134.09^{5 \#}, 228.13^{6}\end{array}$ \\
\hline $\begin{array}{c}\text { Para- } \\
\text { methoxybutyrylfentanyl }\end{array}$ & $m / z 381$ & $\begin{array}{c}188^{5 \#}, 260^{6}, 311^{5} \\
146^{5 \#}, 134^{5 \#}\end{array}$ & $\begin{array}{c}188.14^{5 \#}, 105.07^{5 \#}, 134.09^{5 \#} \\
260.16^{6}, 162.09^{6 \#}\end{array}$ \\
\hline Methoxyacetylfentanyl & $m / z 353$ & $\begin{array}{c}188^{5}, 105^{5 \#}, 206^{7 \#} \\
232^{6}, 146^{5 \#}\end{array}$ & $\begin{array}{c}188.14^{5}, 105.07^{5 \#}, 134.09^{5 \#} \\
146.09^{5 \#}, 84.08\end{array}$ \\
\hline$\alpha$-methylfentanyl & $m / z 351$ & $\begin{array}{l}202^{5 \#}, 216^{6}, 119^{5 \#} \\
233^{2}, 295^{5}\end{array}$ & $\begin{array}{c}202.15^{5 \#}, 91.05^{5 \#}, 119.08^{5 \#}, \\
84.08,216.13^{6}\end{array}$ \\
\hline$\beta$-hydroxythiolfentanyl- $\mathrm{d}_{5}$ & $m / z 364$ & $\begin{array}{c}346^{1}, 250^{8}, 286^{1 \#}, \\
192^{1 \#}, 207^{1 \#}\end{array}$ & $\begin{array}{c}192.08^{1 \#}, 97.01,346.19^{1}, \\
147.10^{1 \#}, 111.02\end{array}$ \\
\hline${ }^{13} \mathrm{C}_{6}$-para-fluorofentanyl & $m / z 361$ & $\begin{array}{c}194^{5 \#}, 234^{6}, 305^{5} \\
111^{5 \#}, 152^{5 \#}\end{array}$ & $\begin{array}{c}194.16^{5 \#}, 111.09^{5 \#}, 140.11^{5 \#} \\
234.12^{6}, 152.11^{5 \#}\end{array}$ \\
\hline${ }^{13} \mathrm{C}_{6}$-carfentanil & $m / z 401$ & $\begin{array}{c}369^{4}, 341^{4}, 252^{5 \#} \\
220^{5 \#}, 285^{4 \#}\end{array}$ & $\begin{array}{c}113.06^{5 \#}, 252.16^{5 \#}, 341.23^{4} \\
140.11^{5 \#}, 285.20^{4 \#}\end{array}$ \\
\hline Alfentanil & $m / z 401$ & $\begin{array}{c}268^{3}, 385^{4}, 197^{3 \#}, \\
170^{3 \#}, 236^{3 \#}\end{array}$ & $\begin{array}{c}197.12^{3 \#}, 314.18^{4 \#}, 268.17^{3} \\
165.10^{3 / 4 \#}, 170.10^{3 \#}\end{array}$ \\
\hline Furanylfentanyl & $m / z 375$ & $\begin{array}{c}188^{3}, 146^{3 \#}, 254^{6}, \\
228^{7 \#}, 134^{3 \#}\end{array}$ & $\begin{array}{c}188.14^{3}, 105.07^{3 \#}, 146.09^{3 \#} \\
134.09^{3 \#}, 84.08\end{array}$ \\
\hline Sufentanil-d 5 & $m / z 392$ & $\begin{array}{c}238^{3}, 360^{4}, 140^{3 \#}, \\
206^{4 \#}, 294^{8}\end{array}$ & $\begin{array}{c}238.12^{3}, 111.02^{3 / 4 \#}, 360.21^{4} \\
140.10^{3 \#}, 206.09^{4 \#}\end{array}$ \\
\hline
\end{tabular}

*superscripts correspond with the primary product ion pathway from Scheme 1.

\# indicates secondary or tertiary fragmentation from the indicated primary product ion in Scheme 1.

Table 5.1 emphasizes the following important FRC behaviors: 1) the five most abundant product ions in the tandem mass spectra are most often either the primary product ions identified in Figure 5.10 or secondary/tertiary fragmentation thereof, 2) the five most abundant product ions 
are relatively conserved between the trapping (LIT) and beam-type (Q-TOF) mass spectrometers, and 3) If one takes into account the mass of the functional groups, the most abundant pathways and fragments are generally conserved between FRCs. For example, the base peak at $m / z, 188$ for the first 9 compounds in the table follow pathway 5 . The same pathway forms the base peak at $\mathrm{m} / z$ 202 for $\alpha$-methyl fentanyl and $m / z, 194$ for ${ }^{13} \mathrm{C}_{6}$-para-fluorofentanyl, for example. There are also several situations where product ions can be formed through two competing pathways, such as the product ion at $m / z 111$ for sufentanil-d $\mathrm{d}_{5}$ through pathways 3 and 4 . The real impact of this knowledge is that the product ions formed from ESI-MS/MS can be used to identify the mass and location of substitutions based on shifts in mass due to the additional substituent. However, we recognize that the use of this knowledge currently requires extensive manual interpretations, and that most practitioners will struggle to apply these general trends. The toxicology and seized drug communities could benefit from an automated spectral similarity search, similar to the HSS algorithm for EI spectra [92] that was applicable to tandem mass spectra of protonated FRCs. Until then, analysts will have to rely on manual interpretations following a generalized set of rulessuch as those proposed here-to identify emerging FRCs.

\subsection{Conclusions}

The combination of isotopic labeling, $\mathrm{MS}^{\mathrm{n}}$, and accurate mass measurements with HRMS was used to develop general rules for the fragmentation of fentanyl analogs and the identification of substitutions to the core fentanyl structure. A series of 16 FRCs with substitutions at five common locations to the core fentanyl structure was used to identify general fragmentation pathways and their propensity to direct fragmentation down particular pathways. The identification of primary product ions for FRCs substituted at each of the five locations of substitution as well as the relative consistency of the five most abundant product ions between the LIT and Q-TOF instruments 
provides guidance to the forensic community about how to identify the location of substitution for FRCs that is applicable across different MS platforms. Finally, the identification of the conserved fragmentation pathways, when accounting for differences in the mass and location of the substituent for FRCs, provides an additional tool for the identification of novel FRCs to toxicologists and seized drug analysts. 


\section{Chapter 6: Comparison of in-source collision-induced dissociation and beam-type collision-}

induced dissociation of synthetic cathinones and fentanyl analogs using a high-resolution quadrupole time-of-flight (Q-TOF) mass spectrometer

\subsection{Introduction}

Electron ionization mass spectrometry (EI-MS) is an invaluable tool for the structural identification of unknown organic compounds such as drugs and drug metabolites [128, 129]. In particular, the generation of mass spectral databases based on standardized ionization conditions with $70 \mathrm{eV}$ electrons permits remarkable consistency and reproducibility in the spectra collected on different instruments and by different vendors [129]. However, the EI-MS spectra of many organic compounds, such as synthetic cathinones and fentanyl analogs, do not contain abundant molecular ions, which are incredibly helpful for determining the molecular weight of an unknown $[15,91]$. An approach to solve this issue is the application of soft ionization sources, like electrospray ionization (ESI), which results in little to no fragmentation of the $[\mathrm{M}+\mathrm{H}]^{+}$pseudomolecular ions. When used in combination with tandem mass spectrometry (MS/MS), ESIMS/MS is capable of obtaining both the molecular weight information and structurally informative fragments to help identify compounds and distinguish isobaric and isomeric ions [125, 130].

Tandem mass spectrometry has traditionally involved collisional activation of an isolated precursor ion with a neutral bath gas, commonly termed collision-induced dissociation (CID) [125]. The process of CID for beam-type instruments involves 10s-100s of collisions with a neutral gas like nitrogen or argon as the precursor ion passes through the collision cell. In contrast, CID in trapping-type instruments involves 100s of collisions between the stored precursor and the neutral bath gas, which is typically helium [51, 127]. Trapping-type CID therefore tends to promote lower energy pathways relative to beam-type CID [5]. However, not all instruments 
include a collision cell or ion trap, so in-source CID has evolved as a way to accomplish CID in the absence of tandem-MS capabilities. In-source CID is achieved by manipulating the acceleration voltages as ions transition from the atmospheric pressure ionization source to the high vacuum of the mass analyzer. Manipulation of these voltages causes ions to undergo energetic collisions with residual background gases and, ultimately, fragment. Collision conditions for insource CID are usually at energies up to hundreds of $\mathrm{eV}$ and at pressures on the order of 1 mbar [127].

In-source CID has proven to be successful with the fragmentation of a variety of macromolecules, including multiply charged peptides [131, 132], porphyrins [133] and cytochrome c [134] and smaller molecules such as opiates [135], synthetic cathinones [136] and fentanyl analogs [119]. In-source CID has many monikers, including nozzle/skimmer activation [131, 134], ESI-CID, up-front CID, and transport-region CID [129]. Such is the prominence and reliability of in-source CID that mass spectral libraries now exist to assist with the identification of unknowns [137]. The in-source CID libraries usually contain spectra at three different acceleration voltages or at an average of one high and one low acceleration voltage [137].

One widely used instrumental setup for in-source CID is the combination of direct analysis in real time (DART) with high-resolution mass spectrometry (HRMS), such as time-of-flight (TOF) mass spectrometers $[138, \underline{139}]$. DART is a rapid, non-contact, ambient ionization technique that produces ions through gas-phase reactions of hot gas effluent from an atmospheric corona-to-glow discharge with reagent molecules and polar or nonpolar analytes $[\underline{140}, \underline{141}]$. The most common applications of DART-TOF with in-source CID are for the analysis of drugs, including synthetic cathinones [ $[\underline{32}, \underline{136}, \underline{142}, \underline{143}]$, opioids [144], cannabinoids [145], stimulants [146], and botanicals $[147, \underline{148]}$. DART with in-source CID and HRMS provides both molecular weight information 
from the pseudo-molecular ion and structural information from the in-source CID [149, 150]. Insource CID can also be employed on quadrupole time-of-flight (Q-TOF) instruments to enable product ion isolation and CID-TOF analysis to achieve pseudo-MS ${ }^{3}$ [37].

Whereas the use of in-source CID with HRMS has become widely accepted within the drug screening community, there is a void in the literature for the comparison between in-source CID and conventional beam-type CID. Power et al. examined the similarity between trapping-type CID spectra on an LTQ/Orbitrap instrument and in-source CID spectra collected with a single quadrupole [32]. However, their study isn't an apples-to-apples comparison because the mass analyzers were drastically different and might have different mass biases. For example, when comparing the spectra of in-source CID of five compounds on six different instruments, Bristow et al. noted that the geometry of the ionization source has noticeable effects on the degree of fragmentation and product ion distributions [151]. For the purpose of the present study, we kept the ESI source geometry and conditions constant and the TOF detection settings constant. The only variable was whether the ions were fragmented using in-source CID or beam-type CID. Although we have made some comparisons between in-source CID of ESI- and DART-generated precursor ions, those results are beyond the scope of the current work.

The goal of this study is to qualitatively analyze the product ion spectra generated with insource CID and beam-type CID for a series of fentanyl analogs and synthetic cathinones that have been previously well characterized $[\underline{106}, \underline{152}]$. The Q-TOF mass spectrometer allows simultaneous collection of full scan (containing in-source CID product ions) and beam-type CID product ion spectra, thereby allowing the comparison of mass spectra generated under conditions as similar as possible [138]. Understanding the relationship between these two commonly employed fragmentation techniques enables fundamental knowledge of fragmentation mechanisms from 
beam-type CID studies to be applied to in-source CID spectra. Finally, this study provides insight into the benefits and drawbacks of in-source CID relative to beam-type CID and the implications for forensic practitioners.

\subsection{Methods}

\subsubsection{Sample Preparation}

Four $N$-alkylated synthetic cathinones and two fentanyl analog standards were purchased through Cerilliant (Round Rock, TX, USA) including: methcathinone-d 3 ( $N$-alkyl deuterated), diethylpropion- $\mathrm{d}_{10}$ ( $N$-alkyl deuterated), pentylone- $\mathrm{d}_{3}$ ( $N$-alkyl deuterated), dibutylone- $\mathrm{d}_{3}$ (alkyl deuterated), alfentanil, and furanylfentanyl. Two additional fentanyl analogs were purchased through Cayman Chemical (Ann Arbor, MI, USA) including: ortho-methylfentanyl, and $\beta$ hydroxythiolfentanyl- $\mathrm{d}_{5}$ (perdeuterated on the amide). The synthetic cathinones were chosen based on the desire to have varying molecular weights and both $2^{\circ}$ and $3^{\circ}$ amines. The fentanyl analogs were chosen to represent analogs with substitutions on different positions of core fentanyl structure, consistent with our previous work on the influence of chemical modifications on the fragmentation behavior of fentanyl analogs [106]. All standards were prepared in a solution of 49\% HPLC grade methanol, $49 \%$ distilled water, and $2 \%$ acetic acid to a final concentration of approximately $100 \mathrm{ppm}$. The HPLC grade methanol was supplied by Fisher Scientific (Palo Alto, CA, USA) and the acetic acid was supplied by Acros Organics (Palo Alto, CA, USA).

\subsubsection{Instrumentation}

6.2.2.1 Agilent Technologies 6538 UHD Accurate-Mass Quadrupole Time-of-Flight (Q-TOF)

A dual ESI source was operated with a spray voltage of $3,500 \mathrm{~V}$ and a $300{ }^{\circ} \mathrm{C}$ nitrogen drying gas flow of $5 \mathrm{~L} / \mathrm{min}$ and a nebulizer flow of $30 \mathrm{psig}$. In-source CID spectra were collected using a 
skimmer setting of $65 \mathrm{~V}$ and fragmentor settings of varying from $95 \mathrm{~V}$ to $300 \mathrm{~V}$ to produce a range of in-source CID spectra. Likewise, the collision energy used for the beam-type CID portion of this study was varied from 15 to $35 \mathrm{eV}$ to providing a range of beam-type CID spectra. All beamtype CID spectra were collected with an isolation width of $1.3 \mathrm{Da}$ and the scan range was from $\mathrm{m} / \mathrm{z} 50$ to a value that exceeded the molecular mass by $\sim 50 \mathrm{Da}$. Ultra-pure nitrogen was used for the collision gas purchased through Matheson Tri-Gas (Fairmont, WV, USA).

\subsubsection{Thermo Scientific Velos Pro Linear Ion Trap (LIT)}

Supporting trapping-type CID experiments were also collected using a Thermo Scientific Velos Pro linear ion trap (LIT) mass spectrometer with a heated-electrospray ionization (HESI) source at $50{ }^{\circ} \mathrm{C}$. The spray voltage was $4,000 \mathrm{~V}$ with the nitrogen sheath gas flow set to 8 arbitrary units and the nitrogen auxiliary flow set to 5 arbitrary units. The mass spectrometer capillary temperature was set to $275^{\circ} \mathrm{C}$. The scan range and normalized collision energy (NCE) were specific for each compound and are labeled with each mass spectrum. The bath gas was ultra-pure helium from Matheson TRIGAS (Fairmont, WV, USA).

\subsubsection{Data Analysis}

MassHunter Qualitative Analysis B.05.00 was used for the Agilent Q-TOF data analysis. Microsoft Excel version 14 (Microsoft, Redmond, WA, USA) was used for the mass spectral plots and ChemDraw 16.0 (PerkinElmer, Waltham, MA, USA) was used to create the embedded structures. 


\subsection{Results and Discussiom}

\subsubsection{Fentanyl Analogs}

Figure 6.1 shows a comparison between beam-type CID and in-source CID spectra for orthomethylfentanyl. The beam-type CID spectrum (Figure 6.1a) was collected with a collision energy of $25 \mathrm{eV}$, a skimmer setting of $65 \mathrm{~V}$ and fragmentor setting of $250 \mathrm{~V}$. In contrast, the in-source CID spectra were collected with a collision energy of $0 \mathrm{eV}$, a skimmer setting of $65 \mathrm{~V}$ and fragmentor settings of $175 \mathrm{~V}$ (Figure 6.1b) and $300 \mathrm{~V}$ (Figure 6.1c). The $[\mathrm{M}+\mathrm{H}]^{+}$protonated precursor for ortho-methylfentanyl, $\mathrm{C}_{23} \mathrm{H}_{31} \mathrm{~N}_{2} \mathrm{O}^{+}$, is observed at $\mathrm{m} / z 351.2437$ (expected at $\mathrm{m} / z$ 351.2436; <1 ppm error) in Figure 6.1a. A sodiated adduct $\left([\mathrm{M}+\mathrm{Na}]^{+}, \mathrm{NaC}_{23} \mathrm{H}_{30} \mathrm{~N}_{2} \mathrm{O}^{+}\right)$is also evident at $m / z 373.2273$ (expected at $m / z$ 373.2255; 5 ppm error) in Figures 6.1b and 6.1c. Figure 6.1a shows the beam-type CID spectrum with product ions at $m / z, 230.1590\left(\mathrm{C}_{15} \mathrm{H}_{20} \mathrm{NO}^{+}\right.$expected at $m / z$ 230.1544; 20 ppm error), $m / z$ 188.1483 $\left(\mathrm{C}_{13} \mathrm{H}_{18} \mathrm{~N}^{+}\right.$expected at $m / z$ 188.1439; 23 ppm error), $m / z \quad 146.0990\left(\mathrm{C}_{10} \mathrm{H}_{12} \mathrm{~N}^{+}\right.$expected at $m / z, 146.0969 ; 14 \mathrm{ppm}$ error $), m / z, 134.0983\left(\mathrm{C}_{9} \mathrm{H}_{12} \mathrm{~N}^{+}\right.$ expected at $m / z$ 134.0969; 10 ppm error $)$ and $m / z 105.0718\left(\mathrm{C}_{8} \mathrm{H}_{9}{ }^{+}\right.$expected at $\mathrm{m} / \mathrm{z} 105.0704 ; 13$ ppm error), which is consistent with our previous work [106]. For ortho-methylfentanyl, all of the product ions formed in beam-type CID are found in the in-source CID spectrum, but the reverse is not true. The in-source CID spectrum shows a phenylium peak at $m / z 77.0417$ that is negligible in the beam-type CID spectrum. The phenylium ion at $\mathrm{m} / \mathrm{z} 77.0417$ either derives from a higher energy direct cleavage of the precursor or from a simple neutral loss of $\mathrm{C}_{2} \mathrm{H}_{4}$ from the phenylethyl ion at $m / z$ 105.0718.

The fragmentor setting of $175 \mathrm{~V}$ in Figure 6.1b provided inefficient fragmentation and weak product ion signals. Low-abundance in-source CID product ions are observed at $\mathrm{m} / \mathrm{z} 188.1483$ for $\mathrm{C}_{13} \mathrm{H}_{18} \mathrm{~N}^{+}$(expected at $\mathrm{m} / \mathrm{z}$ 188.1439; 23 ppm error) and $\mathrm{m} / \mathrm{z} 105.0718$ for $\mathrm{C}_{8} \mathrm{H}_{9}{ }^{+}$(expected at $\mathrm{m} / z$ 
105.0704; 13 ppm error). The phenethylpiperidine ion expected at $m / z \quad 188.1439$ has been described extensively for fentanyl, including the presence of isobaric species formed through the loss of the $N$-phenylpropanamide moiety directly or through the loss of methylketene followed by the loss of aniline [97, $\underline{121]}$.

The presence of the phenethylpiperidine ion at $\mathrm{m} / \mathrm{z} 188.1483$ and the phenylethyl ion at $\mathrm{m} / \mathrm{z}$ 105.0718 are consistent with previous literature on in-source CID of fentanyl and fentanyl analogs [119]. The actual structure of the fragment at $\mathrm{m} / \mathrm{z} 105.0718$ could also be a methyl-tropylium or phenonium ion [153]. The ion at $m / z, 98.9782$ in Figure 6.1b has a negative mass defect that is not consistent with any viable fragment of ortho-methylfentanyl and must therefore derive from an impurity or background contamination. The same ion was observed in other full-scan and low energy in-source CID spectra, and the most reasonable identity-based on the likelihood of occurrence and accurate mass - is $\mathrm{H}_{3} \mathrm{SO}_{4}{ }^{+}$(expected at $\mathrm{m} / \mathrm{z}$ 98.9747; 35 ppm error). However, the chemical identity of the ion at $m / z 98.9782$ has not been confirmed.

In contrast to the low energy in-source CID spectrum in Figure 6.1b, the higher energy insource CID spectrum in Figure 6.1c provides more abundant low mass ions, including the readily observed phenylium product ion, $\mathrm{C}_{6} \mathrm{H}_{5}{ }^{+}$, at $m / z 77.0417$ (expected at $\mathrm{m} / \mathrm{z} 77.0391 ; 34$ ppm error). As expected, larger fragmentor voltages enhanced the fragmentation efficiencies and decreased the precursor ion abundance. 

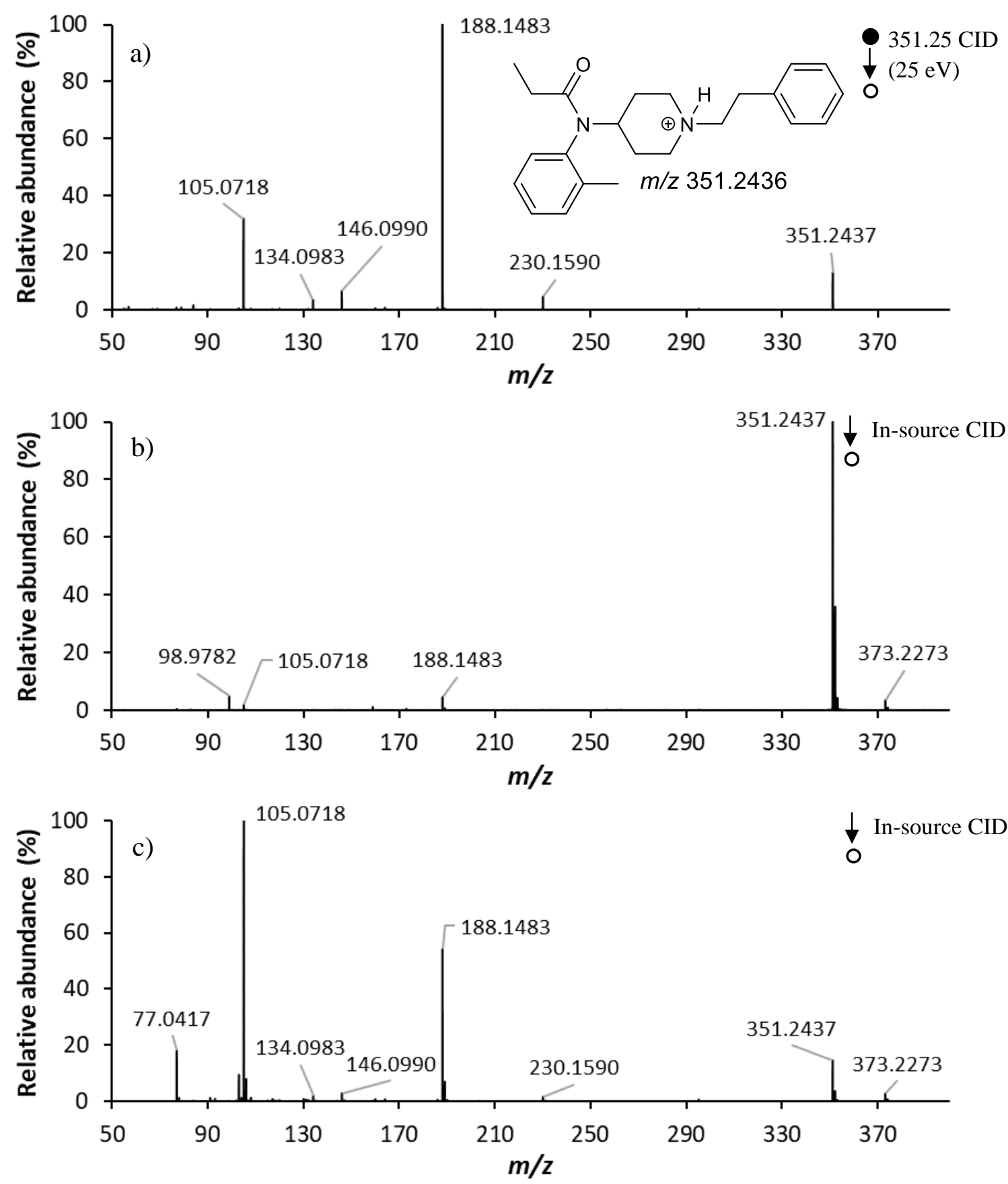

Figure 6.1. Comparison of beam-type CID (top) and in-source CID (middle and bottom) for protonated ortho-methylfentanyl. For all spectra, the skimmer setting was held at $65 \mathrm{~V}$. For beam-type CID, the fragmentor setting was $250 \mathrm{~V}$ with a collision energy of $25 \mathrm{eV}$. For insource CID, the fragmentor settings were $175 \mathrm{~V}$ and $300 \mathrm{~V}$, for b) and c), respectively with the collision energy set to $0 \mathrm{eV}$. 
The $[\mathrm{M}+\mathrm{H}]^{+}$protonated precursor of furanylfentanyl, $\mathrm{C}_{24} \mathrm{H}_{27} \mathrm{~N}_{2} \mathrm{O}_{2}{ }^{+}$, is observed at $\mathrm{m} / \mathrm{z}$ 375.2134, (expected at $m / z, 375.2072 ; 16$ ppm error) in Figure 6.2. A sodiated adduct ([M+Na $]^{+}$, $\mathrm{NaC}_{24} \mathrm{H}_{26} \mathrm{~N}_{2} \mathrm{O}_{2}{ }^{+}$) is also evident at $\mathrm{m} / z 397.1914$ (expected at $\mathrm{m} / \mathrm{z} 397.1891 ; 6 \mathrm{ppm}$ error) in Figures 6.2b and 6.2c. Figure 6.2a is the beam-type CID spectrum collected with a collision energy of $25 \mathrm{eV}$, fragmentor setting of $250 \mathrm{~V}$ and skimmer setting of $65 \mathrm{~V}$. In contrast to Figure 6.1, the observed product ion distribution is noticeably absent of any intermediate of significant abundance between the precursor ion and the phenethylpiperidine ion at $m / z$ 188.1471. At higher energies, the electron-withdrawing characteristics of the furyl group for furanylfentanyl tend to favor the direct loss of the $\mathrm{N}$-phenylalkylamide moiety and drive facile secondary fragmentation [106]. The product ions at $m / z 188.1471\left(\mathrm{C}_{13} \mathrm{H}_{18} \mathrm{~N}^{+}\right.$, expected at $\mathrm{m} / \mathrm{z} 188.1439 ; 17 \mathrm{ppm}$ error $), \mathrm{m} / \mathrm{z}$ $146.1001\left(\mathrm{C}_{10} \mathrm{H}_{12} \mathrm{~N}^{+}\right.$expected at $\mathrm{m} / z$ 146.0969; $22 \mathrm{ppm}$ error $), \mathrm{m} / z .134 .1000\left(\mathrm{C}_{9} \mathrm{H}_{12} \mathrm{~N}^{+}\right.$expected at $\mathrm{m} / \mathrm{z} 134.0969 ; 24 \mathrm{ppm}$ error) and $\mathrm{m} / \mathrm{z} 105.0713\left(\mathrm{C}_{8} \mathrm{H}_{9}{ }^{+}\right.$expected at $\mathrm{m} / \mathrm{z} 105.0704 ; 9 \mathrm{ppm}$ error $)$ are consistent with the previous literature [94, 97, 105].

In contrast, Figures 6.2b and 6.2c show in-source CID generated mass spectra collected with a collision energy of $0 \mathrm{eV}$, a skimmer setting of $65 \mathrm{~V}$ and fragmentor setting of $165 \mathrm{~V}$ and $285 \mathrm{~V}$, respectively. Figure 6.2b provides a low abundance of product ions because the fragmentor offset is too low to achieve efficient CID. The poor spectrum shows that it is difficult or impossible to obtain low-energy in-source CID spectra with acceptable CID efficiencies. The presence of the phenethylpiperidine ion, $\mathrm{C}_{13} \mathrm{H}_{18} \mathrm{~N}^{+}$, at $\mathrm{m} / z 188.1471$ (expected at $\mathrm{m} / z \mathbf{z} 188.1439 ; 17 \mathrm{ppm}$ error) and phenylethyl (or phenonium or methyl-tropylium ion [153]), $\mathrm{C}_{8} \mathrm{H}_{9}{ }^{+}$, at $\mathrm{m} / \mathrm{z} 105.0713$ (expected at $\mathrm{m} / \mathrm{z}$ 105.0704; $9 \mathrm{ppm}$ error) has been reported previously in literature for furanylfentanyl [154]. As the fragmentor voltage is increased, the low-mass product ions increase in abundance relative to the precursor ion signal (Figure 6.2c). 
One notable difference between the in-source CID spectrum and beam-type CID spectrum of furanylfentanyl is the abundance of the phenylium ion at $\mathrm{m} / \mathrm{z} 77.0391$ for beam-type CID, which, as discussed above, could be a higher energy direct cleavage product or a consecutive fragment. The accurate mass measurements indicate that the elemental composition at $m / z 105.0713$ is $\mathrm{C}_{8} \mathrm{H}_{9}{ }^{+}$, which is consistent with a phenylethyl (or methyl-tropylium or phenonium ion [153]) structure from the $N$-phenylethyl group on the piperidine nitrogen. The expected pathway to the phenylethyl cation is through the charge-directed $\alpha$-cleavage of the phenylethyl group from the 1-(2phenylethyl)-2,3,4,5-tetrahydropyridium ion at $m / z 188.1471$ [97, 121]. 

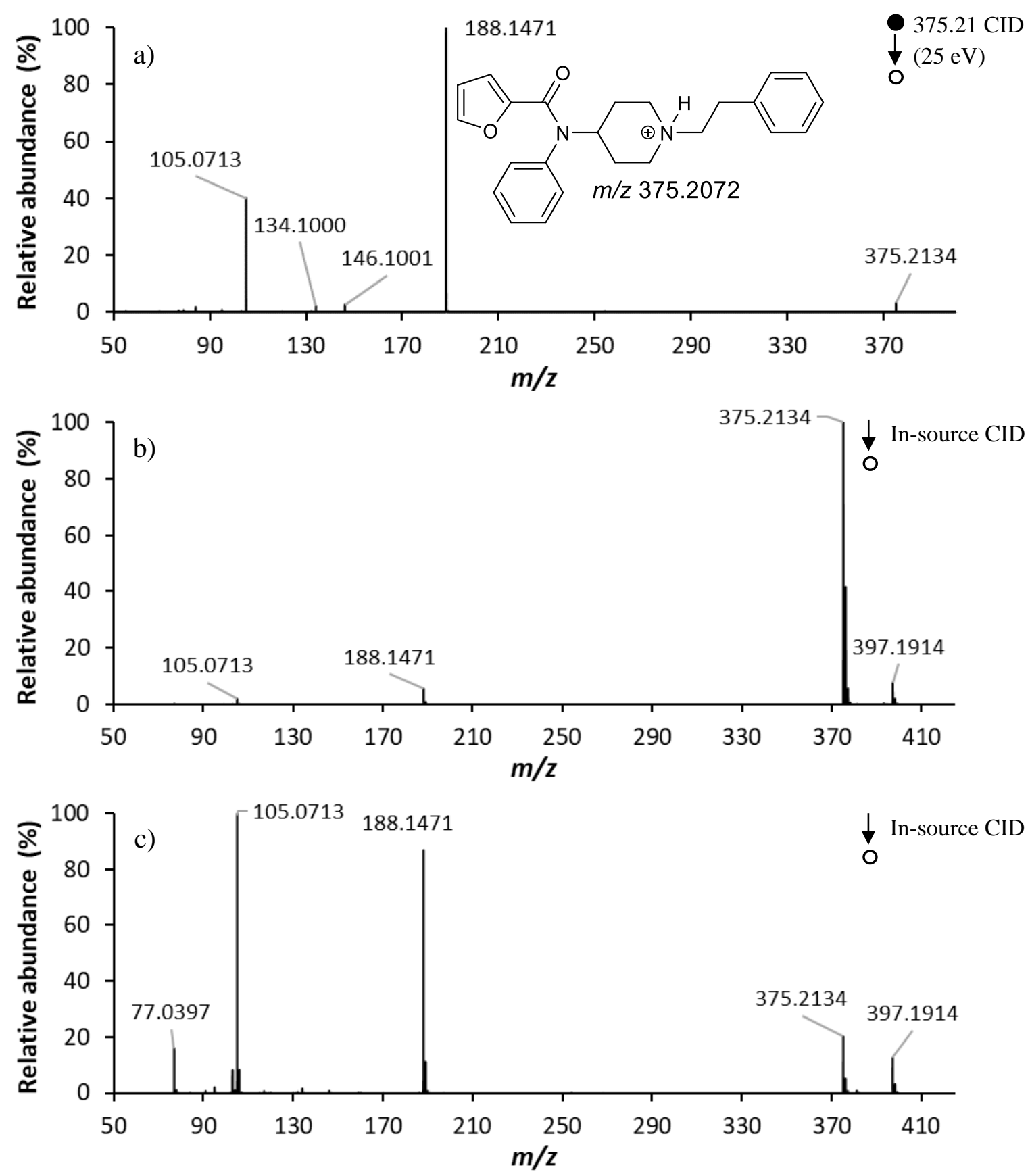

Figure 6.2. Comparison of beam-type CID (top) and in-source CID (middle and bottom) for protonated furanylfentanyl. For all spectra, the skimmer setting was held at $65 \mathrm{~V}$. For beam-type $\mathrm{CID}$, the fragmentor setting was $225 \mathrm{~V}$ with a collision energy of $25 \mathrm{eV}$. For in-source CID, the fragmentor settings were $165 \mathrm{~V}$ and $285 \mathrm{~V}$, for b) and c), respectively with the collision energy set to $0 \mathrm{eV}$. 
Figure 6.3 shows a comparison between the beam-type CID spectra and in-source CID spectrum for alfentanil collected at different collision energies. Figures $\mathbf{6 . 3 a}$ and $\mathbf{6 . 3 b}$ were collected with collision energies of $15 \mathrm{eV}$ and $25 \mathrm{eV}$, respectively. Given the product ion distribution and the pattern and extent of in-source CID fragmentation in Figure 6.3c, in-source CID collected with a collision energy of $0 \mathrm{eV}$, a skimmer setting of $65 \mathrm{~V}$ and a fragmentor setting of $285 \mathrm{~V}$ appears to produce similar product ion spectra to beam-type CID with collision energies between 15-25 eV (Figure 6.3a \& 6.3b). Under the in-source CID conditions there is a wealth of product ion information in addition to the presence of the $[\mathrm{M}+\mathrm{H}]^{+}$protonated precursor, $\mathrm{C}_{21} \mathrm{H}_{33} \mathrm{~N}_{6} \mathrm{O}_{3}{ }^{+}$, at $m / z 417.2628$ (expected at $m / z 417.2614 ; 3$ ppm error) and the $[\mathrm{M}+\mathrm{Na}]^{+}$precursor, $\mathrm{NaC}_{21} \mathrm{H}_{32} \mathrm{~N}_{6} \mathrm{O}_{3}{ }^{+}$, at $m / z 439.2480$ (expected at $m / z$ 439.2433; 11 ppm error). The major in-source CID generated product ions at $m / z 385.2381\left(\mathrm{C}_{20} \mathrm{H}_{29} \mathrm{~N}_{6} \mathrm{O}_{2}{ }^{+}\right.$expected at $m / z 385.2351 ; 8$ ppm error), $m / z 314.1892\left(\mathrm{C}_{18} \mathrm{H}_{24} \mathrm{~N}_{3} \mathrm{O}_{2}{ }^{+}\right.$expected at $m / z$ 314.1868; 7 ppm error $), m / z 268.1831\left(\mathrm{C}_{12} \mathrm{H}_{22} \mathrm{~N}_{5} \mathrm{O}_{2}{ }^{+}\right.$ expected at $m / z, 268.1773 ; 22$ ppm error $), m / z$ 197.1296 $\left(\mathrm{C}_{10} \mathrm{H}_{17} \mathrm{~N}_{2} \mathrm{O}_{2}{ }^{+}\right.$expected at $m / z$ 197.1290; 3 ppm error) and $m / z$ 165.1064 $\left(\mathrm{C}_{9} \mathrm{H}_{13} \mathrm{~N}_{2} \mathrm{O}^{+}\right.$expected at $\mathrm{m} / \mathrm{z} 165.1027 ; 23$ ppm error $)$ are all in agreement with the beam-type CID spectra. The observed product ions are consistent with previous literature for substitution at the 4-position of the pipiderine ring, as seen by the neutral losses of methanol (i.e. at $m / z, 385.2381$ ) and $N$-phenylpropanamide (i.e. at $m / z$ 268.1831) [106, 122$]$. 

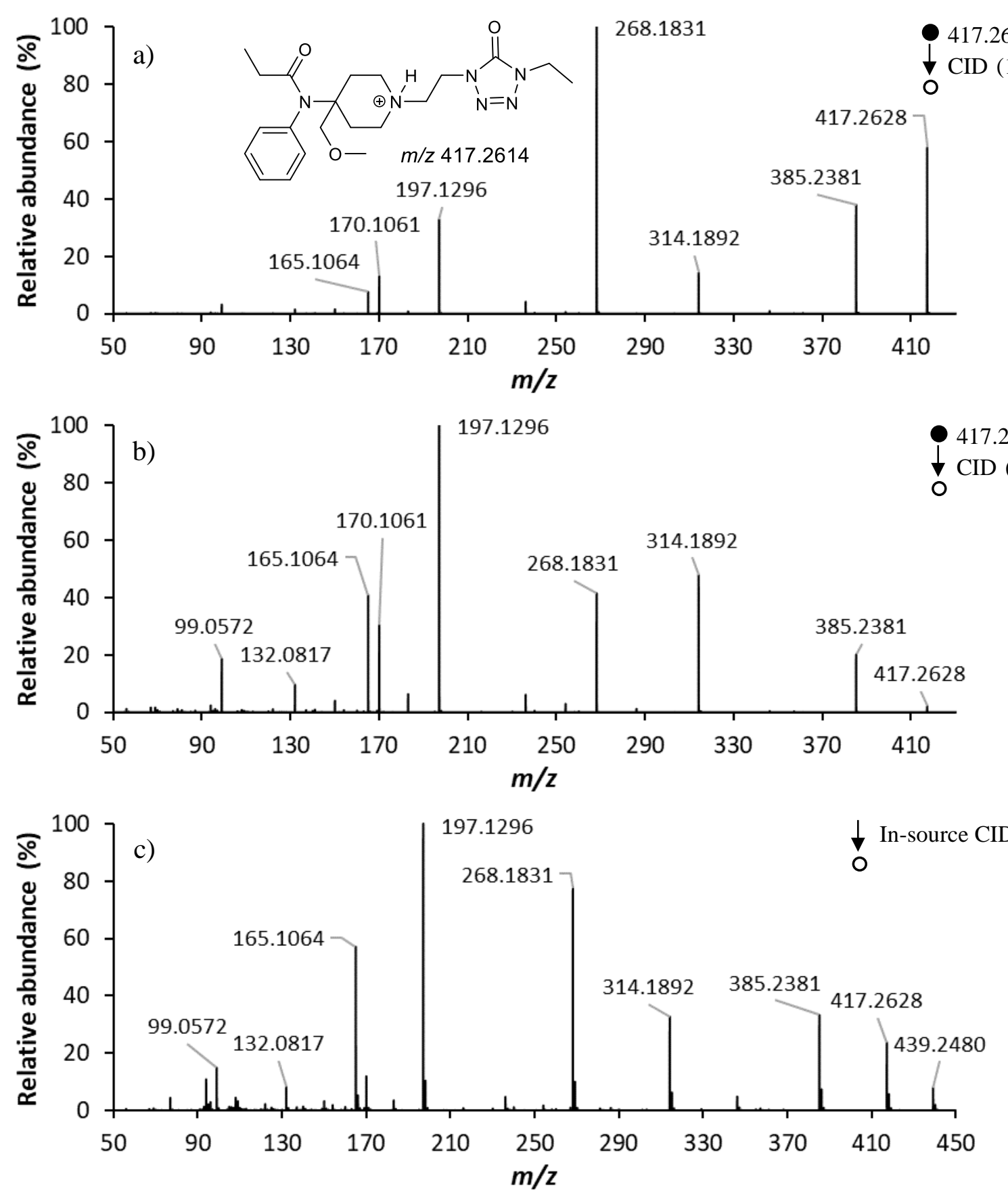

Figure 6.3. Comparison of beam-type CID (top and middle) and in-source CID (bottom) for protonated alfentanil. For all spectra, the skimmer setting was held at $65 \mathrm{~V}$. For beam-type CID, the fragmentor setting was $225 \mathrm{~V}$ with collision energies of $15 \mathrm{eV}$ and $25 \mathrm{eV}$ for panels a) and b), respectively. For in-source CID, the fragmentor setting was $285 \mathrm{~V}$ with a collision energy of $0 \mathrm{eV}$. 
Figure 6.4 compares spectra from beam-type CID and in-source CID of $\beta$ hydroxythiolfentanyl-d5. The beam-type CID spectra in Figures 6.4a and 6.4b were collected with a skimmer setting of $65 \mathrm{~V}$, a fragmentor setting of $225 \mathrm{~V}$ and collision energies of $15 \mathrm{eV}$ and 25 $\mathrm{eV}$, respectively. As is typical for both beam-type and in-source CID, the product ion distribution shifts towards lower masses at higher collision energies (Figure 6.4b) relative to lower collision energies (Figure 6.4a) because of sequential neutral losses and access to pathways with higher dissociation energies. The base peak of Figure 6.4a is $m / z$ 346.2021, which is consistent with the neutral loss of $\mathrm{H}_{2} \mathrm{O}$ from the $[\mathrm{M}+\mathrm{H}]^{+}$protonated precursor. This intermediate ion at $\mathrm{m} / \mathrm{z} 346.2021$ fragments into the products at $\mathrm{m} / \mathrm{z} 286.1511$ and $\mathrm{m} / \mathrm{z}$ 192.0895, which have been described previously [106].

The in-source CID spectrum (Figure 6.4c) was collected with a collision energy of $0 \mathrm{eV}$, a skimmer setting of $65 \mathrm{~V}$ and a fragmentor setting of $285 \mathrm{~V}$. Figure 6.4c contains both the $[\mathrm{M}+\mathrm{H}]^{+}$ protonated precursor, $\mathrm{C}_{20} \mathrm{H}_{22} \mathrm{D}_{5} \mathrm{~N}_{2} \mathrm{O}_{2} \mathrm{~S}^{+}$, at $\mathrm{m} / z 364.2114$ (expected at $\mathrm{m} / z$ 364.2102; 3 ppm error) and the $[\mathrm{M}+\mathrm{Na}]^{+}$precursor, $\mathrm{NaC}_{20} \mathrm{H}_{21} \mathrm{D}_{5} \mathrm{~N}_{2} \mathrm{O}_{2} \mathrm{~S}^{+}$, at $\mathrm{m} / z .386 .1955$ (expected at $\mathrm{m} / \mathrm{z}$ 386.1921; 9 ppm error). The fragmentor setting of $285 \mathrm{~V}$ provides sufficient collisional activation to generate multiple diagnostic product ions, including $m / z 346.2021$ for $\mathrm{C}_{20} \mathrm{H}_{20} \mathrm{D}_{5} \mathrm{~N}_{2} \mathrm{OS}^{+}$(expected at $\mathrm{m} / z$ 346.1996; 7 ppm error), $m / z 286.1511$ for $\mathrm{C}_{17} \mathrm{H}_{20} \mathrm{DN}_{2} \mathrm{~S}^{+}$(expected at $m / z$ 286.1487; 8 ppm error), $m / z 210.0982$ for $\mathrm{C}_{11} \mathrm{H}_{16} \mathrm{NOS}^{+}$(expected at $\mathrm{m} / \mathrm{z}$ 210.0952; $14 \mathrm{ppm}$ error), $\mathrm{m} / \mathrm{z} 192.0895$ for $\mathrm{C}_{11} \mathrm{H}_{14} \mathrm{D}_{5} \mathrm{NS}^{+}$(expected at $m / z$ 192.0846; 26 ppm error), $\mathrm{m} / z 111.0295$ for $\mathrm{C}_{6} \mathrm{H}_{7} \mathrm{~S}^{+}$(expected at $\mathrm{m} / z$ 111.0268; 23 ppm error) and $m / z$ 97.0135 for $\mathrm{C}_{5} \mathrm{H}_{5} \mathrm{~S}^{+}$(expected at $\mathrm{m} / z$ 97.0111; 25 ppm error). 

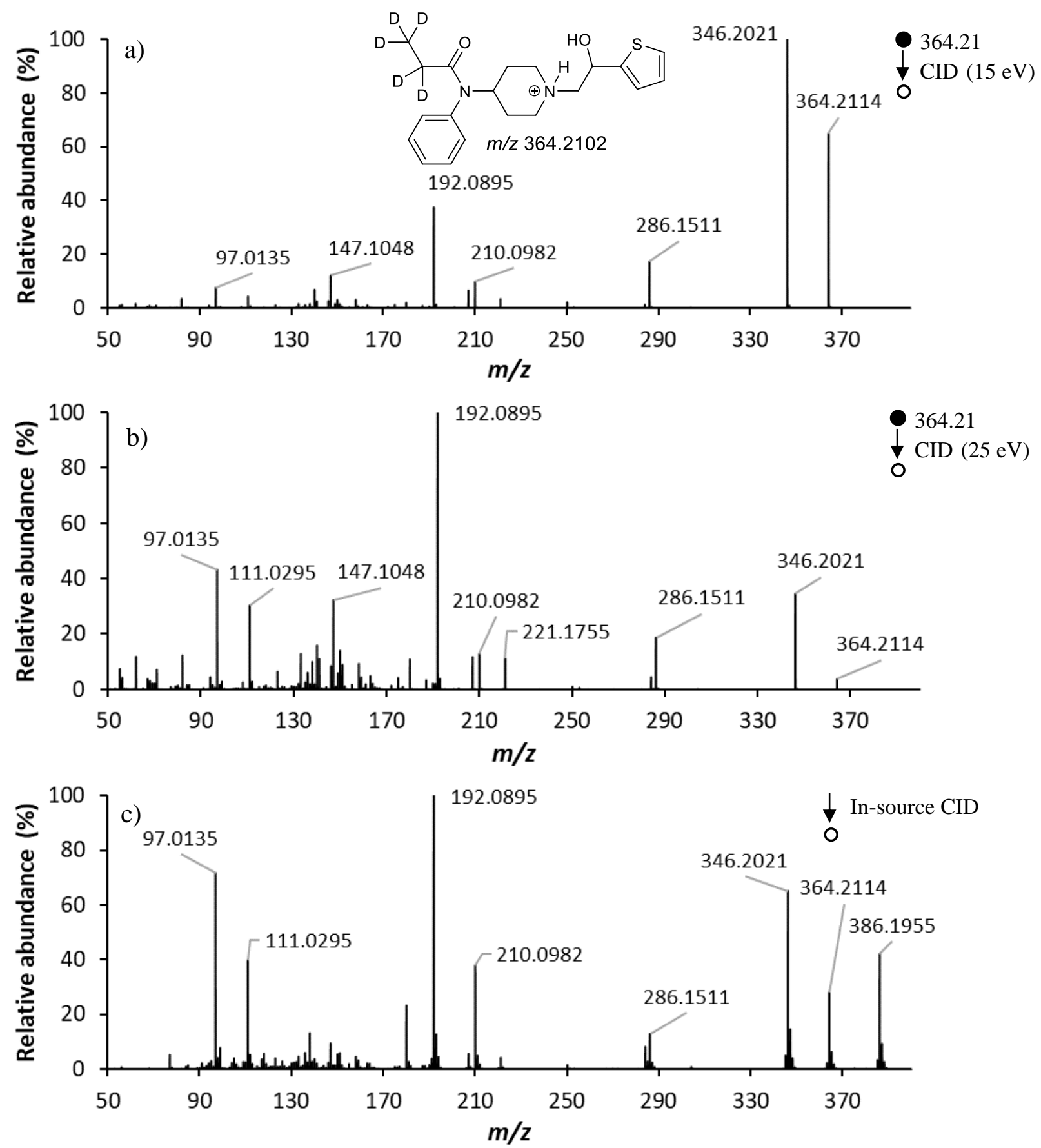

Figure 6.4. Comparison of beam-type CID (top and middle) and in-source CID (bottom)for protonated $\beta$-hydroxythiolfentanyl- $\mathrm{d}_{5}$. For all spectra, the skimmer setting was held at $65 \mathrm{~V}$. For beam-type CID, the fragmentor setting was $225 \mathrm{~V}$ with collision energies of $15 \mathrm{eV}$ and $25 \mathrm{eV}$, for a) and b), respectively. For in-source CID, the fragmentor settings was $285 \mathrm{~V}$ with a collision energy of $0 \mathrm{eV}$. 


\subsubsection{Discussion of Fentanyl Analogs}

The comparison between in-source CID and beam-type CID for fentanyl analogs reveals a great deal of similarity between the observed product ion spectra. In general, the abundant product ions are conserved between the two activation techniques, and there is general agreement between the spectra. A careful assessment also reveals that the in-source CID spectra tend to be noisier relative to the beam-type CID spectra, and that there are usually distinguishable differences in the relative abundance of selected product ions, which is observed as both ion drop-in and ion dropout. For example, the in-source CID spectrum of ortho-methylfentanyl in Figure 6.1b shows the presence of a product ion at $\mathrm{m} / \mathrm{z} 98.9782$ that is not present in the beam-type CID spectrum in Figure 6.1a. We interpret this observation to imply that $\mathrm{m} / \mathrm{z} 98.9782$ must arise from an alternative precursor than ortho-methylfentanyl because there are no reported pathways to this fragment mass in the literature. Furthermore, the product ion at $\mathrm{m} / z 98.9782$ is not present in the higher energy in-source CID spectrum (Figure 6.1c), which indicates that the ion has probably undergone collisional activation in Figure 6.1c. Such observations are significant because the fentanyl analogs in this study were standards and thus have minimal background interferences, unlike casework samples that would contain mixtures of drugs, cutting agents, adulterants or complex biological matrices.

When comparing in-source CID and beam-type CID in Figures 6.1-6.3, subtle differences in the relative abundance of peaks with comparable $\mathrm{m} / \mathrm{z}$ values indicate fundamental differences in internal energy deposition rates rather than mass bias or other effects if the peaks were spaced further apart. For example, the in-source CID spectrum of alfentanil (Figure 6.3c) shows a peak at $m / z, 170.1061$ (expected at $m / z, 170.1042$ for $\mathrm{C}_{6} \mathrm{H}_{12} \mathrm{~N}_{5} \mathrm{O}^{+} ; 12$ ppm error) that is only $\sim 20 \%$ the abundance of the fragment at $\mathrm{m} / z 165.1064$ (expected at $\mathrm{m} / z .165 .1027$ for $\mathrm{C}_{9} \mathrm{H}_{13} \mathrm{~N}_{2} \mathrm{O}^{+} ; 22 \mathrm{ppm}$ error). At the higher beam-type CID amplitude of $25 \mathrm{eV}$ in Figure 6.3b, the peak at $\mathrm{m} / \mathrm{z} 170.1061$ 
is $\sim 80 \%$ the abundance of the peak at $\mathrm{m} / z$ 165.1064. In contrast, the lower energy spectrum in Figure 6.3a — with a collision energy of $15 \mathrm{eV}$ - provides a peak at $\mathrm{m} / \mathrm{z} 170.1061$ that is $130 \%$ the abundance than the peak at $m / z$ 165.1064. In the slow heating conditions of trapping-type CID, the same peak at $\mathrm{m} / z 170$ was $\sim 140 \%$ the abundance of the peak at $\mathrm{m} / \mathrm{z} 165$ [106]. The ratio of abundances at $\mathrm{m} / \mathrm{z}$ 165:170 therefore varies from $\sim 0.75: 1$ for in trapping-based CID and lowenergy beam-type CID to $1.25: 1$ at higher energy beam-type CID and $\sim 5: 1$ for in-source CID. Based on $\mathrm{MS}^{\mathrm{n}}$ data collected on the LIT, the product ions at $\mathrm{m} / \mathrm{z} 170$ and $\mathrm{m} / \mathrm{z} 165$ form via consecutive neutral losses through several different fragmentation pathways, including the abundant intermediate ion at $\mathrm{m} / \mathrm{z} 268$, which is formed through the loss of neutral $\mathrm{N}$ phenylpropanamide from the protonated precursor. From the intermediate ion at $\mathrm{m} / \mathrm{z} 268$, the product ion at $\mathrm{m} / \mathrm{z} 165$ forms through the loss of $\mathrm{C}_{2} \mathrm{H}_{5} \mathrm{~N}_{3}$ (i.e. $\mathrm{m} / \mathrm{z}$ 197) followed by the loss of methanol, whereas the product ion at $m / z, 170$ forms through the loss of $\mathrm{C}_{6} \mathrm{H}_{10} \mathrm{O}$ from a piperidine ring cleavage. The abundance of $m / z 165$ correlates with the abundance of its precursor at $m / z$ 197, with both product ions $(\mathrm{m} / z 165$ and $\mathrm{m} / \mathrm{z}$ 197) gaining prominence at elevated CID energies in beam-type CID (Figure 6.3b) relative to trapping-type CID [106]. Based on the comparison between in-source CID (Figure 6.3c), beam-type CID (Figures 6.3a and 6.3b) and trapping-type CID [106], in-source CID provides the greatest relative abundance of peaks at $m / z 165$ and 197. Because the relative energies and activation barriers for these pathways are not known, we can only speculate that the product ion at $m / z 170$ has a lower activation barrier than $m / z 165$.

In the slow heating conditions of trapping-type CID of $\beta$-hydroxythiolfentanyl- $\mathrm{d}_{5}$, the peak at $m / z 207$ was slightly more abundant than the peak at $m / z 210$ [106]. The beam-type CID spectra in Figures 6.4a and 6.4b provide an accurate mass of $m / \mathrm{z} 207.1540$ (expected at $\mathrm{m} / \mathrm{z} 207.1540$ for $\mathrm{C}_{13} \mathrm{H}_{11} \mathrm{D}_{5} \mathrm{NO}^{+} ;<1 \mathrm{ppm}$ error), which forms through competing pathways through either the 
loss of the hydroxymethylthiol to form the intermediate at $\mathrm{m} / \mathrm{z} 250$ followed by the loss of a $\mathrm{C}_{2} \mathrm{NH}_{5}$ neutral from the piperidine ring or through the loss of $\mathrm{H}_{2} \mathrm{O}$, followed by the loss of the deuterated $N$-phenylpropanamide moiety. These pathways were confirmed in $\mathrm{MS}^{3}$ experiments with the ion trap [106]. In contrast to consecutive low-energy rearrangements leading to $\mathrm{m} / \mathrm{z}$ 207.1540, the product ion at $\mathrm{m} / \mathrm{z} 210.0982$ has an elemental composition $\mathrm{C}_{11} \mathrm{H}_{16} \mathrm{NOS}^{+}$(expected at $m / 2$ 210.0952; $14 \mathrm{ppm}$ error), and forms via cleavage between the aniline nitrogen and the piperidine ring in a single step, so is probably kinetically favored. The beam-type CID spectra show similar abundances for the two product ions at $\mathrm{m} / \mathrm{z} 207.1540$ and $\mathrm{m} / \mathrm{z} 210.0982$, whereas the in-source CID spectrum in Figure 6.4a shows that the peak at $\mathrm{m} / z 210.0982$ is considerably more abundant (by a factor of $\sim 7 \mathrm{x}$ ). Again, these findings show that the in-source CID spectrum in Figure 4a provides more rapid heating and kinetically-favored single-cleavage product ions relative to beam-type CID experiments or the trapping-type CID experiments of our previous work [33].

\subsubsection{Synthetic Cathinones}

Figure 6.5 shows the product ion spectra for methcathinone-d 3 collected with beam-type CID and in-source CID. The perdeuterated methyl group is in the $N$-alkyl position. Methcathionone- $\mathrm{d}_{3}$ is a $2^{\circ}$ amine, $N$-alkylated, synthetic cathinone and has a relatively small molecular weight compared to other cathinones and fentanyl analogs. The beam-type CID in Figure 6.5a was collected with a collision energy of $25 \mathrm{eV}$, a skimmer setting of $65 \mathrm{~V}$ and a fragmentor setting of $175 \mathrm{~V}$. The in-source CID spectra were collected with a collision energy of $0 \mathrm{eV}$, a skimmer setting of $65 \mathrm{~V}$ and fragmentor settings of $175 \mathrm{~V}$ (Figure 6.5b) and $255 \mathrm{~V}$ (Figure 6.5c), respectively. Figure 6.5b shows the dominant loss of $\mathrm{H}_{2} \mathrm{O}$ observed at $\mathrm{m} / z 149.1160\left(\mathrm{C}_{10} \mathrm{H}_{9} \mathrm{D}_{3} \mathrm{~N}^{+}\right.$expected at $m / z 149.1154 ; 4 \mathrm{ppm}$ error) from the $[\mathrm{M}+\mathrm{H}]^{+}$protonated precursor at $m / z .167 .1295\left(\mathrm{C}_{10} \mathrm{H}_{11} \mathrm{D}_{3} \mathrm{NO}^{+}\right.$ 
expected at $m / z$ 167.1260; 21 ppm error). This fragmentation behavior is consistent with previous literature for $N$-alkylated synthetic cathinones $[\underline{15}, \underline{17}, \underline{28}, \underline{36}, \underline{77]}$. Note that, unlike the fentanyl analogs, neither methcathinone- $\mathrm{d}_{3}$, nor any of the other synthetic cathinones show sodiated adducts $\left([\mathrm{M}+\mathrm{Na}]^{+}\right)$in the full scan mass spectra. The lack of sodiation likely is related to the relative sodium affinity of synthetic cathinones relative to fentanyl analogs, but could also be related to differences in the sodium impurities of the purchased standards.

Figure 6.5c shows that, at higher acceleration potentials of in-source CID, the product ion distribution shifts towards lower masses, likely because the smaller ions are thermodynamically less stable than large ions and have higher dissociation thresholds. The ions at smaller $\mathrm{m} / \mathrm{z}$ values could also derive from multiple neutral losses. The major product ions observed from beam-type CID are consistent with the major product ions observed for in-source CID, including product ions at $m / z 149.1160$ for $\mathrm{C}_{10} \mathrm{H}_{9} \mathrm{D}_{3} \mathrm{~N}^{+}$(expected at $\mathrm{m} / z$ 149.1154; 4 ppm error), $\mathrm{m} / z 134.0960$ for $\mathrm{C}_{9} \mathrm{H}_{6} \mathrm{D}_{3} \mathrm{~N}^{+}$(expected at $m / z$ 134.0920; 30 ppm error), $m / z, 131.0744$ for $\mathrm{C}_{9} \mathrm{H}_{9} \mathrm{~N}^{+}$(expected at $m / z$ 131.0734; 8 ppm error) and $m / z 105.0713$ for $\mathrm{C}_{8} \mathrm{H}_{9}{ }^{+}$(expected at $\mathrm{m} / z$ 105.0704; 9 ppm error). Two product ions of note are $m / z 134.0920$ and $m / z$ 131.0744, which are formed through the loss of a radical methyl group $\left({ }^{\circ} \mathrm{CH}_{3}\right)$ from the aliphatic chain and a radical deuterated methyl group $\left({ }^{\circ} \mathrm{CD}_{3}\right)$ from the $N$-alkyl chain, respectively. The presence of even-electron intermediates fragmenting into odd-electron product ions has been reported before for $N$-alkylated synthetic cathinones [주, $\underline{37}$, 152].

Similar to the fentanyl analogs, the in-source CID spectra and beam-type CID spectra occasionally show significant differences in relative ion abundances for peaks that are close together and therefore these differences in abundance are not caused by mass bias. For example, in the beam-type CID spectrum of methcathinone- $d_{3}$ in Figure 6.5a, the peak at $m / z, 131.0744$ is 
$\sim 39 \%$ more abundant than the peak at $\mathrm{m} / \mathrm{z}$ 130.0670. However, although the higher energy insource CID spectrum in Figure 6.5c shares overall spectral similarity with the beam-type CID spectrum, the relative abundance of the peak at $\mathrm{m} / z 131.0744$ is only $~ 50 \%$ the abundance of the peak at $\mathrm{m} / \mathrm{z}$ 130.0670. Previous work indicates that the product ion at $\mathrm{m} / \mathrm{z} 131.0744$ is a distonic radical cation formed via the loss of the methyl group from the $N$-alkyl position $[\underline{31}, \underline{34}, \underline{41]}$. Without the benefit of isotope labeling_Bijlsma et al. assumed that the methyl radical was lost from the aliphatic chain [31]. However, per-deuteration on the $N$-methyl group shows that the intermediate at $m / z, 149.1160$ can evidently lose either ${ }^{\circ} \mathrm{CH}_{3}(15 \mathrm{Da})$ from the aliphatic chain to form the product at $\mathrm{m} / z 134.0960$ or ${ }^{\circ} \mathrm{CD}_{3}(18 \mathrm{Da})$ from the $N$-methyl position to form the product at $\mathrm{m} / \mathrm{z} 131.0744$ with approximately equal preference [34]. The formation of an alkylphenone at $\mathrm{m} / z 133.0960$ through the loss of the $N$-methyl moiety is generally unfavorable in all the spectra, whereas the additional radical loss of ${ }^{\circ} \mathrm{H}(1 \mathrm{Da})$ from the distonic radical intermediate at $\mathrm{m} / \mathrm{z}$ 131.0744 to give the even-electron product ion at $\mathrm{m} / \mathrm{z}, 130.0670$ is kinetically favored at higher internal energies, as demonstrated by its greater abundance in the highest energy in-source CID conditions. 

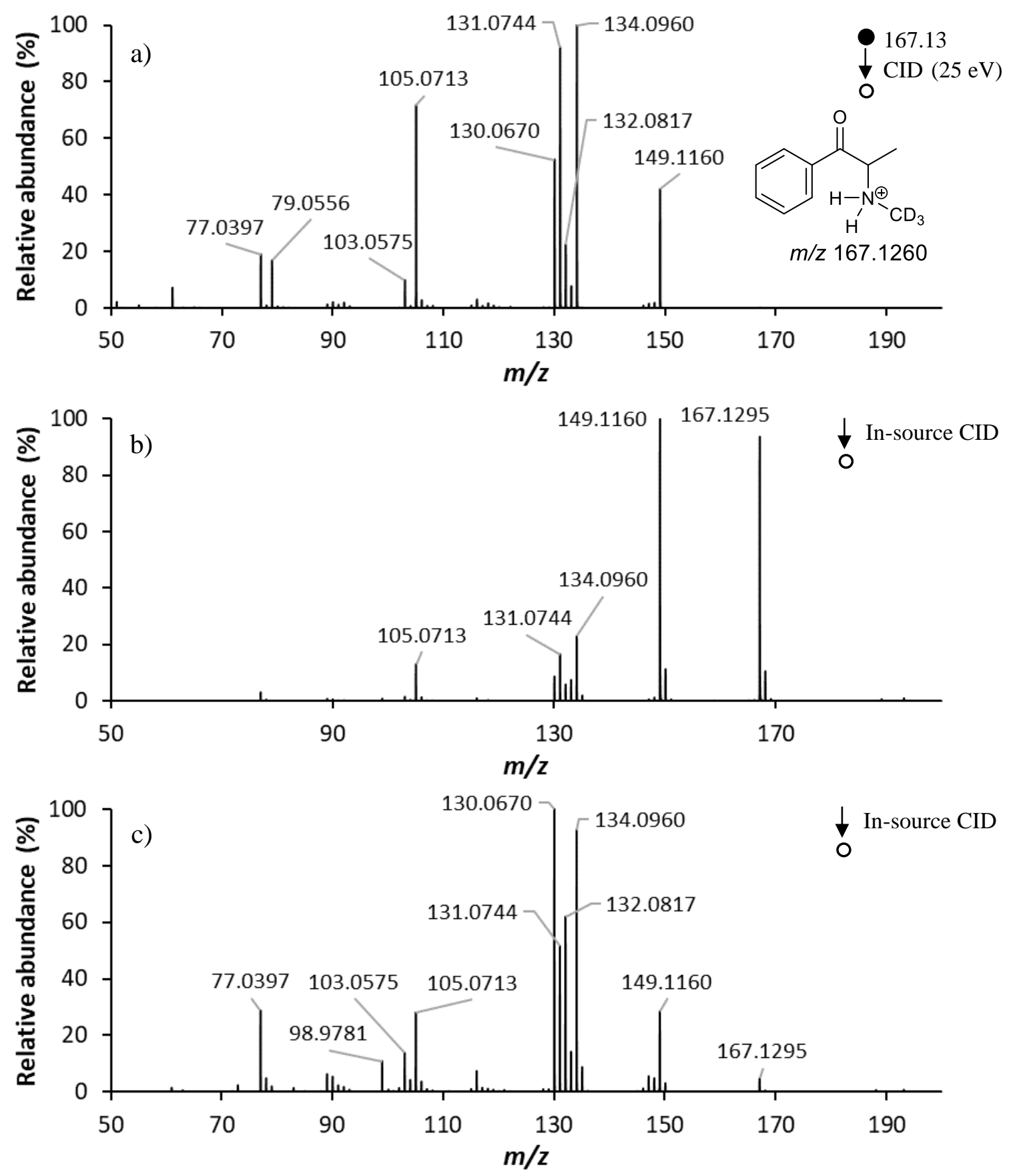

Figure 6.5. Comparison of beam-type CID (top) and in-source CID (middle and bottom) for protonated methcathinone- $\mathrm{d}_{3}$. For all spectra, the skimmer setting was held at $65 \mathrm{~V}$. For beamtype CID, the fragmentor setting was $175 \mathrm{~V}$ with a collision energy of $25 \mathrm{eV}$. For in-source CID, the fragmentor settings were $175 \mathrm{~V}$ and $255 \mathrm{~V}$, for b) and c), respectively with the collision energy set to $0 \mathrm{eV}$. 
Like methcathinone- $\mathrm{d}_{3}$, diethylpropion also has a relatively small molecular weight, but diethylpropion is a $3^{\circ}$ amine, $N$-alkylated, synthetic cathinone. Figure 6.6 provides a comparison between beam-type CID and in-source CID with the beam-type CID collected at a collision energy of $25 \mathrm{eV}$ and the in-source CID collected at a collision energy of $0 \mathrm{eV}$, a skimmer setting of $65 \mathrm{~V}$ and fragmentor settings of $225 \mathrm{~V}$ (Figure 6.6b) and $285 \mathrm{~V}$ (Figure 6.6c), respectively. The insource CID spectra reveal the conversion of the $[\mathrm{M}+\mathrm{H}]^{+}$protonated molecular ion at $\mathrm{m} / \mathrm{z} 206.1578$ for $\mathrm{C}_{13} \mathrm{H}_{20} \mathrm{NO}^{+}$(expected at $\mathrm{m} / 2,206.1544 ; 16$ ppm error) to product ions at $\mathrm{m} / \mathrm{z} 160.1157$ for $\mathrm{C}_{11} \mathrm{H}_{14} \mathrm{~N}^{+}$(expected at $\mathrm{m} / z \mathbf{z} 160.1126 ; 19$ ppm error), $\mathrm{m} / z, 133.0690$ for $\mathrm{C}_{9} \mathrm{H}_{9} \mathrm{O}^{+}$(expected at $\mathrm{m} / \mathrm{z}$ 133.0653; $28 \mathrm{ppm}$ error), $\mathrm{m} / \mathrm{z} 105.0713$ for $\mathrm{C}_{8} \mathrm{H}_{9}{ }^{+}$(expected at $\mathrm{m} / \mathrm{z}$ 105.0704; $9 \mathrm{ppm}$ error), $\mathrm{m} / \mathrm{z}$ 100.1164 for $\mathrm{C}_{6} \mathrm{H}_{14} \mathrm{~N}^{+}$(expected at $\mathrm{m} / 2$ 100.1126; 38 ppm error) and $m / 2.77 .0397$ for $\mathrm{C}_{6} \mathrm{H}_{5}{ }^{+}$ (expected at $\mathrm{m} / \mathrm{z} 77.0391 ; 8 \mathrm{ppm}$ error), and the most abundant product ions at $\mathrm{m} / \mathrm{z} 105.0713$ and $\mathrm{m} / \mathrm{z}, 100.1126$ are consistent with previous literature [155].

The product ions at $\mathrm{m} / \mathrm{z} 133.0690$ and $\mathrm{m} / z 100.1164$ are of particular importance because these product ions correspond with the formation of an alkylphenone and iminium cation, respectively. Our previous work with $N$-alkylated synthetic cathinones demonstrated that, whereas $2^{\circ}$ amines favor the loss of water, $3^{\circ}$ amines favor the formation of alkylphenones and a corresponding iminium counter ion [152]. The common phenylethyl ion at $\mathrm{m} / z .105 .0713$ and phenylium ion at $\mathrm{m} / \mathrm{z} 77.0397$ were also identified [34]. In general, the in-source CID product ions are in agreement with the beam-type CID product ions at a collision energy of $25 \mathrm{eV}$ (Figure 6.6a). One exception is the presence of the product ion at $m / z 72.0845, \mathrm{C}_{4} \mathrm{H}_{10} \mathrm{~N}^{+}$(expected at $m / z 72.0813 ; 44$ ppm error) in the beam-type CID spectrum, which arises through consecutive fragmentation from the intermediate at $\mathrm{m} / \mathrm{z}$ 100.1164. One explanation for the low abundance at $\mathrm{m} / \mathrm{z} 72$ in the in-source CID spectrum is that kinetic-based products are more competitive relative to this product of 
sequential neutral losses. Another reason for the absence of $m / z, 72$ in the in-source CID spectra could be due to instrumental discrimination against low mass ions at elevated fragmentor settings. In our experience, $m / z, 77$ was the lowest mass product ion of any significant abundance from dozens of in-source CID spectra, even though the scan range started at $\mathrm{m} / \mathrm{z} 50$ in all cases and the beam-type CID spectra often contained fragments between $\mathrm{m} / \mathrm{z}$ 50-77. 

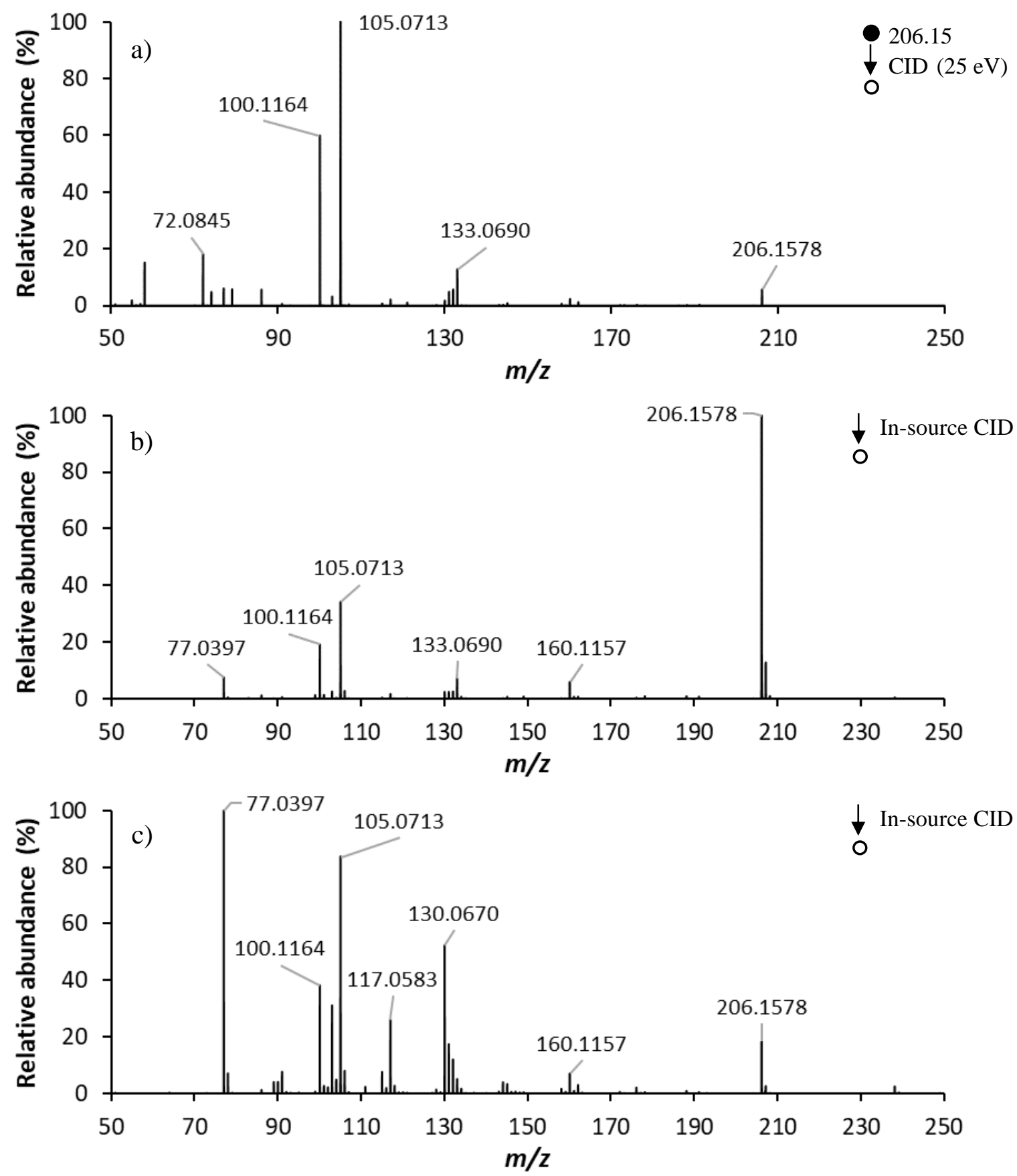

Figure 6.6. Comparison of beam-type CID (top) and in-source CID (middle and bottom) for protonated diethylpropion. For all spectra, the skimmer setting was held at $65 \mathrm{~V}$. For beam-type CID, the fragmentor setting was $225 \mathrm{~V}$ with a collision energy of $25 \mathrm{eV}$. For in-source CID, the fragmentor settings were $225 \mathrm{~V}$ and $285 \mathrm{~V}$, for b) and c), respectively with the collision energy set to $0 \mathrm{eV}$. 
Figure 6.7 shows the beam-type CID and in-source CID mass spectra for pentylone- $\mathrm{d}_{3}$, which is a relatively large molecular weight, $2^{\circ}$ amine, $N$-alkylated, synthetic cathinone. Figure 6.7c shows the in-source CID mass spectrum, which was collected with a collision energy of $0 \mathrm{eV}$, skimmer setting of $65 \mathrm{~V}$ and a fragmentor setting of $255 \mathrm{~V}$. Under these conditions, a significant portion of the $[\mathrm{M}+\mathrm{H}]^{+}$protonated precursor ion at $m / z, 239.1530$ for $\mathrm{C}_{13} \mathrm{H}_{15} \mathrm{D}_{3} \mathrm{NO}_{3}{ }^{+}$(expected at $\mathrm{m} / \mathrm{z}$ 239.1471; $25 \mathrm{ppm}$ error) is converted to product ions through collisional activation. The common product ions observed in Figure 6.7c include: $m / z 221.1395$ for $\mathrm{C}_{13} \mathrm{H}_{13} \mathrm{D}_{3} \mathrm{NO}_{2}{ }^{+}$(expected at $m / z$ 221.1366; 13 ppm error) $m / z, 191.1275$ for $\mathrm{C}_{12} \mathrm{H}_{11} \mathrm{D}_{3} \mathrm{NO}^{+}$(expected at $\mathrm{m} / z, 191.1260 ; 8 \mathrm{ppm}$ error), $m / z 178.0843$ for $\mathrm{C}_{10} \mathrm{H}_{6} \mathrm{D}_{3} \mathrm{NO}_{2}{ }^{+}$(expected at $m / z$ 178.0818; 14 ppm error), $m / z, 135.0465$ for $\mathrm{C}_{8} \mathrm{H}_{7} \mathrm{O}_{2}{ }^{+}$(expected at $m / z$ 135.0446; 14 ppm error) and $m / z, 89.1171$ for $\mathrm{C}_{5} \mathrm{H}_{9} \mathrm{D}_{3} \mathrm{~N}^{+}$(expected at $m / z$ 89.1154; 19 ppm error), among others.

The product ions at $m / z 221.1395$ and $m / z, 178.0843$ are formed through the loss of $\mathrm{H}_{2} \mathrm{O}$ and a propyl radical $\left({ }^{\circ} \mathrm{C}_{3} \mathrm{H}_{7}\right)$, respectively. As mentioned above, the radical losses from even-electron precursors are commonly observed in tandem mass spectra of $\mathrm{N}$-alkylated synthetic cathinones $[\underline{36}, \underline{37}, \underline{152}]$. Likewise, the product ion at $\mathrm{m} / z$ 191.1275 occurs through the loss of formaldehyde $\left(\mathrm{CH}_{2} \mathrm{O}\right)$, which is typical for methylenedioxy-containing synthetic cathinones $[\underline{15}, \underline{61}, \underline{75]}$. Finally, the product ions at $m / z 135.0465$ and $m / z 89.1171$ are consistent with the methylenedioxysubstituted tropylium ion and the deuterated iminium ion. As the collision energy is increased from $15 \mathrm{eV}$ in Figure 6.7a to $25 \mathrm{eV}$ in Figure 6.7b, the corresponding product ion spectrum shifts to lower mass ions through higher energy fragmentation events which allows for the generation of additional product ions, such as those at $\mathrm{m} / z 105.0359$ for $\mathrm{C}_{7} \mathrm{H}_{5} \mathrm{O}^{+}$(expected at $\mathrm{m} / \mathrm{z}$ 105.0340; 8 ppm error) and $m / z, 77.0397$ for $\mathrm{C}_{6} \mathrm{H}_{5}{ }^{+}$(expected at $m / z, 77.0391 ; 48$ ppm error). As with the fentanyl analogs, we cannot be sure whether the phenylium ion at $\mathrm{m} / \mathrm{z} 77.0397$ forms via direct 
cleavage or via consecutive cleavages. Whereas a potential pathway for the fentanyl analogs was via the loss of $\mathrm{C}_{2} \mathrm{H}_{2}$ from the phenylethyl ion at $m / \mathrm{z} 105.0713$, a potential intermediate for the cathinone analogs is via the loss of CO from the benzoyl ion at $m / z 105.0359$. 

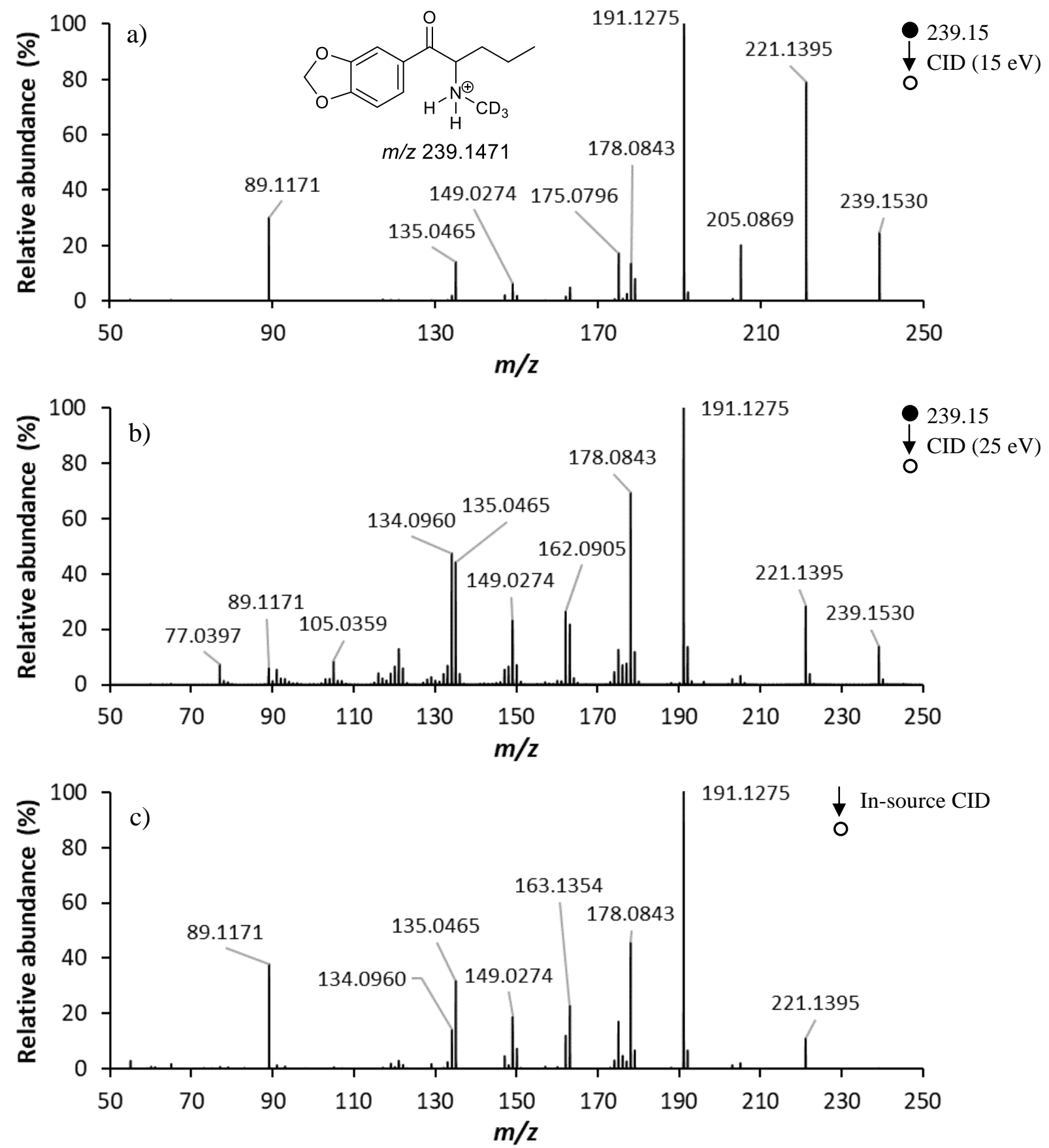

Figure 6.7. Comparison of beam-type CID (top and middle) and in-source CID (bottom) for protonated pentylone- $\mathrm{d}_{3}$. For all spectra, the skimmer setting was held at $65 \mathrm{~V}$. For beam-type $\mathrm{CID}$, the fragmentor setting was $175 \mathrm{~V}$ with collision energies of $15 \mathrm{eV}$ and $25 \mathrm{eV}$, for a) and b), respectively. For in-source CID, the fragmentor setting was $255 \mathrm{~V}$ with a collision energy of $0 \mathrm{eV}$. 
Dibutylone- $\mathrm{d}_{3}$ has the same molecular mass as pentylone- $\mathrm{d}_{3}(238 \mathrm{~g} / \mathrm{mol})$, but dibutylone- $\mathrm{d}_{3}$ is a $3^{\circ}$ amine, $N$-alkylated, synthetic cathinone. The in-source CID spectrum (Figure 6.8c), which was collected with a collision energy of $0 \mathrm{eV}$, a skimmer setting of $65 \mathrm{~V}$ and a fragmentor setting of $285 \mathrm{~V}$ reveals efficient conversion of the $[\mathrm{M}+\mathrm{H}]^{+}$protonated precursor at $\mathrm{m} / \mathrm{z} 239.1519$ $\left(\mathrm{C}_{13} \mathrm{H}_{15} \mathrm{D}_{3} \mathrm{NO}_{3}{ }^{+}\right.$expected at $\mathrm{m} / z 239.1471 ; 20 \mathrm{ppm}$ error $)$ to product ions. The product ions in Figure 6.8 include: $m / z 194.0920$ for $\mathrm{C}_{11} \mathrm{H}_{8} \mathrm{D}_{3} \mathrm{O}_{3}{ }^{+}$(expected at $\mathrm{m} / \mathrm{z} 194.0893 ; 14 \mathrm{ppm}$ error), $\mathrm{m} / \mathrm{z}$ 166.0993 for $\mathrm{C}_{10} \mathrm{H}_{8} \mathrm{D}_{3} \mathrm{O}_{2}{ }^{+}$(expected at $m / z$ 166.0944; 30 ppm error), $m / z 164.0822$ for $\mathrm{C}_{10} \mathrm{H}_{6} \mathrm{D}_{3} \mathrm{O}_{2}{ }^{+}$ (expected at $m / z$ 164.0787; 21 ppm error), $m / z 149.0262$ for $\mathrm{C}_{8} \mathrm{H}_{5} \mathrm{O}_{3}{ }^{+}$(expected at $\mathrm{m} / z$ 149.0238; $16 \mathrm{ppm}$ error), 136.0866 for $\mathrm{C}_{9} \mathrm{H}_{6} \mathrm{D}_{3} \mathrm{O}^{+}$(expected at $m / z, 136.0842 ; 17$ ppm error) 108.0929 for $\mathrm{C}_{8} \mathrm{H}_{6} \mathrm{D}_{3}{ }^{+}$(expected at $m / z$ 108.0889; 37 ppm error) and $m / z 89.1169$ for $\mathrm{C}_{5} \mathrm{H}_{9} \mathrm{D}_{3} \mathrm{~N}^{+}$(expected at $m / z$ 89.1154; 19 ppm error).

The product ion at $\mathrm{m} / \mathrm{z} 194.0920$ is formed through cleavage of the $N$-alkyl group, which is common for $3^{\circ}$ amine, $N$-alkylated synthetic cathinones [152]. The product ion at $\mathrm{m} / \mathrm{z} 164.0822$ results from the cleavage of formaldehyde $\left(\mathrm{CH}_{2} \mathrm{O}\right)$ from the intermediate at $\mathrm{m} / \mathrm{z}, 194.0920$, and the product ion at $\mathrm{m} / \mathrm{z} 149.0262$ is the methylenedioxy-substituted benzoylium ion. The loss of formaldehyde from methylenedioxy substituted cathinones has been reported previously for both $N$-alkylated synthetic cathinones and $\alpha$-pyrrolidinophenone synthetic cathinones $[\underline{15}, \underline{75}]$.

Using MS ${ }^{\mathrm{n}}$ on the LIT, the intermediates at $\mathrm{m} / \mathrm{z} 164.0822$ and 166.0944 can continue to lose small neutrals to form product ions at $m / z 136.0842$ and 108.0929, which are much more prominent in the higher energy beam-type CID spectrum and in-source CID than the lower energy beam-type CID spectrum. There are no mechanisms to form these lower mass fragments via direct cleavages, so their presence can only be explained by sequential neutral losses. The presence of the lowenergy product ion at $m / z 89.1169$, which corresponds to the deuterated iminium ion, provides 
further support for the presence of a synthetic cathinone. However, this low energy product ion is lowest in abundance in the in-source CID spectrum because other pathways and sequential neutral losses become more competitive. The in-source CID spectrum in Figure 6.8c contains greater differences in product ion abundances relative to the beam-type CID spectra at a collision energy of $15 \mathrm{eV}$ in Figure 6.8a and at $25 \mathrm{eV}$ in Figure 6.8b than the other spectral comparisons. Still, from the perspective of drug identification, the most abundant product ions in the beam-type CID spectra are among the most abundant product ions of the in-source CID spectra. 

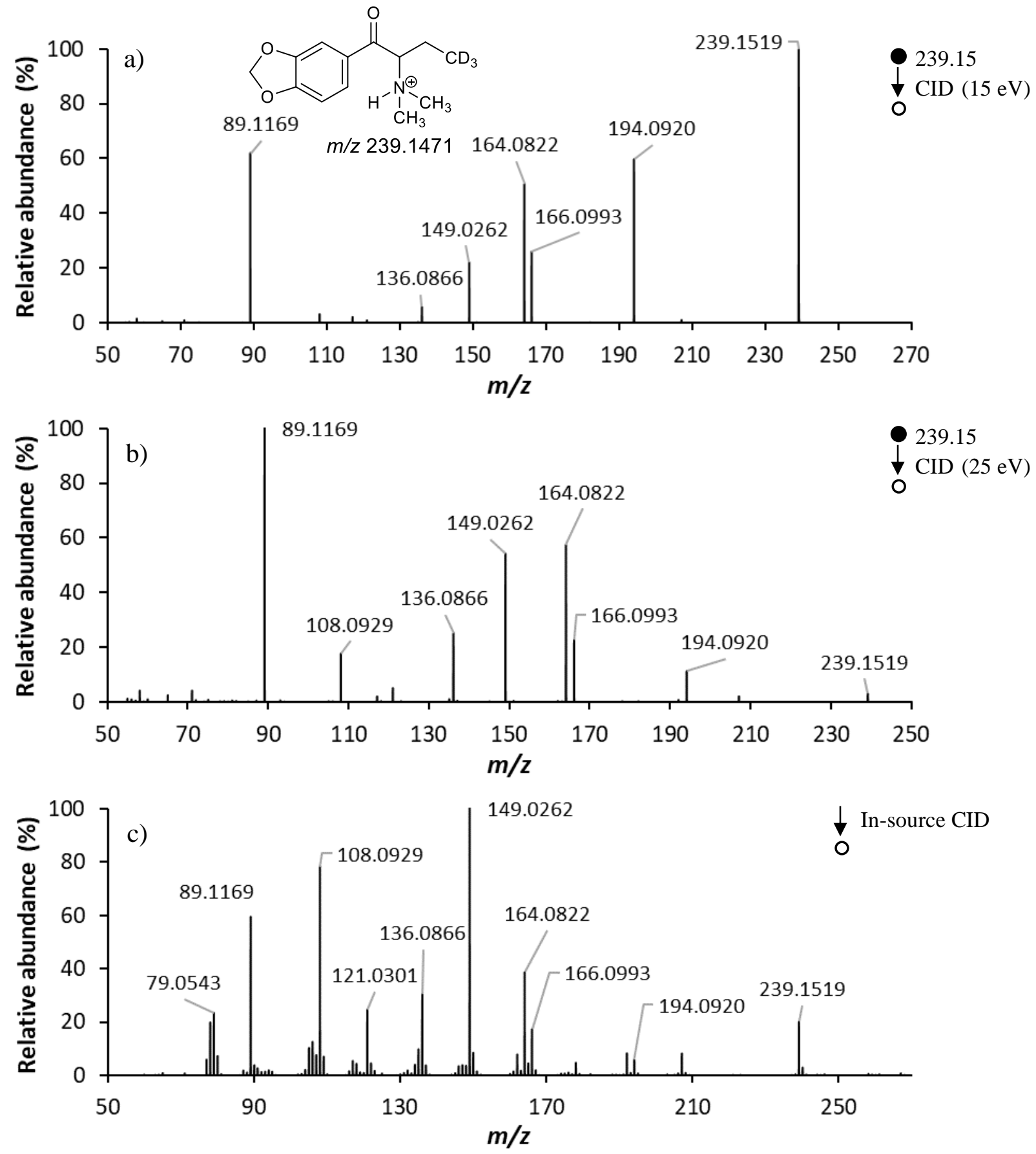

Figure 6.8. Comparison of beam-type CID (top and middle) and in-source CID (bottom) and) for protonated dibutylone- $\mathrm{d}_{3}$. For all spectra, the skimmer setting was held at $65 \mathrm{~V}$. For beamtype CID, the fragmentor setting was $225 \mathrm{~V}$ with collision energies of $15 \mathrm{eV}$ and $25 \mathrm{eV}$, for a) and b), respectively. For in-source CID, the fragmentor settings was $285 \mathrm{~V}$ with a collision energy of $0 \mathrm{eV}$. 


\subsubsection{Discussion of Synthetic Cathinones}

Through the analysis of a series of $N$-alkylated synthetic cathinones, we have identified the general consistency between product ion spectra of in-source CID and beam-type CID on the same instrument with the same ion source. The compounds analyzed had a range of molecular weights and various degrees of substitution, including the presence of $2^{\circ}$ and $3^{\circ}$ amines, which are known to favor different pathways during tandem mass spectrometry [152]. Whereas in-source CID and beam-type CID spectra are generally similar in the distribution and types of product ions formed, the spectra can be distinguished based on subtle differences in relative ion abundances and by the fact that the in-source CID spectra typically provide increased noise and additional adducts relative to beam-type CID spectra. Differences between in-source CID spectra and beam-type CID spectra can be ascribed to four major factors: 1) in-source CID spectra can include ions from different precursor molecules; 2) in-source CID spectra can contain product ions from different adducts of the same precursor molecules; 3) in-source CID spectra appear to access kinetically favored, higher-energy fragmentation pathways; and 4) in-source CID spectra seem to suffer from discrimination against low-mass product ions in the region $\mathrm{m} / \mathrm{z}$ 50-77.

As examples of factors 1 and 2 above, the high energy in-source CID spectrum of methcathinone- $\mathrm{d}_{3}(255 \mathrm{~V}$, Figure 6.5c) shows an abundant product ion (peak drop-in) at $\mathrm{m} / \mathrm{z}$ 98.9781, which is absent from the lower energy in-source CID spectrum (175 V, Figure 6.5b) and the beam-type CID spectrum (Figure 6.5a). We assume that this fragment derives from a sulfuric acid impurity or from a precursor with a higher activation energy than methcathinone- $\mathrm{d}_{3}$. Supporting this hypothesis, the same product ion at $\mathrm{m} / \mathrm{z} 98.9781$ was observed in other in-source CID spectra, such as ortho-methylfentanyl (Figure 6.1b). The impurity could derive from a contaminant within the solvent, the residual gases, the sample container or the ionization source itself (i.e. PEEK tubing). An example of peak drop-out for the in-source CID spectra is the product 
ion at $\mathrm{m} / z 79.0556$ for $\mathrm{C}_{6} \mathrm{H}_{7}+$ (expected at $\mathrm{m} / z, 79.0547 ; 11 \mathrm{ppm}$ error) in Figure 6.5a. This product ion is only present in the beam-type CID. The occurence of ion drop-in/drop-out is particularly tricky when dealing with more complex samples or matrices than the drug standards used in this study.

The nature of in-source CID implies that the product ions generated through in-source CID arise not only from the analyte of interest but also from any compound present in the source during the in-source CID process. Practically, this means that the product ions present in the in-source CID product ion spectra are not exclusively derived from analyte of interest. This fundamental principle limits the applicability of in-source CID for the structural elucidation of unknown compounds; however, in-source CID has demonstrated moderate success with the differentiation of structurally similar compounds such as synthetic cathinones $[32,156]$, synthetic cannabinoids [145] and fentanyl analogs [119]. One approach to determine which product ions in an in-source CID spectrum derive from which precursor ions in a mixture is to use chemometrics [157]. In the absence of more-extensive validation, beam-type isolation and fragmentation will continue to be required for potential unequivocal differentiation of isomers and isobars [158].

Two approaches that have been applied in an attempt to overcome the downfalls of in-source CID are the combination of chromatography and the generation of instrument specific in-source CID mass spectral libraries [130]. Chromatography allows for the separation of the analyte of interest from other potential interferences; however, chromatography does not address sourcespecific contamination and co-elution. The application of in-source CID mass spectral libraries can be manufacturer specific - to account for the effects of source design — and, to have the highest power of discrimination, instrument specific [151]. When feasible, the generation of composite mass spectra through summed or averaged mass spectra collected at different potentials has shown 
the capability to identify unknown compounds based on in-house generated in-source CID mass spectral libraries [130].

\subsection{Conclusions}

The analysis of a series of previously characterized fentanyl analogs [106] and synthetic cathinones [152] with a Q-TOF mass spectrometer allowed for a qualitative assessment of the similarities and differences in the product ion spectra generated with in-source CID and beam-type CID conditions. In this study, we demonstrate that, under certain conditions, it is possible to generate visually similar product ion spectra between in-source CID and beam-type CID of the same substance. However, although in-source CID and beam-type CID both encourage consecutive neutral losses at elevated collision energies, both techniques tend to produce kinetically favored fragments at lower masses relative to trapping-type CID. Of the three techniques, in-source CID seems to access the highest energy pathways and tends to show the greatest extent of peak drop-in/drop-out and elevated noise from contaminant ions. The subtle differences in relative ion abundances between in-source CID and beam-type CID can usually be explained by the preference for higher energy pathways in in-source CID, especially at elevated fragmentor voltages. In some cases, the abundance of peaks that are separated by only a few Daltons, such as $m / z 165$ and $m / z 170$ for alfentanil, can show ratios as disparate as 0.75:1 for trapping-type CID to 1.25:1 for beam-type CID and 5:1 for in-source CID. 


\section{Conclusions and Future Work}

\section{Synthetic Cathinones}

This dissertation combined the use of multi-stage mass spectrometry $\left(\mathrm{MS}^{\mathrm{n}}\right)$, accurate mass measurements with high-resolution mass spectrometry (HRMS), isotopic labeling, density functional theory (DFT) calculations and gas-phase ion spectroscopy for the structural characterization of synthetic cathinones and FRCs. Through this work we have identified conserved fragmentation pathways for synthetic cathinones and FRCs, proposed mechanisms for the formation of characteristic ions through protonated tandem mass spectrometry (MS/MS) and electron ionization mass spectrometry (EI-MS) and provided examples of how to apply our now broadened understanding of the fragmentation behavior of these two classes of emerging synthetic drugs to the identification of novel synthetic cathinones and FRCs.

The identification of the complex gas-phase rearrangements that occur for the formation of the tropylium ion $(\mathrm{m} / \mathrm{z}, 91)$ or substituted derivative ions from the $\alpha$-pyrrolidinophenone class of synthetic cathinones is a significant contribution to our understanding of the protonated MS/MS fragmentation behavior of synthetic cathinones. Throughout literature the tropylium ion $(\mathrm{m} / \mathrm{z}, 91)$ or substituted derivative ions are observed among the most abundant peaks in protonated MS/MS spectra; however, prior to this work there has never been an explanation for this experimental observation that was supported with the use of $\mathrm{MS}^{\mathrm{n}}$, HRMS, isotopic labeling, DFT calculations and ion spectroscopy. The identification of different oxygen containing intermediates that likely contain a phthalane-like core structure and the almost exclusive retention of the $\alpha$-carbon in the tropylium ion has never been reported before in literature. Likewise, the identification of the competitive pathways for the loss of $\mathrm{CO}$ and ethylene $\left(\mathrm{C}_{2} \mathrm{H}_{4}\right)$ from a primary intermediate ion provides support for the direct loss of CO from the alkyl side chain. 
A continuation of the tropylium ion project involved the analysis of $22 \alpha$-pyrrolidinophenone synthetic cathinones using three different ionization and fragmentation techniques commonly used for forensic toxicology, seized drug analysis and research at national laboratories. The identification of conserved tandem mass spectrometry fragmentation pathways through the loss of $\mathrm{CH}_{2} \mathrm{C}_{\mathrm{n}} \mathrm{H}_{2 \mathrm{n}}, \mathrm{H}_{2} \mathrm{O},{ }^{\cdot} \mathrm{C}_{2} \mathrm{H}_{4} \mathrm{C}_{\mathrm{n}} \mathrm{H}_{\mathrm{n} 2+1}, \mathrm{C}_{6} \mathrm{H}_{6}$, and $\mathrm{NC}_{4} \mathrm{H}_{9}$ from protonated molecular ions provides a series of diagnostic ions that can be used for the MS/MS identification of $\alpha$-pyrrolidinophenone synthetic cathinones. Other identified fragmentation pathways are through the formation of iminium ions and the formation of tropylium and phenylethyl ions at $m / z, 91\left(\mathrm{C}_{7} \mathrm{H}_{7}{ }^{+}\right)$and $m / z, 105\left(\mathrm{C}_{8} \mathrm{H}_{9}{ }^{+}\right)$. These pathways should assist practitioners with determining whether or not novel emerging synthetic drugs belong to the class of $\alpha$-pyrrolidinophenone synthetic cathinones, and if so, the nature of their structure.

Another major class of synthetic cathinones that was extensively characterized through this work is the $N$-alkylated class of synthetic cathinones. The use of GC-EI-MS, ESI-MS/MS, and DART-MS/MS enabled characteristic protonated MS/MS fragmentation pathways and mechanistic origins for the observed product ions. Specifically, diagnostic ions through the loss of $\mathrm{H}_{2} \mathrm{O}, \mathrm{C}_{\mathrm{n}} \mathrm{H}_{2 \mathrm{n}+3} \mathrm{~N}$, and $\mathrm{C}_{7} \mathrm{H}_{6} \mathrm{O}$ for $2^{\circ}$ amines and the favored formation of iminium ions and alkylphenones for $3^{\circ}$ amines. Likewise, the demonstration of the loss of alkyl radicals $\left({ }^{\circ} \mathrm{C}_{\mathrm{n}} \mathrm{H}_{2 \mathrm{n}+1}\right)$ from the amine and aliphatic chain and the loss of alkenes $\left(\mathrm{C}_{\mathrm{n}} \mathrm{H}_{2 n}\right)$ from the amine moiety for all $N$-alkylated synthetic cathinones helps with the identification of future $N$-alkylated synthetic cathinones. Finally, the development of proposed mechanistic explanations for the observed protonated MS/MS and EI-MS fragmentation and examples of how to apply this knowledge to the identification of novel $\mathrm{N}$-alkylated synthetic cathinones provides useful resources for forensic toxicologists and seized drug analysts. 
The application of our now-broadened understanding of the protonated MS/MS and EI-MS fragmentation behavior for $\alpha$-pyrrolidinophenone and $N$-alkylated synthetic cathinones will help analysts to better understand and defend their observations and interpretations in existing and future casework. The development of characteristic fragmentation pathways for $\alpha$ pyrrolidinophenone and $N$-alkylated synthetic cathinones provides a template for the identification of novel synthetic cathinones through conserved fragmentation behavior. Likewise, the location of substitution for novel synthetic cathinones can be identified through divergence from the provided characteristic fragmentation pathways or the presence of characteristic product ions, such as the phenylethyl ion $\left(\mathrm{C}_{8} \mathrm{H}_{9}{ }^{+}\right)$at $\mathrm{m} / 2105$ for aromatic substituted synthetic cathinones. These manuscripts can be used as a series of guides or references for seized drug analysts and toxicologists in their pursuit of the identification of novel synthetic cathinones that arise in casework, similar to the work of Zuba for EI-MS [15].

The demonstration that ESI and DART ionization sources on the same Q-TOF mass spectrometer produced even-electron protonated molecular ions and highly similar MS/MS spectra through the analysis of 40 synthetic cathinones further supports expansion of the use of ambient ionization sources. Likewise, the identification of the highly conserved fragmentation pathways between the LIT and Q-TOF mass spectrometers provides a measure of robustness of these characteristic fragmentation pathways. However, it is important to recognize that the multicollisional environment of the LIT tends to limit the extent of consecutive fragmentation relative to the Q-TOF [35]. The Q-TOF therefore favors the formation of lower mass ions relative to the LIT. Finally, knowledge about the systematic tendencies of high energy radical-driven fragmentation in EI-MS and lower energy collisional activation of protonated precursor ions in MS/MS can be used to help support the identification of emerging synthetic cathinones. 


\section{Future Work Following the Study on Synthetic Cathinones}

Future areas of research based on the synthetic cathinone portion of this work could include an expansion of this work from synthetic cathinones to other classes of emerging synthetic drugs, such as synthetic cannabinoids and benzodiazepines. Synthetic cannabinoids are a large class of emerging synthetic drugs that show promise for the identification of complex gas-phase rearrangements due to the presence of multiple basic sites and aromatic regions. In contrast, the diazepine functionality of the benzodiazepines class of emerging synthetic drugs could potentially support complex gas-phase rearrangements based on the presence of two nucleophilic nitrogen atoms in the core structure. Another example of a complex gas-phase rearrangement was introduced in Chapter 4 of this work, through the proposed R-group transfer for fentanyl, which represents the synthetic opioid class of emerging synthetic drugs.

\section{Fentanyl Analogs}

The second major portion of this work focused on the structural characterization of fentanyl and FRCs. The primary investigation revealed the identification of three isobaric fentanyl product ions at $\mathrm{m} / \mathrm{z} 188$ including a novel product ion formed through the intermediate product ion at $\mathrm{m} / \mathrm{z}$ 216. The identification of three isobaric fentanyl product ions at $m / z 188$ has a potential impact on the product ion selection for quantitative analyses because these isobaric product ions have different rates and energies of formation. Throughout literature, the product ion at $\mathrm{m} / \mathrm{z} 188$ is commonly reported as a product ion used for quantification based on monitoring the transition from $m / z \quad 337 \rightarrow m / z$ 188. However, differences in rates and energies of formation can lead to variation in ion abundances, which can affect the accuracy and precision of quantitative analyses [97]. From the point-of-view of structural characterization, the recognition of these different fragmentation pathways can help identify similar mechanisms in emerging FRCs. As new FRCs enter the drug market our ability to identify characteristic fragmentation pathways and conserved 
fragmentation mechanisms can assist medical examiners, toxicologists and seized drug analysts with the identification of novel FRCs.

In an extension of Chapter 4, FRCs substituted at five common regions of substitution to the core fentanyl structure were analyzed to assess the influence of chemical modification to the core fentanyl structure on the observed protonated product ion spectra. The regions of substitution included the aniline ring, amide moiety, piperidine ring, $N$-alkyl chain, and cyclic substituent, which is typically a phenyl, thiol or tetrazole. This work provides an approach, based on the observed product ions from ESI-MS/MS, to identify the modification site(s) on the core fentanyl structure for FRCs. The determination that substitutions to the aniline ring and amide moiety result in remarkably conserved fragmentation pathways, whereas substitutions to the piperidine ring, $\mathrm{N}$ alkyl chain, and cyclic substituent result in distinct differences in fragmentation pathways, is a significant contribution from this study. Likewise, the relatively conserved nature of the five most abundant product ions between the LIT and Q-TOF instruments provides guidance to the forensic community about how to identify the location of substitution for FRCs that is applicable across different MS platforms. Finally, the identification of the conserved fragmentation pathways, when accounting for differences in the mass and location of the substituent for FRCs, provides an additional tool for the identification of novel FRCs to toxicologists and seized drug analysts.

The final chapter of this dissertation involved a comparison between the MS/MS spectra generated from in-source CID and beam-type CID, where in-source CID involves fragmentation in the source region of the mass spectrometer as compared to isolation and fragmentation in the collision cell for beam-type CID. The application of in-source CID for single-stage HRMS instrumentation has grown in popularity within the forensic community because of the desire to obtain both molecular weight and structural information through pseudo-MS/MS analysis. This 
chapter demonstrated that, under certain conditions, it is possible to generate visually similar product ion spectra between in-source CID and beam-type CID experiments. However, ion dropin and drop-out was observed for some of the comparisons, which highlights the need for caution when analyzing potential mixtures or complex biological samples where strict control of precursor ions present in the source region may not be possible. When combined with chromatography or ion mobility, and with the use of in-house generated mass spectral libraries, in-source CID has potential to become a reliable mainstream technique not just for screening, but for confirmatory identifications of seized drugs [130].

The identification of characteristic fragmentation pathways and the mechanistic origins of the observed product ions for fentanyl and FRCs is a significant contribution from this work. Likewise, the identification of the influence of chemical modification to the core fentanyl structure on the observed product ion spectra provides a valuable tool to seized drug analysts and toxicologists for the identification of emerging FRCs. Our now-broadened understanding of the propensity for certain chemical modifications to direct fragmentation down particular pathways will assist practitioners with the identification of novel FRCs. However, research within the area of FRC modifications will need to continue to further develop our understanding of the effect of modification on the fragmentation behavior of FRCs as the list of emerging FRCs continues to grow.

\section{Future Work Following the Study on Fentanyl Analogs}

One direction for future research with FRC modification is the development of an MS/MS mass spectral search algorithm that is capable of identifying emerging FRCs. The algorithm could make use of both absolute peak positions and the positions of peaks relative to the neutral losses from the precursor ion. In fact, an equivalent technique has already been developed for EI-MS data; it is called the Hybrid Similarity Search (HSS), and it was recently developed by the Mass 
Spectrometry Data Center at NIST [92]. The HSS algorithm shifts neutral loss peaks in the library spectrum to match the corresponding neutral loss peaks in the query spectrum and equally weights neutral loss and direct fragment matches, which allows for the identification of structurally similar compounds. However, the HSS algorithm struggles with the identification of structurally similar compounds that alter the fragmentation mechanism or contain more than one substitution from the closest compound in the reference library.

To develop a similar algorithm for tandem mass spectra of protonated FRCs, one would have to examine the effects trap-, beam- and in-source CID and the effects of the applied collision energy on the MS/MS mass spectral search algorithm. The end goal would be to provide seized drug analysts and toxicologists with a MS/MS mass spectral search algorithm embedded directly in the instrument software that would aid with the identification of known and novel FRCs.

Overall, the impact of this work will be determined by the willingness of seized drug analysts and forensic toxicologists to use these resources as guidelines for the identification of emerging synthetic cathinones and FRCs. The identification of conserved fragmentation pathways for synthetic cathinones and FRCs, as well as the development of proposed mechanisms for the formation of characteristic ions through protonated MS/MS and EI-MS helps broaden our current understanding of these two classes of emerging synthetic drugs. Likewise, providing an explanation for the formation of the tropylium ion $(\mathrm{m} / \mathrm{z}, 91)$ for protonated synthetic cathinones and the identification of three isobaric fentanyl product ions at $\mathrm{m} / \mathrm{z} 188$ will help analysts better understand and defend their observations and interpretations and potentially influence product ions selection for quantification with FRCs. Finally, an assessment of the impact of chemical modifications to the core fentanyl structure on the observed product ion spectra and a comparison between in-source CID and beam-type CID provides practitioners with a methodology for the 
determination of modification sites for FRCs and offers a word of caution for the use of in-source CID for structural elucidation. In conclusion, this work has provided seized drug analysts and toxicologists with additional tools to combat the ever-growing challenge of emerging synthetic drug identification. 


\section{References}

[1] M.J. Valente, P. Guedes de Pinho, M. de Lourdes Bastos, F. Carvalho, M. Carvalho, Khat and synthetic cathinones: a review, Arch. Toxicol. 88 (1) (2014) 15-45. DOI: 10.1007/s00204-013-1163-9.

[2] K. Zaitsu, M. Katagi, M. Tatsuno, T. Sato, H. Tsuchihashi, K. Suzuki, Recently abused $\beta$-keto derivatives of 3,4-methylenedioxyphenylalkylamines: a review of their metabolisms and toxicological analysis, Forensic Toxicol. 29 (2) (2011) 73-84. DOI: 10.1007/s11419-011-0111-8.

[3] A.L. Bretteville-Jensen, S.S. Tuv, O.R. Bilgrei, B. Field, L. Bachs, Synthetic Cannabinoids and Cathinones: Prevalence and Markets, Forensic Sci. Rev. 25 (2013) 726.

[4] J.A. Fass, A.D. Fass, A.S. Garcia, Synthetic cathinones (bath salts): legal status and patterns of abuse, Ann. Pharmacother. 46 (3) (2012) 436-441. DOI: 10.1345/aph.1Q628.

[5] P. Griffiths, D. Lopez, R. Sedefov, A. Gallegos, B. Hughes, A. Noor, L. Royuela, Khat use and monitoring drug use in Europe: the current situation and issues for the future, $J$. Ethnopharmacol. 132 (3) (2010) 578-583. DOI: 10.1016/j.jep.2010.04.046.

[6] E.E. Balint, G. Falkay, G.A. Balint, Khat - a controversial plant, Wien. Klin. Wochenschr. 121 (2009) 604-614. DOI: 10.1007/s00508-009-1259-7.

[7] J.P. Kelly, Cathinone derivatives: a review of their chemistry, pharmacology and toxicology, Drug Test. Anal. 3 (2011) 439-453. DOI: 10.1002/dta.313.

[8] M. Coppola, R. Mondola, Synthetic cathinones: chemistry, pharmacology and toxicology of a new class of designer drugs of abuse marketed as "bath salts" or "plant food", Toxicol. Lett. 211 (2) (2012) 144-149. DOI: 10.1016/j.toxlet.2012.03.009.

[9] P.I. Dargan, R. Sedefov, A. Gallegos, D.M. Wood, The pharmacology and toxicology of the synthetic cathinone mephedrone (4-methylmethcathinone), Drug Test. Anal. 3 (7-8) (2011) 454-463. DOI: 10.1002/dta.312.

[10] J. Jerry, G. Collins, D. Streem, Synthetic legal intoxicating drugs: the emerging 'incense' and 'bath salt' phenomenon, Cleve. Clin. J. Med. 79 (4) (2012) 258-264. DOI: 10.3949/ccjm.79a.11147.

[11] T.A. Dal Carson, R. Young, R.A. Glennon, Cathinone: An Investigation of Several NAlkyl and Methylenedioxy-Substituted Analogs., Pharmacol. Biochem. Behav. 58 (4) (1997) 1109-1116.

[12] F. Westphal, T. Junge, P. Rosner, G. Fritschi, B. Klein, U. Girreser, Mass spectral and NMR spectral data of two new designer drugs with an alpha-aminophenone structure: 4'methyl-alpha-pyrrolidinohexanophenone and 4'-methyl-alpha-pyrrolidinobutyrophenone, Forensic Sci. Int. 169 (2007) 32-42. DOI: 10.1016/j.forsciint.2006.07.024.

[13] A. Namera, M. Kawamura, A. Nakamoto, T. Saito, M. Nagao, Comprehensive review of the detection methods for synthetic cannabinoids and cathinones, Forensic Toxicol. 33 (2) (2015) 175-194. DOI: 10.1007/s11419-015-0270-0.

[14] Y. Abiedalla, K. Abdel-Hay, J. DeRuiter, C.R. Clark, Differentiation of cyclic tertiary amine cathinone derivatives by product ion electron ionization mass spectrometry, Rapid Commun. Mass Spectrom. 30 (6) (2016) 763-772. DOI: 10.1002/rcm.7491.

[15] D. Zuba, Identification of cathinones and other active components of 'legal highs' by mass spectrometric methods, Trends Anal. Chem. 32 (2012) 15-30. DOI: 10.1016/j.trac.2011.09.009. 
[16] E. Fornal, Identification of substituted cathinones: 3,4-Methylenedioxy derivatives by high performance liquid chromatography-quadrupole time of flight mass spectrometry, $J$. Pharm. Biomed. Anal. 81-82 (2013) 13-19. DOI: 10.1016/j.jpba.2013.03.016.

[17] P. Jankovics, A. Varadi, L. Tolgyesi, S. Lohner, J. Nemeth-Palotas, H. Koszegi-Szalai, Identification and characterization of the new designer drug 4'-methylethcathinone (4MEC) and elaboration of a novel liquid chromatography-tandem mass spectrometry (LCMS/MS) screening method for seven different methcathinone analogs, Forensic Sci. Int. 210 (2011) 213-220. DOI: 10.1016/j.forsciint.2011.03.019.

[18] S. Matsuta, N. Shima, H. Kamata, H. Kakehashi, S. Nakano, K. Sasaki, T. Kamata, H. Nishioka, A. Miki, M. Katagi, K. Zaitsu, T. Sato, H. Tsuchihashi, K. Suzuki, Metabolism of the designer drug alpha-pyrrolidinobutiophenone (alpha-PBP) in humans:

identification and quantification of the phase I metabolites in urine, Forensic Sci. Int. 249 (2015) 181-188. DOI: 10.1016/j.forsciint.2015.02.004.

[19] C. Sauer, F.T. Peters, C. Haas, M.R. Meyer, G. Fritschi, H.H. Maurer, New designer drug alpha-pyrrolidinovalerophenone (PVP): studies on its metabolism and toxicological detection in rat urine using gas chromatographic/mass spectrometric techniques, J. Mass Spectrom. 44 (6) (2009) 952-964. DOI: 10.1002/jms.1571.

[20] Y. Abiedalla, J. DeRuiter, C.R. Clark, GC-MS, GC-MS/MS and GC-IR differentiation of carbonyl modified analogues of MDPV, Forens. Chem. 3 (2017) 58-68. DOI: 10.1016/j.forc.2016.11.002.

[21] K. Hasegawa, O. Suzuki, A. Wurita, K. Minakata, I. Yamagishi, H. Nozawa, K. Gonmori, K. Watanabe, Postmortem distribution of $\alpha$-pyrrolidinovalerophenone and its metabolite in body fluids and solid tissues in a fatal poisoning case measured by LCMS-MS with the standard addition method, Forensic Toxicol. 32 (2) (2014) 225-234. DOI: 10.1007/s11419-014-0227-8.

[22] B. Waters, N. Ikematsu, K. Hara, H. Fujii, T. Tokuyasu, M. Takayama, A. Matsusue, M. Kashiwagi, S. Kubo, GC-PCI-MS/MS and LC-ESI-MS/MS databases for the detection of 104 psychotropic compounds (synthetic cannabinoids, synthetic cathinones, phenethylamine derivatives), Leg. Med. 20 (2016) 1-7. DOI: 10.1016/j.legalmed.2016.02.006.

[23] M. Concheiro, S. Anizan, K. Ellefsen, M.A. Huestis, Simultaneous quantification of 28 synthetic cathinones and metabolites in urine by liquid chromatography-high resolution mass spectrometry, Anal. Bioanal. Chem. 405 (29) (2013) 9437-9448. DOI: 10.1007/s00216-013-7386-z.

[24] M. Paillet-Loilier, A. Cesbron, R. Le Boisselier, J. Bourgine, D. Debruyne, Emerging drugs of abuse: current perspectives on substituted cathinones, Subst. Abuse Rehabil. 5 (2014) 37-52. DOI: 10.2147/SAR.S37257.

[25] D. Ammann, J.M. McLaren, D. Gerostamoulos, J. Beyer, Detection and quantification of new designer drugs in human blood: Part 2 - Designer cathinones, J. Anal. Toxicol. 36 (6) (2012) 381-389. DOI: 10.1093/jat/bks049.

[26] M.J. Swortwood, K.N. Ellefsen, A. Wohlfarth, X. Diao, M. Concheiro-Guisan, R. Kronstrand, M.A. Huestis, First metabolic profile of PV8, a novel synthetic cathinone, in human hepatocytes and urine by high-resolution mass spectrometry, Anal. Bioanal. Chem. 408 (18) (2016) 4845-4856. DOI: 10.1007/s00216-016-9599-4.

[27] M. Ibanez, O.J. Pozo, J.V. Sancho, T. Orengo, G. Haro, F. Hernandez, Analytical strategy to investigate 3,4-methylenedioxypyrovalerone (MDPV) metabolites in 
consumers' urine by high-resolution mass spectrometry, Anal. Bioanal. Chem. 408 (1) (2016) 151-164. DOI: 10.1007/s00216-015-9088-1.

[28] O.J. Pozo, M. Ibanez, J.V. Sancho, J. Lahoz-Beneytez, M. Farre, E. Papaseit, R. de la Torre, F. Hernandez, Mass spectrometric evaluation of mephedrone in vivo human metabolism: identification of phase I and phase II metabolites, including a novel succinyl conjugate, Drug Metab. Dispos. 43 (2) (2015) 248-257. DOI: 10.1124/dmd.114.061416.

[29] D. Fabregat-Safont, X. Carbón, C. Gil, M. Ventura, J.V. Sancho, F. Hernández, M. Ibáñez, Reporting the novel synthetic cathinone 5-PPDI through its analytical characterization by mass spectrometry and nuclear magnetic resonance, Forensic Toxicol. 36 (2) (2018) 447-457. DOI: 10.1007/s11419-018-0422-0.

[30] Z. Qian, W. Jia, T. Li, Z. Hua, C. Liu, Identification of five pyrrolidinyl substituted cathinones and the collision-induced dissociation of electrospray-generated pyrrolidinyl substituted cathinones, Drug Test. Anal. 9 (5) (2017) 778-787. DOI: 10.1002/dta.2035.

[31] L. Bijlsma, J.V. Sancho, F. Hernandez, W.M. Niessen, Fragmentation pathways of drugs of abuse and their metabolites based on QTOF MS/MS and $\mathrm{MS}^{\mathrm{E}}$ Eaccurate-mass spectra, J. Mass Spectrom. 46 (9) (2011) 865-875. DOI: 10.1002/jms.1963.

[32] J.D. Power, S.D. McDermott, B. Talbot, J.E. O'Brien, P. Kavanagh, The analysis of amphetamine-like cathinone derivatives using positive electrospray ionization with insource collision-induced dissociation, Rapid Commun. Mass Spectrom. 26 (22) (2012) 2601-2611. DOI: 10.1002/rcm.6383.

[33] J. Martens, G. Berden, C.R. Gebhardt, J. Oomens, Infrared ion spectroscopy in a modified quadrupole ion trap mass spectrometer at the FELIX free electron laser laboratory, Rev. Sci. Instrum. 87 (10) (2016) 103108. DOI: 10.1063/1.4964703.

[34] Frisch MJ, Trucks GW, Schlegel HB, et al. Gaussian 09, Revision A.02.Wallingford CT: Gaussian, Inc; 2016.

[35] L. Sleno, D. Volmer, Ion activation methods for tandem mass spectrometry, J. Mass Spectrom. 39 (2004) 1091-1112.

[36] E. Fornal, Formation of odd-electron product ions in collision-induced fragmentation of electrospray-generated protonated cathinone derivatives: aryl alpha-primary amino ketones, Rapid Commun. Mass Spectrom. 27 (16) (2013) 1858-1866. DOI: 10.1002/rcm.6635.

[37] E. Fornal, Study of collision-induced dissociation of electrospray-generated protonated cathinones, Drug Test. Anal. 6 (2014) 705-715. DOI: 10.1002/dta.1573.

[38] P.N. Rylander, S. Meyerson, H.M. Grubb, Organic Ions in the Gas Phase. II. The Tropylium Ion, J. Am. Chem. Soc. 79 (4) (1956) 842-846.

[39] F.W. McLafferty, J. Winkler, Gaseous Tropylium, Benzyl, Tolyl, and Norbornadienyl Cations, J. Am. Chem. Soc. 96 (16) (1974) 5182-5189.

[40] F.W. McLafferty, F.M. Bockhoff, Formation of Benzyl and Tropylium Ions from Gaseous Toluene and Cycloheptatriene Cations, J. Am. Chem. Soc. 101 (7) (1979) 17831786.

[41] C. Lifshitz, Tropylium Ion Formation from Toluene: Solution of an Old Problem in Organic Mass Spectrometry, Acc. Chem. Res. 27 (1994) 138-144.

[42] M.J. Hayward, F.D.S. Park, L.M. Phelan, S.L. Bernasek, Ä. Somogyi, V.H. Wysocki, Examination of Sputtered Ion Mechanisms Leading to the Formation of $\mathrm{C}_{7} \mathrm{H}_{7}, J . A m$. Chem. Soc. 118 (35) (1996) 8375-8380. 
[43] M. C. Cone, J.S. Dewar, D. Landman, Gaseous Ions. 1. MINDO/3 Study of the Rearrangement of Benzyl Cation to Tropylium, J. Am. Chem. Soc. 99 (2) (1977).

[44] M.J.S. Dewar, D. Landman, Gaseous Ions. 2.1 MINDO/3 Study of the Rearrangements of Toluene and Cycloheptatriene Molecular Ions and the Formation of Tropylium, J. Am. Chem. Soc. 99 (8) (1977).

[45] B.J. Smith, N.E. Hall, G2(MP2,SVP) study of the relationship between the benzyl and tropyl radicals, and their cation analogues, Chem. Phys. Lett. 279 (1997) 165-171.

[46] I.S. Ignatyev, T. Sundius, Competitive ring hydride shifts and tolyl-benzyl rearrangements in tolyl and silatolyl cations, Chem. Phys. Lett. 326 (2000) 101-108.

[47] K.W. Bullins, T.T.S. Huang, S.J. Kirkby, Theoretical investigation of the formation of the tropylium ion from the toluene radical cation, Int. J. Quantum Chem. 109 (6) (2009) 1322-1327. DOI: 10.1002/qua.21956.

[48] T.D. Fridgen, J. Troe, A.A. Viggiano, A.J. Midey, S. Williams, T.B. McMahon, Experimental and Theoretical Studies of the Benzylium+/Tropylium+ Ratios after Charge Transfer to Ethylbenzene, J. Phys. Chem. A. 108 (2004) 5600-5609.

[49] M. Vala, J. Oomens, G. Berden, Structure and Dissociation Pathways of Protonated Tetralin (1,2,3,4-Tetrahydronaphthalene), J. Phys. Chem. A. 121 (24) (2017) 4606-4612. DOI: $10.1021 /$ acs.jpca.7b01858.

[50] A. Wurita, K. Hasegawa, K. Minakata, K. Gonmori, H. Nozawa, I. Yamagishi, O. Suzuki, K. Watanabe, Postmortem distribution of alpha-pyrrolidinobutiophenone in body fluids and solid tissues of a human cadaver, Leg. Med. 16 (5) (2014) 241-246. DOI: 10.1016/j.legalmed.2014.05.001.

[51] S.A. McLuckey, D.E. Goeringer, Slow Heating Methods in Tandem Mass Spectrometry, Int. J. Mass Spectrom. 32 (1997) 461-474.

[52] J. Mitchell Wells, S.A. McLuckey, Collision-Induced Dissociation (CID) of Peptides and Proteins, in Methods in Enzymology. 2005. pp. 148-185.

[53] B.L. Murray, C.M. Murphy, M.C. Beuhler, Death following recreational use of designer drug "bath salts" containing 3,4-Methylenedioxypyrovalerone (MDPV), J. Med. Toxicol. 8 (1) (2012) 69-75. DOI: 10.1007/s13181-011-0196-9.

[54] K.N. Ellefsen, M. Concheiro, M.A. Huestis, Synthetic cathinone pharmacokinetics, analytical methods, and toxicological findings from human performance and postmortem cases, Drug Metab. Rev. 48 (2) (2016) 237-265. DOI: 10.1080/03602532.2016.1188937.

[55] Drug Enforcement Administration, Department of Justice, Schedules of Controlled Substances: Temporary Placement of Three Synthetic Cathinones Into Schedule I, Fed. Regist. 76 (174) (2011).

[56] K. Zaitsu, M. Katagi, H. Tsuchihashi, A. Ishii, Recently abused synthetic cathinones, $\alpha-$ pyrrolidinophenone derivatives: a review of their pharmacology, acute toxicity, and metabolism, Forensic Toxicol. 32 (1) (2014) 1-8. DOI: 10.1007/s11419-013-0218-1.

[57] F. Westphal, T. Junge, B. Klein, G. Fritschi, U. Girreser, Spectroscopic characterization of 3,4-methylenedioxypyrrolidinobutyrophenone: a new designer drug with alphapyrrolidinophenone structure, Forensic Sci. Int. 209 (2011) 126-132. DOI: 10.1016/j.forsciint.2011.01.016.

[58] L.D. Simmler, T.A. Buser, M. Donzelli, Y. Schramm, L.H. Dieu, J. Huwyler, S. Chaboz, M.C. Hoener, M.E. Liechti, Pharmacological characterization of designer cathinones in vitro, Br. J. Pharmacol. 168 (2) (2013) 458-470. DOI: 10.1111/j.1476-

5381.2012.02145.x. 
[59] M. Coppola, R. Mondola, 3,4-methylenedioxypyrovalerone (MDPV): chemistry, pharmacology and toxicology of a new designer drug of abuse marketed online, Toxicol. Lett. 208 (1) (2012) 12-15. DOI: 10.1016/j.toxlet.2011.10.002.

[60] S. Matsuta, M. Katagi, H. Nishioka, H. Kamata, K. Sasaki, N. Shima, T. Kamata, Miki. A., M. Tatsuno, K. Zaitsu, K. Tsuboi, H. Tsuchihashi, K. Suzuki, Structural characterization of cathinone-type designer drugs by EI mass spectrometry, Jpn. J. Forensic Sci. Tech. . 19 (2) (2014) 77-89.

[61] J.T. Davidson, E.L. Piacentino, Z.J. Sasiene, Y. Abiedalla, J. DeRuiter, C.R. Clark, G. Berden, J. Oomens, V. Ryzhov, G.P. Jackson, Identification of novel fragmentation pathways and fragment ion structures in the tandem mass spectra of protonated synthetic cathinones, Forens. Chem. 19 (2020). DOI: 10.1016/j.forc.2020.100245.

[62] A. H. Beckett, G. R. Jones, D.A. Hollingsbee, Degradation of (-)-ephedrine in solution and during extraction with diethyl ether, J. Pharm. Pharmac. 30 (1978) 15-19.

[63] J. DeRuiter, L. Hays, A. Valaer, C.R. Clark, Methcathinone and Designer Drug Analogs: Synthesis, Stereochemical Analysis, and Analytical Properties, J. Chromatogr. Sci. 32 (1994) 552-564.

[64] S. Kerrigan, M. Savage, C. Cavazos, P. Bella, Thermal Degradation of Synthetic Cathinones: Implications for Forensic Toxicology, J. Anal. Toxicol. 40 (1) (2016) 1-11. DOI: $10.1093 /$ jat/bkv099.

[65] F. Westphal, T. Junge, P. Rosner, F. Sonnichsen, F. Schuster, Mass and NMR spectroscopic characterization of 3,4-methylenedioxypyrovalerone: a designer drug with alpha-pyrrolidinophenone structure, Forensic Sci. Int. 190 (2009) 1-8. DOI: 10.1016/j.forsciint.2009.05.001.

[66] A.D. Krikorian, Khat and its use: an historical perspective, J. Ethnopharmacol. 12 (2) (1984) 115-178.

[67] L.L. Ioannides-Demos, L. Piccenna, J.J. McNeil, Pharmacotherapies for obesity: past, current, and future therapies, J. Obes. 2011 (2011) 1-18. DOI: 10.1155/2011/179674.

[68] M.G. Bossong, J.P. Van Dijk, R.J. Niesink, Methylone and mCPP, two new drugs of abuse?, Addict. Biol. 10 (4) (2005) 321-323. DOI: 10.1080/13556210500350794.

[69] Schedules of Controlled Substances; Placement of Methcathinone into Schedule I, Fed. Regist. 58 (198) (1993) 53404-53406.

[70] M. Majchrzak, R. Celinski, P. Kus, T. Kowalska, M. Sajewicz, The newest cathinone derivatives as designer drugs: an analytical and toxicological review, Forensic Toxicol. 36 (1) (2018) 33-50. DOI: 10.1007/s11419-017-0385-6.

[71] L. Bijlsma, J.V. Sancho, F. Hernandez, W.M. Niessen, Fragmentation pathways of drugs of abuse and their metabolites based on QTOF MS/MS and MS(E) accurate-mass spectra, J. Mass Spectrom. 46 (9) (2011) 865-875. DOI: 10.1002/jms.1963.

[72] J. Martinez-Clemente, R. Lopez-Arnau, M. Carbo, D. Pubill, J. Camarasa, E. Escubedo, Mephedrone pharmacokinetics after intravenous and oral administration in rats: relation to pharmacodynamics, Psychopharmacology. 229 (2) (2013) 295-306. DOI: 10.1007/s00213-013-3108-7.

[73] F.W. McLafferty, Mass Spectrometric Analysis Molecular Rearrangements, Anal. Chem. 31 (1) (1959) 82-87.

[74] M. Cydzik, M. Rudowska, P. Stefanowicz, Z. Szewczuk, The competition of charge remote and charge directed fragmentation mechanisms in quaternary ammonium salt 
derivatized peptides--an isotopic exchange study, J. Am. Soc. Mass Spectrom. 22 (12) (2011) 2103-2107. DOI: 10.1007/s 13361-011-0245-2.

[75] J.T. Davidson, Z.J. Sasiene, Y. Abiedalla, J. DeRuiter, C.R. Clark, G.P. Jackson, Fragmentation pathways of $\alpha$-pyrrolidinophenone synthetic cathinones and their application to the identification of emerging synthetic cathinone derivatives, Int. J. Mass Spectrom. Accepted for publication (2020). DOI: 10.1016/j.ijms.2020.116343.

[76] R.B. Cody, Why Are We Still Reporting Mass Accuracy in Parts per Million (ppm)?, J. Am. Soc. Mass Spectrom. 31 (4) (2020) 1004-1005. DOI: 10.1021/jasms.9b00150.

[77] M. Majchrzak, R. Celinski, T. Kowalska, M. Sajewicz, Fatal case of poisoning with a new cathinone derivative: alpha-propylaminopentiophenone (N-PP), Forensic Toxicol. 36 (2) (2018) 525-533. DOI: 10.1007/s11419-018-0417-x.

[78] Wilfried M. A. Niessen, R.A. Correa, Interpretation of MS-MS Mass Spectra of Drugs and Pesticides: Chapter 4 Fragmentation of Drugs and Pesticides. 2017: John Wiley \& Sons. pp. 241-247.

[79] M.P. Levitas, E. Andrews, I. Lurie, I. Marginean, Discrimination of synthetic cathinones by GC-MS and GC-MS/MS using cold electron ionization, Forensic Sci. Int. 288 (2018) 107-114. DOI: 10.1016/j.forsciint.2018.04.026.

[80] H.E. Schueler, Emerging Synthetic Fentanyl Analogs, Acad. Forens. Path. 7 (1) (2017) 36-40. DOI: 10.23907/2017.004.

[81] Drugs of Abuse A DEA Resource Guide. 2017, DEA: U.S. Department of Justice Drug Enforcement Administration. pp. 40-41.

[82] P. Armenian, K.T. Vo, J. Barr-Walker, K.L. Lynch, Fentanyl, fentanyl analogs and novel synthetic opioids: A comprehensive review, Neuropharmacology. 134 (2018) 121-132. DOI: 10.1016/j.neuropharm.2017.10.016.

[83] NFLIS-Drug 2017 Annual Report. 2017, U.S. Department of Justice Drug Enforcement Administration: National Forensic Laboratory Information System (NFLIS).

[84] Control of Immediate Precursor Used in the Illicit Manufacture of Fentanyl as a Schedule II Controlled Substance, Fed. Regist. 75 (124) (2010).

[85] J. Suzuki, S. El-Haddad, A review: Fentanyl and non-pharmaceutical fentanyls, Drug Alcohol Depend. 171 (2017) 107-116. DOI: 10.1016/j.drugalcdep.2016.11.033.

[86] Recommended methods for the Identification and Analysis of Fentanyl and its Analogues in Biological Specimens. 2017: United Nations Office on Drugs and Crime. pp. 5-11.

[87] I.S. Lurie, R. Iio, Use of multiple-reaction monitoring ratios for identifying incompletely resolved fentanyl homologs and analogs via ultra-high-pressure liquid chromatographytandem mass spectrometry, J. Chromatogr. A. 1216 (9) (2009) 1515-1519. DOI: 10.1016/j.chroma.2008.12.097.

[88] J.R. Mallette, J.F. Casale, S.G. Toske, P.A. Hays, Characterization of (2R,4S)- and (2R,4R)-2-Methylfentanyl and their differentiation from cis- and trans-3-Methylfentanyl, Forens. Chem. 8 (2018) 64-71. DOI: 10.1016/j.forc.2018.02.001.

[89] J.R. Mallette, J.F. Casale, P.A. Hays, Characterization and Differentiation of Cyclopropylfentanyl from E-Crotonylfentanyl, Z-Crotonylfentanyl, and 3Butenylfentanyl, Sci. Justice. 59 (1) (2018) 67-74.

[90] T. Kanamori, Y.T. Iwata, H. Segawa, T. Yamamuro, K. Kuwayama, K. Tsujikawa, H. Inoue, Characterization and Differentiation of Geometric Isomers of 3-methylfentanyl Analogs by Gas Chromatography/Mass Spectrometry, Liquid Chromatography/Mass 
Spectrometry, and Nuclear Magnetic Resonance Spectroscopy, J. Forensic. Sci. 62 (6) (2017) 1472-1478. DOI: 10.1111/1556-4029.13395.

[91] H. Ohta, Suzuki, S., and Ogasawara, K., Studies on Fentanyl and Related Compounds IV. Chromatographic and Spectrometric Discrimination of Fentanyl and its Derivatives, $J$. Anal. Toxicol. 23 (1999) 280-285.

[92] A.S. Moorthy, W.E. Wallace, A.J. Kearsley, D.V. Tchekhovskoi, S.E. Stein, Combining Fragment-Ion and Neutral-Loss Matching during Mass Spectral Library Searching: A New General Purpose Algorithm Applicable to Illicit Drug Identification, Anal. Chem. 89 (24) (2017) 13261-13268. DOI: 10.1021/acs.analchem.7b03320.

[93] K.E. Strayer, H.M. Antonides, M.P. Juhascik, R. Daniulaityte, I.E. Sizemore, LCMS/MS-Based Method for the Multiplex Detection of 24 Fentanyl Analogues and Metabolites in Whole Blood at Sub ng mL $\mathrm{mL}^{-1}$ Concentrations, ACS Omega. 3 (1) (2018) 514-523. DOI: 10.1021/acsomega.7b01536.

[94] M. Thevis, H. Geyer, D. Bahr, W. Schanzer, Identification of fentanyl, alfentanil, sufentanil, remifentanil and their major metabolites in human urine by liquid chromatography/tandem mass spectrometry for doping control purposes, Eur. J. Mass Spectrom. 11 (4) (2005) 419-427. DOI: 10.1255/ejms.761.

[95] M.G. Feasel, A. Wohlfarth, J.M. Nilles, S. Pang, R.L. Kristovich, M.A. Huestis, Metabolism of Carfentanil, an Ultra-Potent Opioid, in Human Liver Microsomes and Human Hepatocytes by High-Resolution Mass Spectrometry, AAPS J. 18 (6) (2016) 1489-1499. DOI: 10.1208/s12248-016-9963-5.

[96] A.T. Caspar, A.B. Kollas, H.H. Maurer, M.R. Meyer, Development of a quantitative approach in blood plasma for low-dosed hallucinogens and opioids using LC-high resolution mass spectrometry, Talanta. 176 (2018) 635-645. DOI: 10.1016/j.talanta.2017.08.063.

[97] W. Wichitnithad, T.J. McManus, P.S. Callery, Identification of isobaric product ions in electrospray ionization mass spectra of fentanyl using multistage mass spectrometry and deuterium labeling, Rapid Commun. Mass Spectrom. 24 (17) (2010) 2547-2553. DOI: 10.1002/rcm.4673.

[98] E.N. Shoff, M.E. Zaney, J.H. Kahl, G.W. Hime, D.M. Boland, Qualitative Identification of Fentanyl Analogs and Other Opioids in Postmortem Cases by UHPLC-Ion Trap-MS J. Anal. Toxicol. 41 (6) (2017) 484-492. DOI: 10.1093/jat/bkx041.

[99] J. Rittgen, M. Putz, R. Zimmermann, Identification of fentanyl derivatives at trace levels with nonaqueous capillary electrophoresis-electrospray-tandem mass spectrometry $\left(\mathrm{MS}^{\mathrm{n}}\right.$, $\mathrm{n}=2,3)$ : analytical method and forensic applications, Electrophoresis. 33 (11) (2012) 1595-1605. DOI: 10.1002/elps.201100655.

[100] T. Breindahl, A. Kimergard, M.F. Andreasen, D.S. Pedersen, Identification of a new psychoactive substance in seized material: the synthetic opioid N-phenyl-N-[1-(2phenethyl)piperidin-4-yl]prop-2-enamide (Acrylfentanyl), Drug Test Anal. 9 (3) (2017) 415-422. DOI: 10.1002/dta.2046.

[101] C. Noble, P. Weihe Dalsgaard, S. Stybe Johansen, K. Linnet, Application of a screening method for fentanyl and its analogues using UHPLC-QTOF-MS with data-independent acquisition (DIA) in $\mathrm{MS}^{\mathrm{E}}$ mode and retrospective analysis of authentic forensic blood samples, Drug Test Anal. 10 (4) (2018) 651-662. DOI: 10.1002/dta.2263. 
[102] D.E. Koch, R. Isaza, J.W. Carpenter, R.P. Hunter, Simultaneous extraction and quantitation of fentanyl and norfentanyl from primate plasma with LC/MS detection, $J$. Pharm. Biomed. Anal. 34 (3) (2004) 577-584. DOI: 10.1016/s0731-7085(03)00652-6.

[103] K. Pihlainen, K. Grigoras, S. Franssila, R. Ketola, T. Kotiaho, R. Kostiainen, Analysis of amphetamines and fentanyls by atmospheric pressure desorption/ionization on silicon mass spectrometry and matrix-assisted laser desorption/ionization mass spectrometry and its application to forensic analysis of drug seizures, J. Mass Spectrom. 40 (4) (2005) 539545. DOI: $10.1002 / \mathrm{jms} .831$.

[104] Y. Takashina, T. Naito, Y. Mino, Y. Kagawa, J. Kawakami, Validated LC coupled to ESI-MS/MS analysis for fentanyl in human plasma and UV analysis in applied reservoir transdermal patches using a simple and rapid procedure, J. Clin. Pharm. Ther. 34 (5) (2009) 523-529. DOI: 10.1111/j.1365-2710.2009.01033.x.

[105] T. Grzeskowiak, A. Zgola-Grzeskowiak, D. Rusinska-Roszak, I. ZaporowskaStachowiak, M. Jeszka-Skowron, Fragmentation studies of selected drugs utilized in palliative care, Eur. J. Mass Spectrom. (2018) 420-436. DOI: $10.1177 / 1469066718812459$.

[106] J.T. Davidson, Z.J. Sasiene, G.P. Jackson, The influence of chemical modifications on the fragmentation behavior of fentanyl and fentanyl-related compounds in electrospray ionization tandem mass spectrometry, Drug Test. Anal. Accepted for Publication (2020). DOI: $10.1002 /$ dta.2794.

[107] P. Armenian, K.T. Vo, J. Barr-Walker, K.L. Lynch, Fentanyl, fentanyl analogs and novel synthetic opioids: A comprehensive review, Neuropharmacology. 134 (Pt A) (2018) 121132. DOI: 10.1016/j.neuropharm.2017.10.016.

[108] H.E. Schueler, Emerging Synthetic Fentanyl Analogs, Academic Forensic Pathology. 7 (1) (2017) 36-40. DOI: 10.23907/2017.004.

[109] P.G. Rose, M.S. Macfee, M.V. Boswell, Fentanyl Transdermal System Overdose Secondary to Cutaneous Hyperthermia, Anesth. Analg. 77 (1993) 390-391.

[110] Drug Enforcement Administration, Department of Justice, Schedules of Controlled Substances: Temporary Placement of FentanylRelated Substances in Schedule I, Fed. Regist. 83 (25) (2018) 5188-5192.

[111] NFLIS-Drug 2018 Annual Report. 2018, U.S. Department of Justice Drug Enforcement Administration: National Forensic Laboratory Information System (NFLIS).

[112] H. Ohta, S. Suzuki, K. Ogasawara, Studies on Fentanyl and Related Compounds IV. Chromatographic and Spectrometric Discrimination of Fentanyl and its Derivatives, $J$. Anal. Toxicol. 23 (1999) 280-285.

[113] T. Kanamori, Y.T. Iwata, H. Segawa, T. Yamamuro, K. Kuwayama, K. Tsujikawa, H. Inoue, Characterization and Differentiation of Geometric Isomers of 3-methylfentanyl Analogs by Gas Chromatography/Mass Spectrometry, Liquid Chromatography/Mass Spectrometry, and Nuclear Magnetic Resonance Spectroscopy, J. Forensic Sci. 62 (6) (2017) 1472-1478. DOI: 10.1111/1556-4029.13395.

[114] J.R. Mallette, J.F. Casale, S.G. Toske, P.A. Hays, Characterization of ( 2R,4S )- and ( 2R,4R )-2-Methylfentanyl and their differentiation from cis - and trans -3Methylfentanyl, Forens. Chem. 8 (2018) 64-71. DOI: 10.1016/j.forc.2018.02.001.

[115] J.R. Mallette, J.F. Casale, P.A. Hays, Characterization and Differentiation of Cyclopropylfentanyl from E-Crotonylfentanyl, Z-Crotonylfentanyl, and 3Butenylfentanyl, Sci. Justice. 59 (1) (2019) 67-74. 
[116] J.V. Abonamah, B.A. Eckenrode, M. Moini, On-site detection of fentanyl and its derivatives by field portable nano-liquid chromatography-electron lonization-mass spectrometry (nLC-EI-MS), Forens. Chem. 16 (2019). DOI: 10.1016/j.forc.2019.100180.

[117] K.E. Strayer, H.M. Antonides, M.P. Juhascik, R. Daniulaityte, I.E. Sizemore, LCMS/MS-Based Method for the Multiplex Detection of 24 Fentanyl Analogues and Metabolites in Whole Blood at Sub ng mL(-1) Concentrations, J. Am. Chem. Soc. 3 (1) (2018) 514-523. DOI: 10.1021/acsomega.7b01536.

[118] C.R. Ferreira, K.E. Yannell, A.K. Jarmusch, V. Pirro, Z. Ouyang, R.G. Cooks, Ambient Ionization Mass Spectrometry for Point-of-Care Diagnostics and Other Clinical Measurements, Clin. Chem. 62 (1) (2016) 99-110. DOI: 10.1373/clinchem.2014.237164.

[119] E. Sisco, J. Verkouteren, J. Staymates, J. Lawrence, Rapid detection of fentanyl, fentanyl analogues, and opioids for on-site or laboratory based drug seizure screening using thermal desorption DART-MS and ion mobility spectrometry, Forens. Chem. 4 (2017) 108-115. DOI: 10.1016/j.forc.2017.04.001.

[120] A. Moore, J. Foss, M. Juhascik, S. Botch-Jones, F. Kero, Rapid screening of opioids in seized street drugs using ambient ionization high resolution time-of-flight mass spectrometry, Forens. Chem. 13 (2019). DOI: 10.1016/j.forc.2019.100149.

[121] J.T. Davidson, Z.J. Sasiene, G.P. Jackson, The characterization of isobaric product ions of fentanyl using multi-stage mass spectrometry, high-resolution mass spectrometry and isotopic labeling, Drug Test. Anal. 12 (4) (2020) 496-503. DOI: 10.1002/dta.2758.

[122] Q. Nan, W. Hejian, X. Ping, S. Baohua, Z. Junbo, D. Hongxiao, Q. Huosheng, S. Fenyun, S. Yan, Investigation of Fragmentation Pathways of Fentanyl Analogues and Novel Synthetic Opioids by Electron Ionization High-Resolution Mass Spectrometry and Electrospray Ionization High-Resolution Tandem Mass Spectrometry, J. Am. Soc. Mass Spectrom. 31 (2) (2020) 277-291. DOI: 10.1021/jasms.9b00112.

[123] S.A. McLuckey, Principles of Collisional Activation in Analytical Mass Spectrometry, $J$. Am. Soc. Mass Spectrom. . 192 (3) (1992) 599-614.

[124] T.B. McMahon, Thermochemical ladders: Scaling the ramparts of gaseous ion energetics, Int. J. Mass Spectrom. 200 (2000) 187-199.

[125] L. Sleno, D.A. Volmer, Ion activation methods for tandem mass spectrometry, J. Mass Spectrom. 39 (10) (2004) 1091-1112. DOI: 10.1002/jms.703.

[126] S.A. McLuckey, D.E. Goeringer, Slow Heating Methods in Tandem Mass Spectrometry, J. Mass Spectrom. 32 (1997) 461-474.

[127] J. Mitchell Wells, S.A. McLuckey, Collision-Induced Dissociation (CID) of Peptides and Proteins, in Methods in Enzymology, Ed. A.L. Burlingame, Editor. 2005. pp. 148185.

[128] F.W. McLafferty, D.A. Stauffer, S.Y. Loh, C. Wesdemiotis, Unknown Identification Using Reference Mass Spectra. Quality Evaluation of Databases, J. Am. Soc. Mass Spectrom. 10 (1999) 1229-1240.

[129] W. Weinmann, A. Wiedemann, B. Eppinger, M. Renz, M. Svoboda, Screening for Drugs in Serum by Electrospray Ionization/Collision-Induced Dissociation and Library Searching, J. Am. Soc. Mass Spectrom. 10 (1999) 1028-1037.

[130] J.L. Josephs, Characterization of Over-the-counter Cough/Cold Medications by Liquid Chromatography /Electrospray Mass Spectrometry, Rapid. Commun. Mass Spectrom. 9 (1995) 1270-1274. 
[131] V. Katta, S.K. Chowdhury, B.T. Chait, Use of a Single-Quadrupole Mass Spectrometer for Collision-Induced Dissociation Studies of Multiply Charged Peptide Ions Produced by Electrospray Ionization, Anal. Chem. 63 (2) (1991) 174-178. DOI: 10.1021/ac00002a016,.

[132] S. Purvine, J.T. Eppel, E.C. Yi, D.R. Goodlett, Shotgun collision-induced dissociation of peptides using a time of flight mass analyzer, Proteomics. 3 (6) (2003) 847-850. DOI: 10.1002/pmic.200300362.

[133] G.J. Van Berkel, S.A. McLuckey, G.L. Glish, Electrospray Ionization of Porphyrins Using a Quadrupole Ion Trap for Mass Analysis, Anal. Chem. 63 (11) (1991) 1098-1109.

[134] J.A. Loo, H.R. Udseth, R.D. Smith, Collisional Effects on the Charge Distribution o Ions from Large Molecules, Formed by Electrospray-ionization Mass Spectrometry, Rapid. Commun. Mass Spectrom. 2 (10) (1988) 207-210.

[135] R.R. Steiner, R.L. Larson, Validation of the direct analysis in real time source for use in forensic drug screening, J. Forensic. Sci. 54 (3) (2009) 617-622. DOI: 10.1111/j.15564029.2009.01006.x.

[136] A.D. Lesiak, R.A. Musah, R.B. Cody, M.A. Domin, A.J. Dane, J.R. Shepard, Direct analysis in real time mass spectrometry (DART-MS) of "bath salt" cathinone drug mixtures, Analyst. 138 (12) (2013) 3424-3432. DOI: 10.1039/c3an00360d.

[137] W. Weinmann, M. Stoertzel, S. Vogt, J. Wendt, Tune compounds for electrospray ionisation /in-source collision-induced dissociation with mass spectral library searching,

J. Chromatogr. A. 926 (2001) 199-209.

[138] S. Gwak, J.R. Almirall, Rapid screening of 35 new psychoactive substances by ion mobility spectrometry (IMS) and direct analysis in real time (DART) coupled to quadrupole time-of-flight mass spectrometry (QTOF-MS), Drug. Test. Anal. 7 (10) (2015) 884-893. DOI: 10.1002/dta.1783.

[139] M.J. Pavlovich, B. Musselman, A.B. Hall, Direct analysis in real time-Mass spectrometry (DART-MS) in forensic and security applications, Mass Spectrom. Rev. 37 (2) (2018) 171-187. DOI: 10.1002/mas.21509.

[140] R.B. Cody, J.A. Laremée, H.D. Durst, Versatile New Ion Source for the Analysis of Materials in Open Air under Ambient Conditions, Anal. Chem. 77 (8) (2005) 2297-2302.

[141] J.H. Gross, Direct analysis in real time--a critical review on DART-MS, Anal. Bioanal. Chem. 406 (1) (2014) 63-80. DOI: 10.1007/s00216-013-7316-0.

[142] R.A. Musah, R.B. Cody, M.A. Domin, A.D. Lesiak, A.J. Dane, J.R. Shepard, DART-MS in-source collision induced dissociation and high mass accuracy for new psychoactive substance determinations, Forensic. Sci. Int. 244 (2014) 42-49. DOI: 10.1016/j.forsciint.2014.07.028.

[143] K.L. Fowble, J.R.E. Shepard, R.A. Musah, Identification and classification of cathinone unknowns by statistical analysis processing of direct analysis in real time-high resolution mass spectrometry-derived "neutral loss" spectra, Talanta. 179 (2018) 546-553. DOI: 10.1016/j.talanta.2017.11.020.

[144] E. Sisco, T.P. Forbes, M.E. Staymates, G. Gillen, Rapid Analysis of Trace Drugs and Metabolites Using a Thermal Desorption DART-MS Configuration, Anal. Methods. 8 (35) (2016) 6494-6499. DOI: 10.1039/C6AY01851C.

[145] R.A. Musah, M.A. Domin, R.B. Cody, A.D. Lesiak, A.J. Dane, J.R. Shepard, Direct analysis in real time mass spectrometry with collision-induced dissociation for structural 
analysis of synthetic cannabinoids, Rapid. Commun. Mass Spectrom. 26 (19) (2012) 2335-2342. DOI: 10.1002/rcm.6354.

[146] A.D. Lesiak, K.J. Adams, M.A. Domin, C. Henck, J.R. Shepard, DART-MS for rapid, preliminary screening of urine for DMAA, Drug. Test. Anal. 6 (7-8) (2014) 788-796. DOI: $10.1002 /$ dta. 1540.

[147] A.D. Lesiak, R.B. Cody, M. Ubukata, R.A. Musah, Direct analysis in real time high resolution mass spectrometry as a tool for rapid characterization of mind-altering plant materials and revelation of supplement adulteration--The case of Kanna, Forensic. Sci. Int. 260 (2016) 66-73. DOI: 10.1016/j.forsciint.2015.12.037.

[148] A.D. Lesiak, R.A. Musah, Application of ambient ionization high resolution mass spectrometry to determination of the botanical provenance of the constituents of psychoactive drug mixtures, Forensic. Sci. Int. 266 (2016) 271-280. DOI: 10.1016/j.forsciint.2016.06.009.

[149] L. Abranko, J.F. Garcia-Reyes, A. Molina-Diaz, In-source fragmentation and accurate mass analysis of multiclass flavonoid conjugates by electrospray ionization time-of-flight mass spectrometry, J. Mass. Spectrom. 46 (5) (2011) 478-488. DOI: 10.1002/jms.1914.

[150] P. Perez-Ortega, F.J. Lara-Ortega, J.F. Garcia-Reyes, B. Gilbert-Lopez, M. Trojanowicz, A. Molina-Diaz, A feasibility study of UHPLC-HRMS accurate-mass screening methods for multiclass testing of organic contaminants in food, Talanta. 160 (2016) 704-712. DOI: 10.1016/j.talanta.2016.08.002.

[151] A.W. Bristow, W.F. Nichols, K.S. Webb, B. Conway, Evaluation of protocols for reproducible electrospray in-source collisionally induced dissociation on various liquid chromatography/mass spectrometry instruments and the development of spectral libraries, Rapid. Commun. Mass Spectrom. 16 (24) (2002) 2374-2386. DOI: $10.1002 / \mathrm{rcm} .843$.

[152] J.T. Davidson, Z.J. Sasiene, G.P. Jackson, Fragmentation pathways of odd- and evenelectron $N$-alkylated synthetic cathinones, Int. J. Mass Spectrom. Accepted for publication (2020). DOI: 10.1016/j.ijms.2020.116354.

[153] G.A. Olah, R.J. Spear, D.A. Forsyth, Rearrangement of Ethylenebenzenium Ions to aPhenylethyl (Styryl) Cations. Determination of the Relative Energies of the a-Bridged Ethylenebenzenium Ion, the Open-Chain 2-Phenylethyl Cation, and the a-Styryl Cation, J. Am. Chem. Soc. 98 (20) (1976) 6284-6289.

[154] K.B. Palmquist, M. Swortwood, Quantification of Furanyl Fentanyl and its Metabolites in Human and Rat Plasma using LC-MS/MS, J. Anal. Toxicol. Accepted Manuscript (2020). DOI: 10.1093/jat/bkaa013.

[155] I. Zancanaro, R.P. Limberger, P.O. Bohel, M.K. dos Santos, R.B. De Boni, F. Pechansky, E.D. Caldas, Prescription and illicit psychoactive drugs in oral fluid--LC-MS/MS method development and analysis of samples from Brazilian drivers, Forensic Sci. Int. 223 (1-3) (2012) 208-216. DOI: 10.1016/j.forsciint.2012.08.048.

[156] A.D. Lesiak, R.A. Musah, R.B. Cody, M.A. Domin, A.J. Dane, J.R. Shepard, Direct analysis in real time mass spectrometry (DART-MS) of "bath salt" cathinone drug mixtures, Analyst. 138 (12) (2013) 3424-32. DOI: 10.1039/c3an00360d.

[157] A.H. Grange, G.W. Sovocool, Automated determination of precursor ion, product ion, and neutral loss compositions and deconvolution of composite mass spectra using ion correlation based on exact masses and relative isotopic abundances, Rapid Commun. Mass Spectrom. 22 (15) (2008) 2375-2390. DOI: 10.1002/rcm.3619. 
[158] H. Brown, B. Oktem, A. Windom, V. Doroshenko, K. Evans-Nguyen, Direct Analysis in Real Time (DART) and a portable mass spectrometer for rapid identification of common and designer drugs on-site, Forens. Chem. 1 (2016) 66-73. DOI: 10.1016/j.forc.2016.07.002. 


\section{Curriculum vitae}

\section{Education:}

\section{J. Tyler Davidson}

ORCID ID: 0000-0001-9932-8273

- West Virginia University, 1600 University Ave., Morgantown, WV (2015-Present)

- Doctor of Philosophy in Forensic Science (2017- Expected May 16, 2020)

- Dr. Glen P. Jackson

- Structural Characterization of Emerging Synthetic Drugs

- Master of Science in Forensic and Investigative Science (2015-2017) FEPAC Accredited

- Dr. Glen P. Jackson

- Analysis of 2,5-Dimethoxy-N-(N-methoxybenzyl)phenethylamines (NBOMe) Isomers Using Traditional and Fast Gas Chromatography-Mass Spectrometry

- Shippensburg University, 1871 Old Main Drive, Shippensburg, PA (2011-2015)

- Bachelor of Science in Chemistry ACS Certified

- Dr. Daniel P. Predecki and Dr. John N. Richardson (Fall 2014-Spring 2015)

- Qualitative and Quantitative Analysis of Fluorine Containing Synthetic Cannabinoids

\section{Awards, Honors, Scholarships:}

- American Academy of Forensic Sciences Emerging Forensic Scientists Award (2020)

- WVU Foundation Distinguished Doctoral Scholarship (Spring 2020)

- WVU Eberly College Outstanding Graduate Teaching Assistant (2018-2019)

- Blaney Graduate Fellowship WVU FIS Department (2016-2019)

- Society of Analytical Chemists of Pittsburgh Award Shippensburg University (2014-2015)

- Association of Pennsylvania State College \& University Faculties (APSCUF) Chemistry Student of the Year Award Shippensburg University (2012-2013)

- Board of Governors Scholarship Shippensburg University (2011-2015)

\section{Professional Memberships and Positions of Responsibility:}

- American Academy of Forensic Sciences (AAFS) (2020-Present)

- American Society for Mass Spectrometry (ASMS) (2018-Present)

- Ad Hoc Reviewer for Journal of Forensic Chemistry (2017-Present)

- American Chemical Society (ACS) (2015-Present) 


\section{Publications:}

10) J. T. Davidson, Z. J. Sasiene, G. P. Jackson, "Comparison of in-source collision-induced dissociation and beam-type collision-induced dissociation of synthetic cathinones and fentanyl analogs using a high-resolution quadrupole time-of-flight (Q-TOF) mass spectrometer," Rapid Commun. Mass Spectrom., 2020: Submitted.

9) J. T. Davidson, Z. J. Sasiene, G.P. Jackson, "Fragmentation pathways of odd- and evenelectron $N$-alkylated synthetic cathinones," Int. J. Mass Spectrom., 2020: Accepted for publication.

8) J. T. Davidson, Z. J. Sasiene, Y. Abiedalla, J. DeRuiter, C. R. Clark, G. P. Jackson, "Fragmentation pathways of $\alpha$-pyrrolidinophenone derivative synthetic cathinones and their application to the identification of novel synthetic cathinone derivatives," Int. J. Mass Spectrom., 2020: Accepted for publication; 10.1016/j.ijms.2020.116343.

7) J. T. Davidson, E. L. Piacentino, Z. J. Sasiene, Y. Abiedalla, J. DeRuiter, C. R. Clark, G. Berden, J. Oomens, V. Ryzhov, G. P. Jackson, "Identification of novel fragmentation pathways and fragment ion structures in the tandem mass spectra of protonated synthetic cathinones," Forens. Chem., 2020: Accepted for publication; 10.1016/j.forc.2020.100245.

6) J. T. Davidson, Z. J. Sasiene, G. P. Jackson, "The influence of chemical modifications on the fragmentation behavior of fentanyl and fentanyl-related compounds in electrospray ionization tandem mass spectrometry," Drug Test. Anal., 2020: Accepted for publication; 10.1002/dta.2794.

5) J. T. Davidson, Z. J. Sasiene, G. P. Jackson, "The characterization of isobaric product ions of fentanyl using multi-stage mass spectrometry, high-resolution mass spectrometry and isotopic labeling," Drug Test. Anal., 2020(12): 496-503. DOI: 10.1002/dta.2758.

4) I. C. Willis, Z. Fan, J. T. Davidson, G. P. Jackson, "Weathering of ignitable liquids at elevated temperatures: a thermodynamic model, based on laws of ideal solutions, to predict weathering in structure fires," Forens. Chem., 2020(18): 100215. DOI: 10.1016/j.forc.2020.100215.

3) J. T. Davidson, G. P. Jackson, "The differentiation of 2,5-dimethoxy-N-(Nmethoxybenzyl)phenethylamine (NBOMe) isomers using $\mathrm{GC}$ retention indices and multivariate analysis of ion abundances in electron ionization mass spectra," Forens. Chem., 2019(14): 100160. DOI: 10.1016/j.forc.2019.100160.

2) J. T. Davidson, B. J. Lum, G. Nano, G. P. Jackson, "Comparison of measured and recommended acceptance criteria for the analysis of seized drugs using Gas 
Chromatography-Mass Spectrometry (GC-MS)," Forens. Chem., 2018(10): 15-26. DOI: 10.1016/j.forc.2018.07.001.

1) M. K. dos Santos, E. Gleco, J. T. Davidson, G. P. Jackson, R. P. Limberger, L. E. Arroyo, "DART-MS/MS screening for the determination of 1,3-dimethylamylamine and undeclared stimulants in seized dietary supplements from Brazil," Forens. Chem., 2018(8): 134-145. DOI: 10.1016/j.forc.2018.03.005.

\section{Presentations:}

*Presenting author listed first.

26) G. P. Jackson, J. T. Davidson, Z. J. Sasiene, B. Lowe, Y. Abiedalla, C. R. Clark, E. L. Piacentino, V. Ryzhov, "Towards an Improved Understanding of the Mass Spectrometric Identification of Cathinones and Fentalogs," presented at PITTCON, Chicago, Illinois. March 2020. (Oral)

25) C. Wensel, I. C. Willis, J. T. Davidson, N. K. Eklund, A. L. Setser, V. McGuffin, R. W. Smith, G. P. Jackson, "Thermodynamic and Kinetic Predictions of the Evaporation Patterns of Ignitable Liquids at Elevated Temperatures," presented at PITTCON, Chicago, Illinois. March 2020. (Oral)

24) J. T. Davidson, Z. J. Sasiene, Y. Abiedalla, R. Clark, J. DeRuiter, G. P. Jackson, “On the Fragmentation Behavior of Fentanyl and Its Analogs in Electrospray Ionization-Tandem Mass Spectrometry (ESI-MS/MS)," presented at American Academy of Forensic Sciences $72^{\text {nd }}$ Annual Meeting, Anaheim, California. February 2020. (Oral)

23) S. A. Mehnert, B. D. Lowe, E. Ruiz, J. T. Davidson, G. P. Jackson, “A Regression-Based Algorithm to Maximize the Confidence in Mass Spectral Identifications," presented at American Academy of Forensic Sciences $72^{\text {nd }}$ Annual Meeting, Anaheim, California. February 2020. (Oral)

22) C. Wensel, I. Willis, Z. Fan, J. T. Davidson, G. P. Jackson, “The Effects of Elevated Temperature and Substrates on the Weathering of Ignitable Liquids," presented at American Academy of Forensic Sciences $72^{\text {nd }}$ Annual Meeting, Anaheim, California. February 2020. (Oral)

21) G. P. Jackson, S. A. Mehnert, B. D. Lowe, E. Ruiz, J. T. Davidson, “A Regression-Based Algorithm to Maximize the Confidence in Mass Spectral Identifications," presented at Eastern Analytical Symposium, Plainsboro, New Jersey. November 2019. (Oral)

20) S. Mehnert, B. Lowe, E. Ruiz, J. T. Davidson, "Development of a Flexible Algorithm for Substance Identification Using Mass Spectrometry,” presented at Eastern Analytical Symposium, Plainsboro, New Jersey. November 2019. (Poster) 
19) G. P. Jackson, S. Mehnert, B. Lowe, J. T. Davidson, “A Regression-based Algorithm to Maximize the Confidence in Mass Spectral Identifications," presented at SciX Conference, Palm Springs, California. October 2019. (Oral)

18) G. P. Jackson, J. T. Davidson, Z. J. Sasiene, Y. Abiedalla, J. DeRuiter, R. Clark, "On the Mass Spectral Interpretation of Cathinones and Fentanyl Analogs," presented at SciX Conference, Palm Springs, California. October 2019. (Oral)

17) J. T. Davidson, Z. J. Sasiene, Y. Abiedalla, C. R. Clark, G. P. Jackson, "Fragmentation Pathways of $\alpha$-Pyrrolidinophenone Derivative Synthetic Cathinones," presented at American Society for Mass Spectrometry $67^{\text {th }}$ Annual Meeting, Atlanta, Georgia. June 2019. (Poster)

16) G. P. Jackson, J. T. Davidson, Z. J. Sasiene, Y. Abiedalla, C. R. Clark, “On the Tandem Mass Spectrometry of Cathinones and Mass Spectrometric Identification of Drugs," presented at American Chemical Society $257^{\text {th }}$ National Meeting, Orlando, Florida. March 2019. (Oral)

15) G. P. Jackson, S. A. Mehnert, B. D. Lowe, J. T. Davidson, "On the Tandem Mass Spectrometry of Cathinones and Mass Spectrometric Identification of Drugs," presented at American Chemical Society 257 ${ }^{\text {th }}$ National Meeting, Orlando, Florida. March 2019. (Poster)

14) G. P. Jackson, S. A. Mehnert, B. D. Lowe, J. T. Davidson, "Development of a Flexible Algorithm for Substance Identification Using Mass Spectrometry," presented at PITTCON, Philadelphia, Pennsylvania. March 2019. (Poster)

13) I. C. Willis, Z. Fan, J. T. Davidson, G. P. Jackson, “The Influence of Elevated Temperatures on the Weathering of Ignitable Liquids," presented at PITTCON, Philadelphia, Pennsylvania. March 2019. (Poster)

12) J. T. Davidson, Z. J. Sasiene, Y. F. Abiedalla, C. R. Clark, G. P. Jackson, "The Identification of a Novel Fragmentation Pathway of Synthetic Cathinones," presented at American Academy of Forensic Sciences $71^{\text {st }}$ Annual Meeting, Baltimore, Maryland. February 2019. (Oral)

11) S. A. Mehnert, B. D. Lowe, J. T. Davidson, G. P. Jackson, “The Development of a Flexible Algorithm for Substance Identification Using Mass Spectrometry," presented at American Academy of Forensic Sciences $71^{\text {st }}$ Annual Meeting, Baltimore, Maryland. February 2019. (Oral) 
10) S. E. Chaffman, T. Williams, J. T. Miller, J. T. Davidson, G. P. Jackson, "Identification of an Ultraviolet (UV)-Induced Promethazine Dimer," presented at American Academy of Forensic Sciences $71^{\text {st }}$ Annual Meeting, Baltimore, Maryland. February 2019. (Poster)

9) G. P. Jackson, J. T. Davidson, Z. J. Sasiene, Y. Abiedalla, C. R. Clark, "On the Mass Spectrometric Identification of Synthetic Cathinones," presented at Australia and New Zealand Forensic Science Society, Perth, Australia. September 2018. (Oral)

8) S. A. Mehnert, B. D. Lowe, J. T. Davidson, G. P. Jackson, "Development of a Flexible Algorithm for Substance Identification Using Mass Spectrometry," presented at West Virginia University, Morgantown, West Virginia. July 2018. (Poster)

7) B. D. Lowe, S. A. Mehnert, J. T. Davidson, G. P. Jackson, "Development of a More Selective Mass Spectral Identification Algorithm," presented at West Virginia University, Morgantown, West Virginia. July 2018. (Poster)

6) G. P. Jackson, J. T. Davidson "MS Comparator: Ultra-Precise Spectral Comparisons," presented at the Forensic and Homeland Security Workshop of the 66th ASMS Conference on Mass Spectrometry and Allied Topics, San Diego, California. June 2018. (Oral)

5) J. T. Davidson, Z. J. Sasiene, Y. Abiedalla, C. R. Clark, G. P. Jackson, "Identification of a Novel Fragmentation Pathway of Synthetic Cathinones," presented at American Society for Mass Spectrometry $66^{\text {th }}$ Annual Meeting, San Diego, California. June 2018. (Poster)

4) H. Santos, J. T. Davidson, J. Cox, G. P. Jackson, W. Romão, L. E. Arroyo, "Potential Applications to New Psychoactive Substances Identification in Oral Fluid and Damiana Leaf (Turnera Diffusa) by DART-MS/MS and LC-MS/MS," presented at American Society for Mass Spectrometry $66^{\text {th }}$ Annual Meeting, San Diego, California. June 2018. (Poster)

3) J. T. Davidson, B. J. Lum, G. Nano, G. P. Jackson, "Quantifying the Uncertainty of Measurement of Gas Chromatography-Mass Spectrometry (GC/MS) Acceptance Criteria," presented at American Academy of Forensic Sciences $70^{\text {th }}$ Annual Meeting, Seattle, Washington. February 2018. (Poster)

2) J. T. Davidson, G. P. Jackson, "The Analysis of 2,5-Dimethoxy-N-(N-methoxybenzyl)phenethylamine (NBOMe) Isomers Using Traditional and Fast Gas Chromatography/Mass Spectrometry (GC/MS)," presented at American Academy of Forensic Sciences $69^{\text {th }}$ Annual Meeting, New Orleans, Louisiana. February 2017. (Poster)

1) J. T. Davidson, D. P. Predecki, J. N. Richardson, "Qualitative and Quantitative Analysis of Fluorine Containing Synthetic Cannabinoids," presented at American Chemical Society $249^{\text {th }}$ National Meeting, Denver, Colorado. March 2015. (Poster) 